Florida International University FIU Digital Commons

6-14-2018

\title{
Augmented Terrain-Based Navigation to Enable Persistent Autonomy for Underwater Vehicles in GPS-Denied Environments
}

Gregory M. Reis

Florida International University, greis003@fiu.edu

DOI: 10.25148 /etd.FIDC006900

Follow this and additional works at: https://digitalcommons.fiu.edu/etd

Part of the Artificial Intelligence and Robotics Commons

\section{Recommended Citation}

Reis, Gregory M., "Augmented Terrain-Based Navigation to Enable Persistent Autonomy for Underwater Vehicles in GPS-Denied Environments" (2018). FIU Electronic Theses and Dissertations. 3736.

https://digitalcommons.fiu.edu/etd/3736 


\section{FLORIDA INTERNATIONAL UNIVERSITY}

Miami, Florida

AUGMENTED TERRAIN-BASED NAVIGATION TO ENABLE PERSISTENT AUTONOMY FOR UNDERWATER VEHICLES IN GPS-DENIED ENVIRONMENTS

A dissertation submitted in partial fulfillment of the requirements for the degree of DOCTOR OF PHILOSOPHY in COMPUTER SCIENCE

by

Gregory Murad Reis 
To: Dean John L. Volakis

College of Engineering and Computing

This dissertation, written by Gregory Murad Reis, and entitled Augmented Terrain-Based Navigation to Enable Persistent Autonomy for Underwater Vehicles in GPS-Denied Environments, having been approved in respect to style and intellectual content, is referred to you for judgment.

We have read this dissertation and recommend that it be approved.

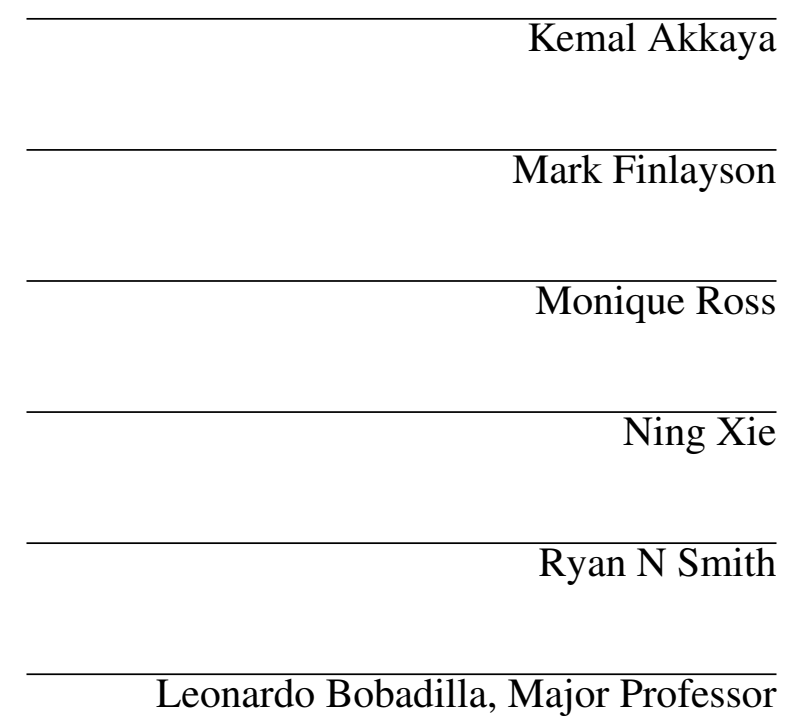

Date of Defense: June 14, 2018

The dissertation of Gregory Murad Reis is approved.

Dean John L. Volakis College of Engineering and Computing

Andres G. Gil

Vice President for Research and Economic Development and Dean of the University Graduate School

Florida International University, 2018 
(C) Copyright 2018 by Gregory Murad Reis

All rights reserved. 


\section{DEDICATION}

This is dedicated to my father Paulo, my mother Dulce, my sister Mariane, my nieces

Rachel and Rebeca, my grandparents Alberto and Aurea and my godmothers Clelia and Tania. 


\section{ACKNOWLEDGMENTS}

I would to thank God for the uncountable blessings in my life, especially in this Ph.D. journey. This dissertation would never have been possible without the assistance and support of a lot of amazing people.

I would have never been able to accomplish anything in life without the unconditional love and support of my parents, Dulce and Paulo. Their encouragement, understanding and patience made me who I am today.

I offer sincere thanks to my advisor, Dr. Leonardo Bobadilla, who guided me from the beginning, mentored me as a professional, taught me and trusted me this work. $\mathrm{He}$ became my friend, a person by whom I am inspired. His continuous encouragement, support, and technical suggestions contributed to this dissertation. He made me interested in doing research on robotics and gave me the chance to teach robotics for over two years. I would like to express my sincere gratitude to Dr. Ryan Smith, for being my co-advisor, mentor and opened the doors of the field of underwater robotics so I could have this research done. I learned a lot from several insightful discussions with him and I am glad I had the chance to work close to him twice in Durango, CO. And special thanks to all my dissertation committee members: Dr. Kemal Akkaya, Dr. Mark Finlayson, Dr. Monique Ross and Dr. Ning Xie for their time and valuable feedback to improve my dissertation.

I shared my work environment with amazing Ph.D. students: Triana, Sebastian, Tauhidul, Hector, Mahbubur, among several other throughout these years. Sebastian. It was intellectually stimulating and enjoyable to work together with these smart people who provided me lots of constructive feedback about my research papers and presentations.

I am also thankful Ph.D. colleagues Deya Banisakher and Andrius Bubelis, who turned out to be my best friends and who I wish to continue being close friends for a long long 
time.

Finally, I would like to acknowledge the financial support of CAPES, Brazilian government, Laspau and Florida International University Graduate School. This dissertation was also supported in part by the U.S. Department of Homeland Security under Grant Award Number 2017-ST-062000002, by the Office of Naval Research Award Number N000141612634 and by the National Science Foundation MRI Award Number: 1531322. 


\author{
ABSTRACT OF THE DISSERTATION \\ AUGMENTED TERRAIN-BASED NAVIGATION TO ENABLE PERSISTENT \\ AUTONOMY FOR UNDERWATER VEHICLES IN GPS-DENIED ENVIRONMENTS \\ by \\ Gregory Murad Reis \\ Florida International University, 2018 \\ Miami, Florida \\ Professor Leonardo Bobadilla, Major Professor
}

Aquatic robots, such as Autonomous Underwater Vehicles (AUVs), play a major role in the study of ocean processes that require long-term sampling efforts and commonly perform navigation via dead-reckoning using an accelerometer, a magnetometer, a compass, an IMU and a depth sensor for feedback. However, these instruments are subjected to large drift, leading to unbounded uncertainty in location. Moreover, the spatio-temporal dynamics of the ocean environment, coupled with limited communication capabilities, make navigation and localization difficult, especially in coastal regions where the majority of interesting phenomena occur. To add to this, the interesting features are themselves spatio-temporally dynamic, and effective sampling requires a good understanding of vehicle localization relative to the sampled feature.

Therefore, our work is motivated by the desire to enable intelligent data collection of complex dynamics and processes that occur in coastal ocean environments to further our understanding and prediction capabilities. The study originated from the need to localize and navigate aquatic robots in a GPS-denied environment and examine the role of the spatio-temporal dynamics of the ocean into the localization and navigation processes. The methods and techniques needed range from the data collection to the localization and navigation algorithms used on-board of the aquatic vehicles. The focus of this work is to develop algorithms for localization and navigation of AUVs in GPS-denied environ- 
ments. We developed an Augmented terrain-based framework that incorporates physical science data, i.e., temperature, salinity, $\mathrm{pH}$, etc., to enhance the topographic map that the vehicle uses to navigate. In this navigation scheme, the bathymetric data are combined with the physical science data to enrich the uniqueness of the underlying terrain map and increase the accuracy of underwater localization. Another technique developed in this work addresses the problem of tracking an underwater vehicle when the GPS signal suddenly becomes unavailable. The methods include the whitening of the data to reveal the true statistical distance between datapoints and also incorporates physical science data to enhance the topographic map.

Simulations were performed at Lake Nighthorse, Colorado, USA, between April 25th and May 2nd 2018 and at Big Fisherman's Cove, Santa Catalina Island, California, USA, on July 13th and July 14th 2016. Different missions were executed on different environments (snow, rain and the presence of plumes).

Results showed that these two methodologies for localization and tracking work for reference maps that had been recorded within a week and the accuracy on the average error in localization can be compared to the errors found when using GPS if the time in which the observations were taken are the same period of the day (morning, afternoon or night). The whitening of the data had positive results when compared to localizing without whitening. 


\section{TABLE OF CONTENTS}

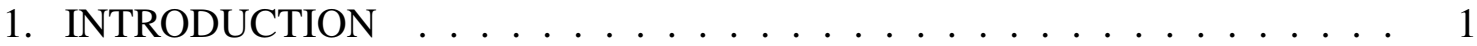

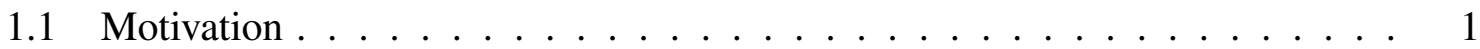

1.2 Mobile Robots . . . . . . . . . . . . . . . . . . . . . . . 3

1.3 Localization of Autonomous Underwater Vehicles . . . . . . . . . . . . . 6

1.3.1 Solutions to the Problem of Localization . . . . . . . . . . . . . . . 7

1.4 Organization of the Dissertation $\ldots \ldots \ldots \ldots$

2. DATA COLLECTION . . . . . . . . . . . . . . . . . . 13

2.1 Water Parameters . . . . . . . . . . . . . . . . . . . . . . 13

2.1 .1 Temperature . . . . . . . . . . . . . . . . . . . . . . 13

2.1 .2 Salinity . . . . . . . . . . . . . . . . . . . . . . . 14

2.1 .3 Dissolved Oxygen . . . . . . . . . . . . . . . . . . . . 14

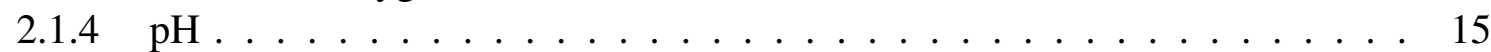

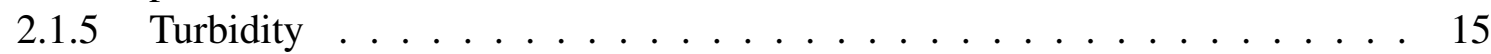

2.1 .6 Chlorophyll . . . . . . . . . . . . . . . . . . . . 16

2.2 The autonomous underwater vehicles (AUVs) . . . . . . . . . . . . . 17

$2.2 .1 \quad$ Santa Catalina Island, CA, USA . . . . . . . . . . . . . . . . . 18

2.2 .2 Lake Nighthorse, CO, USA . . . . . . . . . . . . . . . . . 20

2.2 .3 Monterey Bay, CA . . . . . . . . . . . . . . . . . 23

3. INFORMATIVE PATH PLANNING . . . . . . . . . . . . . . . . . . 24

3.1 Overview . . . . . . . . . . . . . . . . . . . . . 24

3.2 Trajectory Design with Predictive Ocean Models . . . . . . . . . . . . . 27

3.3 Dealing with Uncertainty . . . . . . . . . . . . . . . . . . . 28

3.4 Spatial Interpolation . . . . . . . . . . . . . . . . . . . . . . . . . . . . . . . . . . 29

$3.4 .1 \quad$ Spline . . . . . . . . . . . . . . . . . . . . . . . . . . . . . . . . . . . . 29

3.4.2 Inverse Distance Weighting (IDW) . . . . . . . . . . . . . . . . . 30

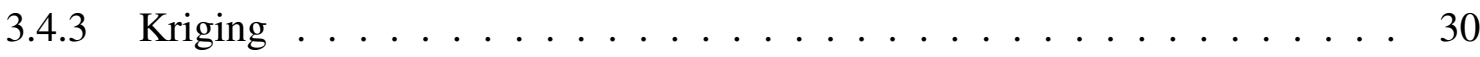

3.5 Informative Path Planning for Optimal Sampling . . . . . . . . . . . . 42

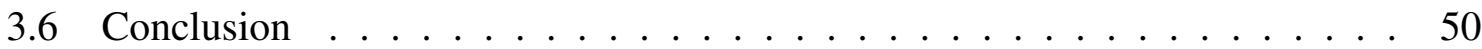

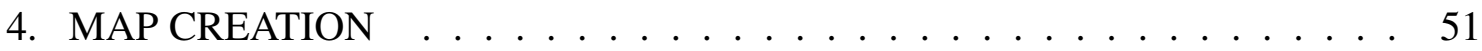

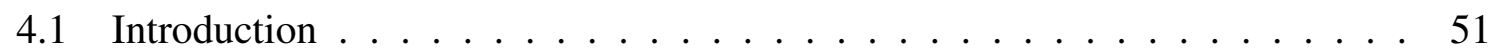

4.2 Related Work . . . . . . . . . . . . . . . . . . . . . . 51

$4.2 .1 \quad$ Ecological Niche Models . . . . . . . . . . . . . . . . . . . . . 52

4.2.2 Reference Maps in Localization . . . . . . . . . . . . . . . . . . . . . 54

4.3 Augmented Terrain-Based Navigation Framework . . . . . . . . . . . . . 55

4.3.1 Overview . . . . . . . . . . . . . . . . . . . . 55

4.3.2 Global Correlation Score . . . . . . . . . . . . . . . . . . . . 56

4.3.3 Weighting Scheme . . . . . . . . . . . . . . . . . . 57 
4.3.4 Spatial Autocorrelation . . . . . . . . . . . . . . . . . . . . . 61

4.4 Preliminary Results . . . . . . . . . . . . . . . . . . . . . . . . . 67

4.4.1 Sampling in the Simplex and Global Autocorrelation Score . . . . . . . . 67

4.4.2 Dirichlet Distribution and Global Autocorrelation Score . . . . . . . . . 70

4.4.3 Discussion on Spatial and Temporal Terrain Map Analysis . . . . . . . . 72

4.5 Conclusion . . . . . . . . . . . . . . . . 76

5. GLOBAL LOCALIZATION . . . . . . . . . . . . . . . . . . 80

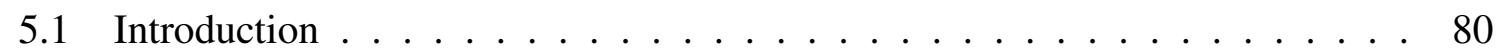

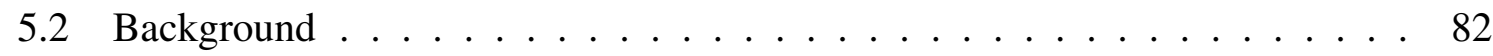

$5.2 .1 \quad$ Localization . . . . . . . . . . . . . . . . . . . . . . . 82

5.2 .2 Inertial Navigation . . . . . . . . . . . . . . . . . . . . . . . . 84

5.2 .3 Acoustic Navigation . . . . . . . . . . . . . . . . . . . 85

5.2 .4 Geophysical Navigation $\ldots \ldots \ldots$. . . . . . . . . . . . 86

5.2 .5 Improving TBN . . . . . . . . . . . . . . . . . . . . 88

5.3 METHODS . . . . . . . . . . . . . . . . . . . . . . . . . . . . 89

5.4 Global Localization Within the Terrain Map . . . . . . . . . . . . . . . . . 89

$5.4 .1 \quad$ Localization Results . . . . . . . . . . . . . . . . . . . . . . . . . 91

5.5 CONCLUSIONS AND FUTURE WORK . . . . . . . . . . . . . 95

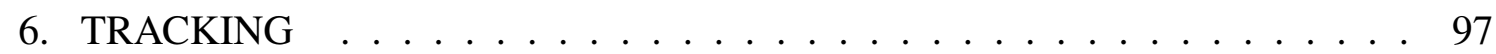

6.1 Overview . . . . . . . . . . . . . . . . . . . . . 97

6.2 Methodology . . . . . . . . . . . . . . . . . . . . . . 98

6.3 Related Work . . . . . . . . . . . . . . . . . . . . . . . . . . 100

6.3.1 Robot Localization and Tracking . . . . . . . . . . . . . . . . . . 100

6.3.2 Terrain-Based Navigation . . . . . . . . . . . . . . . . . . . . 101

6.3 .3 Data Whitening . . . . . . . . . . . . . . . . . . . 102

6.4 Preliminaries . . . . . . . . . . . . . . . . . . . . . . 102

6.4 .1 Model Definition . . . . . . . . . . . . . . . . . . . . . . . . 102

6.4 .2 Problem Formulation . . . . . . . . . . . . . . . . . . . 103

6.5 Methods . . . . . . . . . . . . . . . . . . . . . . 106

6.5 .1 Data Acquisition . . . . . . . . . . . . . . . . . 106

6.5 .2 Data Preprocessing . . . . . . . . . . . . . . . . . . . . . 106

6.5.3 Decorrelation Process . . . . . . . . . . . . . . . . . . 106

6.5 .4 ZCA Mahalanobis . . . . . . . . . . . . . . . . . . . . . 108

6.5 .5 Localization Algorithm . . . . . . . . . . . . . . . . . . . . . . . . 114

6.5.6 Results in Santa Catalina Island, CA . . . . . . . . . . . . . . . 115

6.5.7 Results at the Lake Nighthorse, CO . . . . . . . . . . . . . . . . . . . . 119

6.5.8 Conclusion and Future Work . . . . . . . . . . . . . . . . . . 133

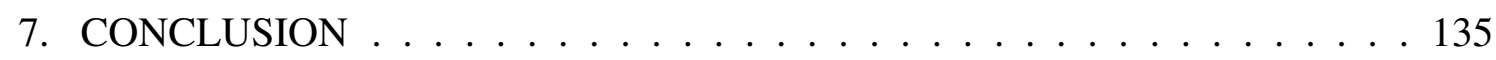

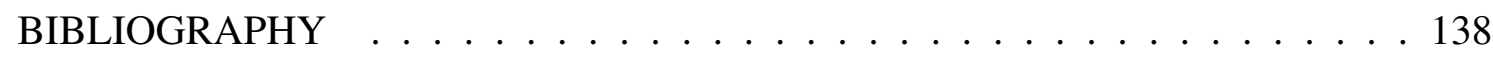




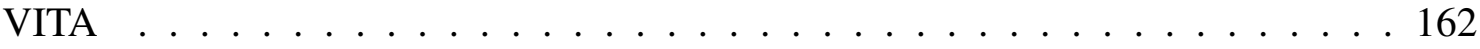




\section{LIST OF TABLES}

TABLE

PAGE

2.1 Descriptive statistics for the dataset collected during a mission at the Big Fisherman's Cove, Santa Catalina Island, CA, USA, on July 13th 2016 . 20

2.2 Descriptive statistics for the dataset collected during a mission at the Big Fisherman's Cove, Santa Catalina Island, CA, USA, on July 14th 2016. . 20

2.3 Descriptive statistics for the dataset collected during a mission at the Lake Nighthorse, CO, USA, on April 25th 2018, in the afternoon. . . . . . . . 22

2.4 Descriptive statistics for the dataset collected during a mission at the Lake Nighthorse, CO, USA, on May 2nd 2018, in the morning. . . . . . . . . 22

2.5 Descriptive statistics for the dataset collected during a mission at the Lake Nighthorse, CO, USA, on May 2nd 2018, in the afternoon. . . . . . . . 23

4.1 Global correlation values for different combination of parameters. . . . . . . 70

5.1 Minimum, maximum, mean and standard deviation of the water parameters considered for the Santa Catalina Island deployment on the first day. . . . 92

5.2 Minimum, maximum, mean and standard deviation of the water parameters considered for the Santa Catalina Island deployment on the second day. . 92

5.3 Weights for the water parameters for two different missions in two different days in Santa Catalina Island, CA, USA . . . . . . . . . . . . . . . 94

6.1 Covariance matrix of data set with three parameters . . . . . . . . . 113

6.2 Interpretation of the boxplots for multiple missions . . . . . . . . . . . 134 


\section{LIST OF FIGURES}

FIGURE

PAGE

1.1 Examples of mobile robots: (a) The Google's Waymo self-driving car [Inc18];

(b) DJI Matrice 210 for precision agriculture [Haw18]; (c) Boston Dynamics's humanoid robot Atlas [Dyn18a]; (d) Boston Dynamics's doglike robot SpotMini [Dyn18b]; (e) NASA's Curiosity rover for Mars exploration [oTTJPL18a] and [Ima18]; (f) The NASA's Mars Helicopter [oTTJPL18b]; (g) The YSI Ecomapper autonomous underwater vehicle (AUV) [Incb]; (h) The Clearpath's Heron autonomous surface vessel (ASV) [Rob18]; (i) AQUA2 Autonomous Underwater Vehicle [OAM16].

1.2 Organization of this dissertation with arrows indicating dependencies. . . . . 11

2.1 (a) Diagram of YSI 6035 chlorophyll crobe and (b) Diagram of EXO total algae (Chlorophyll + Blue-green Algae) probe. Images obtained from the Tech Note on The Basics of Chlorophyll Measurement in [Inc14b].

2.2 The YSI Ecomapper underwater vehicle used in the data collection of this work. . . . . . . . . . . . . . . .

2.3 Aerial Image of the Big Fisherman's Cove, Santa Catalina Island, CA, USA (a) and the Lake Nighthorse, CO, USA (b) . . . . . . . . . . . . .

3.1 Semivariograms of the depth $(\mathrm{m})$ parameter generated in the datasets collected in Santa Catalina Island on July 13th 2016. . . . . . . . . . . . . . 32

3.2 Semivariograms of the depth $(\mathrm{m})$ parameter generated in the datasets collected in Santa Catalina Island on July 14th 2016. . . . . . . . . . . 33

3.3 Semivariograms of the temperature $\left({ }^{\circ} \mathrm{C}\right)$ parameter generated in the datasets collected in Santa Catalina Island on July 13th 2016 . . . . . . . . . 33

3.4 Semivariograms of the temperature $\left({ }^{\circ} \mathrm{C}\right)$ parameter generated in the datasets collected in Santa Catalina Island on July 14th 2016.

3.5 Semivariograms of the salinity (ppt) parameter generated in the datasets collected in Santa Catalina Island on July 13th 2016. . . . . . . . . . . . . . 34

3.6 Semivariograms of the salinity (ppt) parameter generated in the datasets collected in Santa Catalina Island on July 14th 2016. . . . . . . . . . . . 34

3.7 Semivariograms of the $\mathrm{pH}$ parameter generated in the datasets collected in Santa Catalina Island on July 13th 2016. . . . . . . . . . . . . . 34

3.8 Semivariograms of the $\mathrm{pH}$ parameter generated in the datasets collected in Santa Catalina Island on July 14th 2016. . . . . . . . . . . . . . . 3

3.9 Semivariograms of the turbidity (NTU) parameter generated in the datasets collected in Santa Catalina Island on July 13th 2016. . . . . . . . . . . . 35 
3.10 Semivariograms of the turbidity (NTU) parameter generated in the datasets collected in Santa Catalina Island on July 14th 2016. . . . . . . . . . . .

3.11 Semivariograms of the dissolved oxygen $(\mathrm{mg} / \mathrm{L})$ parameter generated in the datasets collected in Santa Catalina Island on July 13th 2016. . . . . . . 36

3.12 Semivariograms of the dissolved oxygen $(\mathrm{mg} / \mathrm{L})$ parameter generated in the datasets collected in Santa Catalina Island on July 14th 2016. . . . . . . 36

3.13 Semivariograms of the depth $(\mathrm{m})$ parameter generated in the datasets collected at Lake Nighthorse on May 2nd 2018. . . . . . . . . . . . . . . . 36

3.14 Semivariograms of the temperature $\left({ }^{\circ} \mathrm{C}\right)$ parameter generated in the datasets collected at Lake Nighthorse on May 2nd 2018. . . . . . . . . . . . . . .

3.15 Semivariograms of the salinity (ppt) parameter generated in the datasets collected at Lake Nighthorse on May 2nd 2018. . . . . . . . . . . . . . . 3

3.16 Semivariograms of the $\mathrm{pH}$ parameter generated in the datasets collected at Lake Nighthorse on May 2nd 2018. . . . . . . . . . . . . . . . . . .

3.17 Semivariograms of the turbidity (NTU) parameter generated in the datasets collected at Lake Nighthorse on May 2nd 2018. . . . . . . . . . . . . . .

3.18 Semivariograms of the dissolved oxygen $(\mathrm{mg} / \mathrm{L})$ parameter generated in the datasets collected at Lake Nighthorse on May 2nd 2018. . . . . . . . . .

3.19 Predictions of values for the water parameters depth (a), temperature (b), salinity (c), pH (d), turbidity (e) and dissolved oxygen (f) generated through Kriging in the datasets collected in Santa Catalina Island on July

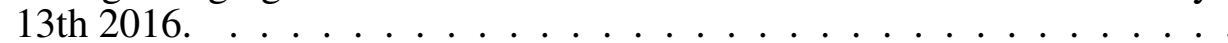

3.20 Predictions of values for the water parameters depth (a), temperature (b), salinity (c), pH (d), turbidity (e) and dissolved oxygen (f) generated through Kriging in the datasets collected in Santa Catalina Island on July 14 th 2016

3.21 Predictions of values for the water parameters depth (a), temperature (b), salinity (c), pH (d), turbidity (e) and dissolved oxygen (f) generated through Kriging in the datasets collected at Lake Nighthorse on May

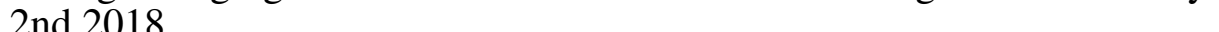

3.22 Errors in predictions for the water parameters depth (a), temperature (b), salinity (c), pH (d), turbidity (e) and dissolved oxygen (f) generated through Kriging and calculated using the square root of mean-squareerror for the datasets collected in Santa Catalina Island on July 14th 2016. 
3.23 Errors in predictions for the water parameters depth (a), temperature (b), salinity (c), $\mathrm{pH}(\mathrm{d})$, turbidity (e) and concentration of dissolved oxygen (f) generated through Kriging interpolation and calculated using the square root of mean-square-error for the datasets collected during a mission at Lake Nighthorse, Durango, Colorado, USA, on May 2nd 2018. . .

3.24 Simulation results of the long-term deployment policy for the informative sampling in (a) Santa Catalina Island, CA, USA for just bathymetry; (b) Santa Catalina Island, CA, USA for the combination of water parameters and bathymetry on the first day of deployment; (c) Santa Catalina Island, CA, USA for the combination of water parameters and bathymetry on the second day of deployment; (d) Lake Nighthorse, CO, USA for the combination of water parameters and bathymetry. . . . . . . . .

4.1 Dissimilarity measure based on for a random field. . . . . . . . . . . . . . 64

4.2 Semivariograms of the water parameters depth, temperature, salinity, $\mathrm{pH}$, turbidity and dissolved oxygen generated in the datasets collected in Santa Catalina Island on July 13th 2016 (left images) and July 14th 2016 (right images). . . . . . . . . . . . . . . .

4.3 For the combination of two science parameters with maximum variability:

(a) terrain map; (b) auto-correlogram. . . . . . . . . . . . . . . . 67

4.4 For the combination of the seven science parameters with minimum autocorrelation: (a) terrain map; (b) auto-correlogram. . . . . . . . . . . 68

4.5 Terrain map generated for the combination of science parameters and bathymetric information (a), auto-correlation for the bathymetric information (b) and auto-correlation for the combination of science parameters and bathymetric information (c). . . . . . . . . . . . .

4.6 Scalar field map at the Big Fisherman's Cove, Santa Catalina Island (a) and its auto-correlogram $(\mathrm{b}) . \ldots \ldots 71$

4.7 Scalar field map at the Lake Nighthorse, CO (a) and its auto-correlogram (b). 71

4.8 The individual parameter weights for two deployments on different days for both ocean (Top) and freshwater (Bottom) regions. . . . . . . . . 73

4.9 Individual parameter weights for four deployments covering both ocean and freshwater regions on different days. . . . . . . . . . . . . . 74

4.10 Individual parameter weights for two deployments covering different ocean regions at different times; Santa Catalina Island vs. Monterey Bay. . . . . 75

4.11 Final terrain maps computed for the following water parameters on July 13th 2016 in Santa Catalina Island, CA: depth (a), temperature (b), salinity (c), $\mathrm{pH}(\mathrm{d})$, turbidity (e) and dissolved oxygen (f) . . . . . . . . . 
4.12 Final terrain maps computed for the following water parameters on May 2nd 2018 at Lake Nighthorse, CO: depth (a), temperature (b), salinity (c), pH (d), turbidity (e) and dissolved oxygen (f) . . . . . . . . . . .

5.1 The underlying scalar field computed from the data gathered during a dense lawnmower path in the Big Fisherman's Cove off Santa Catalina Island, CA. . . .

5.2 The underlying scalar field computed from the data gathered during a dense lawnmower path in the Big Fisherman's Cove off Santa Catalina Island, CA. . . . . . . . . . . . . . . . . . .

5.3 Localization of a segment of a trajectory on the first day of deployment in the Big Fisherman's Cove off Santa Catalina Island, CA. The black segment represents the original trajectory and the red segment represents the approximate trajectory localized by the proposed methods. The colors in the plot varies according to the combination of water parameters and bathymetric information. The $\mathrm{x}$-axis is longitude and the $\mathrm{y}$-axis is latitude. $\ldots \ldots$

5.5 A bathymetric map (bottom) used for regular terrain-based navigation approaches and a combination of bathymetric information and water parameters (top) are placed together with the objective to compare the number of candidates for the same sensor reading. When just depth is analyzed, there are more candidates per observation than when the combination of bathymetric information and water data are combined. . . .

5.4 Localization of a trajectory from a mission on the first day of deployment at the Big Fisherman's Cove off Santa Catalina Island, CA. The orange segment represents the original trajectory, executed during the same survey (in blue) from which the reference map was created; the green segment represents the approximate trajectory localized by traditional terrain-based navigation systems; and the red trajectory represents the approximate trajectory localized by the augmented terrain-based navigation framework. The $\mathrm{x}$-axis is longitude and the $\mathrm{y}$-axis is latitude. . . . .

6.1 This image shows the region of data gathering during a dense lawnmower path in the Big Fisherman's Cove off Santa Catalina Island, CA, on the first (a) and second (b) days. . . . . . . . . . . . . . . . .

6.2 In (a), the correlated data is shown as blue dots and realize that the red and green crosses seem to have similar distances from the multivariate mean. The principal components are shown in (b) as black and red arrows (principal components are orthogonal to each other). In (c) data is projected onto the direction of the first principal component (rotation of the data). The data points are uncorrelated and normalized in this new space as shown in $(\mathrm{d}) \ldots \ldots \ldots \ldots \ldots$

6.3 a) Original centered data and (b) Eigenvectors of the covariance matrix. . . . 113 
6.4 Blue points represent the data-points during the first day of deployment; the green points represent the list of observations where GPS signal was not available; and the orange points represent the approximate localization of each one of these observations a) without the whitening process and b) using the whitening process. . . . . . . . . . . . 116

6.5 Comparison of the errors in GPS information using without whitening and with whitening methods for the first day of deployment. . . . . . . . 117

6.6 Blue points represent the data-points during the second day of deployment; the green points represent the list of observations where GPS signal was not available; and the orange points represent the approximate localization of each one of these observations a) without the whitening process and $b$ ) using the whitening process. . . . . . . . . . . . 117

6.7 Comparison of the errors in GPS information using without whitening and with whitening methods for the second day of deployment. . . . . . 118

6.8 The bathymetric map of the region of interest at the Lake Nighthorse, CO. . 120

6.9 The localized observations are shown in orange, while the original trajectory is shown in green. This trajectory was selected for test due to significant bathymetric relief. Blue points represent surveyed data for the first mission executed on April 25th 2018. In (a) the observations were localized on without the whitening process and in (b) the whitening process was

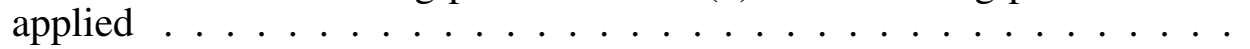

6.10 Comparison of the errors in GPS information without the whitening process (in blue) and with the whitening process (in orange) for the first mission executed on April 252018 . . . . . . . . . . . . . . . .

6.11 The localized observations are shown in orange, while the original trajectory is shown in green. This trajectory was selected for test due to significant bathymetric relief. Blue points represent surveyed data for the first mission executed on April 25th 2018. In (a) the observations were localized on without the whitening process and in (b) the whitening process was applied. . . . . . . . . . . . . . . 122

6.12 Comparison of the errors in GPS information without the whitening process (in blue) and with the whitening process (in orange) for the first mission executed on April 25 2018. . . . . . . . . . . . . . . . . . 12

6.13 The localized observations are shown in orange, while the original trajectory is shown in green. This trajectory was selected for test due to significant bathymetric relief. Blue points represent surveyed data for the first mission executed on April 25th 2018. In (a) the observations were localized on without the whitening process and in (b) the whitening process was applied with epsilon ball. . . . . . . . . . . . . . . . 123 
6.14 Comparison of the errors in GPS information without the whitening process (in blue) and with the whitening process (in orange) for the first mission executed on April 25th 2018.

6.15 The localized observations are shown in orange, while the original trajectory (extracted from a mission on May 2nd) is shown in green. This trajectory was selected for test due to significant bathymetric relief. Blue points represent surveyed data for the first mission executed on April 25th 2018. In (a) the observations were localized on without the whitening process and in (b) the whitening process was applied with epsilon ball. . . . . . . 124

6.16 Comparison of the errors in GPS information without the whitening process (in blue) and with the whitening process (in orange) . . . . . . . . 125

6.17 The localized observations are shown in orange, while the original trajectory (extracted from a mission on May 2nd) is shown in green. This trajectory was selected for being close to the shore. Blue points represent surveyed data for the first mission executed on April 25th 2018. In (a) the observations were localized on without the whitening process and in (b) the whitening process was applied. . . . . . . . . . 126

6.18 Comparison of the errors in GPS information without the whitening process (in blue) and with the whitening process (in orange). . . . . . . . . 126

6.19 The localized observations are shown in orange, while the original trajectory (extracted on the same day) is shown in green. This trajectory was selected for being close to the shore. Blue points represent surveyed data for the mission executed on May 2nd 2018. In (a) the observations were localized on without the whitening process and in (b) the whitening process was applied. . . . . . . . . . . . . . 127

6.20 Comparison of the errors in GPS information without the whitening process (in blue) and with the whitening process (in orange) . . . . . . . 128

6.21 The localized observations are shown in orange, while the original trajectory (extracted on the same day) is shown in green. This trajectory was selected for being close to the shore. Blue points represent surveyed data for the mission executed on May 2nd 2018. In (a) the observations were localized on without the whitening process and in (b) the whitening process was applied with epsilon ball. . . . . . . . . . . . . . . 128

6.22 Comparison of the errors in GPS information without the whitening process (in blue) and with the whitening process (in orange). . . . . . . . . . 129

6.23 The localized observations are shown in orange, while the original trajectory (extracted on the same day, but in the afternoon) is shown in green. This trajectory was selected for being close to the shore and with significant relief. Blue points represent surveyed data for the mission executed on May 2nd 2018 in the morning. In (a) the observations were localized on without the whitening process and in (b) the whitening process was applied with epsilon ball. 
6.24 Comparison of the errors in GPS information without the whitening process (in blue) and with the whitening process (in orange) . . . . . . . . 131

6.25 The localized observations are shown in orange, while the original trajectory (extracted on the same day, but in the afternoon) is shown in green. This trajectory was selected for being close to the shore and with significant relief. Blue points represent surveyed data for the mission executed on May 22018 in the morning. In (a) the observations were localized on without the whitening process and in (b) the whitening process was applied with epsilon ball. . . . . . . . . . . . . . . . . 132

6.26 Comparison of the errors in GPS information without the whitening process (in blue) and with the whitening process (in orange). . . . . . . . . 132 


\section{CHAPTER 1}

\section{INTRODUCTION}

\subsection{Motivation}

Significant study and research in the utility and implementation of autonomous underwater and surface vehicles (AUVs and ASVs) for ocean monitoring have been conducted in recent years. In most of these studies, the dynamics of physical phenomena, e.g., ocean fronts, occurence of harmful algae blooms, have been incorporated as parameters to aid in localization and navigation. To effectively observe and quantify these phenomena, it is necessary to measure several water properties and this must be done quickly enough to capture the spatial and temporal variability that occur in such a changing environment. Nevertheless, traditional oceanographic methods that use ships, buoys and floats, usually collect sparse measurements over periods of time that make the performance such tasks infeasible or difficult. To overcome this problem, it is required to employ an adaptivesampling, i.e., aquatic vehicles that can perform in situ feature recognition and that can respond to events with accurate localization; thus deepening our understanding of the dynamic oceanic processes. Therefore, an accurate localization of these aquatic vehicles is the main objective of this dissertation.

The ocean is a complex and dynamic environment given by an interaction between atmospheric, oceanographic, estuarine/riverine and landsea processes $\left[\mathrm{SCL}^{+} 10 \mathrm{a}\right]$. In order to understand and effectively study ocean processes, it is necessary to measure several water properties and analyze the spatial and temporal variability. To accomplish these tasks, long-term sampling efforts that can take from weeks to months need to match the duration of the respective oscillation patterns that one desires to study. Given the stochastic

environment and the large $\left(>50 \mathrm{~km}^{2}\right)$ spatial and temporal scales of significant processes and phenomena, sampling is sparse at best, and predictive models are necessary to aug- 
ment decision making to ensure that robots are in the right place and time for sampling. However, there is no single model that provides an informed view or representation of these or any other ocean feature that enables intelligent sampling in a principled manner. Thus, forecasting where a robot should sample in the immediate future is a challenging task. The use of persistent, autonomous underwater vehicles that have a similarly long deployment duration, and specifically, vehicles that can remain submerged for data collection for long periods of time, e.g. $\left[\mathrm{SKA}^{+} 07\right]-\left[\mathrm{CMG}^{+} 02 \mathrm{a}\right]$, are necessary. Our work is also motivated by the desire to enable intelligent data collection of complex dynamics and processes that occur in coastal ocean environments to further our understanding and prediction capabilities.

The autonomous underwater vehicles (AUVs) are being widely accepted and used in several civilian and military applications. The main reason for this is the fact that AUVs are safe, cost-effective and reliable when compared to systems that are manned and/or remotely controlled [HMH07]. One can think of a dangerous environment where personnel are exposed to threats such as in mine fields; thus, a robot can reduce this exposure and still provide flexibility and agility.

According to [MM17] and [HMH07], an autonomous underwater vehicle needs to perform well in three different tasks:

1. energy autonomy - the AUV needs to have a reliable power source that last during long deployment duration;

2. navigation autonomy - little to no error in estimating its position when in longperiod missions;

3. decision autonomy - make decision in unpredictable environments.

Therefore, it is expected that the level of autonomy of these aquatic robots is measure by the time it performs missions with little errors in estimation and as little human interven- 
tion as possible. Having truly autonomous robots is a challenging task that involves the robot to correctly estimate its position and attitude.

In this work, we seek are to overcome the theoretical and technical challenges of current localization techniques in GPS-denied environments and develop a framework for autonomous underwater vehicles (AUV) localization and sampling within dynamic ocean features. This work makes contributions to the areas of navigation, localization, prediction, surveillance, monitoring and mostly autonomy in marine robotics.

\subsection{Mobile Robots}

In recent years, robots have left the settings of controlled laboratories and began to execute tasks in real world scenarios by adapting to difference situations and making decisions. These robots are known as mobile robots and they are able to perceive the environment and apply techniques, e.g., mapping, navigation, localization, tracking and planning. The goals and challenges today is to perform these techniques more accurately, more reliably and more responsibly. These mobile robots are being employed in highly dynamic environments in the areas of manufacturing, agriculture, transportation, surveillance, healthcare, among others. Recent advances in technology, modeling and computational power and memory have allowed these robots to enter our society and impact our lives. The robotics industry together with government organizations are motivating the robotics researchers to investigate new techniques for more reliable robots and for decreasing the risks to people and the environment. The support comes in different ways, for example:

1. National Robotics Initiative - National Science Foundation (NSF), the National Aeronautics and Space Administration (NASA), the National Institutes of Health

(NIH), the U.S. Department of Agriculture (USDA), and the U.S. Department of Defense (DOD) partnered to advance in the development and utilization of robots 
in the United States of American that are able to work cooperatively with people (symbiotic relationships and innovation).

2. Waymo: Google self-driving car project - fully self-driving vehicles on public roads. These vehicles are able to use sensors to generate a detailed high-resolution 3D map of its environment while navigating [Inc18].

3. The Robotics Education \& Competition (REC) Foundation is interested in motivating students into science, technology, engineering, and mathematics (STEM) fields through the VEX Robotics Competition. Their goal is also to develop a handson and affordable curriculum in robotics programs in the U.S. and internationally targeting professionals and teachers who are interested in integrating computer science, computational thinking and robotics into their classrooms. This sort of initiative promotes learning STEM concepts while increasing teamwork, leadership, communications and problem solving skills.

Furthermore, the robotics industry is seeking to expand the availability of mobile robots in a multitude of applications. Google developed a self-driving car, Waymo (Figure 1.1(a)), equipped with sensors and cameras and they are writing a new chapter of artificial intelligence and robotics research. Waymo was developed to mimic how humans perceive objects on the streets and how this affects the way they drive and make decisions. These cars have driven over 5 million miles (mostly on city streets) [Inc18]. They are now expanding their service to more cities across the United States. Mobile robots also reached the air changing how agriculture is being done, through unmanned aerial vehicles, such as the DJI Matrice 210, (Figure 1.1(b)). This drone is able to avoid obstacles with its LIDAR sensor and map crops using their cameras and thermal imagery for surveillance and security. Moreover, their multispectral and hyperspectral sensors are able to detect and identify minerals and vegetation and to collect infrared radiation and ultraviolet light 
which are essential for assessing plant health, nutrient deficiencies, pest damage, foliar chemistry, surface chemical composition and water quality [Haw18]. Humanoid robots have also evolved since Piero Fiorito unveiled the first gigantic humanoid robot named Cygan in 1957. Boston Dynamics has developed Atlas (Figure 1.1(c)), which is a humanoid robot able to coordinate motions of the arms, torso and legs in order to mimic the movements of the human being. Moreover, through their sensors, Atlas is able to manipulate objects and walk on rough terrain [Dyn18a]. Boston Dynamics also developed an incredible mobile robot, named SpotMini (Figure 1.1(d)), which is a small four-legged robot that resembles a dog and is capable of picking up and handling objects using its arm and sensors. SpotMini also takes advantage of its stereo and depth cameras, an IMU, and position/force sensors to navigate indoors, avoiding obstacles [Dyn18b]. NASA's Curiosity rover (Figure 1.1(e)) is another example of a mobile robot that can perform outstanding tasks. It landed on Mars in 2012 and is able to identify microbial life using its 17 cameras, sensors and a robotic arm [NAS18]. NASA and California Institute of Technology are sending the Mars Helicopter (Figure 1.1(f)), which is a small and autonomous rotorcraft, to Mars in 2020. This shows the utility and the endless applications that mobile robots can have in order to explore and inspire a new generation of researchers to pursue a career in STEM fields [oTTJPL18b]. The i3XO EcoMapper autonomous underwater vehicle [Incb] is able to generate high-resolution maps of water quality, currents, bathymetry and sonar imagery (Figure 1.1(g)). This AUV is the one used for the data collection and experiments of this dissertation. More about this robot and its sensors will be discussed in Chapter. Another example of AUV is the AQUA2 (Figure 1.1(h)), which was developed by Adept Technology, Inc. and is used for coral reef monitoring, aquaculture and defense [OAM16]. Clearpath's Heron (Figure 1.1(i)) is a portable surface aquatic vessel which is able to collect water data e.g., temperature, salinity and $\mathrm{pH}$ through its sensors and accurate positioning [Rob18]. 


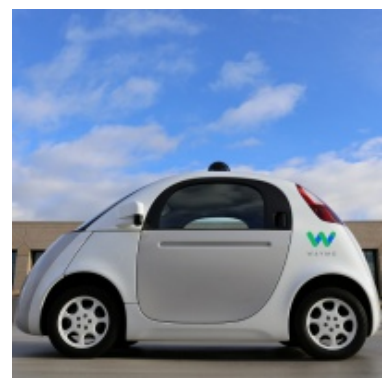

(a)

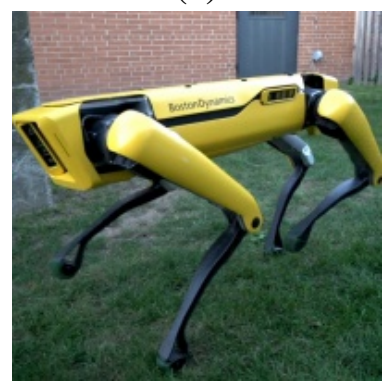

(d)

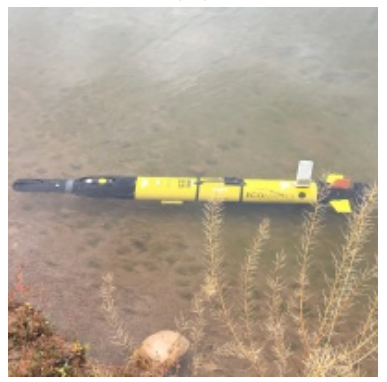

(g)

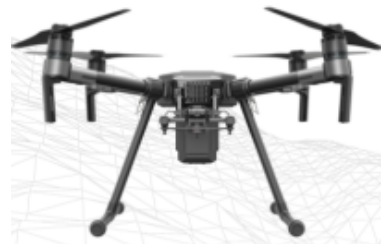

(b)

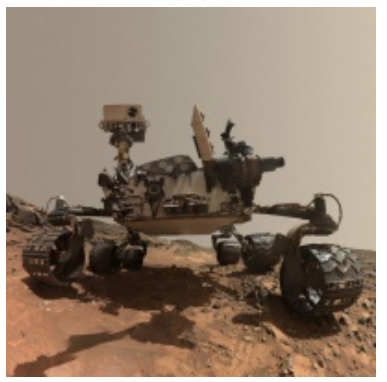

(e)

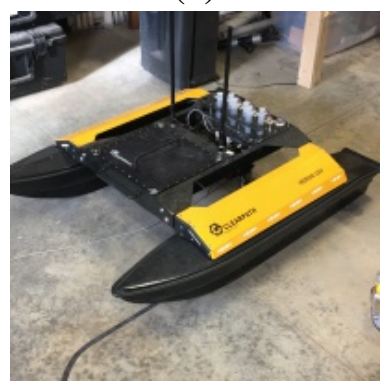

(h)

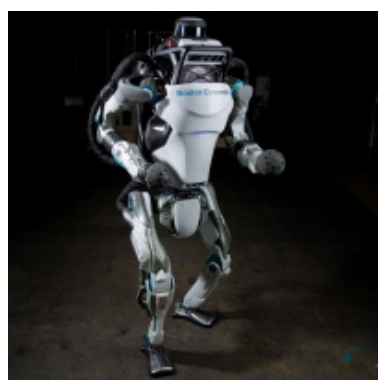

(c)

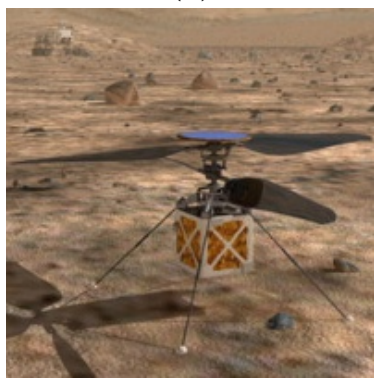

(f)

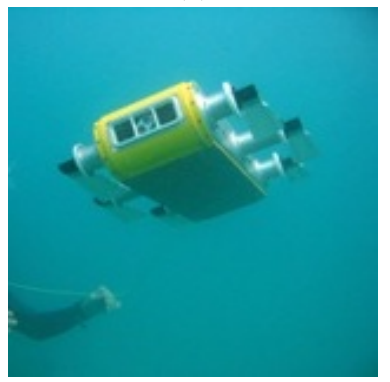

(i)

Figure 1.1: Examples of mobile robots: (a) The Google's Waymo self-driving car [Inc18]; (b) DJI Matrice 210 for precision agriculture [Haw18]; (c) Boston Dynamics's humanoid robot Atlas [Dyn18a]; (d) Boston Dynamics's dog-like robot SpotMini [Dyn18b]; (e) NASA's Curiosity rover for Mars exploration [oTTJPL18a] and [Ima18]; (f) The NASA's Mars Helicopter [oTTJPL18b]; (g) The YSI Ecomapper autonomous underwater vehicle (AUV) [Incb]; (h) The Clearpath's Heron autonomous surface vessel (ASV) [Rob18]; (i) AQUA2 Autonomous Underwater Vehicle [OAM16].

\subsection{Localization of Autonomous Underwater Vehicles}

Autonomous underwater vehicles (AUVs) suffer from GPS errors due to the electromagnetic waves that undergo attenuation in water. Therefore, in the absence of an external reference at known positions, the underwater vehicle has to rely on previous infor- 
mation obtained through a compass, inertial navigation system (INS), Doppler velocity logs (DLV), ultrashort-baseline (USBL) and long-baseline (LBL) acoustic positioning systems. Nonetheless, according to [BLF09], the error in the position estimate based using dead-reckoning information grows without bound. The average error in navigation is between $0.5 \%$ to $2 \%$ of distance traveled for underwater vehicles that operates within a few hundred meters of the sea floor [BLF09]. It is possible to achieve an error as low as $0.1 \%$, using large inertial navigation system; however, the cost for such infrastructure is high. By navigating via dead reckoning using an accelerometer, magnetometer and depth sensor for feedback, the vehicle is subject to large drift, leading to unbounded uncertainty in location. Coupled with the dynamics of the environment (specially in the ocean), a state estimate of location can deviate significantly from the actual location; sometimes on the order of kilometers, where errors can be as high as 20\% [BLF09].

\subsubsection{Solutions to the Problem of Localization}

The spatio-temporal dynamics of the ocean environment, coupled with limited communication capabilities, make navigation and localization difficult, especially in coastal regions where the majority of interesting phenomena occur. To add to this, the interesting features are themselves spatio-temporally dynamic, and effective sampling requires a good understanding of vehicle localization relative to the sampled feature. Furthermore, these interesting phenomena are usually identified by unique features in the ocean, e.g. significant bathymetric relief, an unstratified water column, or significantly different physical water parameter values. Here, we are interested in the utility of these unique features to aid in localization of underwater vehicles.

For example, autonomous gliders are a common tool used by ocean scientists to study a range of phenomena in the coastal and deep ocean $\left[\mathrm{SKA}^{+} 07, \mathrm{RDE}^{+}, \mathrm{JCG}^{+} 05 \mathrm{a}\right.$, 
$\left.\mathrm{CMG}^{+} 02 \mathrm{a}\right]$. More recently, at least $12 \%$ of the world's oceans that are covered by fixed or moving ice are still inaccessible to ocean science; therefore, AUVs are being used for this new class of underwater navigation [BW16]. One of the main problems with this application is the navigation and recovery of the vehicles, which motivates the studies of new techniques for localization and navigation of AUVs in GPS-denied environments. Autonomous gliders typically spend $8+$ hours underwater, navigating with only a compass, magnetometer and depth sensor. Increasing the surfacing frequency for location fixes/updates limits the amount of data that are collected during a deployment by decreasing the total time underwater, and by expending excess energy for communication and localization while on the surface [SKS12b]. Additionally, surfacing in potentially hazardous locations (e.g. shipping lanes) puts the vehicle at risk [PBHS13]. Hence, there is a trade-off between navigation accuracy and data collection and safety for the vehicle that must be considered for each mission. Thus, there is a need to increase navigation accuracy while keeping the vehicle underwater as long as possible. Potential solutions with high-powered sensors (e.g. Doppler Velocity Loggers) are feasible, however these also limit the deployment time by utilizing key power resources on-board the vehicle.

One way to localize autonomous underwater vehicles is to have them surface in order to obtain a position update through its GPS, but this is impossible (under ice) or undesirable for many applications. The use of static beacons in the form of a Long Baseline (LBL) array limits the operation area to a few kilometers squared and requires a substantial deployment effort before operations, especially in deep water.

Two common methods of correcting this issue are 1) surface more frequently for a GPS fix, or 2) integrate more accurate, energy intensive sensors, such as Doppler velocity loggers (DVLs). Both of these methods have drawbacks. Continually surfacing for a GPS fix takes away from sampling time and requires that more energy be used for communica- 
tions. Surfacing also poses a physical threat to the vehicle, as it might accidentally surface in a hazardous location, e.g. a shipping lane. Using more powerful sensors consumes the finite energy supply of an AUV faster and significantly reduces the deployment duration. To optimize time spent collecting data with these vehicles, it is desirable to find alternative means of reducing position uncertainty while underwater. Here, we approach the problem by using existing sensors and data gathered in situ by augmenting the technique of terrain-based navigation.

Even with higher resolution bathymetric maps, traditional terrain-based navigation methodologies can result in significant navigational error, especially in regions of little to no vertical relief. To enhance the ability to navigate and localize, we have developed an augmented TBN that incorporates physical science data, i.e. water parameters such as temperature, salinity, $\mathrm{pH}$, etc., to enhance the topographic map that the vehicle uses to navigate under the traditional $\mathrm{TBN}$ framework $\left[\mathrm{RFB}^{+} 17 \mathrm{a}\right]$. In this navigation scheme the bathymetric data are combined with the physical science data to enrich the uniqueness of the underlying terrain map. This method of localization has been evaluated with data gathered at multiple locations in both lake and ocean environments. Results from a deployment in the Big Fisherman's Cove, Santa Catalina Island is presented in $\left[\mathrm{RFB}^{+} 17 \mathrm{a}\right]$; these and other preliminary results from our Augmented Terrain Based Navigation (ATBN) have been promising.

Effective observation and quantification of spatiotemporally dynamic processes occurring in aquatic environments, e.g., the ocean, requires simultaneous measurement of diverse water properties, which must be made rapidly to capture the both the spatial and temporal variability of multiple simultaneous interactions. This cannot be done by traditional oceanographic methods involving infrequent and sparse measurements from ships, buoys and drifters. We must employ an adaptive-sampling, heterogeneous team of robotic assets that can perform in situ feature recognition and event response with accurate local- 
ization to plug a substantial gap in our understanding of a range of processes: physical (e.g., tidal mixing and seasonal overturn), chemical (e.g., nutrient upwelling and hypoxia), and biological (e.g., harmful algal blooms). Successfully orchestrating a multi-vehicle, deployment additionally requires a robust, rapid and cost-effective communication network. Only when all these components, which form an aquatic robotic sensing system, are in synchronous operation can scientists begin to improve our overall understanding of the complex aquatic environment.

By creating novel localization algorithms, either a global localization where no previous information of the state of vehicle is given or a tracking problem, where a previous GPS information is part of the algorithm's input, and through the development of an augmented terrain based framework that combines physical water parameters to the bathymetric information, the present dissertation advances the areas of robotics and ocean sciences. The techniques presented in the following chapters have application in underwater navigation for GPS-denied environments, ground applications for mobile robots, precision agriculture, localization of unmanned aerial vehicles and modeling of several physical phenomena in aquatic environments (saline or freshwater). Other contributions lie in the fact that by enabling a persistent and more reliable (accuracy in localization) navigation of underwater vehicles, our understanding of the dynamics of the ocean, as well as its monitoring and security, can be expanded and new questions will most likely be asked.

\subsection{Organization of the Dissertation}

We conclude this introductory chapter with a preview of the remainder of the dissertation. Chapters 3, 4, 5 and 6 contain original contributions. Concluding remarks, open problems, and some potential avenues for future work appear in Chapter 7. The structure 


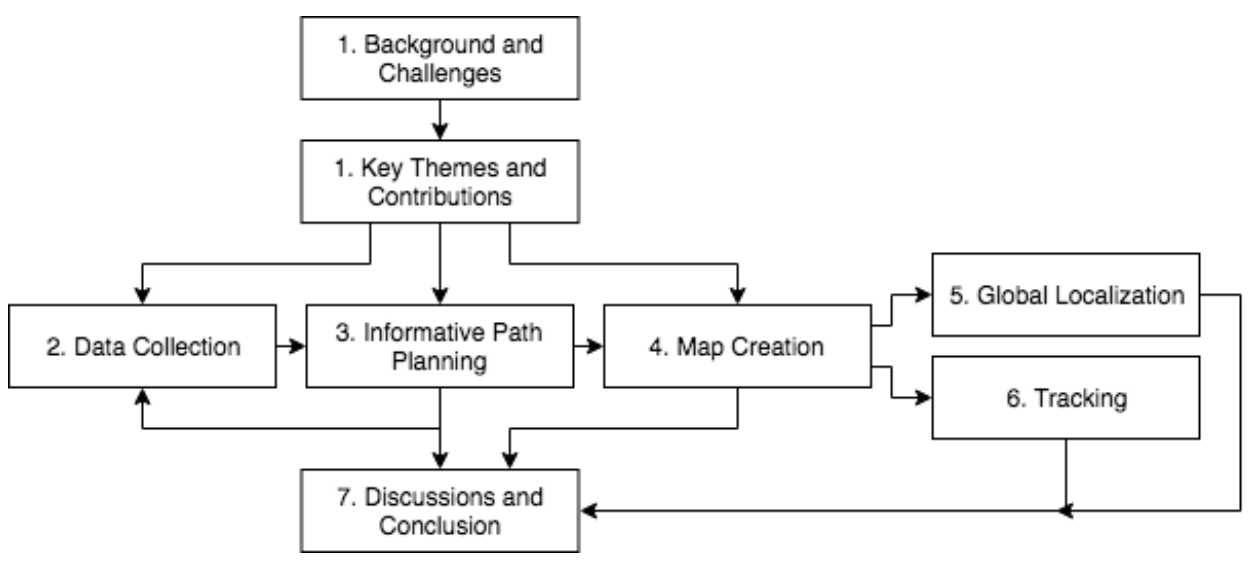

Figure 1.2: Organization of this dissertation with arrows indicating dependencies. and dependencies between chapters are shown in Figure 1.3. The contributions of this dissertation are laid out in the following chapters as follows:

- Chapter 2 We describe the data collection process in Section 2.1 and the water parameters which we were interested in analyzing to aid in localization of underwater vehicles. A brief overview of the autonomous underwater vehicle AUV utilized throughout this dissertation is given in Section 2.3. Section 2.4 provides a short overview and the motivation of the environments where we collected and analyzed the data used in this research.

- Chapter 3 We examine different interpolation methodologies for the process of creating terrain maps for each water parameter. Then, we propose a sampling-based framework to decrease the errors in prediction of reference maps for navigation using Markov Decision Processes (MDP) for generating optimal strategies in sampling.

- Chapter 4 We present a novel approach to augment terrain-based navigation methods by combining bathymetric data with physical water parameter data. The goal is to update state-of-the-art terrain maps to provide maximal utility to underwater vehicles for reducing uncertainty in a navigation solution. We examine the com- 
puted weighting scheme over space and time of the incorporated parameters for both ocean and fresh water locations that were sampled at multiple times.

- Chapter 5 We implemented a novel, global localization algorithm in GPS-denied environments. The algorithm is used to localize a list of observations collected by the underwater vehicle in the region where the terrain map was created. We present tests of this methodology on multiple datasets from field trials and show two the deployments we used in this work in the Big Fisherman's Cove, Santa Catalina Island).

- Chapter 6 We introduce a new application for the whitening process in a marine environment. We developed a new method for localizing the state trajectory of an AUV by non-parametrically combining the bathymetric information with water sensor data e.g., dissolved oxygen concentration, temperature, and turbidity. By applying a zero-phase components analysis (Mahalanobis whitening), we were able to reveal the true distances between data-points and to localize with higher precision. 


\section{CHAPTER 2}

\section{DATA COLLECTION}

In this chapter, the focus is on the locations where the data used in this research were collected and the motivation behind the chosen environments. Moreover, a brief overview of the autonomous underwater vehicle used in the data collection will be presented. Data for this research were collected at three locations: (a) USC Wrigley Institute for Environmental Studies, Santa Catalina Island, California, USA; (b) Lake Nighthorse, Durango, Colorado; and (c) Moss Landing, Monterey Bay, California. The datasets were collected on the water surface at a rate of $2 \mathrm{~Hz}$ using the YSI Ecomapper underwater vehicle (Figure 2.2). The sampling areas were covered by running dense lawnmower patterns for total spatial coverage.

\subsection{Water Parameters}

The physical water parameters measure at each location were Temperature $\left(\mathrm{Temp},{ }^{\circ} \mathrm{C}\right)$, Salinity (Sal, ppt), pH, Percent Dissolved Oxygen (ODO, mg/L), Blue Green Algae (BGA, PC cells/mL), Turbidity (Turbid, NTU), Total Water Depth (Depth, m), and Chlorophyll (Chl).

\subsubsection{Temperature}

Temperature of water is arguably the most common and important property since most of the other water parameters depend on temperature for accuracy [Incc]. According to the Encyclopaedia Britannica [oEB18], temperature is a "measure of hotness or coldness expressed in terms of any of several arbitrary scales and indicating the direction in which heat energy will spontaneously flow i.e., from a hotter body (one at a higher temperature)

to a colder body (one at a lower temperature)". By having an accurate measurement of 
temperature, it is possible to identify changes in the thermocline which affect the health of species and organisms of the water [Incc]. The AUV used in this research measures temperature using a high-precision thermistor sensor and the unit used in the following chapters is degrees Celsius $\left({ }^{\circ} \mathrm{C}\right)$.

\subsubsection{Salinity}

Salinity is the measurement of the concentration of salts dissolved in water. It is known that even small variations in salinity can lead to significant effects on the cycles and circulation of the ocean. The salt that we can find in the ocean is made up of sodium (Na) and chlorine $(\mathrm{Cl})$ in its majority, accounting for more than $90 \%$ of the dissolved salt. However, it is also possible to encounter potassium $(\mathrm{K})$, magnesium $(\mathrm{Mg})$ and calcium (Ca) in the marine environment. Salinity values are determined through the Practical Salinity Scale (ppt). Sometimes it is possible to find it in psu (Practical Salinity Units) or percentage $(\%)$.

\subsubsection{Dissolved Oxygen}

The dissolved oxygen is a measurement of the level of oxygen $\mathrm{O}_{2}$ that is not bonded to any other element and is present in liquids (such as water). Most living organisms depend on oxygen for their survival; therefore, an appropriate level of dissolved oxygen is crucial for the evaluation of water quality and marine ecosystems [Inca].

In water, dissolved oxygen is originated through the air (surrounding atmosphere) or as a waste product of photosynthesis from aquatic plants e.g., phytoplankton, algae, and seaweed [Inca]. 


\subsection{4 pH}

The power of hydrogen is a measurement that compares the relative acidity or alkalinity nature of a solution at a certain temperature [Inc14a]. It is measure in a scale from 0 to 14, where 7 represents a neutral solution; lower levels represent a more acidic solution (activity of hydrogen ions is greater than the activity of the hydroxide ions); and higher levels represent a more basic (or alkaline) solution (activity of hydroxide ion is greater than the activities of the hydrogen ions) [Inc14a].

\subsubsection{Turbidity}

Turbidity is the measurement that deals with how clear the water is ("murkiness" or "cloudiness"). Water quality is directly affected by suspended sediments e.g., particles of clay, soil and silt that may contain pollutants e.g., phosphorus, pesticides or heavy metals. A high level of concentration of such pollutants interferes on the quantity of light that penetrates the body of water [Incd].

Measuring turbidity of the water is important to evaluate the health of the water body. A quick change in turbidity levels can dangerously affect the ecosystem and can be an indication of poor water quality [Incd]. Having an updated dataset of historical turbidity dataset is crucial when monitoring a certain area. For most sensors, turbidity is measured in Nephelometric Turbidity Units (NTU) or Formazin Nephelometric Units (FNU). It is measured using a white light at a 90 degree detection angle (ISO7027 compliant) [Incd].

The AUV used in this research measures turbidity with an optical sensor, where light from the emitter enters the sample and scatters off particles in the water. 


\subsubsection{Chlorophyll}

Chlorophyll can be understood as a color pigment found within living cells of plants, algae and phytoplankton. It is the key biochemical components in the photosynthesis process as a photoreceptor [FE14]. Chlorophyll specifically absorbs energy from sunlight [Spe97]. The fact that plants and algae appear green is because chlorophyll reflects the green wavelengths found in sunlight and absorbing all other colors [FE14]. There are 6 different chlorophylls identified, each reflect different ranges of green wavelengths [Wet01] and [Cal10]. Chlorophyll A is the primary molecule responsible for photosynthesis and is found in every single photosynthesizing organism [Wet01] and [Spe97]. Chlorophyll B is found in land plants, aquatic plants and green algae [Wet01]. Chlorophyll assists carbon dioxide to be reduced by water in the following photosynthetic reaction below:

$$
6 \mathrm{CO}_{2}+6 \mathrm{H}_{2} \mathrm{O} \stackrel{\text { Sunlight and chlorophyll }}{\longrightarrow} \mathrm{C}_{6} \mathrm{H}_{12} \mathrm{O}_{6}+6 \mathrm{O}_{2}
$$

In addition, chlorophyll is important for the existence of phytoplankton, which, in turn, is an indicator of water health. It is also known that when high levels of chlorophyll are found on surface waters, this indicates a high concentration of nutrients e.g., phosphorus and nitrogen [Inc14b].

Chlorophyll is measured in micrograms per liter $(\mu \mathrm{g} / \mathrm{L})$. The techniques used to measure chlorophyll include spectrophotometry, high performance liquid chromatography (HPLC), and fluorometry. In the latter, the sensors used to measure chlorophyll use fluorescence to estimate the levels of phytoplankton based on chlorophyll concentrations in a sample of water [Inc14b]. Fluorescence can be understood as the a lower energy light that is emitted when chlorophyll is exposed to a high-energy wavelength (approximately $470 \mathrm{~nm})[\mathrm{Inc} 14 \mathrm{~b}]$. 


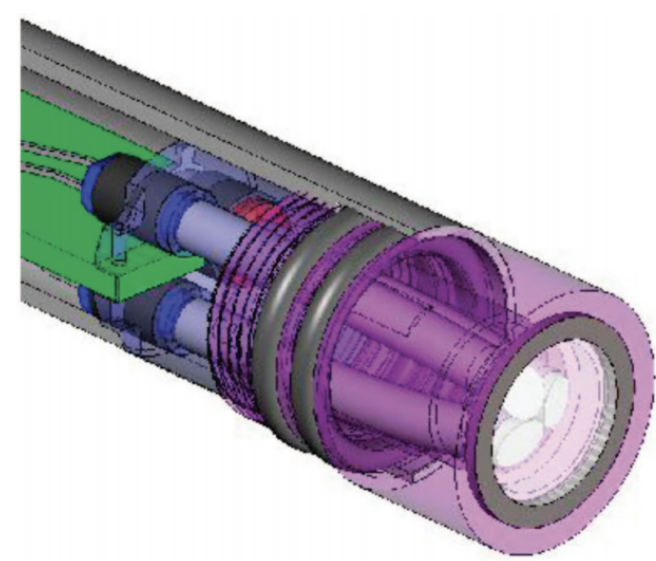

(a)

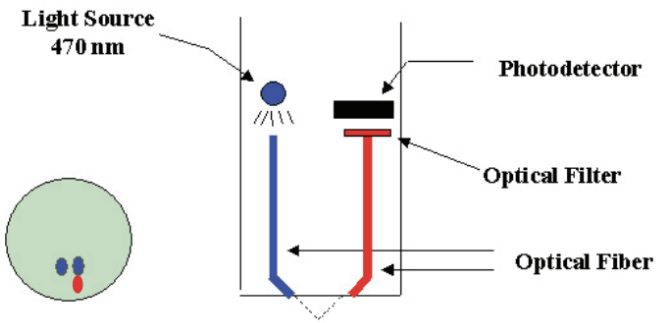

(b)

Figure 2.1: (a) Diagram of YSI 6035 chlorophyll crobe and (b) Diagram of EXO total algae (Chlorophyll + Blue-green Algae) probe. Images obtained from the Tech Note on The Basics of Chlorophvll Measurement in [Inc14hl.

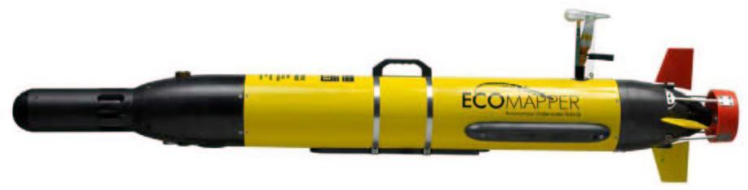

Figure 2.2: The YSI Ecomapper underwater vehicle used in the data collection of this work.

\subsection{The autonomous underwater vehicles (AUVs)}

The YSI Ecomapper is an autonomous underwater vehicle for collecting water quality data. The i3XO EcoMapper AUV (Autonomous Underwater Vehicle) used in this research for collecting data contains the EXO sensors that are able to measure water quality, currents, and bathymetric information at a continuous interval for missions that range from 8-12 hours long [Incb].

According to the datasheet of this vehicle [Incb], it is possible to identify several advantages e.g., it is easily deployed by one person; geo-referenced data; can measure up to 8 different water quality parameters; it is rugged and is built in lightweight carbon fiber and marine-grade aluminum; powered with Li-Ion batteries and it contains built-in moisture detectors. 


\subsubsection{Santa Catalina Island, CA, USA}

The Big Fisherman's Cove is a marine protected area adjacent to the University of California's Wrigley Marine Science Center. It is a conservation area for habitats and organisms protection. Our interest in this area comes from the fact that due to the increase rate of urbanization in coastal areas, the quantity and quality of freshwater are usually altered. Furthermore, this can have major impact on particulate and solute loadings in these regions. It is known that these changes led into an increase occurrence of algal and phytoplankton blooms [WF04].

These biological phenomena are a primary research interest of several scientists and engineers $\left[\mathrm{SCL}^{+} 10 \mathrm{a}\right]$. The authors assessed and evaluated the potential prediction of harmful algal species (i.e. harmful algal blooms (HABs)). It is still under investigation what triggers and help disseminate $\mathrm{HAB}$ events in the oceanic environment. Therefore, the authors chose to give special attention to Southern California, where not only HABs have been identified, but also due to the significant variability associated with the Pacific decadal oscillation (PDO) and the El Niño southern oscillation (ENSO) ([DRA93] and $\left.\left[\mathrm{KTK}^{+} 02\right]\right)$. An oceanic region contained within $32^{\circ} \mathrm{N}$ to $34.5^{\circ} \mathrm{N}$ and $-117^{\circ} \mathrm{E}$ to $-121^{\circ}$ E is under continued study to uncover the connections between small-scale biophysical processes and large-scale events related to algal blooms, specifically blooms composed of toxin-producing species (i.e., HABs) [JND02], [SMS $\left.{ }^{+} 07\right]$ and [SDH $\left.{ }^{+} 10\right]$.

These phenomena play a major role in the prediction of regional storm events and in the physical and biogeochemical dynamics of the coastal marine ecosystem [SCL $\left.{ }^{+} 10 \mathrm{a}\right]$. Moreover, a freshening of sea-surface waters have been identified due to an increase in rainfall in an urban, coastal region results in through direct rainfall into the ocean and from freshwater inflow from streams and rivers. These rivers carries nutrients and toxins into the water that can directly impact the increase in algal blooms. What researchers still seek to discover is whether it is possible to differentiate between anthropogenically 


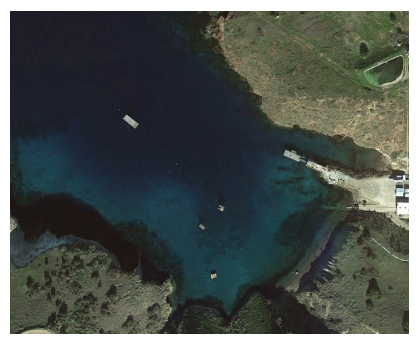

(a)

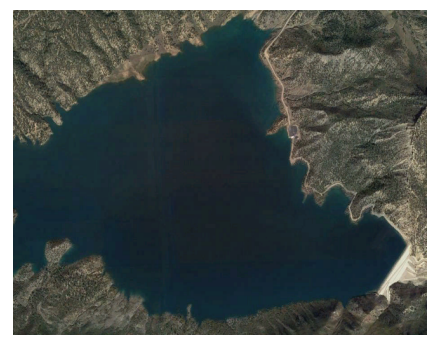

(b)

Figure 2.3: Aerial Image of the Big Fisherman's Cove, Santa Catalina Island, CA, USA (a) and the Lake Nighthorse, CO, USA (b)

affected processes and natural variations and effects in the coastal regions. This motivates the use of autonomous underwater vehicles in the data collection and monitoring of these areas.

The data used in the following chapters were in the Big Fisherman's Cove, Santa Catalina Island $\left(33^{\circ} 26^{\prime} 40.4^{\prime \prime} \mathrm{N}, 118^{\circ} 29^{\prime} 6.5^{\prime \prime} \mathrm{W}\right)$ on July $13-14,2016$. The position of the Big Fisherman's Cove is on the northern coast of the island and is primarily a shallow, protected, saline ocean bay. All vehicle deployments were near shore over both sandy and coral substrate that was $<40$ m deep. Two total-coverage datasets of the entire cove were taken on July 13, between 3:25pm and 7:30pm (local time UTC -08.00). Four datasets were taken the next day, between 5:11pm and 6:50pm, near the shoreline southwest of the Wrigley Research Institution's dock. All of these data were collected while the robot was running on the surface to provide accurate position estimation via GPS.

\section{Descriptive Statistics}

A descriptive statistics of the datasets utilized for the remaining of the chapters is presented here. The minimum, maximum, mean and standard deviations of the sensor data collected during the first mission at the Big Fisherman's Cove, Santa Catalina Island, CA, USA, starting at 14:46:15 (MST) and ending 15:16:12 (MST) can be found in 2.1 and the for the second mission can be found in 2.2. 


\begin{tabular}{|c|c|c|c|c|}
\hline & Min & Max & Mean & Std \\
\hline Latitude & 33.444 & 33.446 & 33.445 & 0.000 \\
\hline Longitude & -118.486 & -118.484 & -118.485 & 0.001 \\
\hline Depth & 0.310 & 38.680 & 13.401 & 7.331 \\
\hline Temperature $\left({ }^{\circ} \mathrm{C}\right)$ & 21.700 & 23.640 & 22.350 & 0.425 \\
\hline Salinity $(\mathrm{ppt})$ & 5.990 & 34.010 & 33.821 & 0.667 \\
\hline $\mathrm{pH}$ & 8.160 & 8.560 & 8.391 & 0.052 \\
\hline Turbidity (NTU) & 0.100 & 3.200 & 1.329 & 0.474 \\
\hline Dissolved Oxygen (mg/L) & 8.120 & 9.500 & 8.481 & 0.295 \\
\hline
\end{tabular}

Table 2.1: Descriptive statistics for the dataset collected during a mission at the Big Fisherman's Cove, Santa Catalina Island, CA, USA, on July 13th 2016

\begin{tabular}{|c|c|c|c|c|}
\hline & Min & Max & Mean & Std \\
\hline Latitude & 33.444 & 33.445 & 33.444 & 0.000 \\
\hline Longitude & -118.486 & -118.484 & -118.485 & 0.000 \\
\hline Depth & 0.870 & 11.930 & 4.865 & 1.993 \\
\hline Temperature $\left({ }^{\circ} \mathrm{C}\right)$ & 21.870 & 23.290 & 22.395 & 0.256 \\
\hline Salinity (ppt) & 1.170 & 34.020 & 33.784 & 1.110 \\
\hline $\mathrm{pH}$ & 8.300 & 8.520 & 8.405 & 0.034 \\
\hline Turbidity (NTU) & 0.100 & 3.000 & 1.302 & 0.463 \\
\hline Dissolved Oxygen (mg/L) & 8.170 & 9.860 & 8.694 & 0.362 \\
\hline
\end{tabular}

Table 2.2: Descriptive statistics for the dataset collected during a mission at the Big Fisherman's Cove, Santa Catalina Island, CA, USA, on July 14th 2016.

\subsubsection{Lake Nighthorse, CO, USA}

The Lake Nighthorse $\left(37^{\circ} 13^{\prime} 13.4^{\prime \prime} \mathrm{N} 107^{\circ} 53^{\prime} 53.7^{\prime \prime} \mathrm{W}\right)$ is a fairly recent reservoir whose project started back in the 60s and was opened to public in April 2018. When the reservoir started being built, the valley located at that site was the center of local Native Americans that inhabited the location approximately one thousand years ago; thus it is a historical/archaeological site that remains underneath the water. Moreover, the lake is pristine in the sense that by being a new reservoir there are no invasive species. At other lakes, invasive species are known to destroy the ecology, affecting its health. The interest in the Lake Nighthorse also comes from the desire to maintain a healthy ecology while still 
allowing public to use for example for recreational purposes. Another interesting point to highlight is that every summer, the reservoir is refilled from different sources, which may alter parameters e.g., temperature, salinity, $\mathrm{pH}$, dissolved oxygen and especially turbidity every time it is refilled. One question one might ask is if the refilling process is changing the chemistry of the lake, if it is allowing invasive species to reproduce and establish in that environment and if the health of the lake is being compromised by the previous two changes. The Lake Nighthorse is a large freshwater reservoir with no major natural inflows and no outflow. The lake serves as a contingency freshwater supply for the town of Durango, CO, and as such, remains stagnant for most of the year outside of spring inflow. This lake can simulate an ocean environment in the sense that its dynamics allow the occurrence of large scale phenomenon and features and allow the aquatic robots to navigate in such a long trajectory that this robot can be lost. Therefore, the lake is small enough that it is possible to see its other side, but big enough that a robot can be lost. The sampling area was a portion of the reservoir that was parallel to the dam wall. The Ecomapper ran on the surface for the entire sampling to provide accurate position estimation via GPS.

\section{Descriptive Statistics}

A descriptive statistics of the datasets utilized for the remaining of the chapters of this dissertation can found in this subsection the minimum, maximum, mean and standard deviation of the sensor data collected during the first mission at the Lake Nighthorse, CO, USA starting at 14:46:15 (MST) and ending 15:16:12 (MST) on April 25th 2018. 


\begin{tabular}{|c|c|c|c|c|}
\hline & Min & Max & Mean & Std \\
\hline Latitude & 37.236 & 37.239 & 37.237 & 0.001 \\
\hline Longitude & -107.913 & -107.908 & -107.910 & 0.001 \\
\hline Depth & 0.230 & 18.010 & 6.312 & 3.349 \\
\hline Temperature $\left({ }^{\circ} \mathrm{C}\right)$ & 7.110 & 14.610 & 10.430 & 1.200 \\
\hline Salinity $(\mathrm{ppt})$ & 0.090 & 0.230 & 0.220 & 0.004 \\
\hline $\mathrm{pH}$ & 8.230 & 8.550 & 8.412 & 0.066 \\
\hline Turbidity (NTU) & 0.100 & 177.800 & 3.108 & 17.293 \\
\hline Dissolved Oxygen $(\mathrm{mg} / \mathrm{L})$ & 9.320 & 10.070 & 9.764 & 0.105 \\
\hline
\end{tabular}

Table 2.3: Descriptive statistics for the dataset collected during a mission at the Lake Nighthorse, CO, USA, on April 25th 2018, in the afternoon.

On May 2 2018, two different missions were executed, one in the morning starting at 09:21:54 (MST) and ending at 12: 37:58 (MST) and one in the afternoon starting at 12:38:38 (MST) and ending at 15:47:14 (MST).

\begin{tabular}{|c|c|c|c|c|}
\hline & Min & Max & Mean & Std \\
\hline Latitude & 37.236 & 37.239 & 37.238 & 0.001 \\
\hline Longitude & -107.912 & -107.909 & -107.910 & 0.001 \\
\hline Depth & 0.230 & 12.790 & 5.436 & 2.841 \\
\hline Temperature $\left({ }^{\circ} \mathrm{C}\right)$ & 8.930 & 10.560 & 10.283 & 0.321 \\
\hline Salinity $(\mathrm{ppt})$ & 0.010 & 0.220 & 0.212 & 0.014 \\
\hline $\mathrm{pH}$ & 8.080 & 8.560 & 8.416 & 0.083 \\
\hline Turbidity (NTU) & 0.100 & 11.800 & 2.752 & 2.472 \\
\hline Dissolved Oxygen (mg/L) & 9.210 & 9.560 & 9.396 & 0.046 \\
\hline
\end{tabular}

Table 2.4: Descriptive statistics for the dataset collected during a mission at the Lake Nighthorse, CO, USA, on May 2nd 2018, in the morning. 


\begin{tabular}{|c|c|c|c|c|}
\hline & Min & Max & Mean & Std \\
\hline Latitude & 37.236 & 37.239 & 37.238 & 0.001 \\
\hline Longitude & -107.913 & -107.909 & -107.910 & 0.001 \\
\hline Depth & 0.230 & 14.740 & 4.411 & 2.717 \\
\hline Temperature $\left({ }^{\circ} \mathrm{C}\right)$ & 8.540 & 11.000 & 10.272 & 0.409 \\
\hline Salinity $(\mathrm{ppt})$ & 0.030 & 0.220 & 0.209 & 0.011 \\
\hline $\mathrm{pH}$ & 8.170 & 8.550 & 8.392 & 0.058 \\
\hline Turbidity (NTU) & -0.700 & 1057.500 & 3.614 & 18.706 \\
\hline Dissolved Oxygen $(\mathrm{mg} / \mathrm{L})$ & 9.310 & 9.810 & 9.428 & 0.070 \\
\hline
\end{tabular}

Table 2.5: Descriptive statistics for the dataset collected during a mission at the Lake Nighthorse, CO, USA, on May 2nd 2018, in the afternoon.

\subsubsection{Monterey Bay, CA}

A single dataset was used from Monterey Bay (Fig ??-c), collected just offshore from the

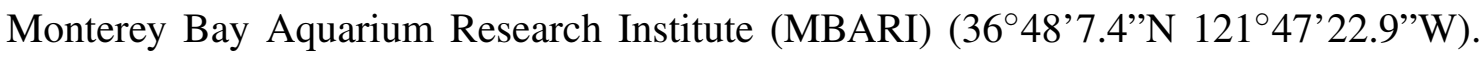
The deployment surveyed a rectangular area about a half mile in length right in front of MBARI. The surveyed area was a coastal oceanic bay with saline water, and the total water depth in the surveyed region was generally $\sim 50 \mathrm{~m}$. This dataset was collected on December 20, 2012 at 2:56pm (local time UTC -08.00). The robot performed an undulating pattern during this deployment, constantly diving and surfacing between the surface and $14 \mathrm{~m}$ depth, surfacing occasionally for a GPS fix. 


\section{CHAPTER 3}

\section{INFORMATIVE PATH PLANNING}

\subsection{Overview}

As a first step, we look into the data collection process, starting in surveying different bodies of water, such as lakes and coastal regions of the ocean (see Chapter 2). In this chapter, we are interested in dealing with the pre-processing of the data, starting from predicting observations in locations where the robot did not traverse and ending figuring out what are the optimal path to execute to collect data for decreasing the errors in prediction. By using the collected data, we interpolate and construct heat maps for each one of the parameters to be used in the development of the underlying terrain maps. Next, we examine the computed weighting scheme of the incorporated parameters $\left[\mathrm{RFA}^{+} 17 \mathrm{a}\right]$ for both ocean and fresh water locations (see Chapter 4). When an aquatic vehicle is executing a mission (e.g., an exploration, a survey, etc), new water information is being collected and compared to these pre-designed maps in that specific region. The motivation for this analysis is to either a) validate that a previously computed map is valid for a new deployment, or b) update a previous map for current use. In either case, we are interested in updating this terrain map to provide maximal utility to underwater vehicles for reducing uncertainty in a navigation solution. As seen before, underwater vehicles commonly perform navigation via dead reckoning using position sensors which can cause a significant deviation. For this reason, we aim at developing algorithms for localization of aquatic vehicles using the informative terrain maps developed. Moreover, interesting phenomena on the coastal region of the ocean are usually identified by unique features in the ocean, e.g., significant bathymetric relief, an unstratified water column, or significantly different physical water parameter values. Here, we are interested in the utility of these unique features to aid in localization and navigation for underwater vehicles. By experimentally 
testing our localization methods and navigate different autonomous water vehicles, we are able to capture the range of spatial and temporal scales related to the water parameters. These deployments were executed on different days over different bodies of water.

We focus on the processing of the data collected by the robots and the create of the underlying terrain maps. The proposed method for increasing navigational accuracy and reducing uncertainty in navigation solutions is named Terrain-Based Navigation (TBN), which was developed prior to satellite-based navigation, e.g., GPS, [Gol80a]. The navigational accuracy of this method is dependent upon the resolution of the underlying topography map and the accuracy of the elevation measurement; both good for terrestrial applications. This system became redundant after the introduction of GPS, although it is still a useful navigational aid for GPS-denied environments, e.g., underwater. Until recently, the utility of this TBN for underwater vehicles was low due to the poor resolution of bathymetric maps; however, updated bathymetry maps with higher resolution provide motivation for revisiting the application of this method for low-power, accurate navigation underwater, see [SKS16]. One clearly identified shortcoming of TBN in the aquatic environment is the lack of accurate, high-resolution maps of the sea floor in many regions. Additionally, sensor limitations, especially the limitations of optical range sensors, substantially restrict TBN underwater. In [KEW06], it is concluded that improved navigation will enable new missions that would previously have been considered infeasible or impractical.

An approach to implementing a traditional terrain-based navigation methodology for localization is described in [SKS16]. In this work, maps provided by the Southern California Coastal Ocean Observing System were used for comparison with the depth data collected by the Slocum gliders. One of the biggest problems was the resolution of the maps, a 30 arc second grid, which was still too low to accurately localize from. Studies like this one and other field studies all highlight the fact that the practice of creating a 
detailed bathymetric map of the survey area would improve the ability to localize and navigate with TBN. Nevertheless, even with higher resolution bathymetric maps, traditional TBN alone can result in significant navigational error, especially in regions of little to no vertical relief. To enhance the ability to navigate and localize, we developed an Augmented TBN that incorporates physical science data, i.e., temperature, salinity, pH, etc., to enhance the topographic map that the vehicle uses to navigate under the traditional TBN framework $\left[\mathrm{RFA}^{+} 17 \mathrm{~b}\right]$. In this navigation scheme the bathymetric data are combined with the physical science data to enrich the uniqueness of the underlying terrain map.

We rely on the concept of Environmental or Ecological Niche Models (see Chapter 4) for our assumption that including physical water data into the terrain map provides a reliable model for localization. Ecological Niche Modeling is derived from one of the main goals of ecology, which is to map species distribution over geographic ranges and be able to use predictive models to infer where various species are likely to be found [MM15][MTMT09]. Most localization approaches in the field of Robotics are based on recursive Bayesian filters, e.g., Kalman filters (including EKF and UKF) [LDW91a, JLV99a], and particle filters [TFBD01, Die03]. However, these approaches can be cost prohibitive for underwater vehicle missions due to their computation time and memory requirements, need for sophisticated sensors, and motion modeling. Our work is motivated by work on GPS-denied environment scenarios [EL13, EKOL08a, OL05, OL07a].

The ocean is a vast and complex environment and the most important question we can ask is when and where to sample so that the data is valuable for further understanding of the dynamically evolving phenomena that occur. Interesting phenomena can occur in large areas, making it difficult for traditional oceanographic methodologies to provide valuable information. For this, we need a diverse set of spatio-temporal measurements. Mobile robots play an important role since they can last as long as these phenomena occur. 
Several studies of the application of mobile robots have been published [Yuh00][BL02]. In the case of adaptive sampling techniques for AUVs, several works such as [SYB97a]-[EOL $\left.{ }^{+} 01\right]$ provide a strong background to the fiel. Other algorithms to position vehicles in the right place at the right time are shown in $\left[\mathrm{SSS}^{+} 11 \mathrm{a}, \mathrm{SCL}^{+} 10 \mathrm{~b}\right]$. Works on sensor placement can be seen in [ZS08]-[BL94]. Towards ocean fronts, humanin-the-loop methods for adaptive sampling can be found in $\left[\mathrm{GCE}^{+} 13, \mathrm{DMM}^{+} 11, \mathrm{MDM}^{+} 12\right]$, deliberative planning [Raj, RPB12], in situ reaction to collected data [ZRB $\left.{ }^{+} 12\right]-\left[\mathrm{GGM}^{+} 12\right]$, and predictive estimation from ocean models with multiple vehicles [RH14, RH13].

Existing investigations on robotic sampling have a fundamental link to a geographical coordinate system, i.e., latitude and longitude. It is important to highlight that the definition of geographical space is not well defined in the complex ocean dynamics. Geographic-relative navigation is difficult, especially when tracking and localizing dynamic events. To address the problem of uniformly distributed geographic coordinates using machine learning techniques and optimal sensor placement can be found in [KMGG08, KGGK06, KSG08, KG07, SKGK09]. The main problem here that we try to address in this dissertation is that all the previous works assumes that the scalar field is static. Here we are interested in investigating sampling and localization within a spatio-temporally dynamics of the ocean features.

\subsection{Trajectory Design with Predictive Ocean Models}

The use of predictive models in path planning in the ocean is not a new concept, as ocean current models have been widely utilized to solve path planning optimization problems, e.g., $\left[\mathrm{CMN}^{+}\right.$92]-[ACO04]. Of particular interest to the ocean robotics community is utilizing ocean currents to minimize energy consumption, thus extending a vehicle's deployment time. Additionally, complex current structures experienced in a coastal region 
can vary significantly with time and location, making subsurface navigation difficult. The authors of $\left[\mathrm{CMN}^{+}\right.$92]-[ACO04] address the problem of path planning for AUVs in a complex, time-dependent, variable ocean. However, the assumed current velocities are coarse resolution averages. As currents are estimated from a compiled database, they are either average conditions as seen over long time periods, or are provided only in two spatial dimensions. In this study, we will not only use high-resolution ocean models that output near real-time 4-D current velocities for path planning, but we also predict the structure and motion of the coherent ocean feature.

\subsection{Dealing with Uncertainty}

While incorporating different planning and decision paradigms with stochastic methods on-board autonomous vehicles is possible, it is not required that the planning system itself directly deal with probabilistic methods. Stochastic representations of the environment will be crucial to informing the search mechanisms embedded on-board the robots for feature detection and localization. Because of the temporal and spatial variability of ocean features, the location information determined by predictive models do not guarantee where the feature is actually located globally. Thus, the robots will require embedded methods for intelligent search to allow them to autonomously find and localize within the feature of interest.

There is a need to develop an acceptable boundary between in situ adaptability and near real-time, spatio-temporal knowledge of the entire survey area. This work challenges the traditional theme of dealing with exploration versus exploitation and adapts it to the oceanographic context. This novel approach can provide the robot an understanding or broad outline of what it should expect for the given sampling scenario, but the specifics of how a plan is executed and the on-board decisions will be left to the robot 
given the local dynamics and unique aspects of each feature. Methods for demonstrating the ability to detect a proof-of-concept ocean front using a YSI EcoMapper AUV in situ are validated and extended in a laboratory setting in [KHS15].

Environmental niche models $\left[\mathrm{EPH}^{+} 11\right]$ learn the relationship between a desired sample (e.g., organism abundance) and environmental conditions, providing an alternate way of targeting sample acquisition or navigating through space. Here,instead of thinking of locations existing in geographic space, we consider them to be drawn from or exist in an environmental space. This space contains environmental parameters e.g., temperature, salinity, $\mathrm{pH}$, among others. The act of sampling in the ocean takes into account the varying dynamics of this complex environment and in this research, we used the concept of environmental niche models to develop our models.

\subsection{Spatial Interpolation}

The basic process for creating a terrain map from the scientific and bathymetric data is to first generate a base map for each data parameter being collected. Then, determine a weighting schema that enables the maps to be brought together via linear combination while maximizing the contrast of the resulting terrain map. The most used interpolation techniques available are:

\subsubsection{Spline}

The Spline interpolation consists of estimating values through a series of piecewise polynomials (splines) to minimize the curvature of the surface. This surface must pass through the input points and is given by: 


$$
S(x, y)=T(x, y)+\sum_{j=1}^{N} \varepsilon_{j} R\left(r_{j}\right)
$$

where $j=1,2, \ldots, N, N$ is the number of datapoints, $\varepsilon_{i}$ are the coefficients of a system

of linear equations and $r_{j}$ is the distance from a certain point $(x, y)$ to the $j^{\text {th }}$ point. More information on the Spline method can be found in [Fra82], [MM88] and [ESRI18b].

\subsubsection{Inverse Distance Weighting (IDW)}

Inverse Distance Weighting (IDW) is an interpolation technique that takes into account the proximity of elements. In order to predict an unknown datapoint, this technique assumes that a certain datapoint influences the surroundings but this influence is decreased with distance. Points closer to a predicted location have higher weights than points farther away. These weights are determined by a function of inverse distance. To read more about this technique, see [Tun83] and [Wat85].

\subsubsection{Kriging}

Kriging is geostatistical technique that interpolate values for points in unmeasured locations not only from observed values at surrounding locations but by making assumptions about the underlying spatial relationships of points in the dataset. Most interpolation algorithms perform well for a dense and uniformly distributed dataset; however, kriging provided better results for the creation of the underlying terrain maps by compensating the influence of data clustering and by providing an error estimation that will help determine the trajectory of the robot in future missions. Kriging has been used for interpolation method of heavy-metal contamination in soil [HOBR14], air pollution maps for eastern China [RM15], concentration of chlorinated aliphatic hydrocarbons (CAHs) that contaminate shallow groundwater $\left[\mathrm{LLL}^{+} 15\right]$, and concentration of dissolved oxygen 
(DO) in wastewater treatment ponds [KIJT17]. Moreover, technique has been successfully used to create regional distribution maps for the interpolation of nonpoint sources of physical parameters, e.g., a high-precision underwater digital elevation model was developed in [ZXX15], concluding that kriging for terrain-aided navigation is better than other traditional interpolation methods in terms of accuracy.

Based on the detailed description of how Kriging works in [ESRI18a]. Kriging is given by a weighted sum of the data:

$$
\hat{Z}\left(s_{0}\right)=\sum_{i=1}^{N} \varepsilon_{i} Z\left(s_{i}\right)
$$

where $Z\left(s_{i}\right)$ is the a measure value at an $i$ th location, $\varepsilon_{i}$ is an unknown weight for the $i$ th location, $s_{0}$ is a prediction location and $N$ is the number of measure values.

In order to find the appropriate weight for computing this interpolation, it is necessary to evaluate the spatial arrangements of the dataset and depending on the fitted model of these measure points, predictions will be made. Two important steps for making these predictions are:

1. calculates the semivariograms and covariance functions

2. estimate the spatial autocorrelation values of the dataset

3. predict the unknown values for unmeasured locations

In order to calculate the semivariograms, we need to first calculate the difference squared between every pair of points over the measured locations:

$$
\hat{y}(\vec{h})=\frac{1}{2 N(\vec{h})} \sum_{\alpha=1}^{N(h)}\left[s\left(\alpha_{i}\right)-s\left(\alpha_{i}+\vec{h}\right)\right]^{2}
$$

where $h$ is the distance between pairs of datapoints (also known as lag), $N(\vec{h})$ is the number of pairs that are separated by $h, s$ is a datapoint value at location $i$. 
The semivariogram is an empirical graph of the averaged $\hat{y}(\vec{h})$ values on the y-axis and the lags $\vec{h}$ on the x-axis:

Different semivariograms for the datasets collected are shown in Figure 6.26 for the missions in Santa Catalina Island on July 13th and 14th 2016 and in Figure ?? for some of the missions at Lake Nighthorse on May 2nd 2018.

Following the calculation of the semivariograms, a model needs to be fitted from the points in the empirical semivariogram. This model is a continuous function or curve. The most used functions for kriging interpolation are: circular, spherical, exponential, gaussian and linear. Depending on the model selected, predicted locations might not form a smooth surface and interpolation can yield high estimation errors.

In the next figures, we can see the standard errors in prediction for the two missions whose predicted maps are shown in Figures 3.19 and 3.21.

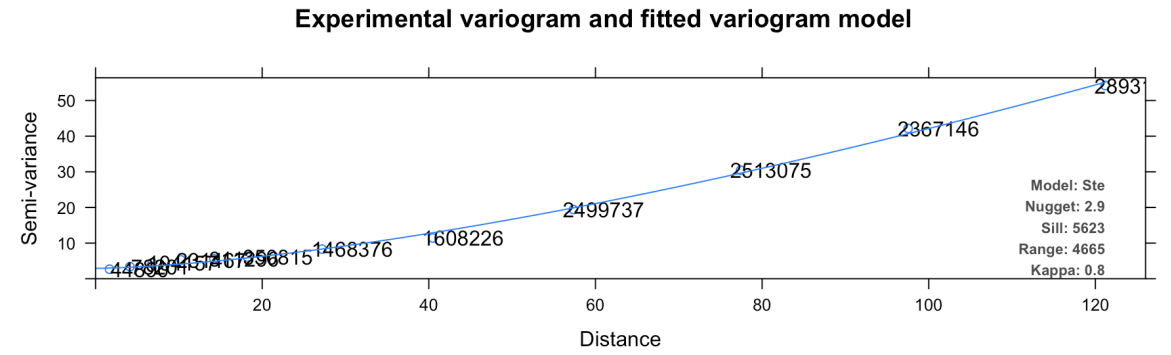

Figure 3.1: Semivariograms of the depth $(\mathrm{m})$ parameter generated in the datasets collected in Santa Catalina Island on July 13th 2016. 


\section{Experimental variogram and fitted variogram model}

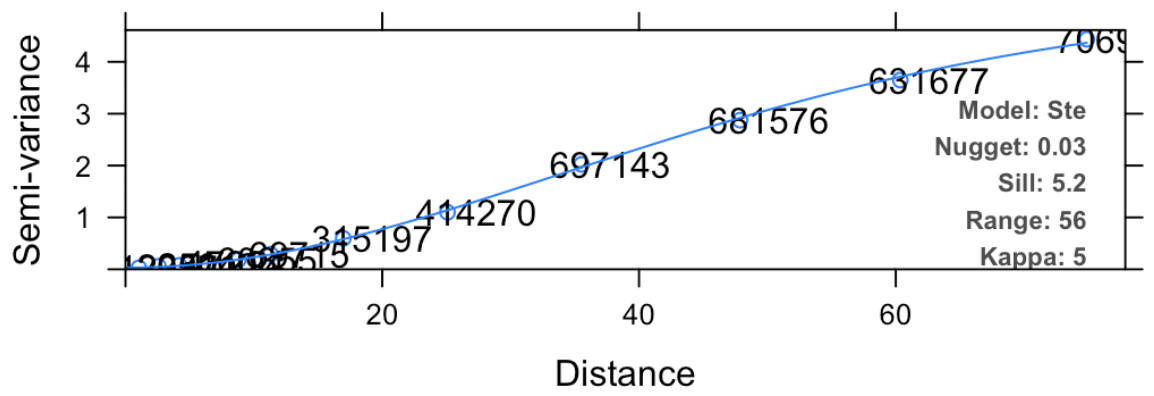

Figure 3.2: Semivariograms of the depth $(\mathrm{m})$ parameter generated in the datasets collected in Santa Catalina Island on July 14th 2016.

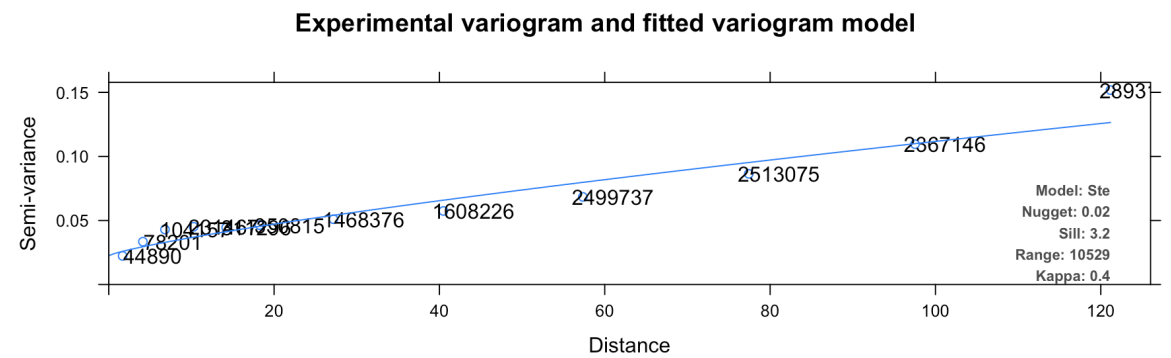

Figure 3.3: Semivariograms of the temperature $\left({ }^{\circ} \mathrm{C}\right)$ parameter generated in the datasets collected in Santa Catalina Island on July 13th 2016.

\section{Experimental variogram and fitted variogram model}

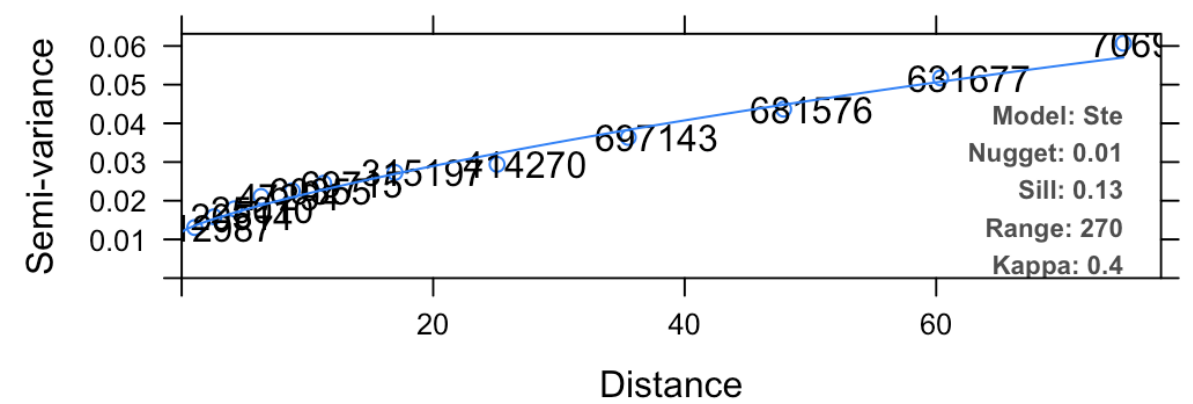

Figure 3.4: Semivariograms of the temperature $\left({ }^{\circ} \mathrm{C}\right)$ parameter generated in the datasets collected in Santa Catalina Island on July 14th 2016. 


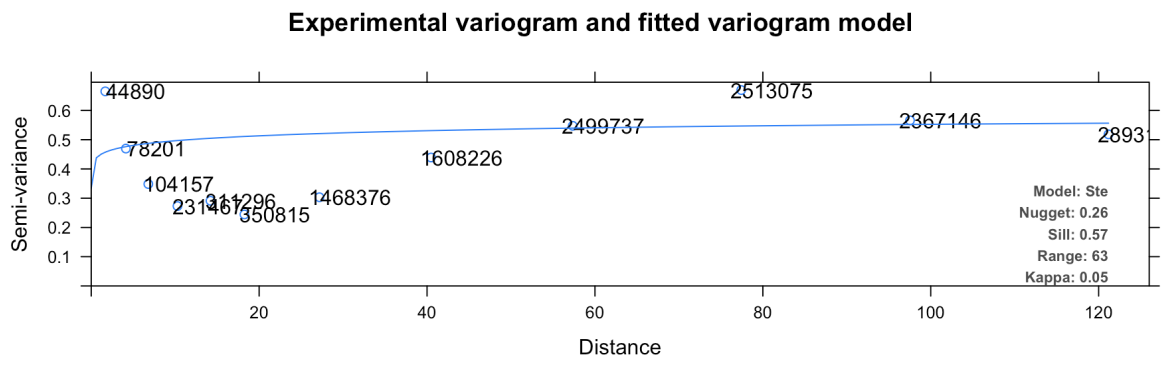

Figure 3.5: Semivariograms of the salinity (ppt) parameter generated in the datasets collected in Santa Catalina Island on July 13th 2016.

\section{Experimental variogram and fitted variogram model}

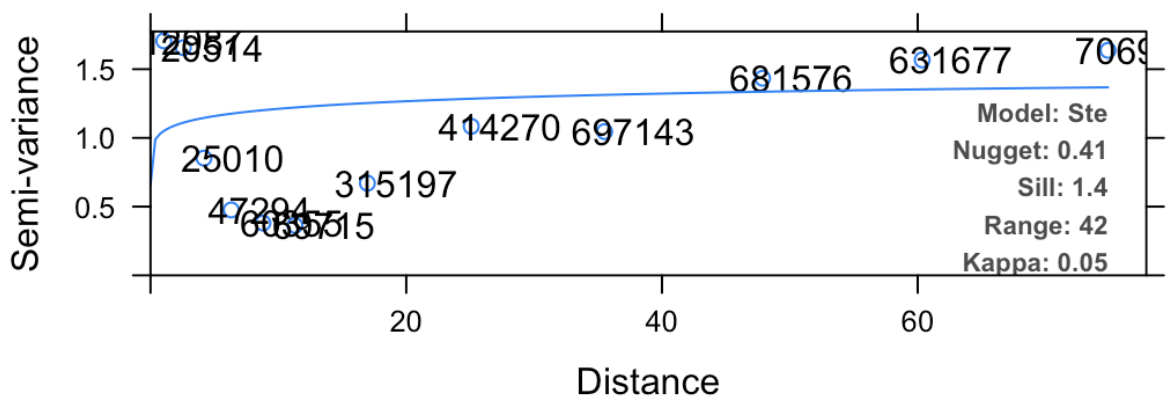

Figure 3.6: Semivariograms of the salinity (ppt) parameter generated in the datasets collected in Santa Catalina Island on July 14th 2016.

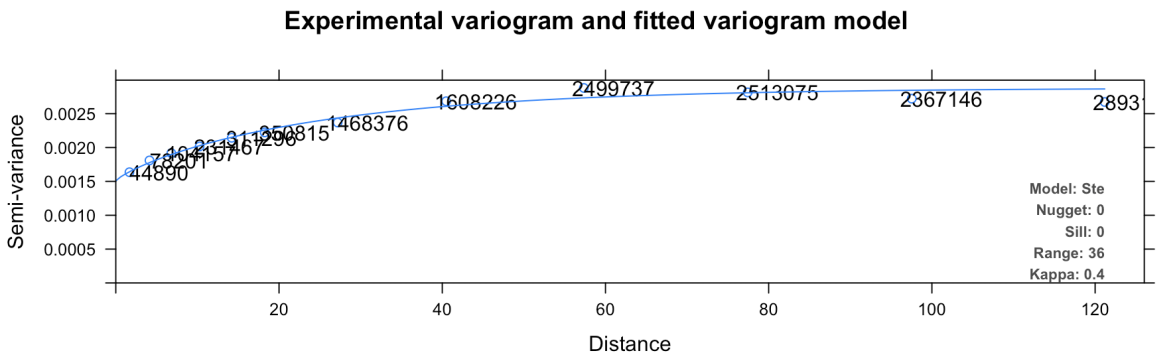

Figure 3.7: Semivariograms of the $\mathrm{pH}$ parameter generated in the datasets collected in Santa Catalina Island on July 13th 2016. 


\section{Experimental variogram and fitted variogram model}

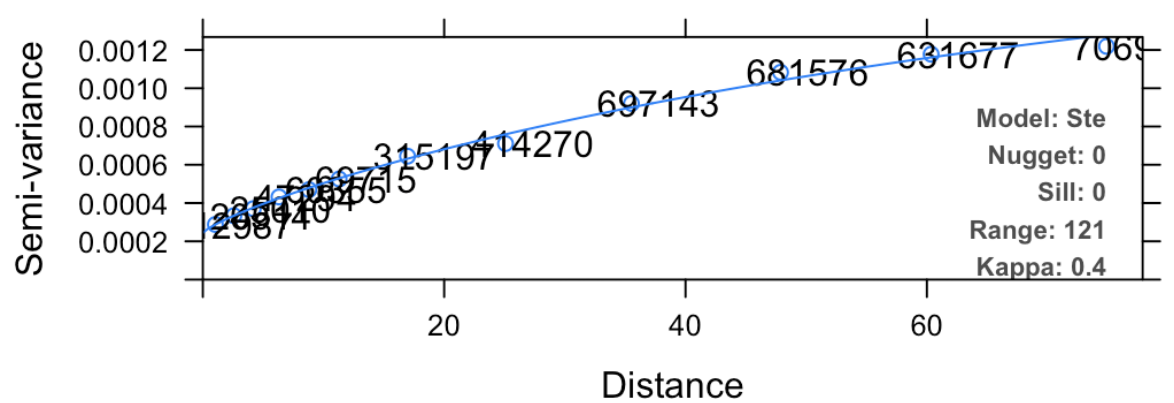

Figure 3.8: Semivariograms of the $\mathrm{pH}$ parameter generated in the datasets collected in Santa Catalina Island on July 14th 2016.

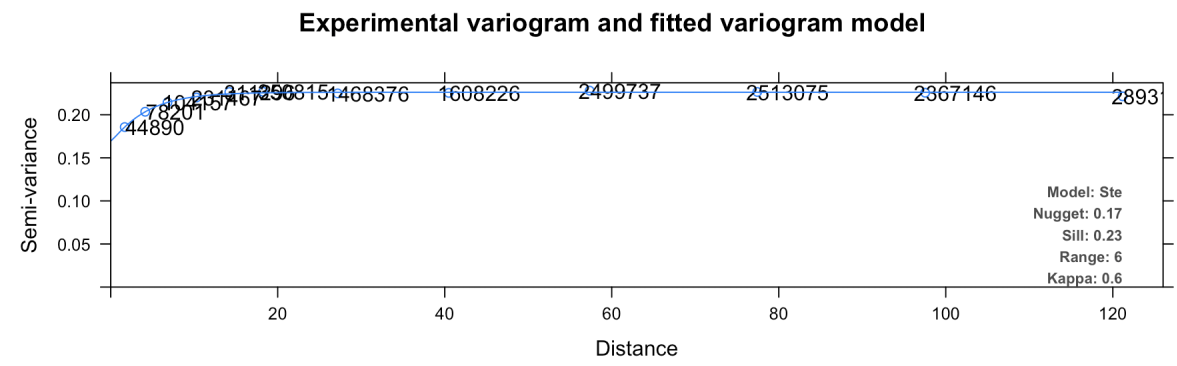

Figure 3.9: Semivariograms of the turbidity (NTU) parameter generated in the datasets collected in Santa Catalina Island on July 13th 2016.

\section{Experimental variogram and fitted variogram model}

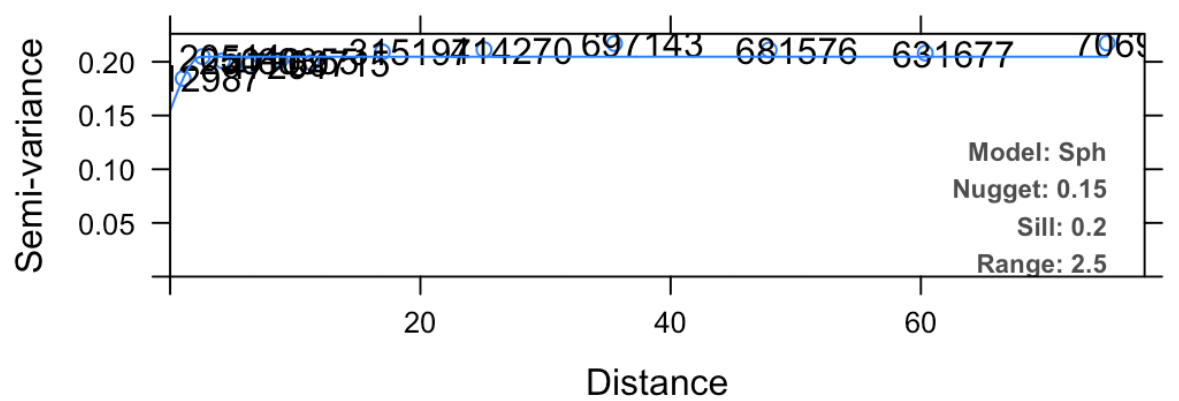

Figure 3.10: Semivariograms of the turbidity (NTU) parameter generated in the datasets collected in Santa Catalina Island on July 14th 2016. 


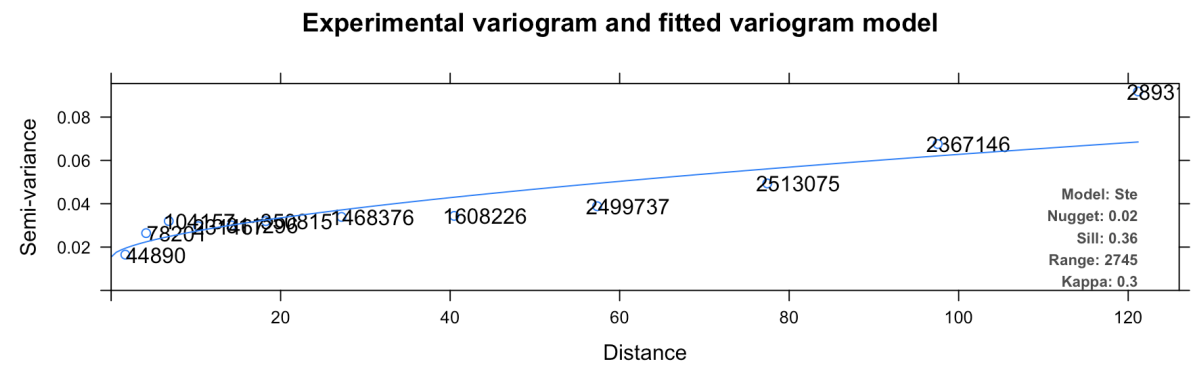

Figure 3.11: Semivariograms of the dissolved oxygen $(\mathrm{mg} / \mathrm{L})$ parameter generated in the datasets collected in Santa Catalina Island on July 13th 2016.

\section{Experimental variogram and fitted variogram model}

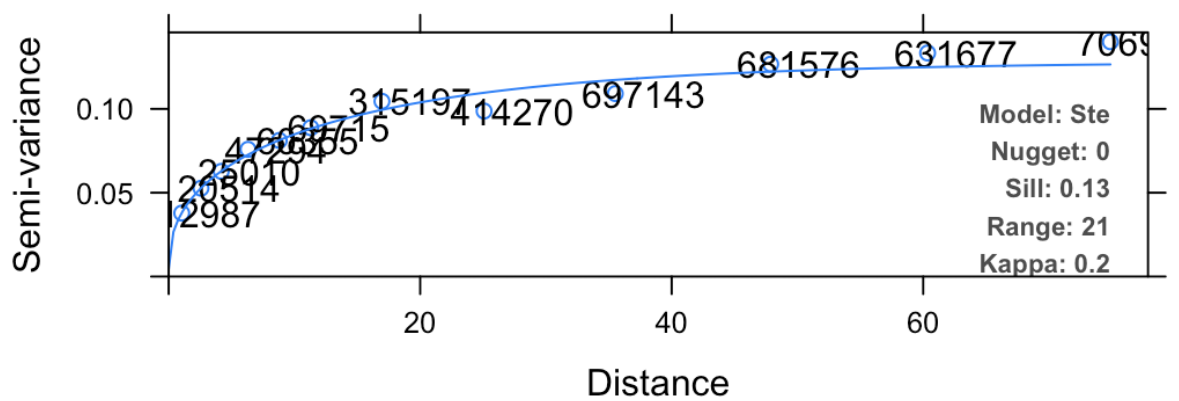

Figure 3.12: Semivariograms of the dissolved oxygen $(\mathrm{mg} / \mathrm{L})$ parameter generated in the datasets collected in Santa Catalina Island on July 14th 2016.

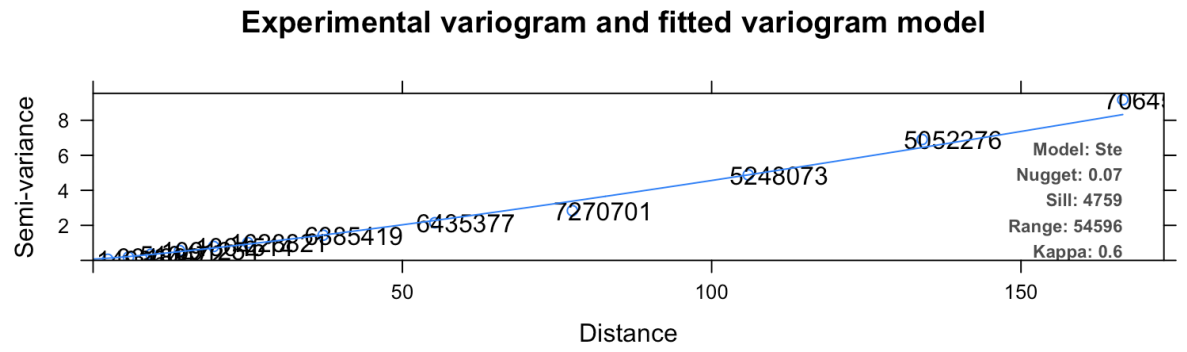

Figure 3.13: Semivariograms of the depth $(\mathrm{m})$ parameter generated in the datasets collected at Lake Nighthorse on May 2nd 2018. 
Experimental variogram and fitted variogram model

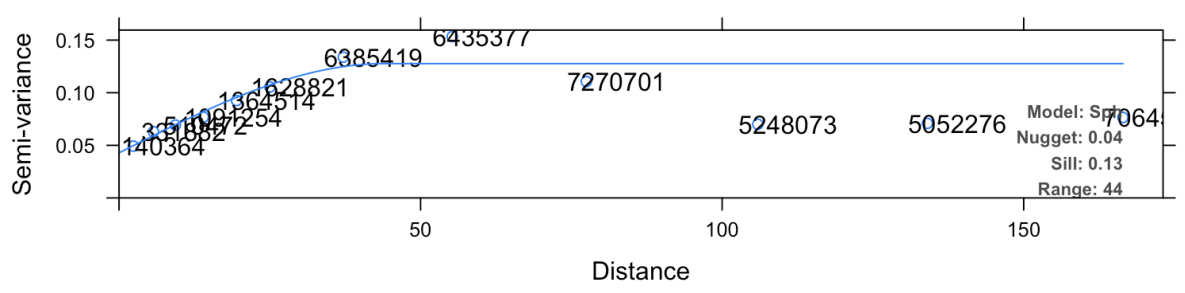

Figure 3.14: Semivariograms of the temperature $\left({ }^{\circ} \mathrm{C}\right)$ parameter generated in the datasets collected at Lake Nighthorse on May 2nd 2018.

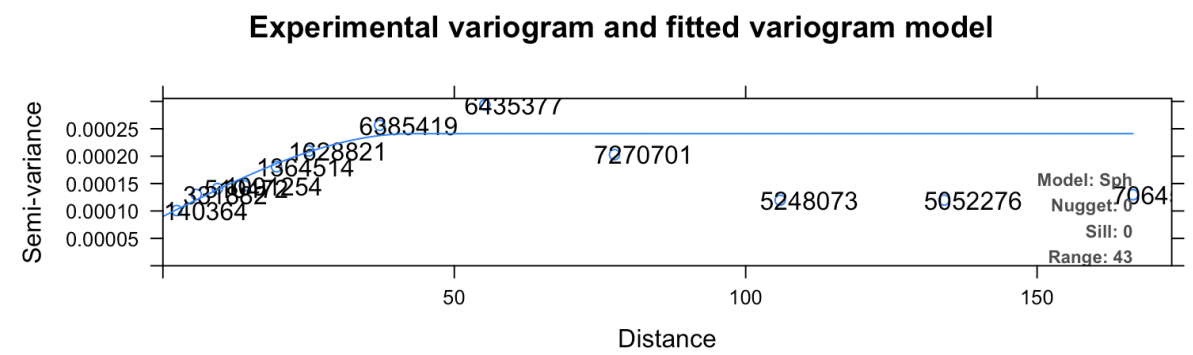

Figure 3.15: Semivariograms of the salinity (ppt) parameter generated in the datasets collected at Lake Nighthorse on May 2nd 2018.

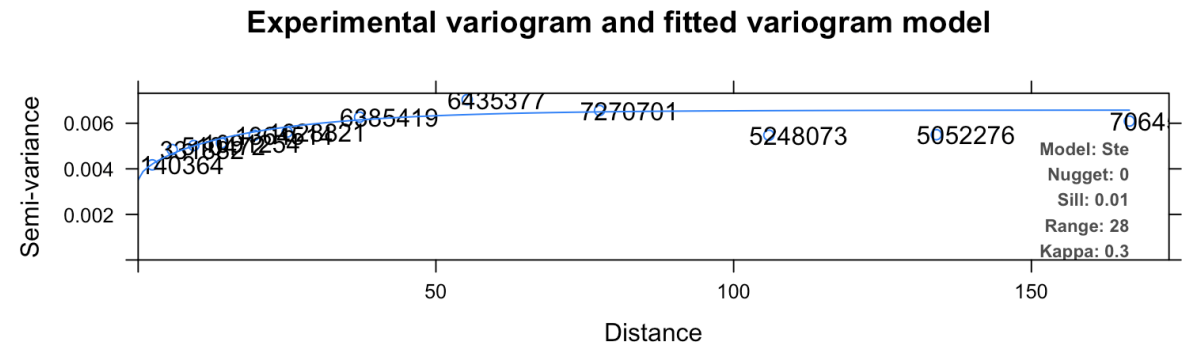

Figure 3.16: Semivariograms of the $\mathrm{pH}$ parameter generated in the datasets collected at Lake Nighthorse on May 2nd 2018. 


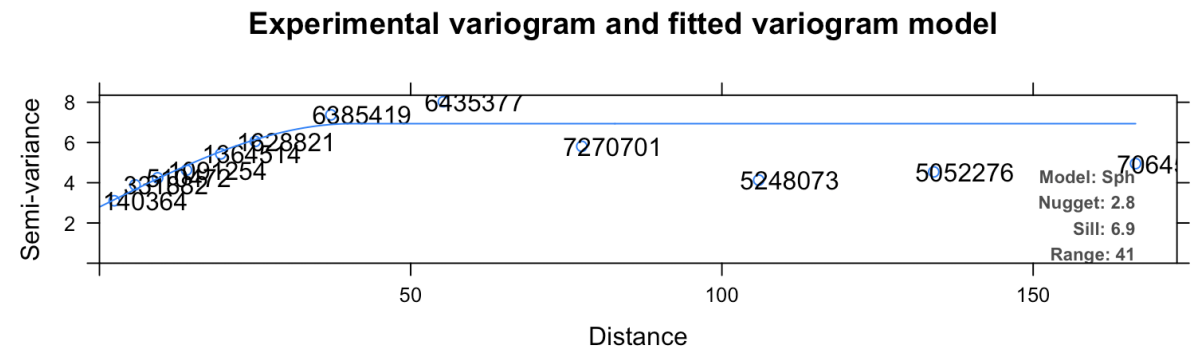

Figure 3.17: Semivariograms of the turbidity (NTU) parameter generated in the datasets collected at Lake Nighthorse on May 2nd 2018.

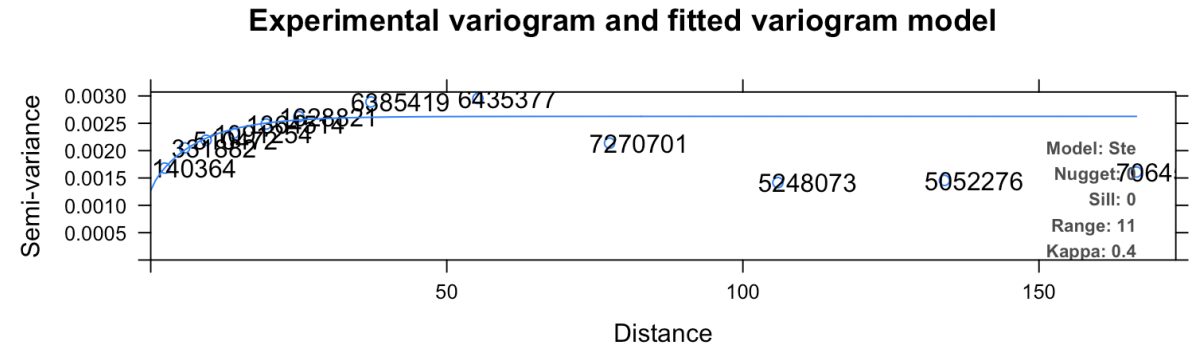

Figure 3.18: Semivariograms of the dissolved oxygen $(\mathrm{mg} / \mathrm{L})$ parameter generated in the datasets collected at Lake Nighthorse on May 2nd 2018. 
Kriging prediction

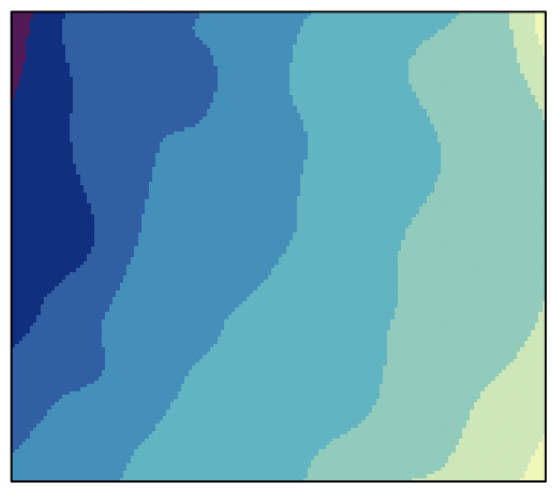

(a)

Kriging prediction

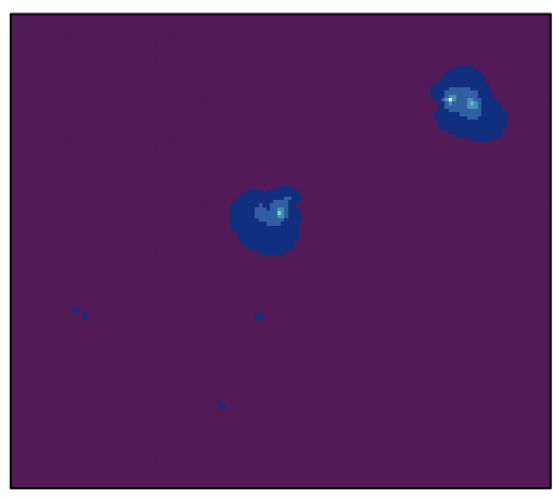

(c)

Kriging prediction

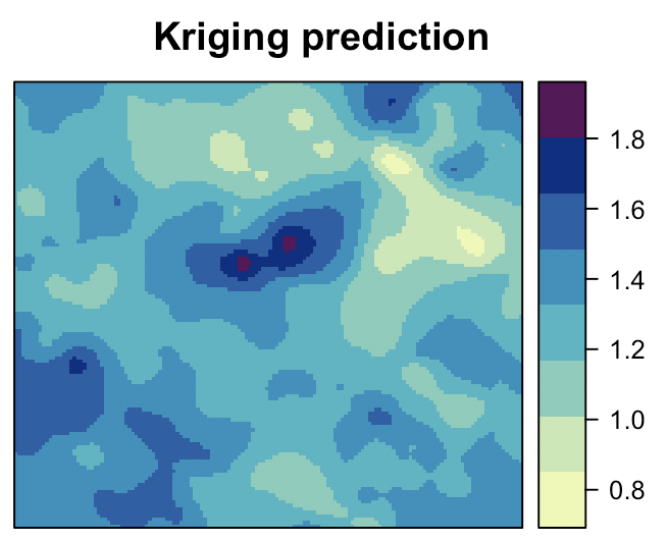

(e)
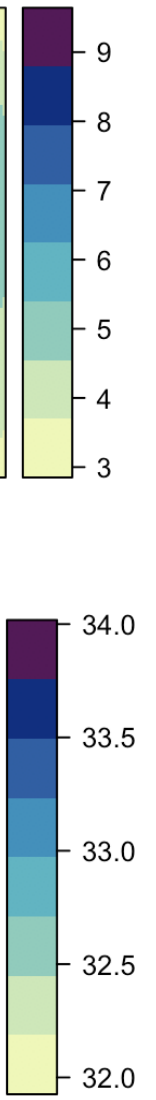

32.0

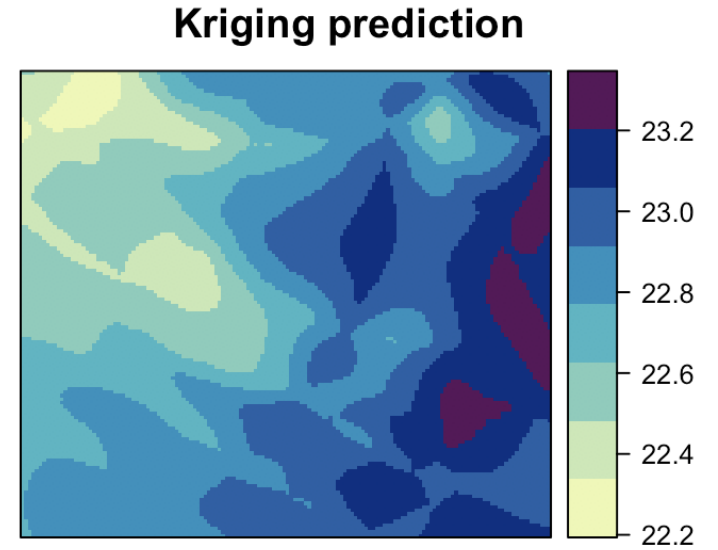

(b)

Kriging prediction

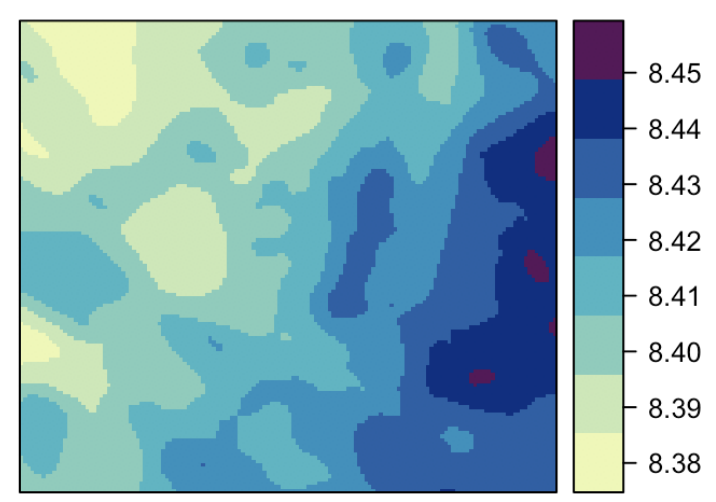

(d)

Kriging prediction

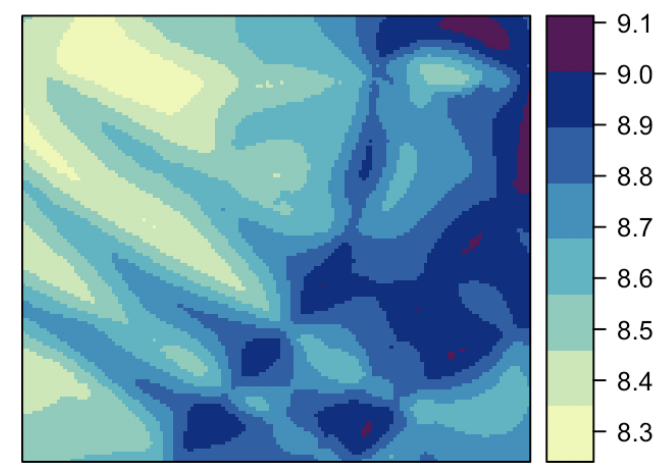

(f)

Figure 3.19: Predictions of values for the water parameters depth (a), temperature (b), salinity (c), $\mathrm{pH}(\mathrm{d})$, turbidity (e) and dissolved oxygen (f) generated through Kriging in the datasets collected in Santa Catalina Island on July 13th 2016. 
Kriging prediction

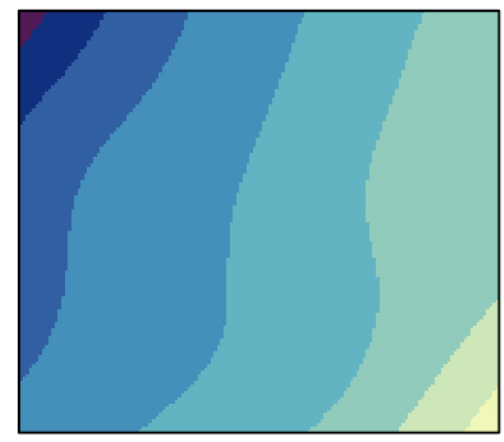

(a)

Kriging prediction
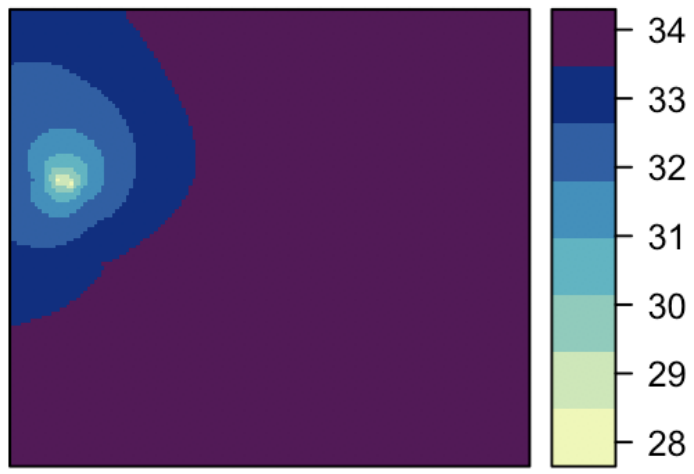

(c)

Kriging prediction

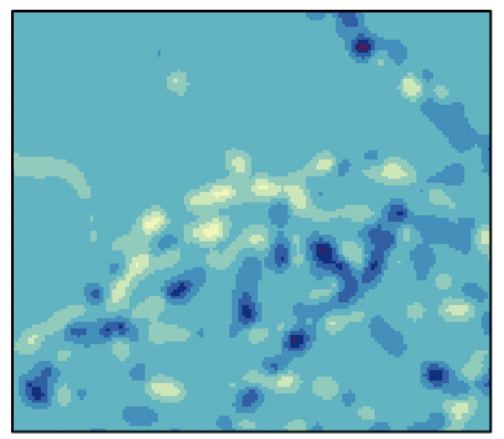

(e)
Kriging prediction

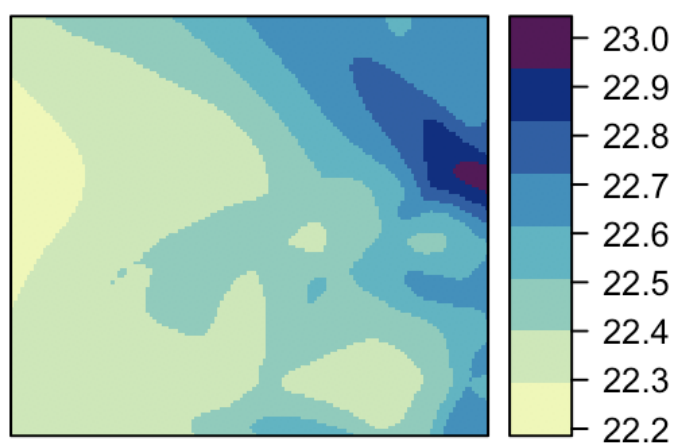

(b)

\section{Kriging prediction}

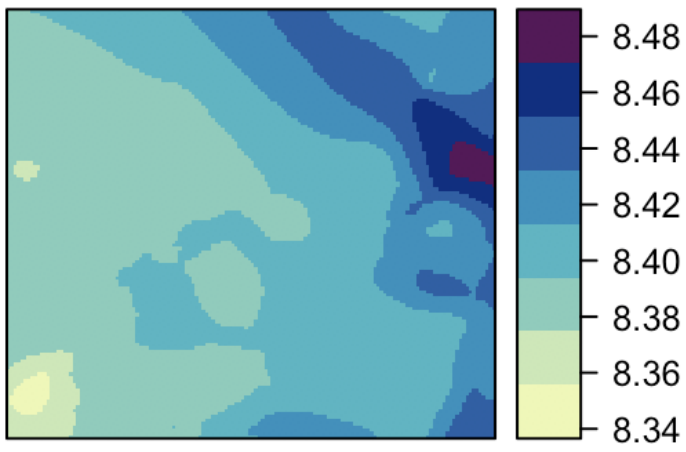

(d)

Kriging prediction

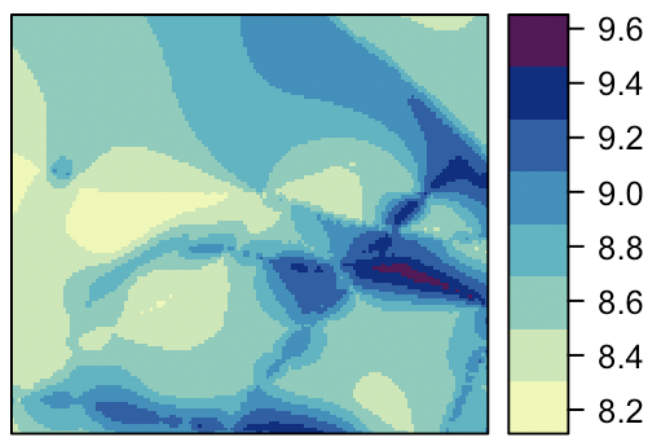

(f)

Figure 3.20: Predictions of values for the water parameters depth (a), temperature (b), salinity (c), pH (d), turbidity (e) and dissolved oxygen (f) generated through Kriging in the datasets collected in Santa Catalina Island on July 14th 2016. 
Kriging prediction
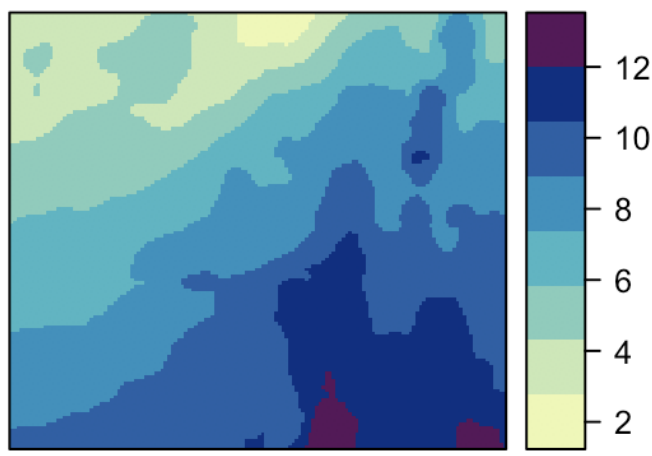

(a)

Kriging prediction
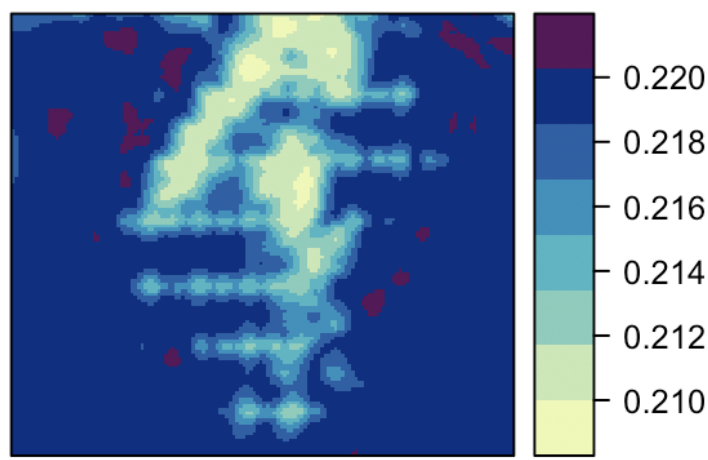

(c)

Kriging prediction
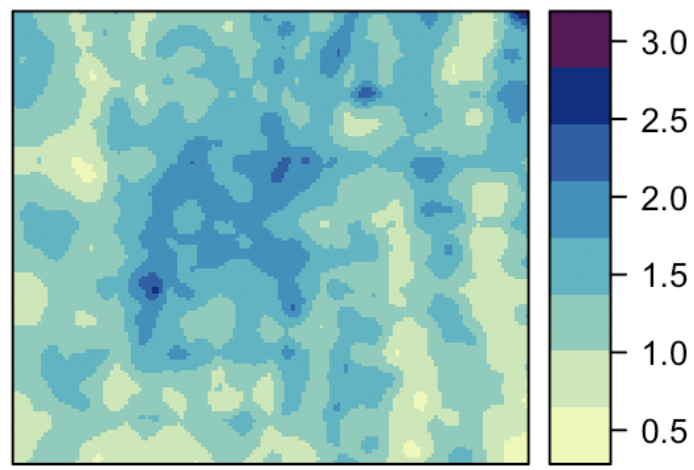

(f)
Kriging prediction

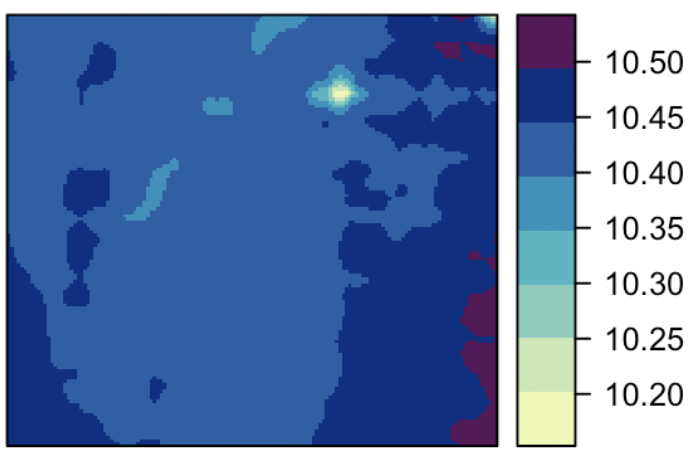

(b)

Kriging prediction

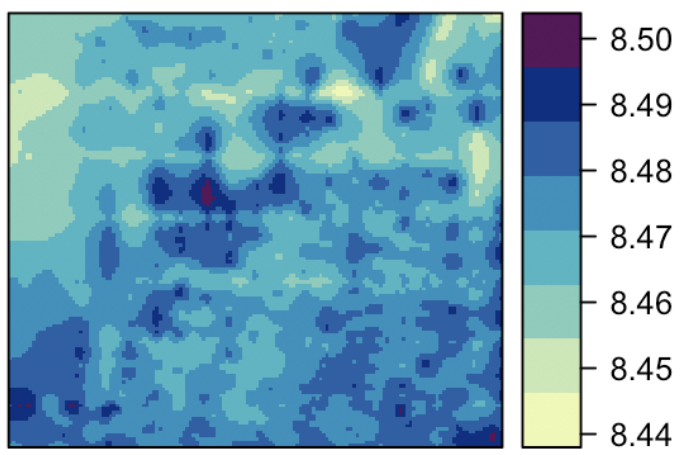

(d)

\section{Kriging prediction}

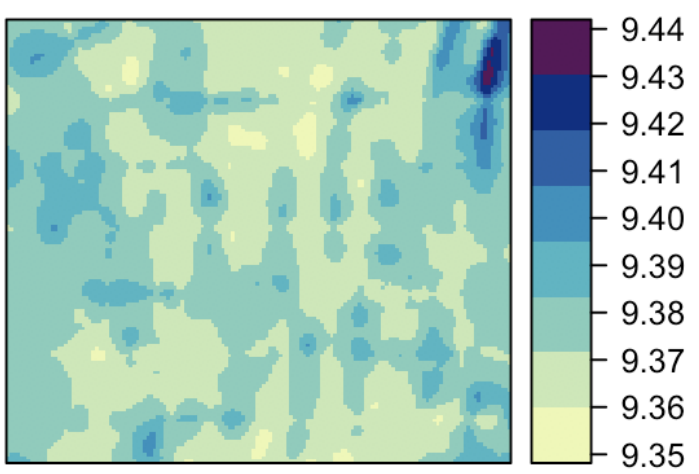

(g)

Figure 3.21: Predictions of values for the water parameters depth (a), temperature (b), salinity (c), $\mathrm{pH}(\mathrm{d})$, turbidity (e) and dissolved oxygen (f) generated through Kriging in the datasets collected at Lake Nighthorse on May 2nd 2018. 


\subsection{Informative Path Planning for Optimal Sampling}

In this subsection, we are interested in using the errors in prediction derived from the preprocessing of data described in the beginning of this chapter to formulate a path planning problem given the constraints of the dynamic behavior of the marine environment. The policies generated in here can be applied by simple drifters (floating aquatic robots) that are equipped with a downward-facing monocular camera, an inertial measurement unit (IMU), a GPS sensor, a WiFi communication module, and a Raspberry PI computing unit, making it suitable for the deployment in a marine environment for long time periods, ranging from days to weeks. These drifters can float on the water carried by currents, waves, and wind and collect data on various water parameters, e.g., temperature, salinity, turbidity, and $\mathrm{pH}$. Some more advanced drifters, equipped with actuators, can also move vertically in the water controlling the buoyancy based on the concept of controlled drift [SSS $\left.{ }^{+} 11 \mathrm{~b}, \mathrm{SH} 14\right]$ and know its initial condition. These floating robots are also known as profiling floats.

We use the datasets collected at the Big Fisherman's Cove, in Santa Catalina Island, CA, USA, on July 13th 2016 and at the Lake Nighthorse, Durango, CO, USA, on May 2nd 2018. These oceanic and freshwater data present the variations in spatial and temporal dimensions which are important for obtaining the persistent behavior analysis that can be further applied to other bodies of water.

Predictive models in path planning in the ocean have been investigated by $\left[\mathrm{CMN}^{+} 92\right.$, KSBB07, GAO05, WD08, $\mathrm{PPP}^{+}$07, ACO04], where the authors had the objective of solving path planning optimization problems. In the ocean studies, one of the biggest motivation is to minimize energy consumption and to extend a vehicle's deployment time. To add to this, complex current structures experienced in a coastal region can vary significantly with time and location, making subsurface navigation difficult. In [CMN $\left.{ }^{+} 92\right]-[\mathrm{ACO} 04]$, 
the authors addressed the problem of path planning for AUVs given the complex, timedependent, variable ocean.

In $\left[\mathrm{SCL}^{+} 10 \mathrm{a}\right]$, path planning and trajectory design of AUVs called gliders was proposed by analyzing dynamic features by the use of a glider in the Southern California coastal ocean. The critical assumption of their work is the ability of the glider to navigate to a given waypoint accurately. They did not consider the motion uncertainty of an AUV. Nevertheless, authors were able to propagate the errors due to the uncertainty of the motion of AUVs in $\left[\mathrm{SKC}^{+} 10\right]$. The motion of different underwater vehicles were modeled as Gaussian Processes in [OLCJ14, MLS17] for path planning as well as sensing and predicting the underlying phenomena in persistent ocean monitoring. The long-term trajectories of the underwater vehicles were also applied to persistent monitoring [SSS $\left.{ }^{+} 11 \mathrm{~b}, \mathrm{MLS} 16\right]$ of small or large aquatic environments. Marine robots are usually designed and deployed for environmental monitoring [FBLS03], collecting large-area ocean data [SSS ${ }^{+} 11 \mathrm{~b}$ ], and tracing chemical plumes [FPL05, HTH15]. ASVs have been used to monitor invasive fish species with radio transmitters across a marine environment [TBSI10]. Also, the sensorrich autonomous underwater vehicles (AUVs) such as marine gliders are popular platforms $\left[\mathrm{LPD}^{+} 10, \mathrm{MLW}^{+} 16, \mathrm{PZL} 08 \mathrm{c}\right]$. These platforms have high sensing and actuation capabilities which leads to a computation intensive planning and the use of a wealth of resources. The planning method that navigates robots to achieve and maximize information gain through sampling the environment is called informative planning [BKS10, BKS13]. Here, we would like to use the errors in prediction of the maps generated for each water parameter and develop policies for the AUV to execute missions in the same area of interest decreasing its path and maximizing its gain.

We consider a 2D marine environment as a workspace denoted as $\mathscr{W}=\mathbb{R}^{2}$. The environment is discretized into a $2 \mathrm{D}$ grid map. Let $\mathscr{O}$ be the land region of the environment where the AUV has already collected substantially many observations during its first mis- 
sion. The free water space of the marine environment is denoted as $E$ excluding the land region $\mathscr{O}$. Each equal-size grid tile in the grid map is called a cell.

The cell is represented as $z=\left(x_{t}, y_{t}\right)$ in which $x_{t}, y_{t} \in \mathbb{R}$ denote longitude, latitude, and water parameter of the center of a cell $z$. We model the AUV $\mathscr{D}$ as a point robot without considering its orientation. The state space of the AUV $\mathscr{D}$ is denoted by $X=E$ and it includes all the navigable locations in the environment $E$. A state of the AUV in the state space is indexed by a cell index $z \in\{1, \ldots, N\}$ where $N$ represents the total number of cells in $X$. Let $Z=\{1, \ldots, \mathrm{N}\}$ denote the set of all cells in the state space. Let $x_{I}$ be an initial state or location of the AUV on the water surface. The action set of the AUV among different layers $U=\{\uparrow, \leftarrow, \rightarrow, \downarrow\}$ which means the AUV can move up $(\uparrow),(\leftarrow),(\rightarrow)$ and down $(\downarrow)$, but not idle at a particular location (we assume that the vehicle is constantly moving).

From a non-boundary cell $z$ of $E$, the AUV $\mathscr{D}$ moves either towards one of the directions of N, NE, E, SE, S, SW, W, NW. While collecting data in a marine environment, it is essential to find the locations where the AUV can reach in the long run from its initial deployment location on the water surface. This information will help us deploy the AUV in different initial locations to increase the reward and obtain as much as data possible of the environment. Let the set of all reachable cell locations from the initial deployment location $x_{I}$ over a long time period be $R \subset Z$. In this context, we formulate our planning problem as follows:

Problem 1. Optimal Policies for an AUV Given an underwater environment E with the flow and an initial deployment location of an AUV $x_{I}$, find the optimal policies. The world is freespaces (0) or obstacles (1). The robot can move in 4 directions (given by $U$ ). A reward function gives the regions of higher errors in prediction higher reward. All other freespaces have a small penalty, and obstacles might occur or not and have a large negative reward. 
A value iteration method is used to learn an optimal policy find a policy for the decision maker: a function $\pi$ that specifies the action $\pi(s)$ that the decision maker will choose when in state $x_{i}$. We also consider a framework of a stochastic environment with fullyobservable states which is known as a Markov Decision Process (MDP) [TBF05]. The methodology is based on the implementation found in [Bec15].

In an MDP, the policy $\pi: \mathscr{X} \rightarrow U$ maps from each state $x \in \mathscr{X}$ to a possible action $u \in U$ when the state is observable. The goal of the MDP framework is to identify the policy $\pi$ that maximizes the potential reward. Hence, a reward function of the state and the action is denoted as $r$. For instance, the reward function for reaching the goal state $x_{G}$ can be initialized as follows:

$$
r(x, u)= \begin{cases}100, & \text { if } u \text { leads to } x_{G} \\ -1, & \text { otherwise }\end{cases}
$$

The reward function is given my the errors in the prediction originated from kriging interpolation in Section 3.6.3. For each predictive map, an error in prediction for each $z=\left(x_{t}, y_{t}, l_{t}\right)$ is given by the square root of mean-square-error (root-mean-square-error RMSE). One may choose to use mean absolute error (MAE) over RMSE, but according to [CD14], RMSE satisfies the triangle inequality requirement for a distance metric. Moreover, the authors discussed some circumstances where RMSE is more beneficial than MAE. To visualize the errors in prediction for each water parameter, see Fig. 3.22 for Santa Catalina Island on July 14th and Fig. 3.23 for the Lake Nighthorse on May 2nd 2018. 
Kriging standard error
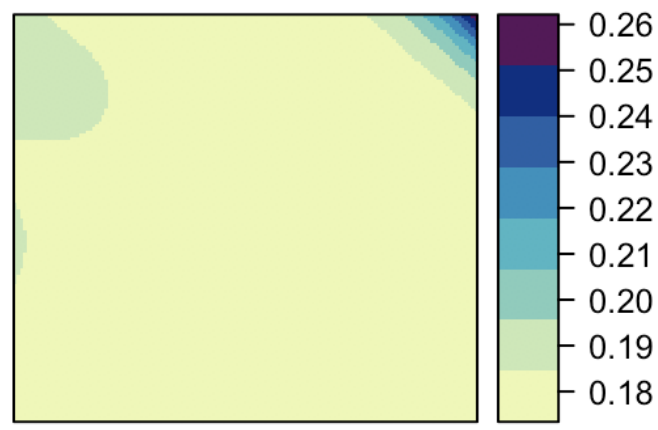

(a)

\section{Kriging standard error}
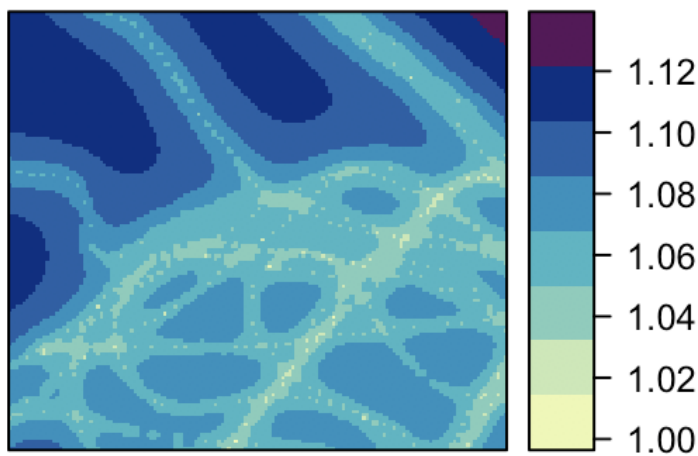

(c)
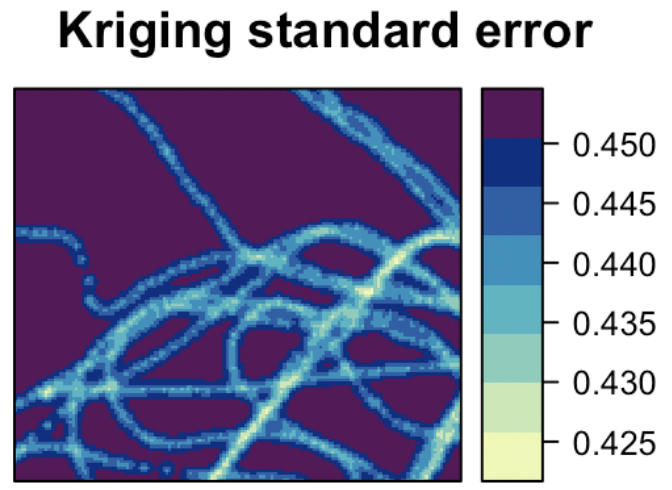

(e)
Kriging standard error

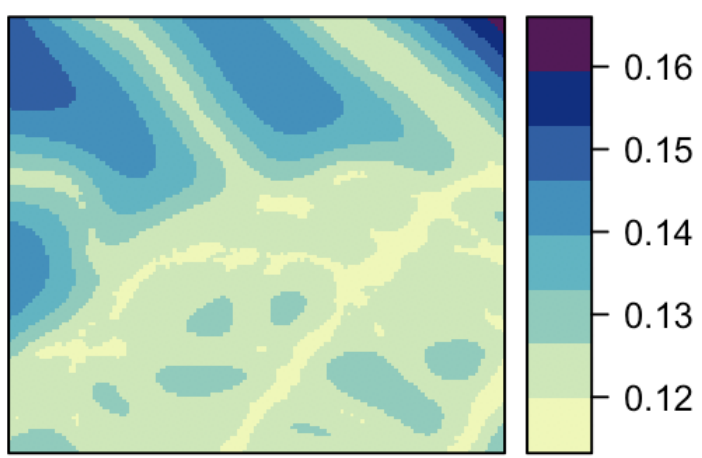

(b)

\section{Kriging standard error}

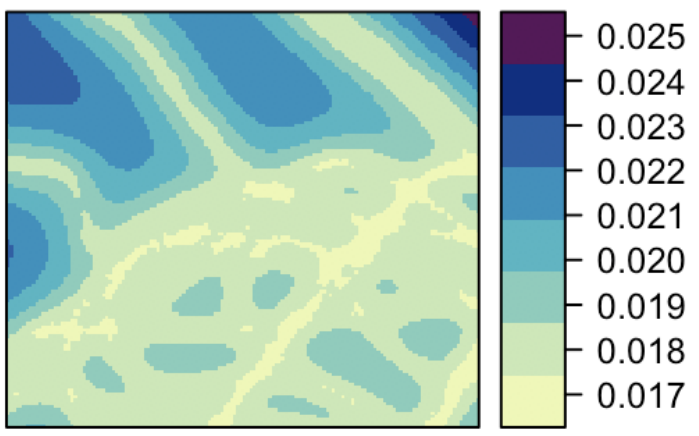

(d)

Kriging standard error

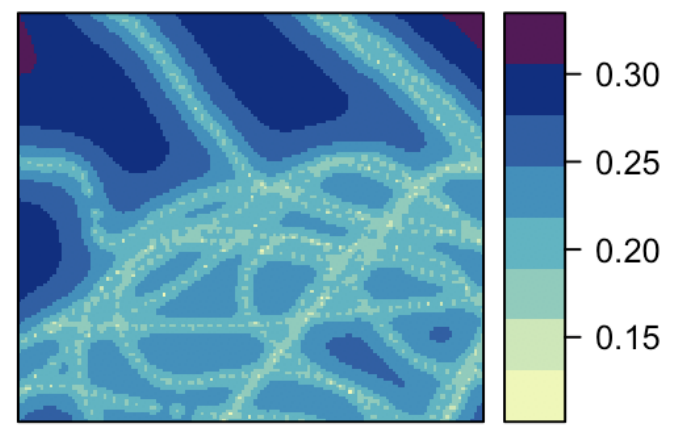

(f)

Figure 3.22: Errors in predictions for the water parameters depth (a), temperature (b), salinity (c), $\mathrm{pH}$ (d), turbidity (e) and dissolved oxygen (f) generated through Kriging and calculated using the square root of mean-square-error for the datasets collected in Santa Catalina Island on July 14th 2016. 
Kriging standard error

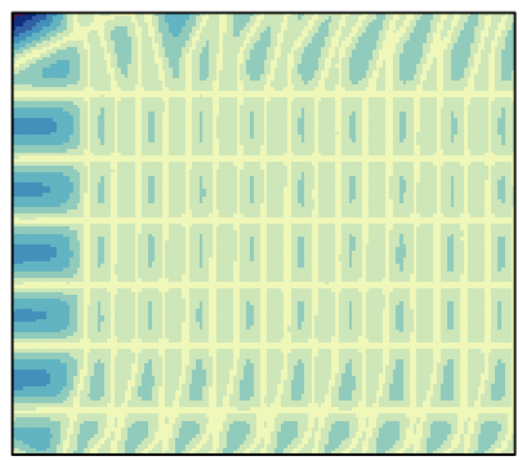

(a)

Kriging standard error
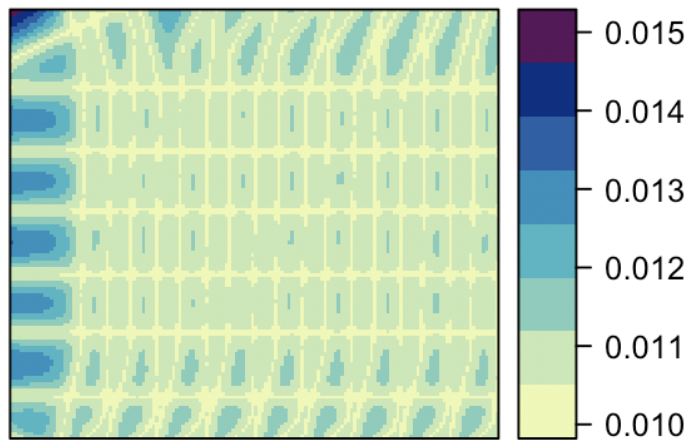

(c)

Kriging standard error

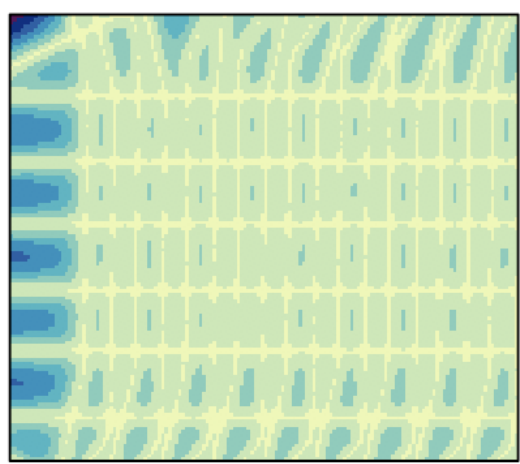

(e)
Kriging standard error

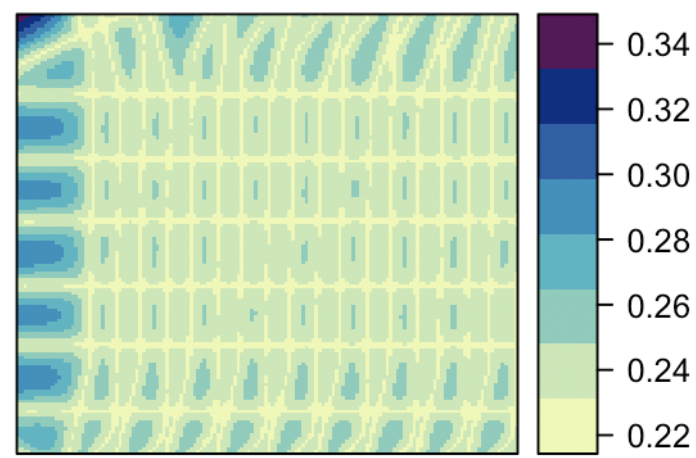

(b)

\section{Kriging standard error}
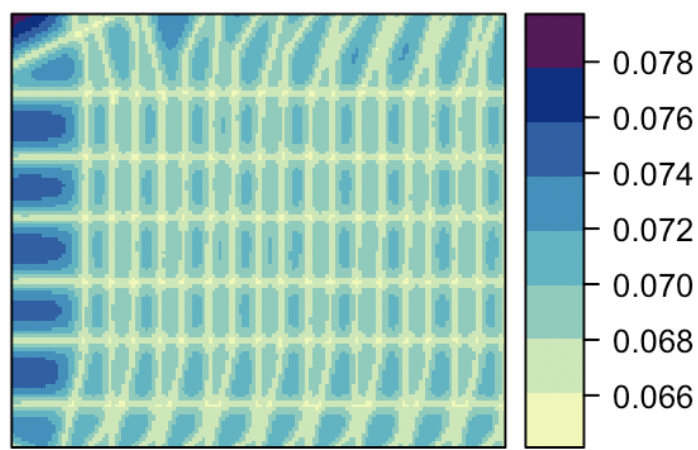

(d)

\section{Kriging standard error}
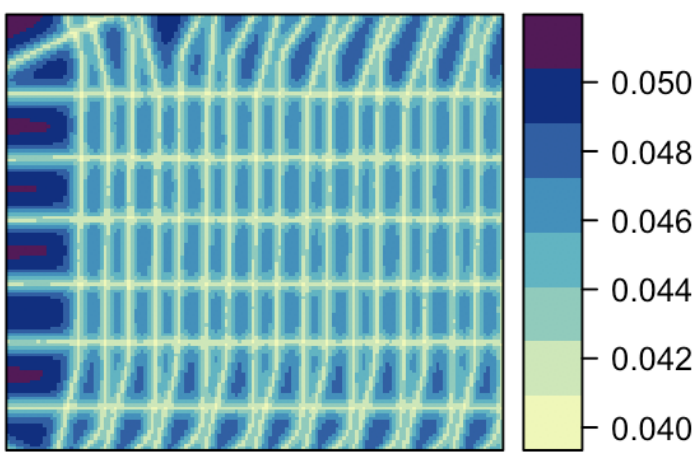

(f)

Figure 3.23: Errors in predictions for the water parameters depth (a), temperature (b), salinity (c), pH (d), turbidity (e) and concentration of dissolved oxygen (f) generated through Kriging interpolation and calculated using the square root of mean-square-error for the datasets collected during a mission at Lake Nighthorse, Durango, Colorado, USA, on May 2nd 2018. 


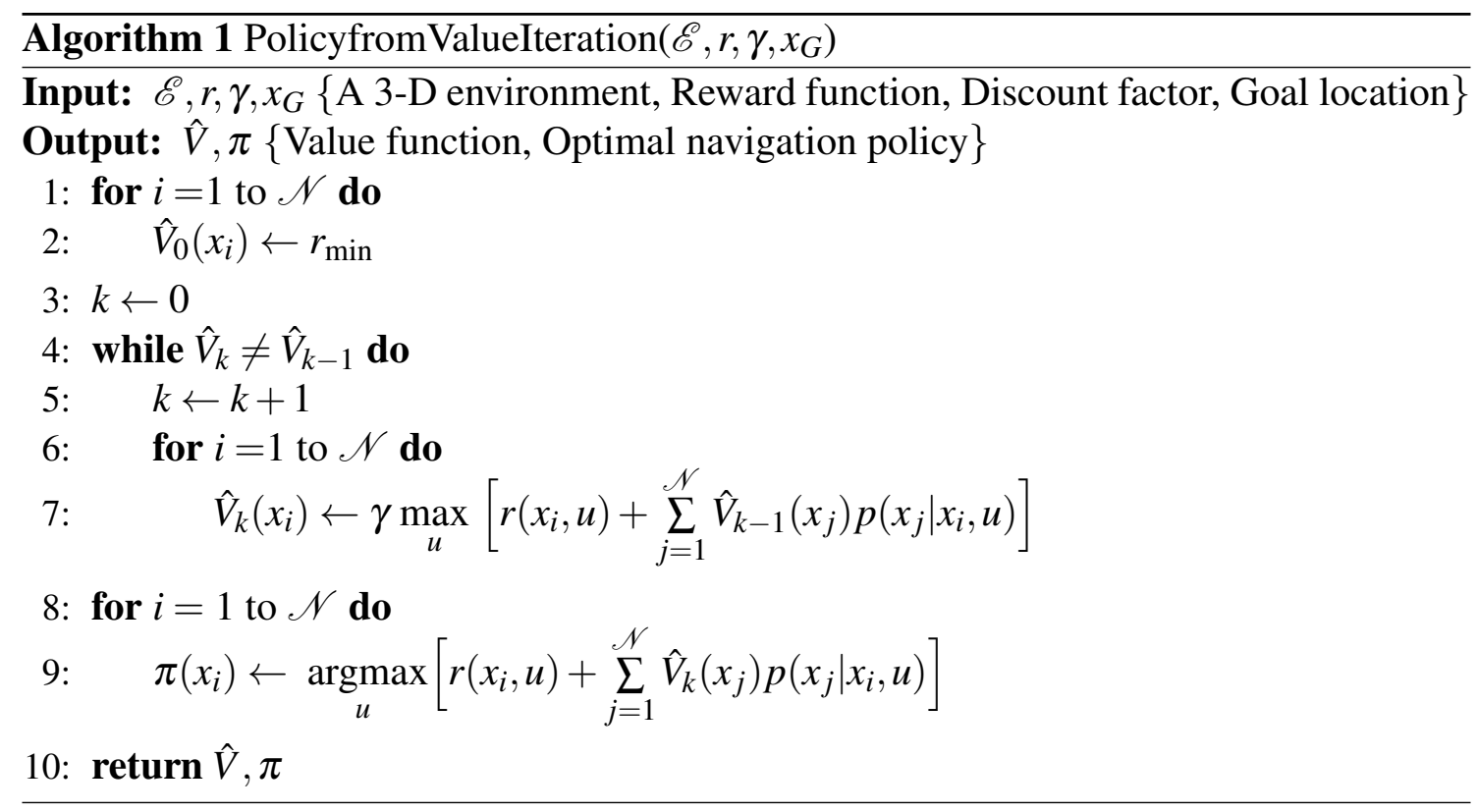

There is a value function associated with each policy, which is the measurement of the expected value of the policy. Let the value function be $\hat{V}$. Algorithm 1 initializes the value function $\hat{V}$ with $r_{\min }$, which represents the minimum possible immediate reward (lines 1-2). Then, it implements the recursive calculation of $\hat{V}$ using the value iteration method (lines 4-7). Once the value iteration converges after a number of iterations $k$, the resulting value function $\hat{V}_{k}$ that maximizes the expected value of the function, induces the optimal navigation policy. The factor $\gamma$ is the discount factor. The value iteration usually converges if $\gamma<1$, and in some special cases, even for $\gamma=1$. The final value function $\hat{V}_{k}$ after the convergence of the value iteration is the optimal value function. Thus, Algorithm 1 calculates the optimal navigation policy $\pi$ from the optimal value function maximizing the expected reward for reaching the goal location (lines 8-9). This navigation policy $\pi$ produces an optimal action from any location of the environment $\mathscr{E}$ to the given goal location $x_{G}$ as can be seen in Figure 3.24(a) for the case of the Big Fisherman's Cove in Santa Catalina Island when just bathymetric information is necessary; for instance to be used in regular terrain-based navigation approaches; Figure 3.24(b) for the 
Big Fisherman's Cove in Santa Catalina Island when water parameters and bathymetry information are required to be re-sampled on the first day and on the second day of deployment is shown in Figure 3.24(c). Figure 3.24(d) shows an optimal action from any location of the environment for the case of Lake Nighthorse in Durango, CO, USA.

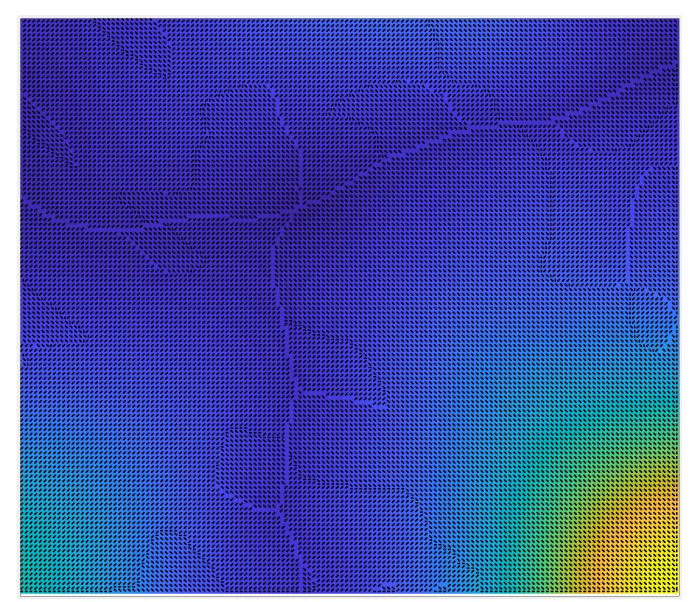

(a)

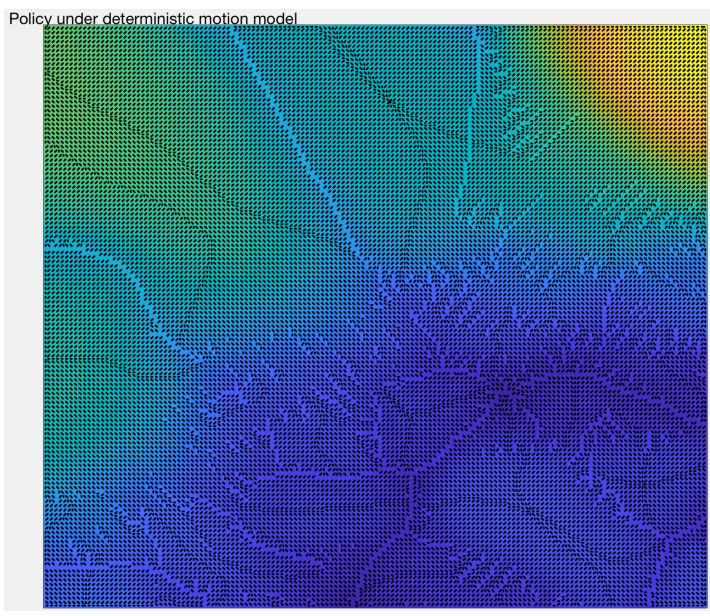

(c)

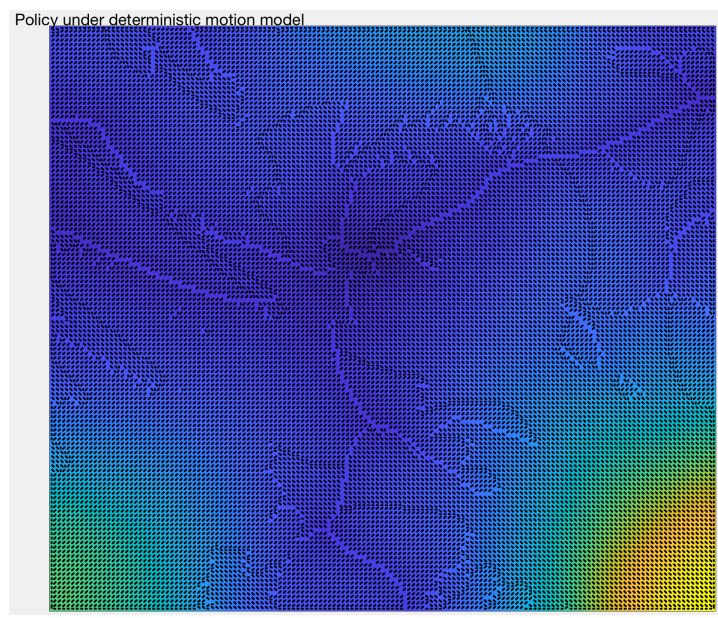

(b)

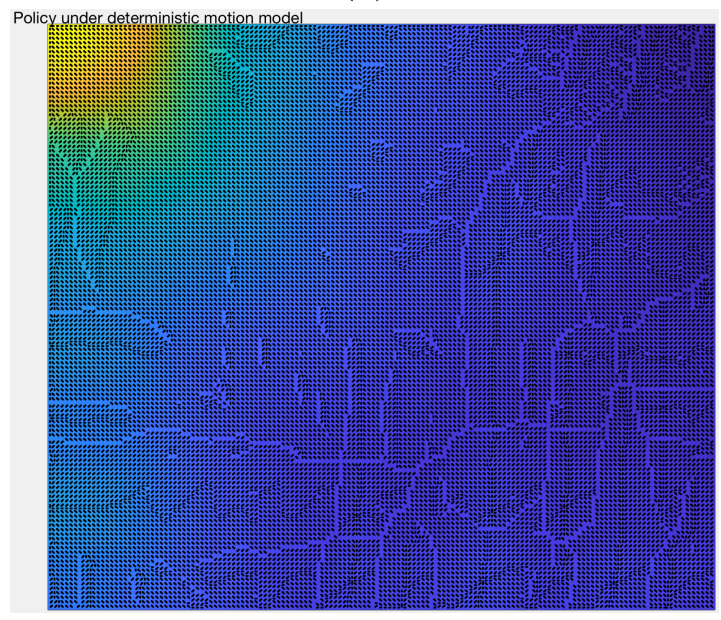

(d)

Figure 3.24: Simulation results of the long-term deployment policy for the informative sampling in (a) Santa Catalina Island, CA, USA for just bathymetry; (b) Santa Catalina Island, CA, USA for the combination of water parameters and bathymetry on the first day of deployment; (c) Santa Catalina Island, CA, USA for the combination of water parameters and bathymetry on the second day of deployment; (d) Lake Nighthorse, CO, USA for the combination of water parameters and bathymetry. 


\subsection{Conclusion}

In this chapter, different 2-D interpolation methodologies that are used in modeling were presented. Moreover, a kriging-based solution for the problem of finding the value of the unknown locations in the environment was explained and suggested as the pre-processing step in the creation of reference maps in localization of underwater robots. The semivariance of each water parameter and bathymetry was computed to measure the spatial dependence between any two observations as a function of the distance between them and the semivariograms (which shows how semivariance changes as the distance between observations changes) of different missions and deployments of the YSI Ecomapper AUV in two different bodies of water, the Big Fisherman's Cove in Santa Catalina Island, CA, and the Lake Nighthorse in Durango, CO, were analyzed. Kriging showed the best results for finding the values of unknown locations in the region of interest and may be viable to be used on-board of the vehicles during the missions. Furtheremore, a data-driven approach for informative sampling of autonomous underwater vehicles was examined and presented. An optimal navigation policy was presented in the 2-D marine environment that generates the best possible action in the simulated policy from any location of the environment to the goal location. This policy can be used to decrease the error in prediction in regions where a second mission is necessary considering the battery constraint and considering that there are no obstacles in the environment. The error in prediction obtained from the kriging interpolation technique was used as the reward function in this Markov Decision Process approach. 


\section{CHAPTER 4}

\section{MAP CREATION}

\subsection{Introduction}

In the last two chapters, we listed the parameters we collected for the present research, we described the locations and the dates and part of the day which the data was collected and presented the technique utilized for processing the data. In the end of chapter 3 , we had one map for each one of the water parameter (e.g., temperature, salinity, concentration of dissolved oxygen, turbidity, $\mathrm{pH}$ and chlorophyll) and the bathymetric map of each location. In this chapter, we will examine the main process of creating the reference maps to be used in the localization of autonomous underwater vehicles that uses the maps created in chapter 3 . Having the bathymetric map as a base and improving its utility for geophysical systems of localization using water parameters, it is necessary to compare different methodologies for finding how much each water parameter can contribute to increasing the utility of the reference map. Different techniques for finding the appropriate weights will be explored, such as sampling in the simplex, Dirichlet Distribution, Principal Component Analysis and a novel Global Correlation score will be presented and compared to the widely used Moran's I technique to measure the spatial autocorrelation of the maps. The contributions of this chapter lies in the process of creating a reference map using a linear combination of bathymetric information and water parameters

\subsection{Related Work}

In this section, the motivation for enhancing the utility of maps using bathymetric with water parameters will be presented, followed by a literature review on different methodologies for creating reference maps for localization. 


\subsubsection{Ecological Niche Models}

Navigation methods used nowadays are usually based on satellite information from the Global Positioning System (GPS) to keep estimation accuracy. Nevertheless, GPS signals are not able to penetrate water; therefore, autonomous underwater vehicles (AUVs) can only use GPS when they are at the surface of the aquatic environment. In consequence, most navigations systems for AUVs use some form of dead-reckoning together with several techniques to bound the error growth using external information (e.g., Geophysical Navigation, which is presented in Chapter 5). It is known that the ocean environment exhibits a naturally stochastic and aperiodic characteristic; however, it is possible to identify coherent structures that can be exploited. Examples of applications of such structures can be found in [PEWF08, FLCS12, LSF07] for facial recognition, city modeling [FZ03], novel view synthesis for 3D visualization [CMR10], and robotic localization tasks [SE12, $\mathrm{URO}^{+}$08]. This approach has been fairly used in marine environments due to the challenges in engineering infrastructure for large-scale field deployments; the spatio-temporally dynamics of the marine environment; and little investigation on modeling the ocean features given its spatio-temporally dynamics. Based on these issues, localization and navigation of AUVs in such environments become not only challenging, but also nonlinear and uncertain. Motivated by these issues, we explored the concept of Ecological Niche Modeling, which is derived from one of the primary goals of ecology: to map species distribution over geographic ranges and be able to use predictive models to infer where various species are likely to be found [MM15, Sob10, KP09, MTMT09]. Environmental niche modeling uses a wide range of data to generate a map of a locale showing only chemical and physical parameters that have either been measured or interpolated from direct measurements $\left[\mathrm{REH}^{+} 11\right]$. Specifically, niche modeling is a method to classify geographic locales as either being habitable or inhabitable by certain species. By monitoring specific physical parameters of an environment and understanding the tol- 
erances of a certain species, it is possible to model where that species will most likely be present [MTMT09, Sto06, EL09, Pet06]. In other words, Environmental niche modeling is related to the possibility that the presence of certain species depends on physical environmental parameters of that location.It uses a wide range of data to generate the reference map of a locale and it shows only chemical and physical parameters that have either been measured or interpolated from direct measurements $\left[\mathrm{REH}^{+} 11\right]$. Following this idea, the representation of actual species distributions is unnecessary. What matters here is the prediction where a species may reside within that specific environment. In order to do so, the extents of physical parameters that the species can tolerate, i.e., , the temperature range within which a species can physically survive need to be understood. This methodology has the ability to probabilistically over-predict areas that the species may reside within compared to the area the species is actually occupying. In conclusion, it is important to stress that environmental niche models and species distribution models are different approaches used in order to the investigate and predict an accurately classifier of the distributions of species on a large scale.

In this research, we hypothesize that these niches may also be utilized for underwater vehicle navigation. Although the niches may move in space and time, there appears to be relative navigation information, which have the form of landmarks or hotspots, that can be exploited for prescribed regions of interest. In other words, we are interested in applying this concept for navigation and localization in regions where repeated sampling or revisits occur. This is also important for a further analysis over time and periodic variability in order to create a dynamic model of the environment through the application of deep learning and neural networks.

For its utility in navigation and localization of autonomous underwater vehicles, data would be ideally collected by these AUVs equipped with adequate sensors to characterize the physical parameters of the aquatic environment. Nevertheless, the importance here 
lies in the fact that environmental niche models are only as accurate as the data upon which it is based. Here, it is common practice to use specialized AUVs to collect the water data. The vehicles contain sensors and global positioning devices to correlate those parameters to precise geo-spatial locations. Also, it important to remember at this point that even the global positioning system can fail underwater and it is highly reliable on the water surface. Below the surface, AUVs need to rely on other localization methods. This problem of localization is a large hurdle to collecting data in an aquatic environment (as investigated deeper in Chapter 5).

At this stage, we will assume that the environment is static in both space and time, however the spatio-temporal dynamics of observed ecological niches suggests that they exhibit periodicity or a predictable stochastic behavior, see e.g., [SHLC16].

\subsubsection{Reference Maps in Localization}

In Robotics, robots usually use reference maps to navigate in large-scale environments and they have to handle several challenges e.g., path planning algorithms that are high computation intensive, insufficient knowledge about the environment, among others. In $\left[\mathrm{BFD}^{+} 17\right]$, a novel framework, named Topomap, is developed using a sparse featurebased map from a visual Simultaneous Localization And Mapping (SLAM) systems and transforming it into a three-dimensional topological map. This approach is shown to improve efficiency in global planning and obtained similar results as RRT* approaches with less computation time and memory. In $\left[\mathrm{HHL}^{+} 14\right]$, the authors presented a system on-board a helicopter developed using 3D occupancy grids acquired from stereo images in order to obtain a $3 \mathrm{D}$ representation of the environment. Also, in [STR $\left.{ }^{+} 13\right]$, the authors developed local 3D occupancy grid using the on-board visual odometry, reaching the conclusion that micro aerial vehicles (MAVs) can autonomously navigate in both in- 
door and outdoor environments solely relying on vision-based systems. Applications on vision-based 3D maps include inspection tasks such as mapping earthquake damaged buildings $\left[\mathrm{MSM}^{+} 12\right]$, where the authors also demonstrated that their system is operated entirely in teleoperation. Moreover, in [BOAS15], the authors presented a novel system for localization of micro aerial vehicles in unknown environments with just vision and IMU measurements and that runs in real-time, obtaining success in relocalization and estimation using the global map. In the underwater scenery, a navigation system for autonomous underwater vehicles (AUVs) navigating in dams, harbors, marinas, and marine platforms was developed using scanned imaging sonar in order to acquire information on the location of vertical planar structures and incorporated into a featurebased simultaneous localization and mapping (SLAM) algorithm in combination with an extended Kalman filter [RRTN08]. SLAM algorithms are motivated by the lack of high-quality and up-to-date maps in most of the underwater environments. SLAM can be understood as a probabilistic estimation problem applicable for both $2 \mathrm{D}$ and $3 \mathrm{D}$ motion and uses mapping in underwater environments can be found in [LB16].

\subsection{Augmented Terrain-Based Navigation Framework}

\subsubsection{Overview}

The basic process for creating a terrain map from the scientific and bathymetric data is to first generate a base map for each data parameter being collected. Then, determine a weighting schema that enables the maps to be brought together via linear combination while maximizing the contrast of the resulting terrain map. More specifically, the raw data are first treated for outliers with the k-nearest neighbor technique, and individual scalar fields are created for each data parameter. For filling in the spaces between points, 
a geostatistical technique, known as kriging, was used (See chapter 3). This technique has been successfully used to create regional distribution maps for the interpolation of nonpoint sources of physical parameters, e.g., a high-precision underwater digital elevation model was developed in [ZXX15], having concluded that kriging for terrain-aided navigation is better than other traditional interpolation methods in terms of accuracy. Other applications of this methodology include heavy-metal contamination in soil [HOBR14], air pollution maps for eastern China [RM15], concentration of chlorinated aliphatic hydrocarbons (CAHs) that contaminate shallow groundwater $\left[\mathrm{LLL}^{+} 15\right]$, and concentration of dissolved oxygen (DO) in wastewater treatment ponds [KIJT17].

When kriging is compared to the bi-harmonic spline interpolation ${ }^{1}$, as used in [RFB $\left.{ }^{+} 17 \mathrm{a}\right]$, kriging turn out to be less computational intense and five times faster in execution time for the same dataset. Having the maps for each water parameter with low errors in prediction, the next step it to find the appropriate weights for each one of these parameters and combine them in such a way that there is an increase in the uniqueness of individual positions in that environment. This increase of uniqueness presents an opportunity for navigation and localization with reference to unique features within a waterbody. Unique features can be used as fiducial markers or landmarks for relative navigation. This approach would enable an AUV to spend a larger amount of time dedicated to data collection underwater with increased accuracy without having increasing extra energy expenditure on surfacing for GPS fix.

\subsubsection{Global Correlation Score}

It is a novel technique that quantifies the contrast of a scalar field. It is determined by computing the $2 \mathrm{D}$ spatial auto-correlogram. The entry at $(0,0)$ is removed and the absolute

\footnotetext{
${ }^{1}$ MATLAB griddata method 'v4'
} 
values of the $2 \mathrm{D}$ auto-correlogram are summed together to give the auto-correlation. The hypothesis is that creating a scalar field with the lowest global correlation will produce a terrain map in which any trajectory through the underlying map is unique.

\subsubsection{Weighting Scheme}

The resulting maps of chapter 3 can be seen as a matrix, $\mathbf{X}$, where each element $(\mathrm{i}, \mathrm{j})$ corresponds to the coordinate $(\mathrm{x}, \mathrm{y})$ where the value was measured. Using interpolated data, we create a scalar field with with small global correlation.

\section{Problem Formulation}

We consider a marine environment as a workspace that is divided into a set of layers $L$ based on different depths of the environment. At each layer, the environment is discretized into a $2 \mathrm{D}$ grid map. Let $\mathscr{O}$ be the land region of the environment at a specific layer where the drifter cannot navigate. The free water space of the marine environment at each layer is denoted as $E$ excluding the land region.

\section{Problem 1. Weighting scheme with no prior knowledge on weight distribution:}

Given an aquatic environment $E$ discretized by a map $M$, where each cell $m_{t}^{j}$ is given by a geographic coordinate (lat,long) and a sensor derived value $z_{t}^{j}$ at a time $t$, find the appropriate weights $y_{k}$ for each water parameter $k$ sensed by the robot such that the global correlation $G C(M)<G C\left(M_{d}\right)$, where $M_{d}$ is a map consisting of only bathymetric information and there is no knowledge on the initial distribution of weights for $k$ parameters.

Based on Kraemer Algorithm [ST04], suppose we wish to select $X=\left\{x_{1}, \ldots, x_{n-1}\right\}$ with unique entries from a uniformly random sampling over a multinomial distribution $\{1,2, \ldots, D-1\}$ without replacement, such that: 


$$
0=x_{0} \leq x_{1} \leq x_{2} \leq \ldots \leq x_{n-1} \leq x_{n}=D
$$

where $D$ is a sufficiently large integer. In order to address the problem of sampling uniformly from a unit simplex from a simplex, we use the following algorithm:

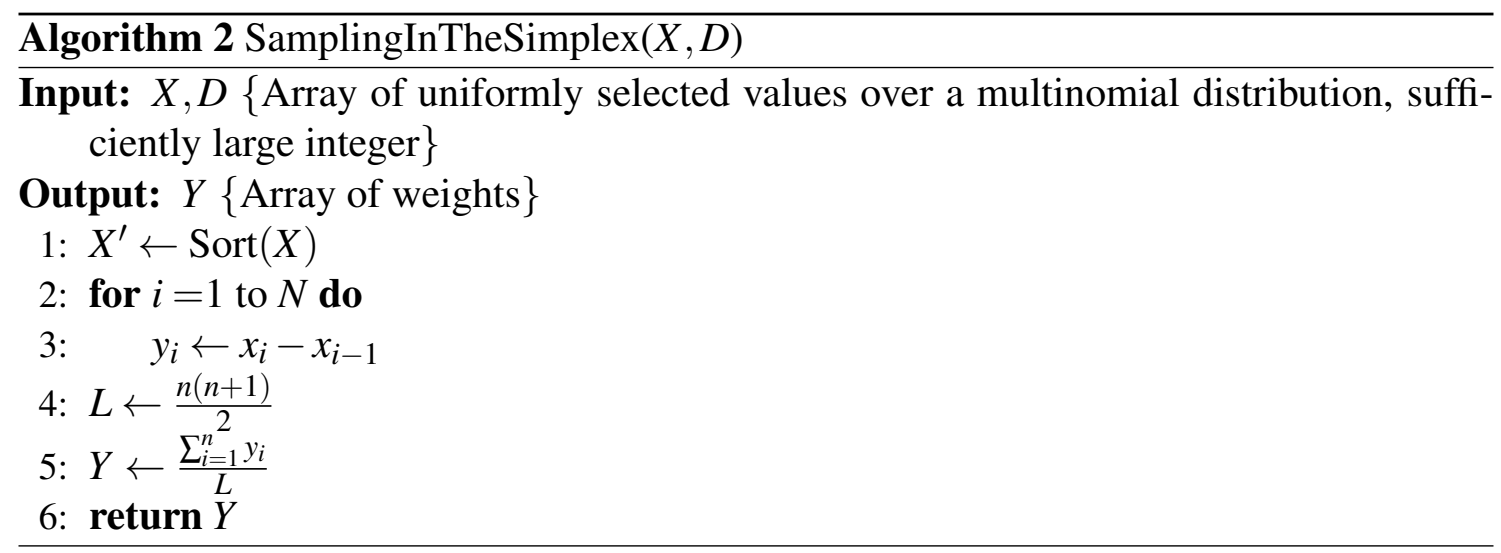

In algorithm 2, line 3, it is important to highlight that $\sum_{i=1}^{n} y_{i}=D$. This algorithm has the property of producing a point in the unit simplex.

$$
\begin{aligned}
S= & \alpha_{1} * \text { salinity }+\alpha_{2} * \text { temperature }+\alpha_{3} * \text { specific_conductivity }+\alpha_{4} * p H+ \\
& \alpha_{5} * \text { turbidity }+\alpha_{6} * \text { chlorophyll }+\alpha_{7} * \text { blue_green_algae }+\alpha_{8} * \text { dissolved_oxygen }
\end{aligned}
$$

Here, $\mathrm{S}$ represents a linear combination of the science parameters considered, $\alpha$ is the set of coefficients that minimizes the spatial auto-correlation.

For the third test, only bathymetric information is considered. An augmented TBN has been developed using depth data in [SLS15] and results showed that bathymetric information is a viable approach for creating terrain maps. Here, we examine bathymetric information using the same methods used for addressing questions 1,2 and 4. Finally, the fourth and last test is addressed by combining the bathymetric infomation with the science parameters as a new approach. Equation 4.2 is extended to include depth as another variable and analyzing the effect of the bathymetric structure. 
The robot's position $\hat{x}_{t}$ at time $t$ and is given by time $t-1$ and its control input $u$ :

$$
\hat{x}_{t}=f\left(x_{t-1}, u_{t}\right)
$$

Based on the procedures for mobile robot localization using Kalman Filter Localization in [SN04], we can describe the sensor measurements by array $z_{t}^{i}$ at a time $t$, where $i=0 . . n$ for $n$ different observations. By using the robot position $\hat{x}_{t}$ and the map $M$ to generate several predicted feature observations $z_{t}^{j}$.

\section{Problem 2. Weighting scheme with prior knowledge on initial weight distribu-} tion:

Given an aquatic environment $E$ discretized by a map $M$, where each cell $m_{t}^{j}$ is given by a geographic coordinate (lat,long) and a sensor derived value $z_{t}^{j}$ at a time t, find the appropriate weights $y_{k}$ for each water parameter $k$ sensed by the robot such that the global correlation $G C(M)<G C\left(M_{d}\right)$, where $M_{d}$ is a map consisting of only bathymetric information and $y_{0}^{0}, y_{0}^{1}, \ldots y_{k}$ are the initial weights for $k$ parameters.

Here, a weighting schema for the parameters is determined through Dirichlet Distribution, which is a reference distribution to model vectors of weights adding to 1 . It is a probability density function over the simplex and can model prior knowledge of the weights of the parameters. Based on the detailed tutorial in [Pai], let $\pi$ be a finite $D$ dimensional vector such that $0 \leq \pi_{i} \leq 1$, for $i=0, \ldots, D$ and $\sum_{i=1}^{D} \pi_{i}=1$. This vector is the parameter for the multinomial distribution where $X \sim \operatorname{Mult}(\pi)$ for $X \in 1, \ldots, D$ with probability $P(X=i \mid \pi)=\pi_{i} . \pi$ can be found using the Dirichlet Distribution density function given by:

$$
\mathbf{p}\left(\pi \mid \beta_{1}, \ldots, \beta_{D}\right)=\frac{\Gamma\left(\sum_{i} \beta i\right)}{\prod_{i} \Gamma(\beta i)} \prod_{i=1}^{D} \pi i^{\beta i-1}
$$


where $\pi$ is the vector of parameters of the Dirichlet distribution with $\beta_{i}>0 \forall i$. However, we model $b_{e t} a_{i}$ as a prior guess given by its equivalent $\alpha \equiv \sum_{i} \beta_{i}$ and $g_{0 i} \equiv \frac{\beta_{i}}{\sum_{i} \beta_{i}}$, where $g_{0}$ and $\alpha$ control the distribution behavior (tightness):

$$
\mathbf{p}\left(\pi \mid \alpha g_{0} 1, \ldots, \alpha g_{0} D\right)=\frac{\Gamma(\alpha)}{\prod_{i} \Gamma\left(\alpha g_{0} i\right)} \prod_{i=1}^{D} \pi i^{\left.\alpha g_{0} i\right)-1}
$$

As a result of this approach shown in [Pai], when $\alpha=D$, the density if uniform in the simplex; when $\alpha>D$, the density is clustered around $g 0$ and when $\alpha<D$, the density is sparse.

The weighting scheme for the parameters to give the lowest autocorrelation is determined through Dirichlet Distribution, which is a reference distribution to model vectors of weights adding to 1 . It is a probability density function over the simplex and can model prior knowledge of the weights of the parameters. The Dirichlet Distribution is defined as:

$$
\mathbf{p}(w \mid \alpha)=\frac{\Gamma\left(\sum_{i=1}^{n} \alpha_{i}\right)}{\prod_{i=1}^{n} \Gamma\left(\alpha_{i}\right)} \prod_{i=1}^{n} w_{i}^{\alpha_{i}-1}
$$

where $\alpha$ is the vector of parameters of the Dirichlet distribution with $\alpha_{i}>0 ; w=\left\{w_{1}, w_{2}, \ldots, w_{n}\right\}$, $w_{i}>0$ and $\sum_{i=1}^{n} w_{i}=1$, is the vector in the $\mathrm{n}$-dimensional probabilistic simplex representing the weights of the parameters; $n$ is the number of parameters to be considered for the generation of the scalar fields and $\Gamma$ denotes the gamma function. The individual scalar fields from each parameter are brought together via a linear combination with their respective weights to create a single scalar field that is the terrain map. This map is given by $S=w_{1} * v a r_{1}+w_{2} * v a r_{2}+\ldots+w_{n} * v a r_{n}$. The weighting schema is iterated on until the global correlation converges to a minimum value.

Any traditional TBN algorithm can then be applied to this augmented terrain map. It is also assumed that the combination of multiple parameters will produce a terrain map that is more unique than a terrain map composed of a single parameter; thus, improving the ability to reduce navigation uncertainty while underwater. The complete technique of 
ATBN would involve surveying an area, determining the weights of data parameters, and generating a terrain map through post processing. The vehicle is then provided with the terrain map and weightings to perform ATBN during subsequent deployments. Research into how to efficiently update this underlying map is presented in [MCSed].

The utility of our proposed method is currently assessed by localizing a trajectory within a computed augmented terrain map. Here, we conduct a total coverage path for a region of interest to compute the underlying ATBN map. To eliminate bias, we use a trajectory from a mission run at a later time for assessing navigational accuracy.

\subsubsection{Spatial Autocorrelation}

In Statistics, spatial autocorrelation is an important concept that evaluates the similarity between nearby observations. In datasets, specially geographic ones, measured datapoints at nearby locations may have closer values than measured datapoints at locations that are farther apart i.e., measured datapoints at different locations may not be independent.

According to [Leg93], autocorrelation is a general characteristic of ecological variables, specially the ones observed along time series (temporal autocorrelation) or across geographic space (spatial autocorrelation).

There are usually two groups of methods to assess the spatial structures of variables: a point pattern analysis and a surface pattern analysis [Gri13]. The first deals with datasets where the distribution of individual objects across a geographic space is more important than the overall spatially continuous distribution, which is the case for the second group. Moreover, in the point pattern analysis, one of the goals is to infer and interpret the kind of process that generated such structure. For more references on the analysis and application of point patten analysis, see [Pie77], [MFC77], [GB78], [WAM04], [Rip81], [WM13], 
[Rip87], [GBDR96] and [UF $\left.{ }^{+} 85\right]$. For further references on the surface-pattern analysis, see [CO81], [Rip81], [UF $\left.{ }^{+} 85\right]$, [UF89] and [LF89].

The concept of spatial autocorrelation has been used in different applications. In $\left[\mathrm{ECR}^{+} 06\right]$, the authors use spatial autocorrelation and variance of remote sensing data to develop region-growing segmentation algorithms with the objective of selecting the appropriate parameters for the algorithms. Moreover, the literature currently does not provide many resources about which how to choose appropriate ways (models) of describing the spatial arrangement of the data and which models work best in each situation. Therefore, in [Dub98], the author does a comprehensive study on spatially autocorrelated error terms in modeling the spatial distribution of observations in a dataset.

The goal to use spatial autocorrelation in the context of this research is to give a score for the spatial randomness of the fields we wish to construct. These fields are derived from bathymetric data and water parameters e.g., temperature, salinity, dissolved oxygen, turbidity, among others.

In more details, spatial autocorrelation measures the correlation of a variable with itself through space, describing the degree in which two observations at spatial locations are similar to each other. In order to measure spatial autocorrelation we need two things: observations and locations. Usually, spatial autocorrelation can have a positive or negative value. Positive spatial autocorrelation interprets similarity values in neighboring locations as occurring more often than in spatial randomness. One might think of this case as an impression of clustering, or clumps of like values.

Negative spatial autocorrelation occurs when dissimilar values occur near one another. It resembles the pattern of a checkerboard. It is important to highlight that there will be clustered patterns even in a spatial random system, but they will not occur very often. The big question here is how to quantify a positive, negative and random spatial autocorrelation. 
While spatial autocorrelation is a measurement that identifies similarity or dissimilarity in space, the spatial randomness interprets this location as having measured values not dependent on other measured values at neighboring locations. In this case, if certain values at different locations are altered, the overall content will not be affected given the fact that observed spatial pattern of the measured values is equally likely as any other spatial pattern.

In order to quantify the spatial autocorrelation of a certain field, it is important to first interpret its similarity (or dissimilarity) based on its observations at nearby locations. This measurement is dependent on the attribute similarity (which capture the similarity within one variable, but in nearby locations) and the locational similarity. A common technique to measure attribute similarity is the cross product of values observed at two locations:

$$
y_{i} \cdot y_{j}
$$

for all pairs of points $(i, j)$ in the dataset in nearby locations. The cross product is said not to be large or small. On the other hand, to measure dissimilarity is given by the absolute difference:

$$
\left|y_{i}-y_{j}\right|
$$

for all pairs of points $(i, j)$ in the dataset in nearby locations. In this case, the smaller the difference, the more similar they are. Under randomness there will not be systematically large or small values. To visualize this in the context of this research, we can think about the following problem:

\section{Problem 3. Dissimilarity measurement of scalar fields:}

Given an aquatic environment $E$ discretized by a map $M$, where each cell $m_{t}^{j}$ is given by a geographic coordinate (lat,long) and a sensor derived value $z_{t}^{j}$ at a time $t$, interpret the spatial autocorrelation using the absolute difference given by 4.3.4. 


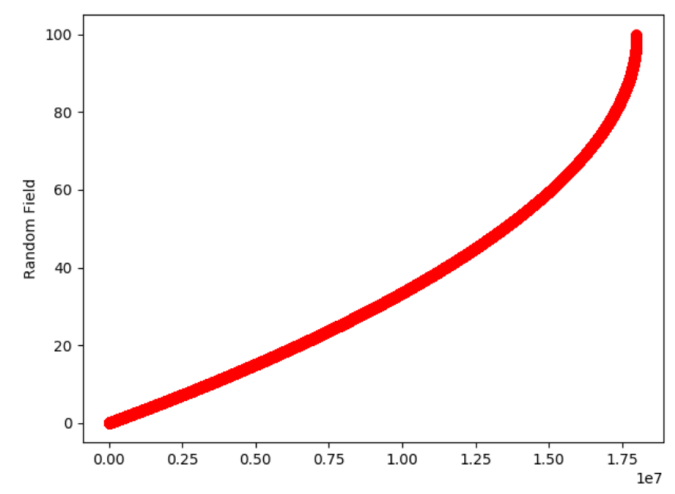

Figure 4.1: Dissimilarity measure based on for a random field.

If we think about a random field and compute for all neighbor observations, it is expected that resulting values are different for each pair $(i, j)$. If we plot the computed differences between each pair $(i, j)$ in ascending order, it is possible to have a visual dissimilarity interpretation of the field. Since, for the case of random fields, the differences are expected to be different, the plot of these differences in ascending order should have a shape as shown in figure 4.1 .

Dissimilarity measure for the collected datasets are shown in figure 4.2. By visually interpreting the dissimilarity of these fields, the dissolved oxygen, turbidity, depth and temperature parameters seem to have higher number of dissimilar observations, suggesting a spatial autocorrelation closer to 0 and i.e., resembling more the shape of a random field 4.1 than salinity and $\mathrm{pH}$, which have a higher number of similar differences, suggesting a positive or negative spatial autocorrelation.

The locational similarity is dependent on the notion of neighboring or spatial weights $\left(w_{i}\right)$. Spatial weights matrices are an essential component in most regression models where a representation of spatial structure is needed [GA10]. This concept has been widely used for finding spatial patterns of the distribution of householdsín clusters of cities [PT02], identification of spatial anomalies and their spatial patterns in disease regions [TLCP09], population change [CZ08], but also used in social networks [LLRX06]. 


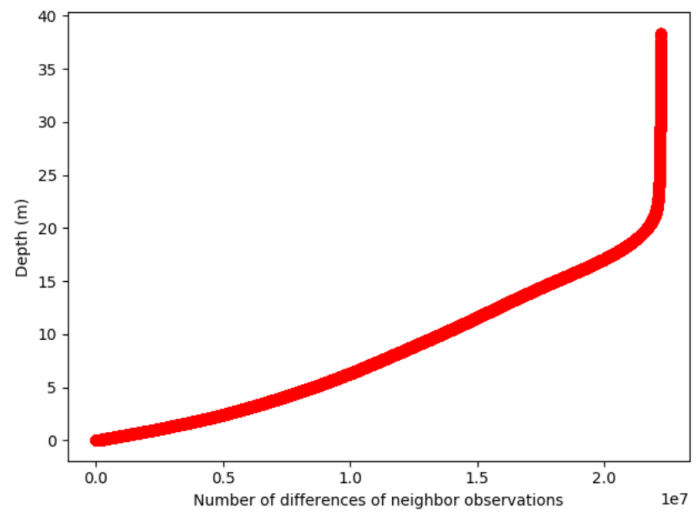

(a)

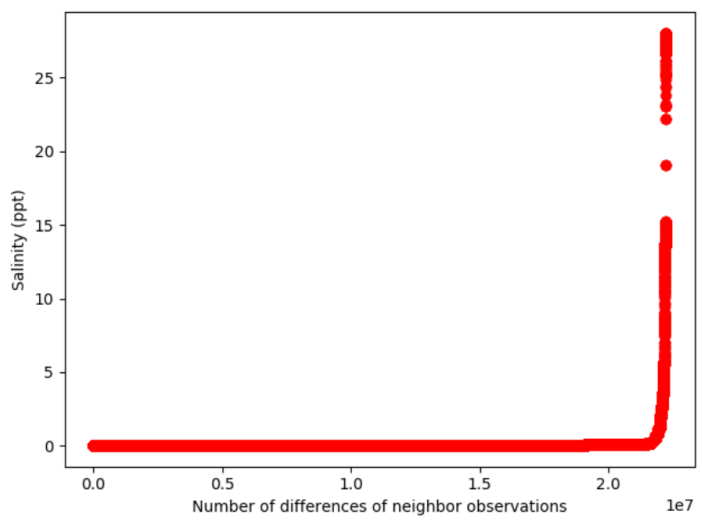

(c)

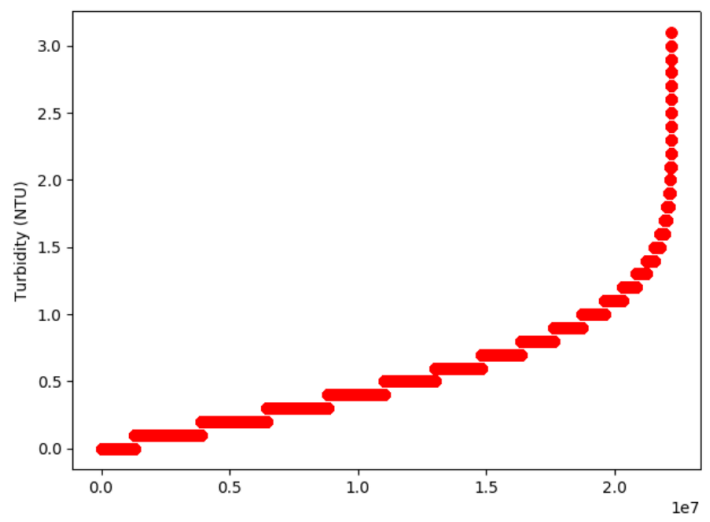

(e)

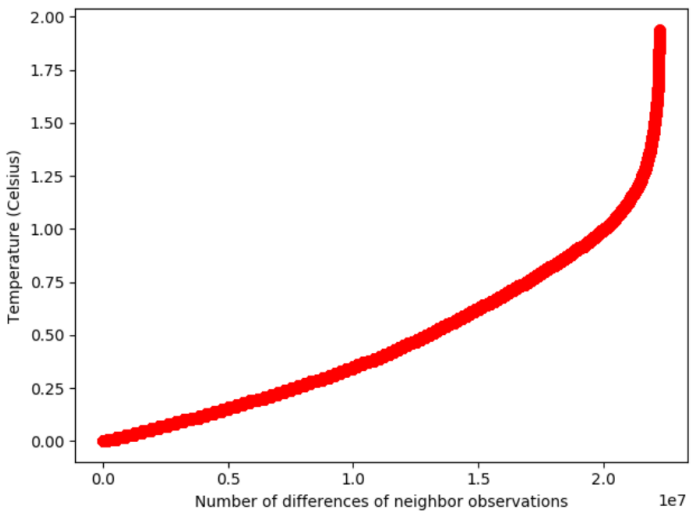

(b)

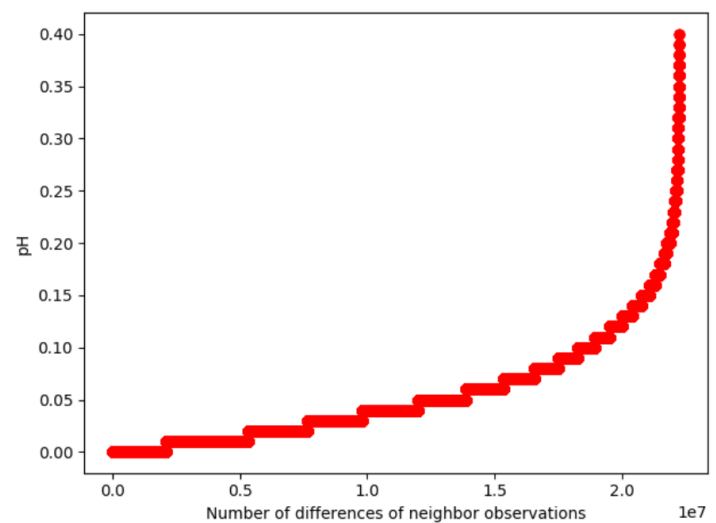

(d)

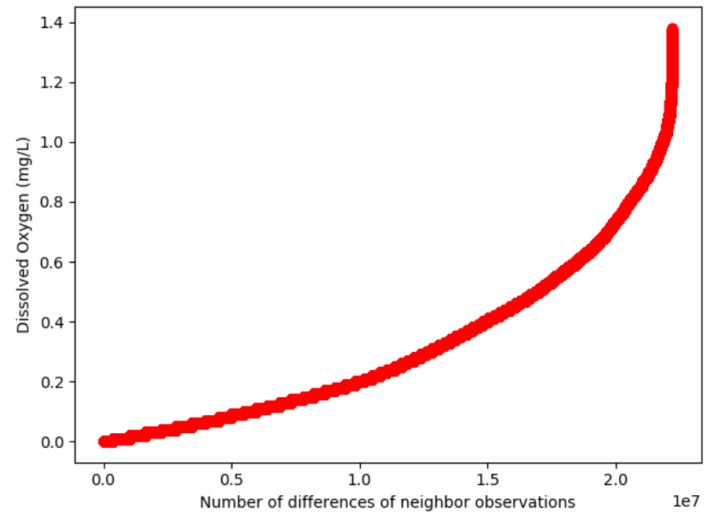

(f)

Figure 4.2: Semivariograms of the water parameters depth, temperature, salinity, $\mathrm{pH}$, turbidity and dissolved oxygen generated in the datasets collected in Santa Catalina Island on July 13th 2016 (left images) and July 14th 2016 (right images). 
In [GA10], the authors construct a spatial weights matrix that is based on local statistics model (LSM) and spatial structure from which some units evoke a distance effect, and some do not, having concluded that local statistics model perform better than global models due to its flexibility to deal with spatial weights matrices.

Spatial autocorrelation deals with dependence of spatial interactions; therefore, the spatial weights should be identified by a $N \times N$ matrix $W$ with elements $w_{i j}$ such that $w_{i j}>0$ for neighbors and $w_{i j}=0$ when $i$ and $j$ are not neighbors and $w_{i i}=0$ meaning that there is no self-similarity. A classic approach for these weights are based on binary contiguity, where $w_{i j}=1$ if $i$ and $j$ are neighbors, and $w_{i j}=0$ otherwise. In other words, for each observation, all other observations are potential neighbors and a neighbor can be defined using such as rook or queen contiguity criteria. To illustrate these criteria, consider the following matrix:

The neighbors of $E$ are $\{B, D, F, H\}$ following the rook criterion and $\{A, B, C, D, F, G, H, I\}$ following the queen criterion.

Another approach for find the appropriate weights is using the distance between the datapoints (or polygon centroids in a certain region), which is one of the methods for 2dimensional interpolation described in Chapter 3.2. In this approach, $w_{i j}>0$ for $d_{i j}<d$ for a certain critical distance $d$ from each other. Moreover, it is also possible to identify the $k$-nearest neighbors, which is irrespective of distance, but assigns the same number of neighbors for all observations (leading to a tie problem). 


\subsection{Preliminary Results}

\subsubsection{Sampling in the Simplex and Global Autocorrelation Score}

In this section we will present the preliminary results for the weighting scheme using the method of sampling in the simplex and using the Global Correlation score.

Results for all possible combinations of two science variables show that when salinity and turbidity are combined as follows:

$$
2 \text { SciVar }=0.38 * \text { salinity }+0.62 * \text { turbidity }
$$

the global correlation value is 14838.49 . This value is minimum compared to all other combinations of two science parameters. The terrain map for this approach and its autocorrelogram in Figure 4.3. This result indicates the viability of using science parameters for terrain-based navigation since there is a high variability for the terrain map generated.

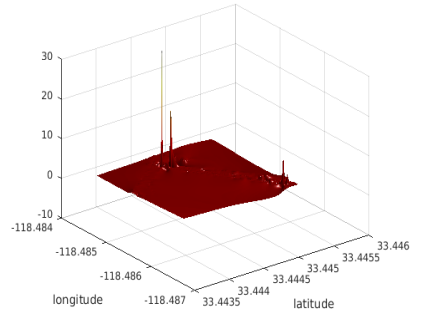

(a)

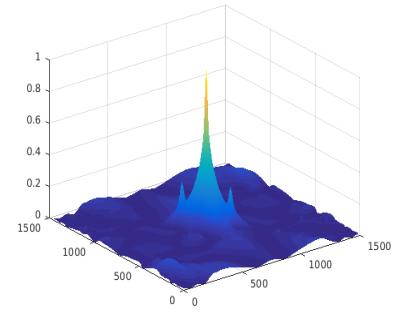

(b)

Figure 4.3: For the combination of two science parameters with maximum variability: (a) terrain map; (b) auto-correlogram.

By uniformly picking one million points from a simplex and comparing the global correlation of each combination, results show that the minimum global correlation is 8062.21 after testing for one hundred thousands different set of coefficients. The best global correlation was achieved when:

$$
\alpha_{1}=0.226828 \text { (Salinity) }
$$




$$
\begin{aligned}
& \alpha_{2}=0.190777(\text { Temperature) } \\
& \alpha_{3}=0.096279(\mathrm{pH}) ; \\
& \alpha_{4}=0.450075 \text { (Turbidity); } \\
& \alpha_{5}=0.00025 \text { (Chlorophyll); } \\
& \alpha_{6}=0.023924 \text { (Blue-green algae); } \\
& \alpha_{7}=0.011867 \text { (Dissolved oxygen). }
\end{aligned}
$$

According to this approach, the turbidity of the water, measured in Nephelometric Turbidity Units (NTU), has the highest coefficient and it is the variable that leads to higher variability of the terrain map, desired for the TBN approach. The terrain map for this approach and its auto-correlogram are illustrated in Figure 4.4.

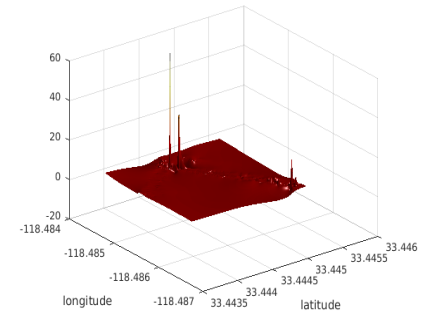

(a)

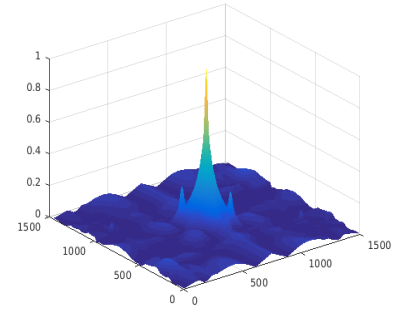

(b)

Figure 4.4: For the combination of the seven science parameters with minimum autocorrelation: (a) terrain map; (b) auto-correlogram.

In the case of using only the bathymetry information, global correlation is approximately 610096.77. This approach was examined in [SLS15], and results demonstrated an accurate localization of a trajectory traversed by an underwater vehicle when water depth information correlated to local bathymetry maps was used. The auto-auto-correlogram for this case is illustrated in Figure ??. The last and most important results shows that when using a combination of science parameters and the bathymetry data, global correlation value is 3879.67 . 


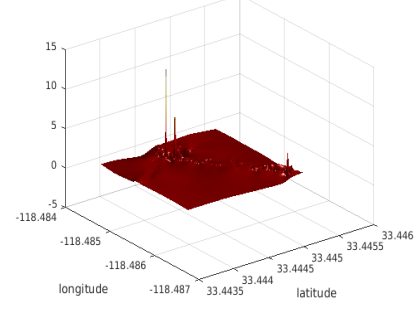

(a)

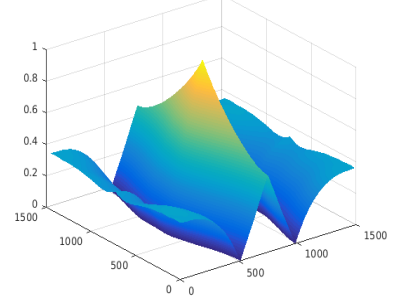

(b)

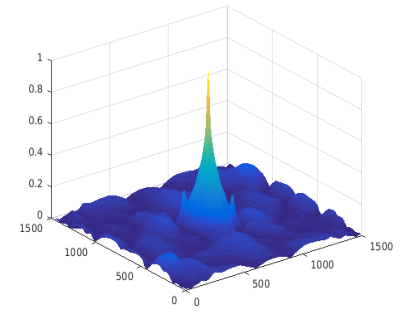

(c)

Figure 4.5: Terrain map generated for the combination of science parameters and bathymetric information (a), auto-correlation for the bathymetric information (b) and autocorrelation for the combination of science parameters and bathymetric information (c).

Results show that the minimum auto-correlation is achieved when the science parameters are combined with bathymetric information. When only science parameters are used, auto-correlation is also lower than just bathymetric information, justifying the use of science parameters for creating terrain maps for localization and navigation. The combination of science parameters and bathymetry data led to significant reduction in the global correlation when compared to only using bathymetry information. This result, in turn, increases the variability and facilitates localization and navigation since any random trajectory extracted from the terrain map will be unique in this body of water. It is known from [SKS16] and [SLS15] that TBN and the use of bathymetry information work well for localization and navigation because the structure of the bathymetry facilitates the unique segment of a trajectory to be found. When combining more science with bathymetry information, the generated scalar fields terrain maps are optimized for a TBN.

Table 4.1 shows the global correlation values for the best variability achieved for a combination of two science parameters; best combinations of the seven science parameters; only the bathymetry information and the combination of the seven science parameters and the bathymetry information. 
Table 4.1: Global correlation values for different combination of parameters.

\begin{tabular}{|l|l|}
\hline Parameters & $\begin{array}{l}\text { Global correla- } \\
\text { tion }\end{array}$ \\
\hline $\begin{array}{l}\text { Salinity and Tur- } \\
\text { bidity }\end{array}$ & 14838.49 \\
\hline $\begin{array}{l}\text { Science com- } \\
\text { bined }\end{array}$ & 8062.21 \\
\hline Bathymetry and & 610096.77 \\
\hline $\begin{array}{l}\text { Science } \\
\text { bathymetry }\end{array}$ & 3879.67 \\
\hline
\end{tabular}

\subsubsection{Dirichlet Distribution and Global Autocorrelation Score}

Using the data from on-board sensors, terrain maps for localization and navigation were generated for use in later missions. Preliminary results using bathymetric data in [SLS15] showed that global correlation is low. When physical water parameters are combined with bathymetric information instead of using either one independently, a lower global correlation value is obtained. Results in different bodies of water are shown as follows.

\section{The Big Fisherman's Cove, Santa Catalina Island, California}

Depth and temperature are the most significant parameters in the ocean, in the case of the Big Fisherman's Cove, Santa Catalina Island, California, USA, when the aim is higher variability. Salinity represented $0.43 \%$ of importance in generating the most adequate scalar field, while temperature represented $18.7 \%$, turbidity $0.26 \%$, depth $80.6 \%$ and $0.01 \%$ for the remaining variables. Fig. 4.6-b shows the auto-correlogram of the scalar field and Fig. 4.6-a show the actual scalar field for the surveyed area at the Big Fisherman's Cove. 


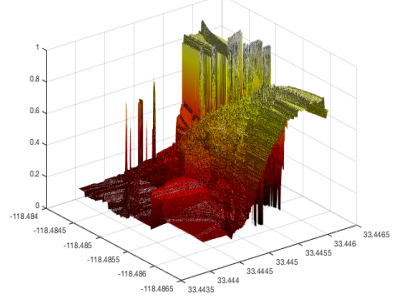

(a)

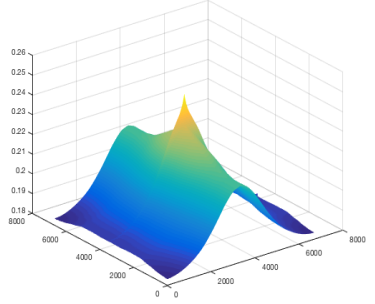

(b)

Figure 4.6: Scalar field map at the Big Fisherman's Cove, Santa Catalina Island (a) and its auto-correlogram (b).

\section{Lake Nighthorse, Colorado}

Depth and temperature are the most significant parameters in the case of Lake Nighthorse, Durango, Colorado, USA (large fresh water lake) when the aim is higher variability. Depth represented $69 \%$ of importance in generating the most adequate scalar field, while temperature represented $30 \%$, and $1 \%$ for the remaining variables. Fig. 4.7-b shows the auto-correlogram of the scalar field and Fig. 4.7-a shows the actual scalar field for the surveyed area at Lake Nighthorse.

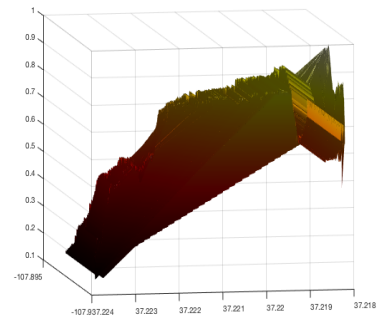

(a)

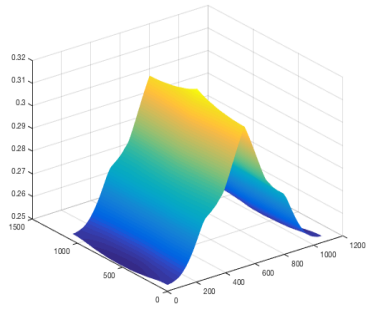

(b)

Figure 4.7: Scalar field map at the Lake Nighthorse, CO (a) and its auto-correlogram (b).

Results showed that for coastal ocean bay, as seen at the Big Fisherman's Cove, in California, USA, the parameters depth, temperature and salinity are among the most important ones. The importance is due to the effect on the global autocorrelation that be- 
comes lower when these parameters have a certain weight in the construction of the scalar field.

\subsubsection{Discussion on Spatial and Temporal Terrain Map Analysis}

The primary issue that arises in using the proposed ATBN methodology is that the physical water parameters, e.g. temperature and salinity, are spatiotemporally dynamic. Thus, a generated terrain map based solely or partially on these variables will change in space and time. Here, we present initial investigations into the variability of these ATBN maps over many deployment locations across multiple time scales. Specifically, we present data from deployments of a YSI EcoMapper AUV in multiple locations over multiple time scales. During these deployments, both physical water parameters and bathymetric data were gathered on the surface.

An assessment of the weighting scheme of the terrain map methodology is presented. Here, we use data from a deployment in Monterey Bay $\left(36^{\circ} 48^{\prime} \mathrm{N} 121^{\circ} 47^{\prime} \mathrm{W}\right), \mathrm{CA}$, USA (coastal ocean bay), where the vehicle also navigated on the surface of the water to ground-truth measurements via GPS collecting data referenced to GPS locations, as a comparative for the weights calculated for Santa Catalina Island and Lake Nighthorse. The following sections examine the variability in weighting assignment to individual parameters over both space and time. We assume a quasi-static (multiple hours) environment persists within the survey area to create an initial coverage survey. We then compare the terrain maps created from these initial surveys over multiple deployments to observe changes in the parameter weightings. As many field deployments occur in an area over the period of days, weeks, and even years, understanding how the environment is changing, and how to efficiently update an underlying map would extend deployment times and optimize data collection activities. 


\section{Temporal Analysis}

We begin by examining the change in parameter weights with respect to time. In Fig. 4.8, we present an ocean deployment (Santa Catalina Island) and a fresh water lake deployment (Lake Nighthorse).
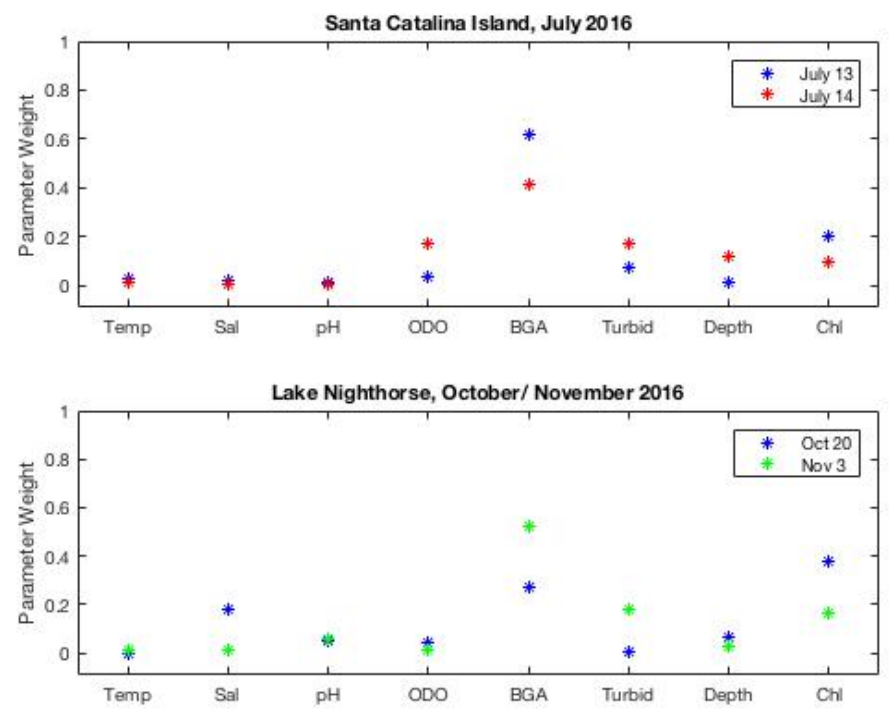

Figure 4.8: The individual parameter weights for two deployments on different days for both ocean (Top) and freshwater (Bottom) regions.

For the ocean region, the temporal difference in deployments is one day, and for the freshwater region the temporal difference is two weeks. As expected, the computed parameter weights for each of the four deployments are unique. However, we do see similarities within each region, as well as a noticeable trend with which parameters receive higher weights across all four deployments.

In the data from the ocean region, we see a nearly identical, and relatively small weighting $(\sim 0)$, computed for Temp, Sal, $\mathrm{pH}$, and Depth. A higher weighting $(\sim 0.6)$ is assigned to BGA. The other parameters received a moderate weighting $(\sim 0.1)$ with the variation in weights more significant than the maximum and minimum weights. This trend demonstrates repeatability in the computation method to optimize weights over mul- 
tiple parameters. Additionally, we note that over the course of approximately 24 hours, the same underlying terrain map could be used for accurate navigation, as the trend in weights across the parameter set is quite similar.

For the freshwater region, we see a nearly identical, and relatively small weighting $(\sim 0)$, computed for Temp, $\mathrm{pH}, \mathrm{ODO}$, and Depth. A higher weighting $(\sim 0.3-0.5)$ is computed for BGA and Chl. The Turbidity and Sal parameters received a moderate weighting $(\sim 0.15)$. Again, we note that over the course of 2 weeks, the same underlying terrain map could be used for navigation, as the trend in weights across the parameter set is quite similar, although there is much more variability in the fresh water dataset.

It is clear that the weighting of parameters is more similar with a shorter duration between deployments, however the trend in parameter weighting is obvious. Note the similar trend in parameter weights across both fresh and salt water deployments. This is evidenced in Fig. 4.9 with all four deployments plotted together.

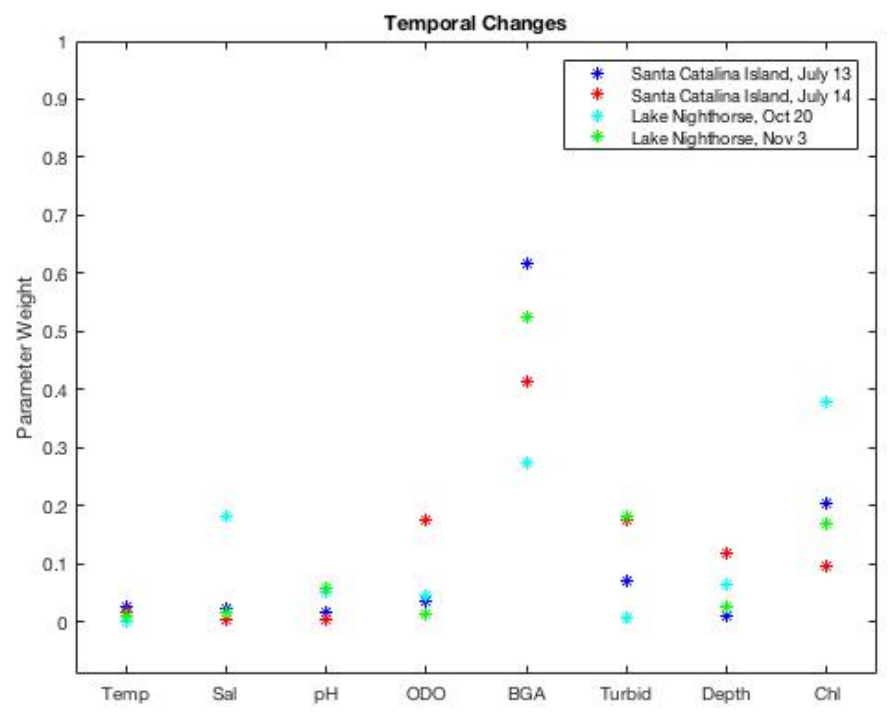

Figure 4.9: Individual parameter weights for four deployments covering both ocean and freshwater regions on different days. 


\section{Spatial Analysis}

Given the temporal trends that were evident in the previous section, it is of interest to examine whether those same trends are seen spatially as well. For this analysis, we examine two datasets from ocean regions; Santa Catalina Island and Monterey Bay. These two ocean regions are significantly different in that the bay off of Santa Catalina Island is relatively shallow ( $\sim 20 \mathrm{~m}$ at the deepest point) and sheltered. The deployment region in Monterey Bay is unsheltered and reaches depths of $>150 \mathrm{~m}$; the bay itself reaches depths near the deployment region of $>500 \mathrm{~m}$. Fig 4.10 displays the parameter weights for a single deployment in these two separate ocean regions.

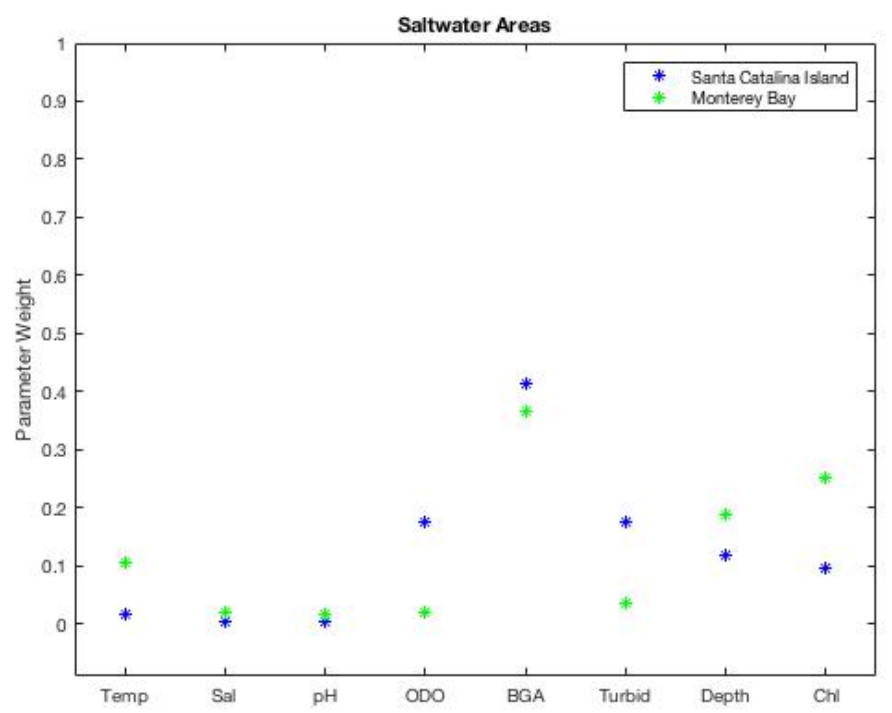

Figure 4.10: Individual parameter weights for two deployments covering different ocean regions at different times; Santa Catalina Island vs. Monterey Bay.

The datasets presented were gathered almost four years apart, with the Santa Catalina Island dataset gathered in July and the Monterey Bay dataset gathered in December. We remark that water temperatures were significantly different, and Chl, ODO and Turbidity were not correlated, across the two regions analyzed. In both cases the BGA has a relatively high weighting $(\sim 0.4)$, ODO, Turbidity, Depth and Chl have a moderate weighting 
$(\sim 0.2)$, while Sal and $\mathrm{pH}$ have relatively small $(\sim 0)$ weight. Regardless of these differences in absolute parameter values, we note a similar relative trend in the computed weights for the individual parameters as previously seen. This reinforces the hypothesis that there is a relative weighting between parameters that exists across deployment regions, as well as through time. It is also of interest to note that during the data collection in Monterey Bay, the EcoMapper constantly undulated between the surface and $14 \mathrm{~m}$ depth, while the data from Santa Catalina Island were all collected on the surface. This presents an interesting scenario for the analysis of 2-D versus 3-D terrain map creation for extending the proposed methodology. This analysis is outside the scope of this current work.

Te analyze the results of the weighing scheme using Dirichlet Distribution and Moran's I correlation coefficient for measuring the overall spatial autocorrelation of the individual and combined reference maps.

\subsection{Conclusion}

A map constructed using in situ science data in combination with bathymetric was developed for improved navigation and localization accuracy for aquatic vehicles. The incorporation of science data increased the global correlation leading to greater variability and a more suitable map for localization and navigation using an augmented TBN. The methods presented in this paper can serve as an important technique to create a terrain map with maximum variability across the range of data available.

However, this research examined only one deployment in a coastal ocean region and the parameters associated with this location will be unique to this region. Therefore, any random trajectory extracted from the terrain map will be unique to that area. This is what makes localization possible though an augmented TBN. When satellite navigation is un- 


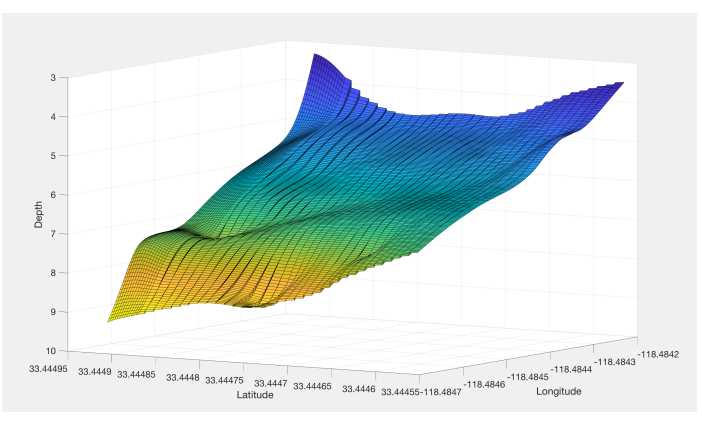

(a)

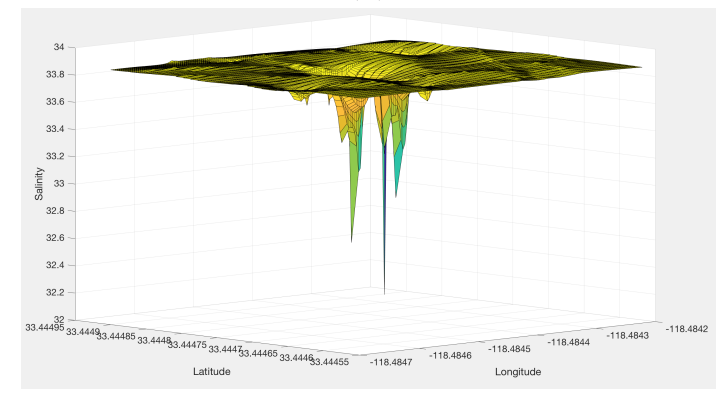

(c)

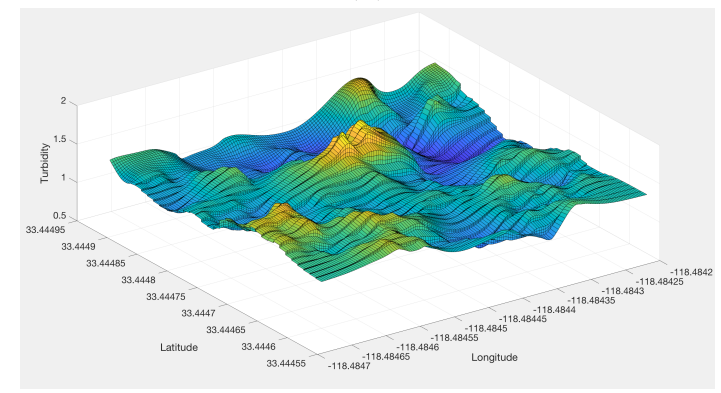

(e)

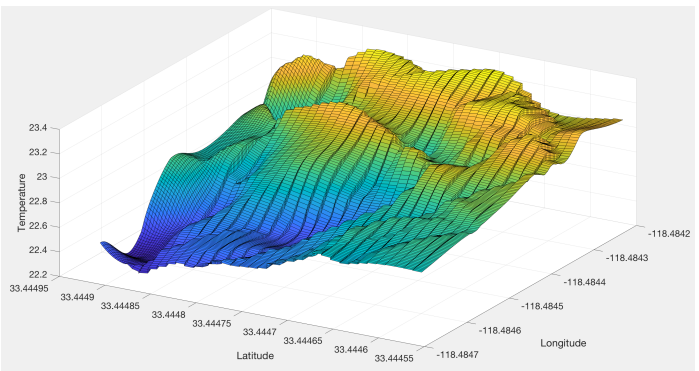

(b)

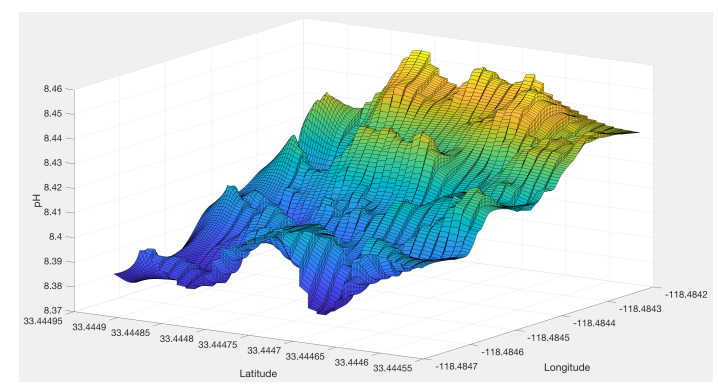

(d)

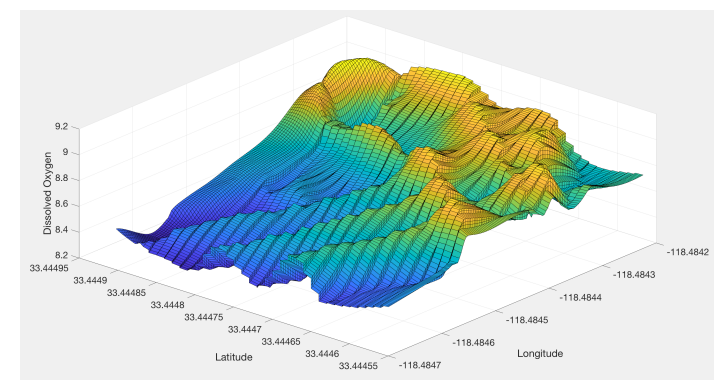

(f)

Figure 4.11: Final terrain maps computed for the following water parameters on July 13th 2016 in Santa Catalina Island, CA: depth (a), temperature (b), salinity (c), pH (d), turbidity (e) and dissolved oxygen (f) 


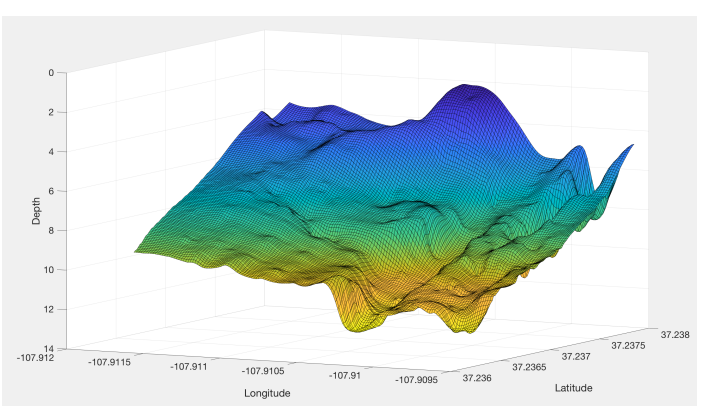

(a)

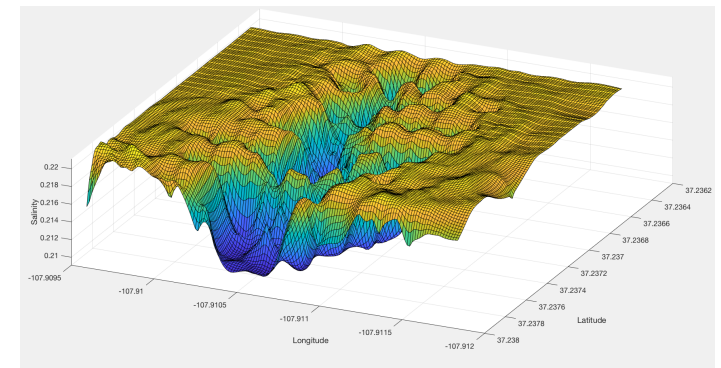

(c)

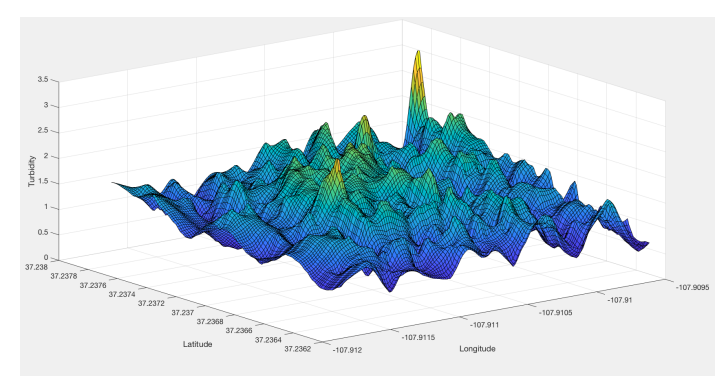

(e)

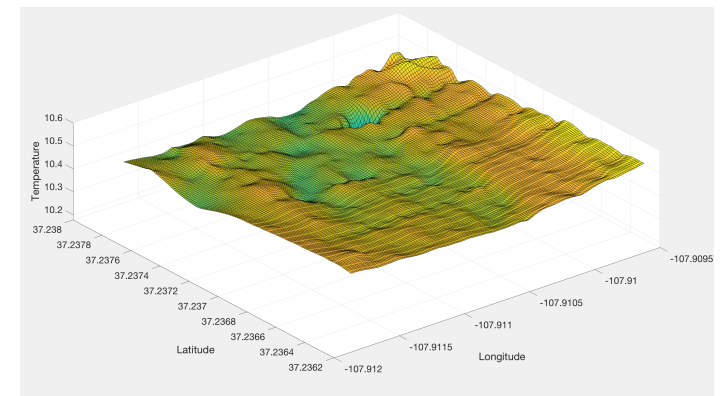

(b)

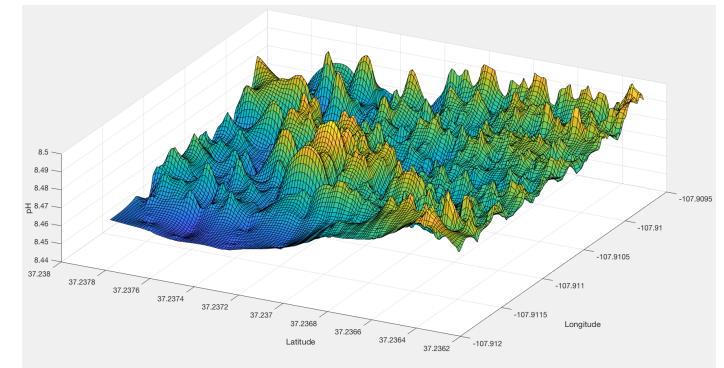

(d)

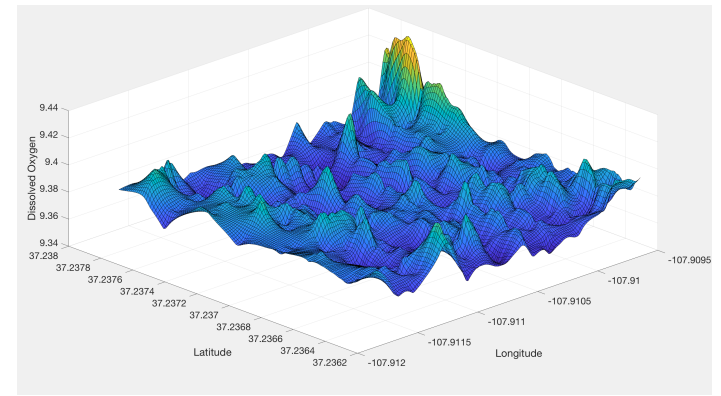

(f)

Figure 4.12: Final terrain maps computed for the following water parameters on May 2nd 2018 at Lake Nighthorse, CO: depth (a), temperature (b), salinity (c), pH (d), turbidity (e) and dissolved oxygen (f) 
available, as is the case underwater, an augmented TBN with bathymetry and science information may be a promising method for localization and navigation. The utility of TBN for underwater vehicles became valuable with the increase of resolution of bathymetric maps, and the proposed method further refined these maps with the supplementation of more data. Furthermore, the incorporation of science parameter may lead to a low-power and accurate navigation technique for underwater vehicles. 


\section{CHAPTER 5}

\section{GLOBAL LOCALIZATION}

\subsection{Introduction}

The spatiotemporal dynamics of the ocean environment, coupled with limited communication capabilities, make navigation and localization difficult, especially in coastal regions where the majority of interesting phenomena occur. To add to this, the interesting features are themselves spatiotempoally dynamic, and effective sampling requires a good understanding of vehicle localization relative to the sampled feature. Furthermore, these interesting phenomena are usually identified by unique features in the ocean, e.g. significant bathymetric relief, an unstratified water column, or significantly different physical water parameter values. Here, we are interested in the utility of these unique features to aid in localization of underwater vehicles.

Accurate and energy-efficient navigation and localization methods for autonomous underwater vehicles continues to be an active area of research. Since interesting and important ocean processes are spatio-temporally dynamic, their study requires vehicles that can maneuver and sample intelligently while underwater for extended durations. In this chapter, we examine global localization technique that is an improvement of the classic terrain-based navigation technique using physical water data to enhance the utility of traditional maps for navigation and localization. Data from field trials of multiple deployments (See Chapter 2) of an autonomous underwater vehicle (YSI Ecomapper) are used for the analysis and validation. The results show a viable utility of adding water parameters to augment terrain-based maps for the localization of aquatic vehicles in GPS-denied environments.

Underwater vehicles commonly perform underwater navigation via dead reckoning using an accelerometer, magnetometer and depth sensor for feedback. However, these 
instruments are subject to large drift, leading to unbounded uncertainty in location. When confronted with the dynamic environment of the ocean, a state estimate of location can deviate significantly from the actual location; sometimes on the order of kilometers. Two common methods of correcting this issue are 1) surface more frequently for a GPS fix, or 2) integrate more accurate, energy intensive sensors, such as Doppler velocity loggers (DVLs). Both of these methods have drawbacks. Continually surfacing for a GPS fix takes away from sampling time and requires that more energy be used for communications. Surfacing also poses a physical threat to the vehicle, as it might accidentally surface in a hazardous location, e.g. a shipping lane. Using more powerful sensors consumes the finite energy supply of an AUV faster and significantly reduces the deployment duration. To optimize time spent collecting data with these vehicles, it is desirable to find alternative means of reducing position uncertainty while underwater.

Even with higher resolution bathymetric maps, traditional TBN alone can result in significant navigational error, especially in regions of little to no vertical relief. To enhance the ability to navigate and localize, we have developed an augmented TBN that incorporates physical science data, i.e. water parameters such as temperature, salinity, $\mathrm{pH}$, etc., to enhance the topographic map that the vehicle uses to navigate under the traditional TBN framework $\left[\mathrm{RFB}^{+} 17 \mathrm{a}\right]$. In this navigation scheme the bathymetric data are combined with the physical science data to enrich the uniqueness of the underlying terrain map. This method of localization has been evaluated with data gathered at multiple locations in both lake and ocean environments. Results from a deployment in the Big Fisherman's Cove, Santa Catalina Island is presented in $\left[\mathrm{RFB}^{+} 17 \mathrm{a}\right]$; these and other preliminary results from our Augmented Terrain Based Navigation (ATBN) have been promising.

For this study, an exhaustive survey of the region of interest was conducted in each location. The motivation for this analysis is to either a) validate that a previously com- 
puted map is valid for a new deployment, or b) update a previous map for current use. In either case, assuming that an initial and exhaustive survey has been conducted once, we are interested in updating this terrain map to provide maximal utility to underwater vehicles for reducing uncertainty in a navigation solution.

\subsection{Background}

\subsubsection{Localization}

The problem of mobile robot localization can be understood as the process of determining a pose or configuration (position and orientation) of a robot in a known (or unknown) environment, usually using a reference map or a similar representation [LaV06]. Localization is an essential problem in mobile robotics because it precedes other problems e.g., navigation, coverage, mapping, searching, planning, and patrolling. Applications for robots are endless, including precision agriculture, security, surveillance, ocean monitoring and home robotics, industry automation among others. The present research addresses the problem of global localization [TFBD01, FBT99], in which an underwater vehicle has to find its configuration in the aquatic environment without having any information about its initial configuration (in contrast with the tracking problem in the next chapter, where the robot knows its last position). The majority of localization techniques are based on Bayesian filters e.g., particle filters [TFBD01, Die03] or Kalman filters [LDW91a, JLV99a], which are more expensive in terms of computation time and memory, and require sophisticated sensors, infrastructure and motion modeling. The originality of this work is that we rely solely on the bathymetric information and water sensor data collected by the vehicles for solving the problem of localizing a robot in a particular 
environment that is suitable for a device of meager computational ability, potentially even being realized directly in a field programmable gate-array.

The localization problem is challenging to solve and has consequently attracted considerable theoretical attention [OL05, OL07a, EKOL08a, EL13]. The motivation of our work is to use the sensor data that the underwater robot collected as a basis for investigating the intrinsic limits of the localization problem. What is possible here depends on the environment and parameters of the robot sensors, so we explore automated processes to uncover answers to these questions that are given in a particular setting as input.

Several works attempt to localize and navigate AUVs in locations where GPS and DVL are unavailable. In $\left[\mathrm{MRR}^{+} 15\right]$, a drifter was developed to help document changes in sediment runoff from river catchments in Australia. In [SM14], the authors propose a novel AUV localization method using preloaded flow velocity forecast maps and a particle filter based on resemblance between forecasts and local estimation. Their work presents promising results and shows the possibility of performing converging global underwater localization through partial utilization of the background flow information. A work on deep water data sets using the temporal evolution of water currents and employing high fidelity spatial models to account for the horizontal water current field can be seen in [MKE15]. In [SBT ${ }^{+}$17], the authors used an on-board visual relative localization aiming at self-stabilization of multi-MAV groups without GPS. These works show the necessity for robots with limited on-board sensing suites to be able to navigate and localize themselves even in challenging outdoor environments. In [SKS12a], an autonomous glider was used in missions with the objective of localization and navigation using an unscented Kalman filter, which is a Bayesian filtering algorithm used to propagate and update the system state, to estimate the position, altitude and velocity of the vehicle over time.

Three main methodologies have been used for underwater navigation lately: Inertial Navigation, Acoustic Navigation and Geophysical Navigation [MM17]. Nonetheless, it 
is possible to find a combination of these methodologies in the literature with the goal of increasing the robustness of navigation solutions. In the following subsections, an overview of these different methodologies will be presented.

\subsubsection{Inertial Navigation}

The use of inertial navigation is motivated by the short-term accuracy and uses deadreckoning with Inertial Measurement Units (IMUs) for estimating position and velocity. IMUs sensors include accelerometers, gyroscopes, magnetometers and pressure sensors [JSPG09]. Dead-reckoning can be understood as the process of estimate a position of an object based on previously determined positions. It is one of the most used notions for position estimation, having been used by Columbus and other explorers at the end of 15th century. and it is the backbone of the popular Kalman filtering technique, which is still used nowadays. Although inertial navigation is a good solution with short-time accuracy, it tends to drifts over time and quickly becomes infeasible for underwater navigation. Another advantage of inertial navigation is the fact that it does not sends or receives any external signals, which makes it robust and free of interference or jamming [MM17]. It is highly dependent on the accuracy of the sensors and it can drift up to $1.8 \mathrm{~km}$ per day for submarine and spacecraft applications; approximately $1.5 \mathrm{~km}$ per hour for airliners and military aircraft; and over $15 \mathrm{~km}$ per hour and in guided weapons and unmanned aerial vehicles [Gro13] and [MM17]. A development and implementation of a complete model-aided inertial navigation system for underwater vehicles can be found in [HBH08], where a simultaneous estimation of the vehicle model output errors and current aided the position estimate with significantly lower errors than traditional INS throughout time. A comprehensive testing and analysis of a particle filter framework for real-time terrain navigation for underwater vehicles was presented in [Don12] and the results indicated that 
the use of bathymetric information to obtain georeferenced localization for underwater instead of relying on Global Positioning System (GPS) updates or acoustic localization methods.

\subsubsection{Acoustic Navigation}

The fundamental notion of acoustic navigation is the exchange of acoustic signals among beacons and robots with the goal of estimating the robotposition. The most popular ap-

proaches for this sort of navigation are: the Long Baseline (LBL), the Short Baseline (SBL) and the Ultra Short Baseline (USBL) [MM17]. One of the disadvantages USBL is the short-range capability in navigation and high cost. In [WYSH99b], the authors investigated a combination of LBL and inertial navigation with Doppler systems and results showed that this combination outperformed standalone LBL systems in terms of update rate and accuracy and it is a good option for remotely operated underwater vehicles (ROVs). Another combination of inertial and acoustic navigation using ping-response protocol can be found in [CZMF16], where the authors propose a Bayesian near-real time state estimation combining filtering and smoothing, resulting in good accuracy in estimation and capability of being extended to other inertial navigation frameworks. A Networked-Long Base Line System was proposed and developed in [ŚPMD17], where the authors evaluated different communication schemes in the underwater channels and their impact on the acquisition of range measurement, reaching the conclusion that the proposed methodology obtained a reduction on the localization errors of $30 \%$ on average and up to $90 \%$ when compared to respect to Time Division Multiple Access (TDMA). A preliminary study of a combination of underwater communication and navigation of AUVs using an acoustic modem, attitude, and depth sensors, and no Doppler velocity log (DVL), and a surface vehicle with an acoustic modem and GPS is presented in [HW16]. 
A combination of a Strapdown Inertial Navigation System and LBL solving the problem of Fuzzy correlation peak problem for acoustic propagation multipath was presented in $\left[\mathrm{ZSC}^{+} 16\right]$. According to [MM17], the most accurate system is the LBL due to its reliable position estimation that is independent of the operational depths; however, its cost and time to build and remove the infrastructure become a huge drawback for its utilization.

\subsubsection{Geophysical Navigation}

The main idea behind Geophysical Navigation is the use of physical features of the environment so that the autonomous underwater vehicle can use sensor data and a priori environment map to have an accurate estimate of localization. This methodology is the support of this entire research. One of the advantage of a map-based navigation methodology is it on-board capability, where the vehicle can navigate without the need for external devices for accurate estimate of localization. Also, this allows a larger operational range when compared to acoustic navigation systems. The most popular geophysical navigation system is the Terrain-Based Navigation.

\section{Terrain-Based Navigation}

Prior to satellite-based navigation, e.g. GPS, long-distance navigation systems were developed for missiles [Gol80a]. Due to its unique characteristics e.g., robustness against interference or jamming and ability to work regardless of the weather condition and time of the day [MM17]. Data from an embedded altimeter were compared to ground elevations that were provided in a stored map or look-up table. The navigational accuracy of this method is dependent upon the resolution of the underlying topography map and the accuracy of the elevation measurement; both very good for terrestrial applications. This system became redundant after the introduction of GPS, although it is still a useful 
navigational aid for GPS-denied environments, e.g. underwater. A detailed survey of research and current challenges in underwater navigation, summarizing existing work on TBN for underwater vehicles, is provided in [KEW06]. One clearly identified shortcoming of TBN in the aquatic environment is the lack of accurate, high-resolution maps of the sea floor in many regions, which does not invalidate the methodology used. Additionally, sensor limitations, especially the limitations of optical range sensors, substantially restrict TBN underwater. In [KEW06], it is concluded that improved navigation will enable new missions that would previously have been considered infeasible or impractical. Updated bathymetric maps with higher resolution provide motivation for revisiting the application of this method for low-power, accurate navigation underwater, see e.g. [SKS16]. It is also important to highlight that TBN for autonomous underwater vehicles differ from the application by aircrafts due to the vehicles dynamics, sensor capabilities and terrain variability. According to [MM17], an implementation of TBN for aerial vehicles usually needs a combination of sensors such as a barometric altimeter, in order to output the height above the mean sea level (MSL), and radar or laser altimeters, which is required to obtain the height of the vehicle from the terrain. For underwater navigation, on the other hand, the robot needs to accurately know its depth from the water surface and its altitude towards the sea bottom in order to estimate the total water column for a certain area of the aquatic environment. Nonetheless, it is not always straightforward the measurement of the total water column in an aquatic environments, the most used techniques for achieving this bathymetric information is by using expensive but highly-accurate sonar sensors, e.g., Multibeam Echosounders (MBE), Doppler Velocity Logger sonars (DVL) or single beam sonars [MM17].

In [HMH07], the authors explore different methodologies underwater navigation and separates the global search algorithms (geophysical approach) into 3 different categories: the Terrain Contour Matching (TERCOM) [Gol80a], the Point Mass Filter (PMF) and 
the particle filters. The latter has been recently demonstrated by Lagadec [Lag10] to be feasible under ice for long term glider navigation. Lower relief maps of regions above the arctic circle with a resolution of $2 \mathrm{~km}$ were sufficient to navigate with reasonable accuracy ( $\sim 1 \mathrm{~km}$ accuracy, with a mean accuracy of approximately $8 \mathrm{~km}$ in one simulation). The study suggests that for real deployments, technological advances would be necessary to achieve the required navigation performance. However, higher relief bathymetric maps could facilitate the implementation of a TBN that operates online, in real time. The primary limitation of the technique presented in [Lag10] was the lack of an accurate terrain map, which does not invalidate the methodology used. A number of other studies have utilized particle filters as part of a TBN framework for underwater vehicles [Lag10]$\left[\mathrm{GGB}^{+} 02\right]$. The main problem with current implementations of TBN frameworks has been regarded in [HMH07] to be the convergence problems in terrain with little variation.

\subsubsection{Improving TBN}

Improving TBN ultimately requires a higher resolution in the underlying map. To do this, we propose a method for creating an augmented terrain map that combines both bathymetric information and physical water-parameter data. Initial results of this method are presented in $\left[\mathrm{RFB}^{+} 17 \mathrm{a}\right]$. The assumption that this augmentation provides a reliable model comes from the concept of Environmental or Ecological Niche Models; mapping species distribution over geographic ranges to create predictive models that infer where various species are likely to be found [MM15]-[MTMT09]. By monitoring specific physical parameters of an environment and understanding the tolerances of a certain species, it is possible to model where that species will most likely be present [MTMT09]-[Pet06]. Here, we hypothesize that these niches may also be utilized for underwater vehicle navigation. 


\subsection{METHODS}

To extend autonomy in the underwater domain, we developed an augmented TBN that incorporates physical science data, i.e., water parameters such as temperature, salinity, $\mathrm{pH}$, etc., to enhance the topographic map that the vehicle uses to navigate under the traditional TBN framework $\left[\mathrm{RFB}^{+} 17 \mathrm{a}\right]$. In this navigation scheme the bathymetry data are combined with the physical science data to enrich the uniqueness of the underlying terrain map.

\subsection{Global Localization Within the Terrain Map}

For our simulation, we assume that the vehicle follows a nominal, bicycle-type trajectory, and that the vehicle's angular rotation rate and linear acceleration are driven by white, zero-mean Gaussian noise processes represented by the vectors $\eta_{q}(t)$ and $\eta_{v}(t)$, with covariance matrices $\mathbf{Q}_{q}$ and $\mathbf{Q}_{v}$, respectively. The system state evolves in continuous time according to

Given different terrain-based maps using just the bathymetric parameter and a combination of bathymetric information and the aforementioned water parameters, we test the localization problem. It is important to highlight that the augmented terrain map looks similar to the scalar field of the depth parameter; however, the augmented one has a lower global correlation, making the localization of a trajectory converge faster. Localization is known as the central problem in mobile robotics and can be understood as the task of systematically eliminating uncertainty in the pose of a robot [OL07b]. Suppose an underwater robot is given a map of the ocean environment. The goal of the AUV is to move within that environment, collecting information about the water parameters, e.g., temperature, salinity, dissolved oxygen, etc., combine these parameters using the weights calculated in subsection 5.3, and eliminate uncertainty about where it is located in the 
map. More specifically, one terrain map was created for the first day of deployment using depth information combined with the water parameters. Another terrain map was created for the second day using depth information combined with the water parameters. Another deployment of the AUV on the second day is performed and we extract a determined path to be localized. Can this path be localized in the terrain map created for the same day (day 2)? What is the average error? Can it be localized in the terrain map created for the day before (day 1)? What is the average error? Algorithm 3 returns the final tree created with the localized path in the terrain map.

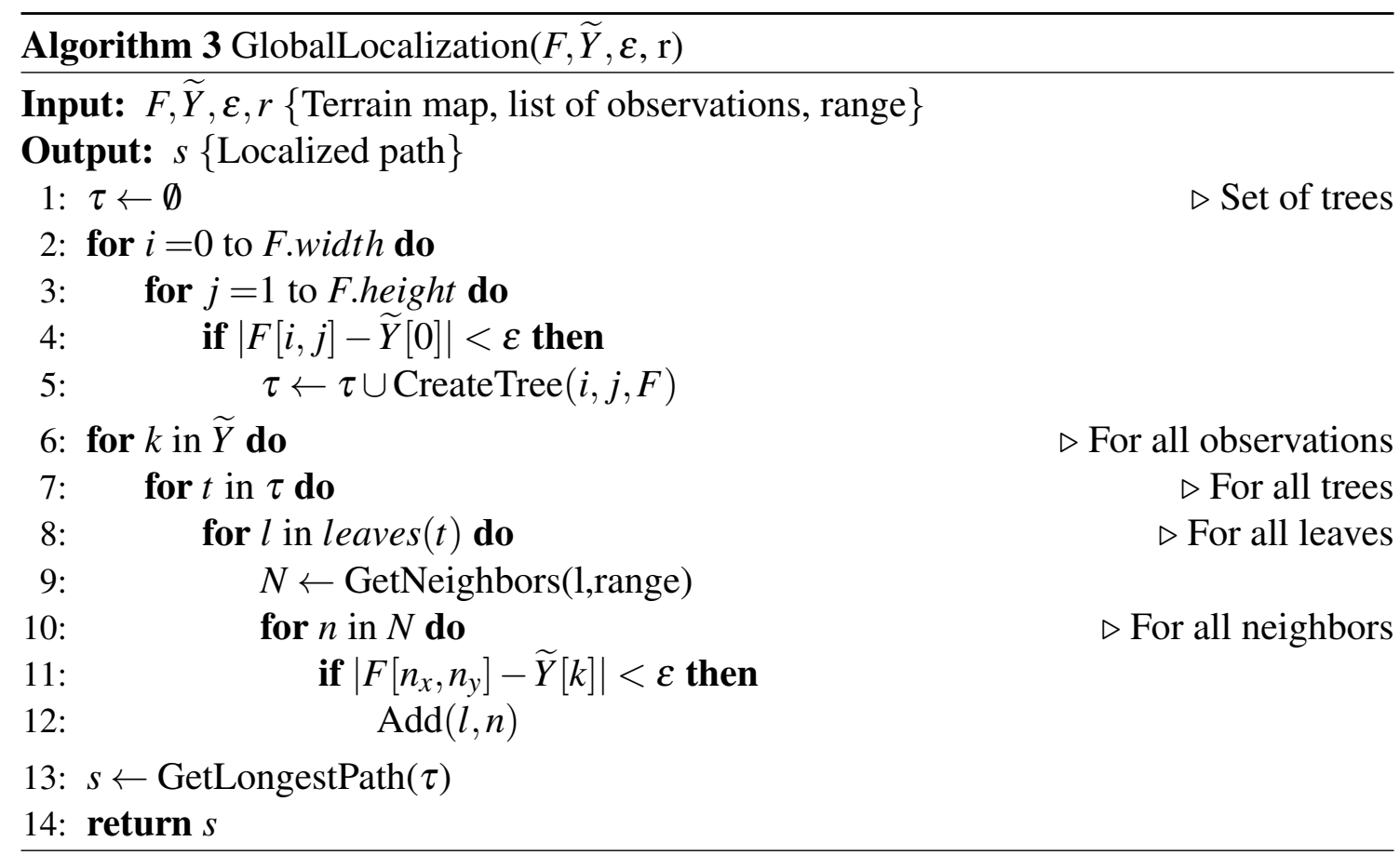

This algorithm searches for an approximate path and has the following inputs: the terrain map, a list of observations from the path to be localized, an epsilon defining a margin of tolerance where no penalty is given to errors in localization and a range representing the how far an observation can be localized following another. The output is the coordinates in the terrain map where the observations were localized for a certain path. The algorithm starts with an empty set of trees (line 1). For all coordinates $(x, y)$ 
in the map, possible candidates are found for the first observation $\widetilde{Y}_{0}$, thereby initializing the root of the trees (lines 2-5). Each tree represent one possible location for the path. For each remaining observation, we iterate over the each leaf of each tree, trying to find a neighbor to be added to the tree. A neighbor is within range $r$ and with a margin of tolerance $\varepsilon$ (lines 6-12). The deepest tree contains the coordinates of the localized path in that map. The execution time increases with the number of observations to be localized and decreases with a low global correlation hypothesis described in section II-C.

\subsubsection{Localization Results}

Regarding the localization problem, tests were executed on the same day and on different days and errors in localization were calculated using geographic information system techniques on the bay off of Santa Catalina Island. Tables 5.1 and 5.2 show the statistics of the parameters used in the localization tests for the two-day surveys. The terrain maps for both days were created using the methodology proposed in Section 5.3-B and the localization algorithm proposed in Section 5.3-C. In Fig 5.1, the path in red is the target location where the vehicle performed a second survey (the first is the survey which the terrain maps were developed upon). The path in yellow is the approximate location using the combination of water parameters with bathymetric information. The average error in localization is $4.0064 \mathrm{~m}$.

In Fig 5.2, the path in red color is the target location where the vehicle performed a second survey on a different day, whereas the path in black color is the approximate localized trajectory using the combination of water parameters with bathymetric information. The average error in localization is $29.86 \mathrm{~m}$. This localization algorithm is suitable as a solution to the TBN problem because it naturally incorporates the property that the longer a path is traversed, the more likely a single solution will emerge. 
Table 5.1: Minimum, maximum, mean and standard deviation of the water parameters considered for the Santa Catalina Island deployment on the first day.

\begin{tabular}{|l|c|c|c|c|}
\cline { 2 - 5 } \multicolumn{1}{c|}{} & Min & Max & Mean & Std \\
\hline Latitude & 33.444 & 33.446 & - & - \\
\hline Longitude & -118.49 & -18.48 & - & - \\
\hline Depth & 0.31 & 35.03 & 13.318 & 7.2885 \\
\hline Dissolved Oxygen & 8.12 & 9.36 & 8.4818 & 0.29481 \\
\hline Turbidity & 0 & 3.7 & 1.3201 & 0.47162 \\
\hline Salinity & 33.77 & 33.95 & 33.861 & 0.47162 \\
\hline Temperature & 21.7 & 23.2 & 22.346 & 0.028379 \\
\hline pH & 8.29 & 8.49 & 8.3945 & 0.41324 \\
\hline Conductivity & 51.33 & 51.59 & 51.461 & 0.038991 \\
\hline
\end{tabular}

Table 5.2: Minimum, maximum, mean and standard deviation of the water parameters considered for the Santa Catalina Island deployment on the second day.

\begin{tabular}{|l|c|c|c|c|}
\cline { 2 - 5 } \multicolumn{1}{c|}{} & Min & Max & Mean & Std \\
\hline Latitude & 33.444 & 33.445 & - & - \\
\hline Longitude & -118.49 & -18.48 & - & - \\
\hline Depth & 0.87 & 11.93 & 4.6293 & 1.6659 \\
\hline Dissolved Oxygen & 8.17 & 9.78 & 8.6889 & 0.35432 \\
\hline Turbidity & 0 & 4.7 & 1.3017 & 0.48395 \\
\hline Salinity & 33.66 & 34.02 & 33.864 & 0.48395 \\
\hline Temperature & 21.89 & 22.9 & 22.381 & 0.023499 \\
\hline pH & 8.34 & 8.47 & 8.4038 & 0.22132 \\
\hline Conductivity & 51.19 & 51.68 & 51.466 & 0.028323 \\
\hline
\end{tabular}

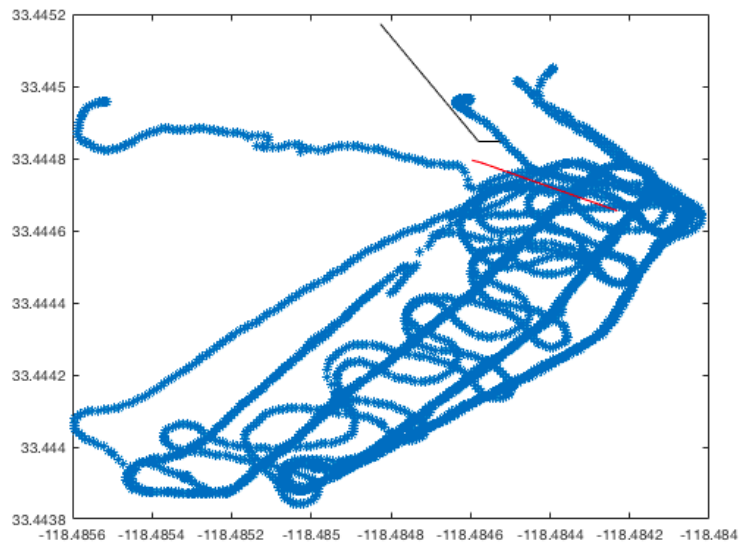

Figure 5.1: The underlying scalar field computed from the data gathered during a dense lawnmower path in the Big Fisherman's Cove off Santa Catalina Island, CA. 


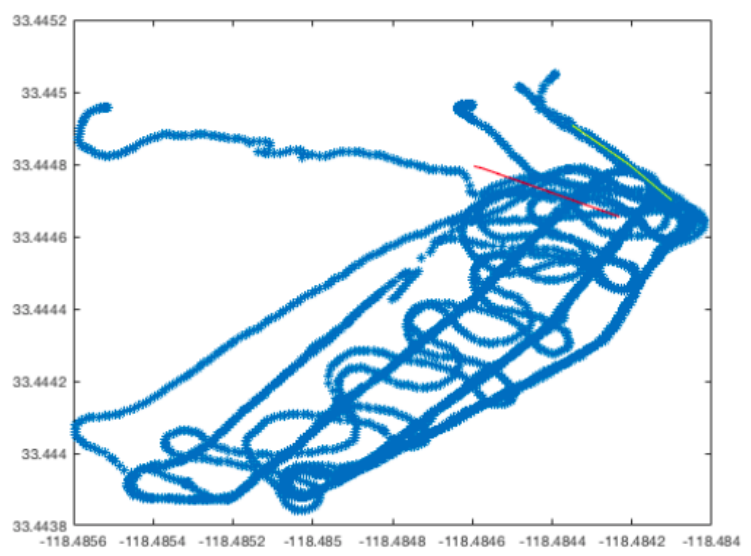

Figure 5.2: The underlying scalar field computed from the data gathered during a dense lawnmower path in the Big Fisherman's Cove off Santa Catalina Island, CA.

Another localization test was done on the first day only, where a segment of a trajectory performed by the AUV was extracted in order to be localized on the map created with a combination of water parameters and bathymetric information. The weighting scheme outputted the following weights for their respective parameters: 0.9402 (depth), 0.0493 (ODO), $0.0021(\mathrm{pH}), 0.0025$ (salinity), 0.0019 (temperature) and 0.0040 (turbidity). It is important to highlight that the data collected by the AUV in this localization problem was not used to create the underlying terrain maps. The black segment in Fig 5.4 represents the original trajectory to be localized and the red segment represents the approximate localized trajectory with an average error of $0.5445 \mathrm{~m}$.

Another localization experiment in the Big Fisherman's Cove, at Santa Catalina Island, CA, USA, can be seen in Figure 5.4, where the orange segment represents the original trajectory, executed during the same survey (in blue) from which the reference map was created; the green segment represents the approximate trajectory localized by traditional terrain-based navigation systems; and the red trajectory represents the approximate trajectory localized by the augmented terrain-based navigation framework developed and described in this chapter. By using the traditional terrain-based navigation approach with only bathymetric information, a $9.5031 \mathrm{~m}$ average error in localization was achieved, 
Table 5.3: Weights for the water parameters for two different missions in two different days in Santa Catalina Island, CA, USA

\begin{tabular}{|l|l|l|l|l|l|l|}
\cline { 2 - 7 } \multicolumn{1}{c|}{} & Depth $(\mathbf{m})$ & Temp $\left({ }^{\circ} \mathbf{C}\right)$ & Sal $(\mathbf{p p t})$ & pH & Turb $(\mathbf{N T U})$ & ODO $(\mathbf{m g} / L)$ \\
\hline Day 1 & 0.5611 & 0.0829 & 0.0760 & 0.0948 & 0.09813 & 0.0869 \\
\hline Day 2 & 0.5981 & 0.0804 & 0.0863 & 0.0311 & 0.1108 & 0.0930 \\
\hline
\end{tabular}

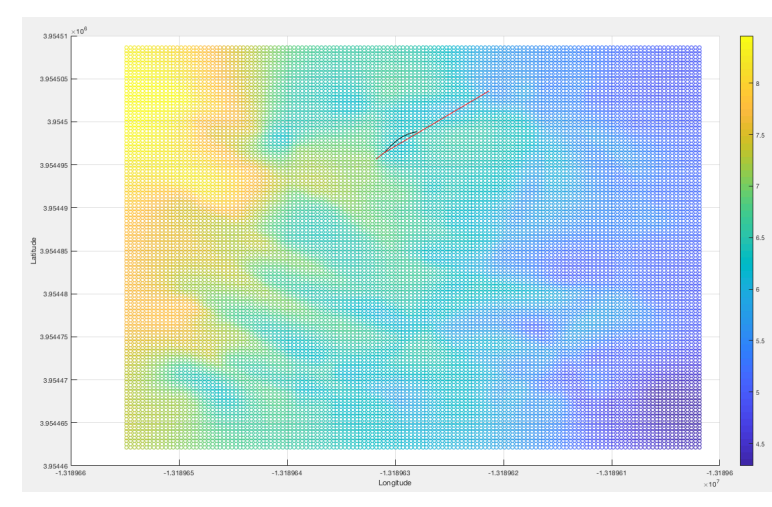

Figure 5.3: Localization of a segment of a trajectory on the first day of deployment in the Big Fisherman's Cove off Santa Catalina Island, CA. The black segment represents the original trajectory and the red segment represents the approximate trajectory localized by the proposed methods. The colors in the plot varies according to the combination of water parameters and bathymetric information. The $x$-axis is longitude and the $y$-axis is latitude.

whereas the presented augmented TBN achieved $8.6631 \mathrm{~m}$ average error. Another test was executed where a trajectory from the second day of deployment was localized using the map created for the first day of deployment. In this experiment, traditional TBN had $21.3511 \mathrm{~m}$ average error in localization, whereas the presented augmented TBN achieved $21.2432 \mathrm{~m}$ average error. It is important to highlight that the not only the accuracy was lower but also the number of candidates considered in the localization algorithm was less in the ATBN than in the traditional TBN, making localization faster and more effective.

The weighting scheme outputted the following weights for their respective parameters can be seen in Table 5.3. Localization results using our proposed ATBN maps have provided promising results that reduce navigation uncertainty as compared with dead reckoning and traditional TBN results. 


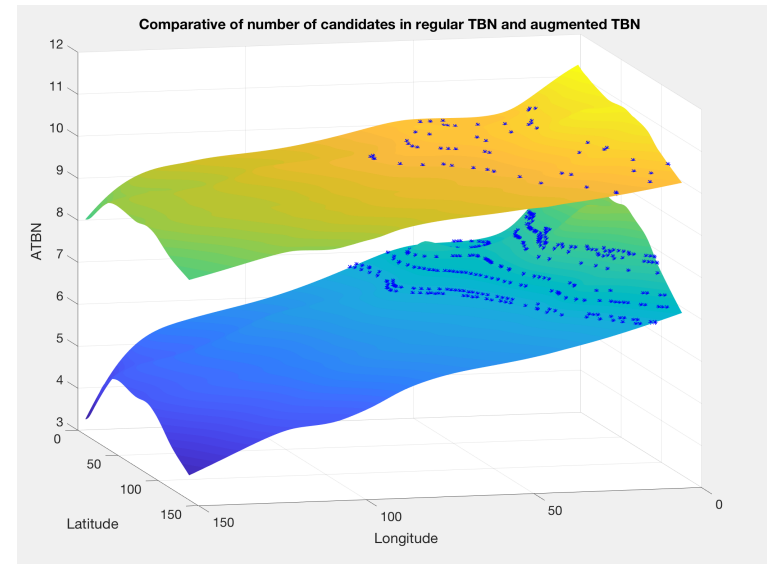

Figure 5.5: A bathymetric map (bottom) used for regular terrain-based navigation approaches and a combination of bathymetric information and water parameters (top) are placed together with the objective to compare the number of candidates for the same sensor reading. When just depth is analyzed, there are more candidates per observation than when the combination of bathymetric information and water data are combined.

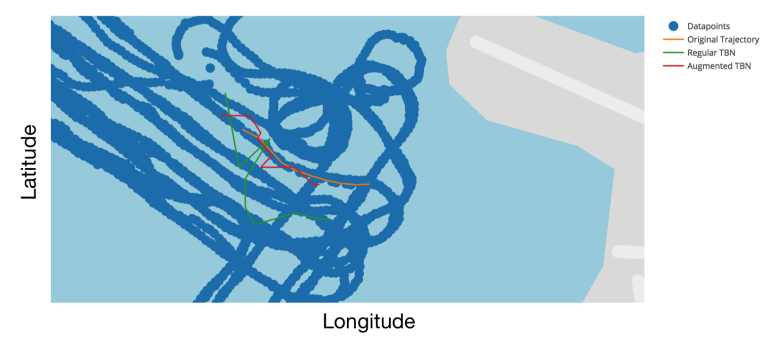

Figure 5.4: Localization of a trajectory from a mission on the first day of deployment at the Big Fisherman's Cove off Santa Catalina Island, CA. The orange segment represents the original trajectory, executed during the same survey (in blue) from which the reference map was created; the green segment represents the approximate trajectory localized by traditional terrain-based navigation systems; and the red trajectory represents the approximate trajectory localized by the augmented terrain-based navigation framework. The $\mathrm{x}$-axis is longitude and the $\mathrm{y}$-axis is latitude.

\subsection{CONCLUSIONS AND FUTURE WORK}

We presented a methodology to augment terrain-based navigation methods by combining bathymetric data with physical water parameter data. This proposed method generates a scalar field that can be used as a terrain map with standard TBN algorithms. We proposed 
and implemented a global localization algorithm to localize a list of observations collected by the underwater vehicle in the region where the terrain map was created. We have tested this methodology on multiple datasets from field trials, and preliminary results imply that our methods can increase accuracy in navigation solutions.

All of the sampled environments are similar because they are shallow waters with organic substrate close to the surface. It is clear from the methodology of computing the weights for the individual parameters that the data with the highest variability across the surveyed region receives the highest weight. Further research in this computational process is warranted for extending this technique. 


\section{CHAPTER 6}

\section{TRACKING}

\subsection{Overview}

This chapter presents a tracking algorithm for Autonomous Underwater Vehicles (AUVs) using a whitening approach. Localization of underwater vehicles faces challenges that span from the spatiotemporal aspects of the properties of the water to unavailability of GPS signal. To overcome some of these problems, bathymetric information has been used to estimate the state trajectory of AUVs but it has limitations in regions of little to no vertical relief. To address this issue, we developed a new method for localizing the state trajectory of an AUV by considering not only the bathymetric information but also sensor data e.g., dissolved oxygen concentration, temperature, and turbidity. Nonetheless, the correlation of these parameters masks the true distances between data points. Therefore, we decorrelate the sensed data using a zero-phase components analysis, also known as Mahalanobis whitening; thus, revealing the true distances between data-points and finally being able to localize with higher precision. Results are shown for deployments carried out on two consecutive days in the Big Fisherman's Cove on Santa Catalina Island, CA, USA. Tests were performed for each day where a new list of sensor data observations (without GPS information) is localized based on the depth, turbidity and dissolved oxygen values of the historical data. Using GPS information as a measurement of the errors in localization, a median of 4.79 meters was obtained when tracking was performed on correlated data and a median of 1.16 meters was obtained when tracking was performed on the whitened data. Results indicated that ZCA Mahalanobis whitening is a promising methodology to aid navigation of AUVs in GPS-denied environments.

Localization and tracking of Autonomous Underwater Vehicles (AUVs) can become compromised by the spatiotemporal dynamics of the ocean environment and the lim- 
ited communication capabilities. Our work focuses on the coastal regions of the ocean due to the higher frequency of occurrence of interesting phenomena, e.g., cyanobacterial blooms. Furthermore, the interesting features are themselves spatiotemporally dynamic, and effective sampling requires a good understanding of vehicle tracking relative to the sampled feature. These interesting phenomena are usually identified by unique features in the ocean, e.g., significant bathymetric relief, an unstratified water column, or significantly different physical water parameter values. Here, we are interested in the utility of these unique features to aid in localization of underwater vehicles.

\subsection{Methodology}

This method of tracking has been evaluated with data gathered in two deployments performed in the Big Fisherman's Cove, Santa Catalina Island, CA, USA, for two consecutive days, from 4:27 to 8:24 pm and 6:11 to 8:19 pm (MT), respectively (Fig. 6.1). After processing the data (e.g., outliers detection), the data passes through a whitening process step, which is a statistical analysis to transform random variables to orthogonality. Multiple possible whitening procedures, e.g., principal component analysis (PCA), Cholesky matrix decomposition and zero-phase component analysis (ZCA) are discussed and presented in [KLS18], with results showing that the ZCA-cor whitening process is capable of producing sphered variables that are maximally similar to the original variables. The purpose of whitening the data before using it for localization and tracking comes from the fact that the Euclidean distance between two data-points are possibly affected by their correlation. Therefore, we need to capture the true statistical distances between any two data-points for a good approximation in localization. 


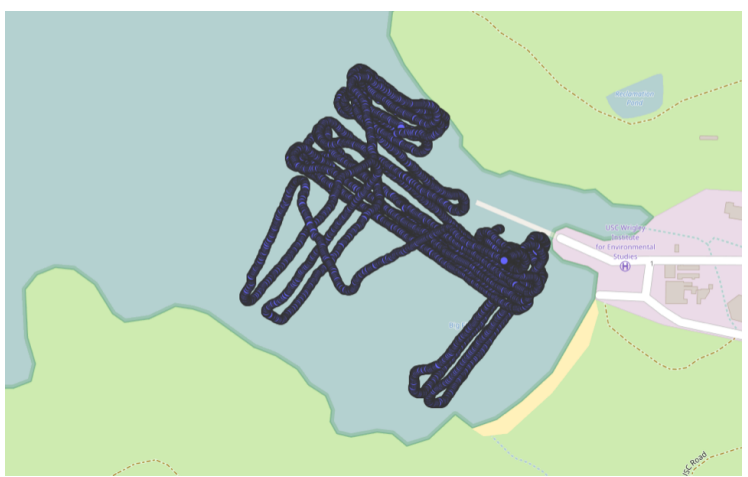

(a)

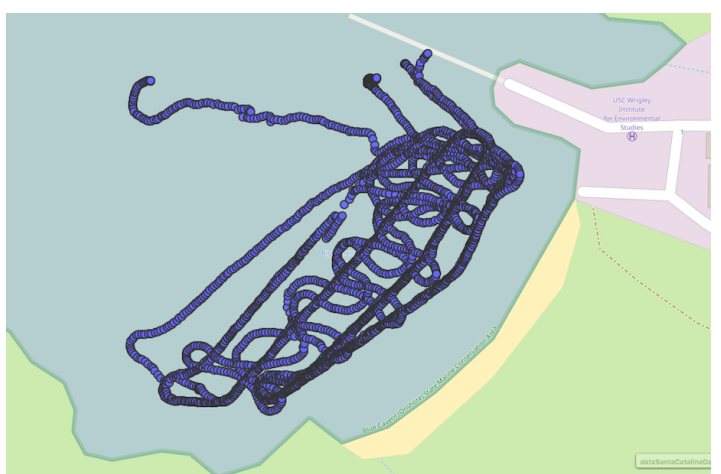

(b)

Figure 6.1: This image shows the region of data gathering during a dense lawnmower path in the Big Fisherman's Cove off Santa Catalina Island, CA, on the first (a) and second (b) days.

A tracking algorithm is presented by using Sensor data collected by an Automated Underwater Vehicle (AUV). We use a whitening linear transformation to disperse the data and using Euclidean distance we can determine good candidates for the vehicle's position once it loses GPS signal. The effect of the whitening linear transformation is equivalent to using the Mahalanobis distance, which takes into account the effect of the correlation between the random variables measurements obtained from the sensors. This allows us to track the position of the vehicle within small intervals of time (less than an hour) until the GPS signal returns.

Terrain-based navigation frameworks have been used to localize AUVs, but it requires a high resolution of bathymetric maps. Nonetheless, the navigational error can still occur, especially in regions of little to no vertical relief. Based on the incorporation of physical science data, i.e., water parameters such as temperature, salinity, $\mathrm{pH}$, etc., to enhance the topographic map that the vehicle uses to navigate under the traditional TBN framework, as shown in $\left[\mathrm{RFB}^{+} 17 \mathrm{~b}\right]$, our approach used bathymetric information and water data to improve the localization and tracking. 
The contribution of this work is to present a novel application of the ZCA Mahalanobis whitening for decorrelating of physical science data of the water and bathymetric maps in order to aid tracking of underwater vehicles when GPS signal is lost. We implemented a novel tracking algorithm for underwater vehicles in GPS-denied environments and performed simulation tests.

The remainder of the paper is structured as follows. Section 6.3 outlines the related work from the literature. In Section 6.4, we model our workspace, robot, and observation space. Also, we formulate the problem we consider in the same section. We describe our step-wise methodology in Section 6.5. Section 6.5.6 illustrates the simulation results to validate our algorithm. Finally, we conclude with the discussion and future work in Section 6.5.8.

\subsection{Related Work}

\subsubsection{Robot Localization and Tracking}

The typical robot localization methods use recursive Bayesian filters such as particle filters [TFBD01, Fox03] and Kalman filters [LDW91b, JLV99b] making use of the data from range and camera sensors. However, these methods are computationally expensive and require a large memory capacity. Also, localization methods with limited sensing are proposed for mobile robots [OL07b, ABS18, EKOL08b], in which the robots entail less memory and computation to solve the localization task. The authors of this stream of research consider the motion model of simple robots whereas we do not take the dynamics of the robot into account in this work. Instead, we consider the historical sensor data of the underwater vehicles and infer the location of the deployed robot based on these data. There is a subtle distinction between localizing a robot and tracking a robot. In tracking a 
robot, the initial robot state is known. In localizing a robot globally, the initial state of the robot is unknown which is also called global localization. Some global robot localization approaches are presented [TFBD01, FBT99, ABS18], where a robot has to find its state in the whole environment without having any information about its initial state. Closely related position tracking methods for robots and targets from their initial positions are proposed in [BFH97, TIF14, TBVHI13].

\subsubsection{Terrain-Based Navigation}

Before the use of satellite-based navigation, such as GPS, long-distance navigation frameworks were developed for missiles [Gol80b]. Data from an embedded altimeter were compared to ground elevations that were provided in a stored map or look-up table. The navigational accuracy of this method is dependent upon the resolution of the underlying topography map and the accuracy of the elevation measurement; both very good for terrestrial applications. This system became redundant after the introduction of GPS, although it is still a useful navigational aid for GPS-denied environments, e.g., underwater. A detailed survey of research and current challenges in underwater navigation, summarizing existing work on TBN for underwater vehicles, is provided in [KEW06]. One clearly identified shortcoming of TBN in the aquatic environment is the lack of accurate, high-resolution maps of the seafloor in many regions, which does not invalidate the methodology used. Additionally, sensor limitations, especially the limitations of optical range sensors, substantially restrict TBN underwater. In [KEW06], it is concluded that improved navigation will enable new missions that would previously have been considered infeasible or impractical. Updated bathymetric maps with higher resolution provide motivation for revisiting the application of this method for low-power, accurate navigation underwater, see, e.g., [SKS16]. 


\subsubsection{Data Whitening}

Whitening and decorrelation has been widely used in data-driven applications, e.g., data compression, improve the feature decorrelation for Hidden Markov Models-based speech recognition [DDCW98], improving pedestrian detection [NDH14], optimization of shiftinvariant filters for spatio-chromatic image [BSF11], reduce the subspace in which beamforming algorithms operate [KRW09], among others. In [HMR12], the authors show how whitened features are better for computing similarities in clustering and classification than non-whitened features.

\subsection{Preliminaries}

\subsubsection{Model Definition}

We consider a small oceanic environment as our workspace. This workspace, denoted by $\mathscr{W} \subset \mathbb{R}^{2}$, is modeled as a $2 \mathrm{D}$ polygonal environment. An autonomous underwater vehicle $\mathscr{A}$ is modeled as a point robot without considering its orientation. The state space of the vehicle is represented as $X=\mathscr{W}$ which consists of all navigable locations of the environment. Each state of the robot $x$ denotes a geographic coordinate in the form of longitude and latitude. A state trajectory of the vehicle is denoted as $\tilde{x}:[0, t] \rightarrow X$ for a finite time interval $[0, t]$. We assume that the vehicle has $m$ sensors for the observation of the environment. Let $Y \subset \mathbb{R}^{m}$ be the observation space, which is the set of $m$ sensor output values. An observation history for the robot is defined as $\tilde{y}:[0, t] \rightarrow Y$. Suppose a sensor $h: X \rightarrow Y$ is given and is applied over an interval of time $[0, t]$. For every $t^{\prime} \in[0, t]$, some observations $\tilde{y}\left(t^{\prime}\right)=h\left(\tilde{x}\left(t^{\prime}\right)\right)$ are obtained. We define the sensor mapping over $[0, t]$ as $\left[\mathrm{L}^{+} 12\right]$ :

$$
H: \tilde{X} \rightarrow \tilde{Y}
$$


in which $\tilde{X}$ is the set of all state trajectories and $\tilde{Y}$ is the set of all possible observation histories.

In our problem a state $x$ represents GPS data of the underwater vehicle which is defined as:

$$
x=\left[\begin{array}{l}
x_{1} \\
x_{2}
\end{array}\right],
$$

where $x_{1}$ and $x_{2}$ are the longitude and latitude measured by the GPS. We measure $m$ sensor variables that include water column depth, turbidity, salinity, temperature, $\mathrm{pH}$, dissolved oxygen, and conductivity. This multivariable observation can be written as:

$$
y=\left[\begin{array}{c}
y_{1} \\
y_{2} \\
\vdots \\
y_{m}
\end{array}\right],
$$

where $y_{i}$ is the $i$-th coordinate of this observation.

\subsubsection{Problem Formulation}

We have a set of historical data that gives us the state trajectories of the vehicle $\tilde{x}$ and the corresponding sensor observation histories $\tilde{y}$. In a new deployment in the same oceanic environment, the vehicle collects the sensor observation data which is denoted as $\tilde{y}_{\text {new }}$. The vehicle can get lost due to the unavailability of GPS data. Then, our goal is to track the state trajectory of the vehicle right after the GPS signal is lost and we do not receive the GPS data. Let the new state trajectory of the vehicle after the deployment be $\tilde{x}_{\text {new }}$. In this context, we formulate our problem as follows:

\section{Problem 1. Tracking the state of an underwater vehicle:}


Given a state trajectory of a vehicle $\tilde{x}$ and its observation history $\tilde{y}$ for a specific time period, attempt to find the state trajectory of the vehicle $\tilde{x}_{n e w}$ from the new observation history $\tilde{y}_{\text {new }}$.

We have a set of historical data collected in bodies of water within a time range that will leave the analysis of changes in a temporal dimension for later discussion (2 hour range in the collected data set). We will call this historical data set, our "map", and we will assume it looks like a gridded polygon, not necessarily convex, that is, we have lines of sampled data that go from North to South, and lines from West to East, whose endpoints are on the boundary of this polygon; call them grid lines.

The vehicle is deployed and it transmits data which include gps plus some sensor data.

This data is fed to the map, until the vehicle's gps signal is lost, and the tracking process starts with the last gps+sensor data point received which we will call the "source" $S$.

We will call the non-gps data points received after the source, our observation points " $o_{i}$ ", $i>0$. The points $\mathbf{x}_{\mathbf{i}}$ in our map are of the form:

$$
\mathbf{x}_{\mathbf{i}}=\left[\begin{array}{c}
x_{1} \\
x_{2} \\
\vdots \\
x_{n}
\end{array}\right]
$$

where $x_{1}$ and $x_{2}$ are the longitude and latitude measured by the gps, and $x_{i}$, for $i>2$, is one of the non-gps sensored data parameters such as Water column depth (m), Turbidity (Nephelometric Turbidity Units) and Disolved Oxigen (mg/L). Similarly, for the observations $o_{i}$, with the only difference that they do not contain the first two gps parameters Longitude and Latitude, $p_{1}$ and $p_{2}$, respectively.

The goal is to track the motion of this AUV right after the gps signal is lost. At that point, we receive observation points $o_{i}$ missing the gps data. 
So, our approach is to track the vehicle finding the point in our map that statistically closest to the first observation $o_{1}$ lying inside an epsilon ball around the source. This epsilon is approximated by using one of the non-gps parameters that measures the speed of the vehicle and the time elapsed between the source point and $o_{1}$. If no point is found within such epsilon neighborhood, we expand epsilon until our first candidate $c_{1}$ appears in the map. Based on one of our original assumptions, this candidate would appear in the first few expansions from the source. Now notice that these candidates are located either in one of these gridlines or at one of the points during the current deployment that were added to the map until the gps signal was lost. So, we approximate $o_{1}$ with $c_{1}$, and when we receive the second observation $o_{2}$, we will repeat the process by localizing $o_{2}$ inside a new epsilon ball around $c_{1}$, where epsilon is determined by the average speed between $o_{1}$ and $o_{2}$. We will bound this epsilon by some fixed quantity such that if no candidates are within it after having expanded it a few times, we will ignore such observation and wait for the next one to be approximated. Now this statistical distance we mentioned before that is calculated, for instance, between $o_{1}$ and all the points from our map, is done using the Mahalanobis distance, which is equivalent to applying a whitening linear transformation to $o_{1}$ and to all points in our map, and then computing the euclidean distance between them. The advantage of using this distance is that it reverses the effect of the correlation within the parameters used to calculate it; thus revealing the true statistical distance between the sampled points and the observations. As we receive and approximate these observation points with candidates from our map, a curve connecting them is interpolated to describe an approximate path followed by the vehicle. And we continue this process until needed or until the gps signal returns. 


\subsection{Methods}

\subsubsection{Data Acquisition}

The proposed methodology was tested with data from multiple deployments at the Big Fisherman's Cove on Santa Catalina Island, CA, USA and at the Lake Nighthorse, CO, USA. The missions were executed using a YSI EcoMapper Autonomous Underwater Vehicle (AUV) [ysi17] shown in Chapter 2. The AUV was operated on the surface. The range of science data that were collected by the vehicle include Water Column depth (m), salinity (ppt), temperature $\left({ }^{\circ} \mathrm{C}\right), \mathrm{pH}$, turbidity (NTU) and dissolved oxygen $(\mathrm{mg} / \mathrm{L})$.

\subsubsection{Data Preprocessing}

From the original eight sensed quantities in each $y$, we selected Water Column depth (m), turbidity (NTU), and dissolved oxygen (mg/L) using the three largest eigenvalues of the sample covariance matrix of our dataset (Principal Component Analysis). This data was processed to remove outliers, and each $y$ or observation is a triple, that is $m=3$, where each of these sensed quantities is one of its features. The data was also centered such that the mean of each feature is zero.

\subsubsection{Decorrelation Process}

Our process of tracking uses the distance between newly sensed observations $y_{\text {new }}$ and our historical observations $y$ to determine the closest geographical location $x$ of $y_{\text {new }}$. The problem with correlated data is that points that are geometrically close might not be statistically close. 
For instance, in $\mathbb{R}^{2}$, data with correlated features $y_{1}$ and $y_{2}$ would be displayed as in Fig. 6.2.

We have marked two data points with a red and a green cross, and the multivariate mean with the red circles. We chose those two points because they seem to be at the same distance from the mean. However, our intuition should tell us the point marked with red must be further away from our data than the green one is; you can see that the green point is surrounded by many other data points unlike the black one, which is in the boundary of the cluster. If we were to use just Euclidean distance, we would have those two points as being equidistant from the mean, which is deceiving.

The correlation between features show that the data varies in directions different to those of the standard basis of $\mathbb{R}^{n}$. In the covariance matrix of our sampled data set, non-zeros in the non-diagonal reveal these correlations and thus the observations are distributed along some oblique lines which may not even be orthogonal in $\mathbb{R}^{n}$. Therefore, we transform the geometry of that space by changing from the standard basis of $\mathbb{R}^{n}$ to a basis of the eigenvectors of the covariance matrix of the data set with the objective of rotating and rescaling its points along these new axes.

After identifying the axes where the data varies, we rotate the axes to match those eigenvectors. 


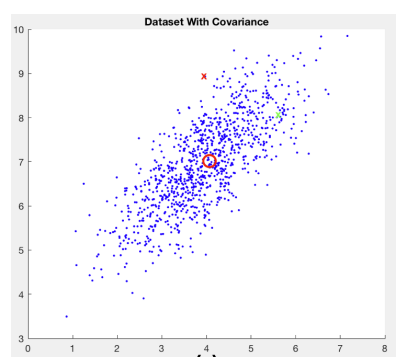

(a)

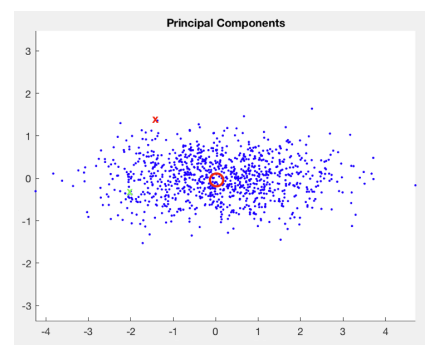

(c)

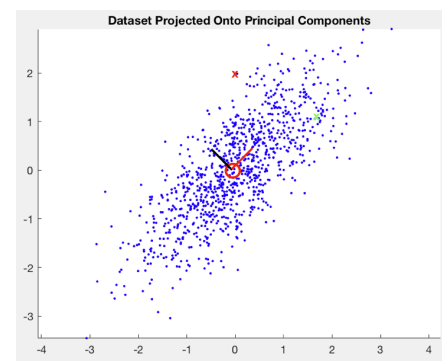

(b)

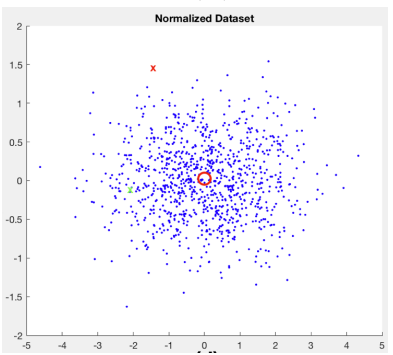

(d)

Figure 6.2: In (a), the correlated data is shown as blue dots and realize that the red and green crosses seem to have similar distances from the multivariate mean. The principal components are shown in (b) as black and red arrows (principal components are orthogonal to each other). In (c) data is projected onto the direction of the first principal component (rotation of the data). The data points are uncorrelated and normalized in this new space as shown in $(d)$.

Following this rotation, we scale the new axes using the variance in the direction of each new axes by using their corresponding eigenvalues obtained from the data covariance matrix.

It is clear now that, statistically, the red point is actually further away from the mean than the green one. At this point, the parameters have been decorrelated.

\subsubsection{ZCA Mahalanobis}

In order to decorrelate the data, we will use a whitening transformation [KLS18]. Whitening consists of applying a linear transformation $\mathbf{W}$ to an $n$-dimensional random data vector $\mathbf{y}=\left(y_{1}, y_{2}, \ldots, y_{n}\right)^{T}$ with mean $E(y)=\mu=\left(\mu_{1}, \mu_{2}, \ldots, \mu_{n}\right)^{T}$ and positive definite $n \times n$ 
covariance matrix (we will call it covariance from now on) $\operatorname{cov}(y, y)=\operatorname{var}(y)=\mathbf{m}$, such that the covariance of the new vector $\mathbf{z}=W y$ of the same dimension $n$ is the $n \times n$ identity matrix $\mathbf{I}$.

Thus, by definition, the vector $z$ will be both standardized and its parameters will have no correlation; in other words, we need to find $W$ such that the following equation holds:

$$
I=\operatorname{cov}(z, z)=\operatorname{cov}(W y, W y)=W \operatorname{cov}(y, y) W^{T}
$$

which implies that

$$
I=W \Sigma W^{T}
$$

And so, this would be equivalent to:

$$
I W=\left(W \Sigma W^{T}\right) W=W\left(\Sigma W^{T} W\right)=W
$$

Hence, we need to find a transformation $\mathbf{W}$ such that $W\left(\Sigma W^{T} W\right)=W$, which holds if and only if

$$
W^{T} W=\Sigma^{-1}
$$

Recall that a square matrix $Q$ is orthogonal if and only $Q^{T} Q=I=Q Q^{T}$, and that if $\Sigma$ is symmetric then it is invertible with positive real eigenvalues and symmetric inverse $\left(\Sigma^{-1}\right)^{T}=\Sigma^{-1}$. What's more, $\Sigma$ being diagonalizable, makes its square root $\Sigma^{1 / 2}$ symmetric too, i.e.,,$\left(\Sigma^{1 / 2}\right)^{T}=\Sigma^{1 / 2}$

Notice that $W=\Sigma^{-1 / 2}$ will satisfy the equation above. This whitening linear transformation, call it $W^{Z C A}=\Sigma^{-1 / 2}$, is optimal in the sense that it guarantees a solution to the least squared distance problem between the original vectors and the transformed ones. For details, please refer to [KLS18]. 


$$
\begin{gathered}
W^{T} W=\left(\left(Q \Sigma^{-1 / 2}\right)^{T}\right)\left(Q \Sigma^{-1 / 2}\right)= \\
\left(\left(\Sigma^{-1 / 2}\right)^{T} Q^{T}\right)\left(Q \Sigma^{-1 / 2}\right)= \\
\left(\Sigma^{-1 / 2}\right)^{T} Q^{T} Q \Sigma^{-1 / 2}= \\
\Sigma^{-1 / 2} Q^{T} Q \Sigma^{-1 / 2}= \\
\Sigma^{-1 / 2} I \Sigma^{-1 / 2}=\Sigma^{-1}
\end{gathered}
$$

Since $Q$ was arbitrary, there are infinitely many choices of orthogonal matrices that will acomplish our task. Following and elaborating on the proof given by [KLS18], we would like to transform our sample vectors in such a way that we decorrelate their parameters by keeping the new vectors as close as possible to the original ones; in other words, we define an optimal whitening transformation if it removes the correlations but at the same time it minimizes the total squared distance between the original and whitened random variables.

After having centered the original random vector $x$ with mean $E[x]=0$, and let $z=W x$, since $W$ is a linear transformation, the mean of the random vector $z$ is $E[z]=E[W x]=$ $W(E[x])=W(0)=0$, thus $E[z]=0$.

As before, let

$$
E\left[x x^{T}\right]=\operatorname{cov}(x, x)=\operatorname{var}(x)=\Sigma,
$$

and by definition of $W$,

$$
E\left[z z^{T}\right]=\operatorname{cov}(z, z)=\operatorname{var}(z)=I,
$$

with

$$
E\left[z x^{T}\right]=\operatorname{cov}(z, x)=\operatorname{cov}(W x, x)=W \operatorname{cov}(x, x)=W \Sigma
$$


Our objective is to choose a whitening transformation $W$ that minimizes the total squared distance, i.e., that minimizes $(z-x)^{T}(z-x) \in \mathbb{R}_{+}$, between $z$ and $x$ :

$$
\begin{gathered}
E\left[(z-x)^{T}(z-x)\right]=E\left[z^{T} z-x^{T} z-z^{T} x+x^{T} x\right]= \\
E\left[\operatorname{tr}\left(z z^{T}\right)-\operatorname{tr}\left(z x^{T}\right)-\operatorname{tr}\left(x z^{T}\right)+\operatorname{tr}\left(x x^{T}\right)\right]= \\
E\left[\operatorname{tr}\left(z z^{T}\right)-\operatorname{tr}\left(z x^{T}\right)-\operatorname{tr}\left(\left(x z^{T}\right)^{T}\right)+\operatorname{tr}\left(x x^{T}\right)\right]= \\
E\left[\operatorname{tr}\left(z z^{T}\right)-2 \operatorname{tr}\left(z x^{T}\right)+\operatorname{tr}\left(x x^{T}\right)\right]= \\
\operatorname{tr}\left(E\left[z z^{T}\right]\right)-2 \operatorname{tr}\left(E\left[z x^{T}\right]\right)+\operatorname{tr}\left(E\left[x x^{T}\right]\right)= \\
\operatorname{tr}(\operatorname{cov}(z, z))-2 \operatorname{tr}(\operatorname{cov}(z, x))+\operatorname{tr}(\operatorname{cov}(x, x))= \\
\operatorname{tr}(I)-2 \operatorname{tr}(W \Sigma)+\operatorname{tr}(\Sigma)= \\
n-2 \operatorname{tr}\left(Q \Sigma^{1 / 2}\right)+\operatorname{tr}(\Sigma)
\end{gathered}
$$

In these derivations, we used the equality between the inner product $\mathbf{x}^{\mathbf{T}} \mathbf{x}$ and the trace $\operatorname{tr}\left(\mathbf{x x}^{\mathbf{T}}\right)$, properties of the trace, and the lineareity of and the commutativity between the Trace and Expected value operators. So, we need to minimize:

$$
E\left[(z-x)^{T}(z-x)\right]=n-2 \operatorname{tr}\left(Q \Sigma^{1 / 2}\right)+\operatorname{tr}(\Sigma)
$$

The covariance $\Sigma$ is symmetric; thus, let $\Sigma=U \Lambda U^{T}$ be its eigendecomposition yielding $\Sigma^{1 / 2}=U \Lambda^{1 / 2} U^{T}$, and $Q \Sigma^{1 / 2}=Q U \Lambda^{1 / 2} U^{T}$, with $Q, U$ and $U^{T}$ being orthogonal matrices, and diagonal $\Lambda^{1 / 2}$, which makes $\Lambda^{1 / 2}$ commute with any other matrix.

We can see that the problem of minimizing the squared distance in (3) is achieved by maximizing the trace of $Q \Sigma^{1 / 2}$; since $\operatorname{tr}(I)=n$ and $\operatorname{tr}(\Sigma)$ are independent of the choice of whitening transformation $W$. 
We show this maximization is equivalent to $Q=I$ :

$$
\begin{gathered}
\operatorname{tr}\left(Q \Sigma^{1 / 2}\right)=\operatorname{tr}\left(Q U \Lambda^{1 / 2} U^{T}\right)=\operatorname{tr}\left(\Lambda^{1 / 2} Q U U^{T}\right)= \\
\operatorname{tr}\left(\Lambda^{1 / 2} Q\right)=\sum_{i} \Lambda_{i i}^{1 / 2} Q_{i i}
\end{gathered}
$$

Hence,

$$
\operatorname{tr}\left(Q \Sigma^{1 / 2}\right)=\sum_{i} \Lambda_{i i}^{1 / 2} Q_{i i}
$$

Now, the fact that $Q$ is orthogonal, means that its columns are orthonormal vectors, thus its entries $Q_{i j} \leq 1$; in particular $Q_{i i} \leq 1$.

Therefore, $\sum_{i} \Lambda_{i i}^{1 / 2} Q_{i i}$ in (4) is maximized when $Q_{i i}=1$, which is equivalent to $Q=I$ for orthogonal $Q$, minimizing the total squared distance.

Hence, $W=Q \Sigma^{-1 / 2}=\Sigma^{-1 / 2}$ is the optimal whitening transformation. We will denote it as

$$
W=W^{Z C A}=\Sigma^{-1 / 2}
$$

The process to decorrelate these parameters is called whitening. We start by expressing the data set as a matrix, in our case is a 3 by 3399 matrix, where each column represents one tile or data point, and the 3 rows are made of the three previously mentioned parameters: Water Column Depth, Turbidity, and Dissolved Oxygen.

Let $X$ be our data matrix or map of size $d \times k$, where $d$ is the number of features and $k$ is the number observations. We approximate the covariance of the random vector $x$ by computing the sample covariance $X X^{T}$ of the data set $X$ up to a scalar as follows:

$$
\operatorname{cov}(y, y) \approx \frac{Y Y^{T}}{k-1}=: \Sigma
$$


The optimal whitening transformation for our data is given by

$$
W^{Z C A}=\Sigma^{-\frac{1}{2}}
$$

We apply the linear transformation in equation (2) to the whole data set, and to the new observation points to be localized. The distance between an observation and the whole map is taken and candidates are sorted in ascending order.

In our test, we only used the three parameters: Water Column Depth, Turbidity, and Dissolved Oxygen. We show the original (unwhitened) data in $\mathbb{R}^{3}$.

Table 6.1: Covariance matrix of data set with three parameters

\begin{tabular}{|c||c|c|c|}
\hline & Depth & ODO & Turbidity \\
\hline \hline Depth & 57.9896333 & -1.78032044 & 0.0704295744 \\
\hline ODO & -1.78032044 & 0.122827574 & -0.00970728820 \\
\hline Turbidity & 0.0704295744 & -0.00970728820 & 0.221983722 \\
\hline
\end{tabular}

If we plot the original data set, and from the covariance matrix above, we can see the parameters are somewhat correlated, as can be seen in Fig 6.3-a. A plot of the data after it has been dispersed (whitened) is shown in Fig 6.3-b.

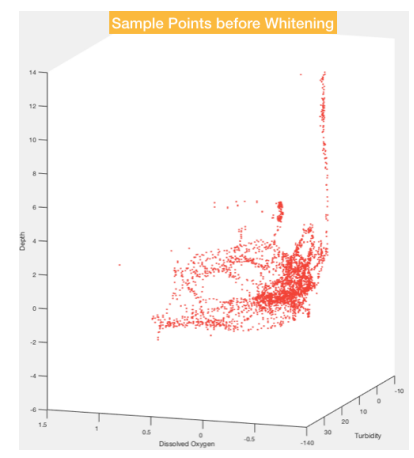

(a)

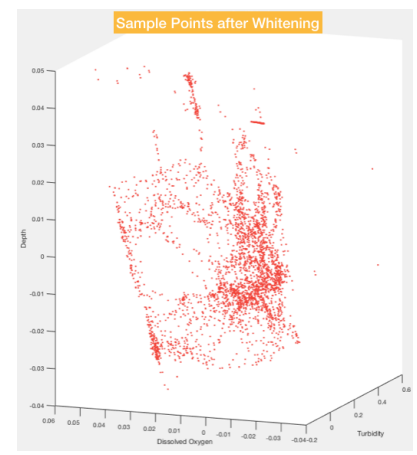

(b)

Figure 6.3: a) Original centered data and (b) Eigenvectors of the covariance matrix. 


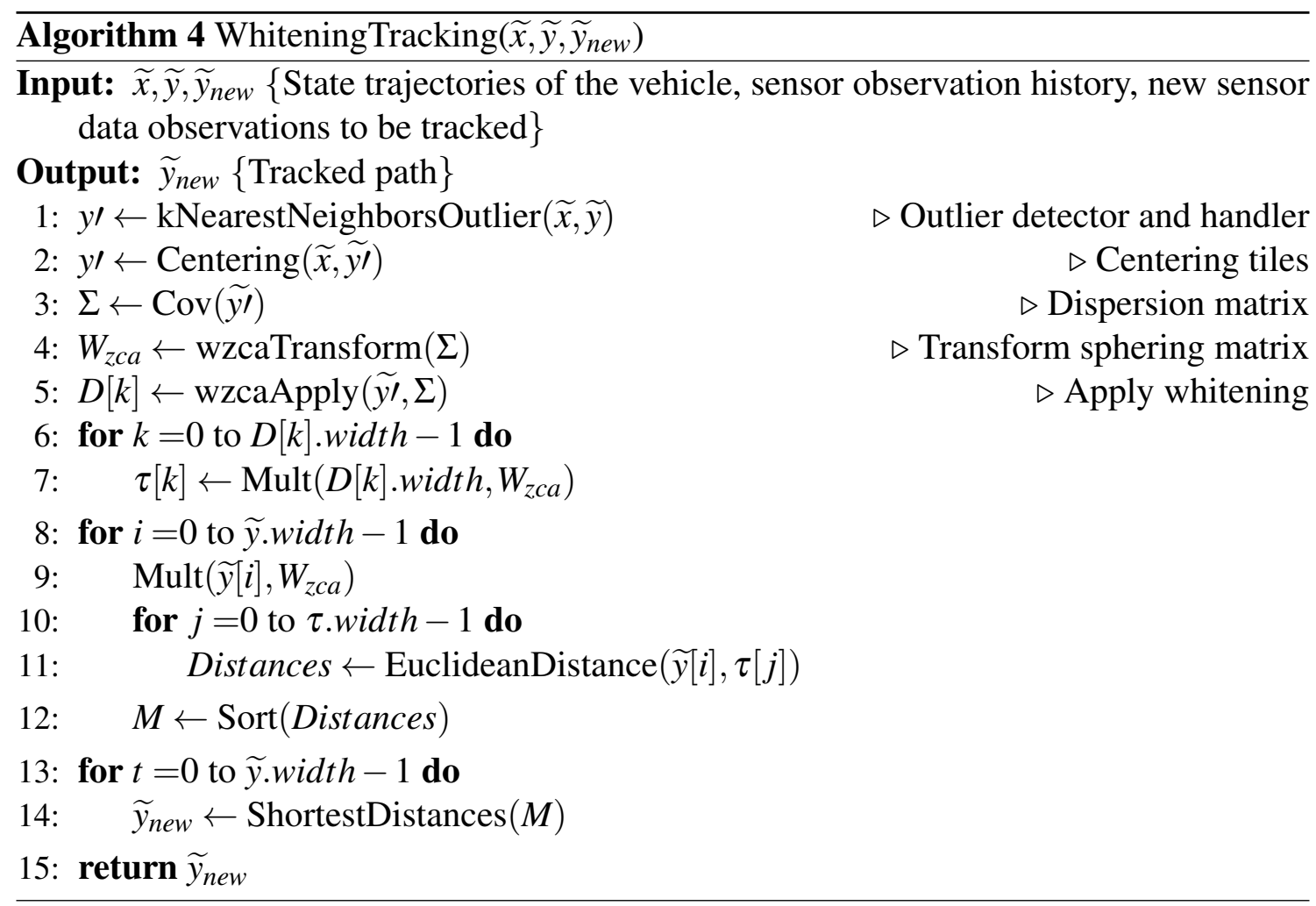

According to [KLS18], one important thing to highlight is that there are infinitely possible ways of creating whitening procedures that satisfy the constraint for the underlying whitening matrix 6.5. Therefore, since our goal is to obtain sphered variables which are maximally similar to the original ones, ZCA-cor was the whitening methodology chosen for this research. Still according to [KLS18], if maximal compression is the goal of the application, PCA-cor whitening becomes the best methodology.

\subsubsection{Localization Algorithm}

Once the GPS signal is lost, we perform the whitening on the map and the new observation point, and having sorted the candidates, we take the statistically closest one from our map to the new observation, within a radius equal to the average velocity times the elapsed time around the source or last GPS data point available. This average velocity is also measured 


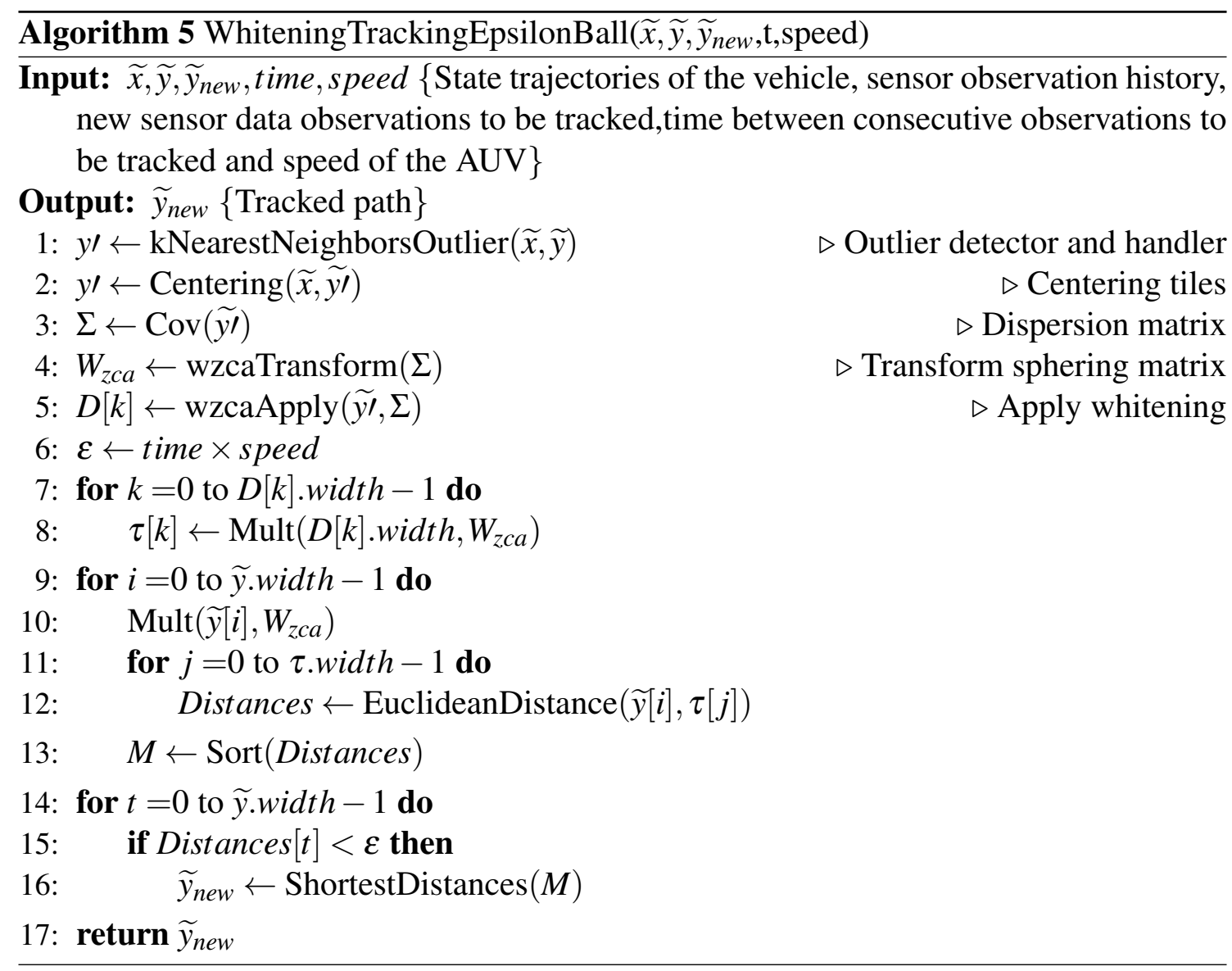

through non-GPS means (part of the AUV sensor data). If no candidate lie within that radius, we ignore that first observation point and after receiving the second one, we apply the same procedure from the source point with an augmented radius according to the elapsed time and average velocity. On the other hand, if the first observation was localized within the first radius, we mark that candidate as part of the new trajectory and draw a new radius around that point to localize the next observation point, and so on.

\subsubsection{Results in Santa Catalina Island, CA}

Our simulation results showed that this method works for a map that had been recently (within a couple of hours) recorded when compared to the time in which the observations to be localized were taken. The whitening of the data had positive results when compared 
to localizing without whitening. The first test was performed on the dataset collected on the first day of deployment. Fig. 6.4 shows the performed trajectory of the robot during deployment (in blue) and the green points represent a list of observations where GPS signal was unavailable and the orange ones represent the approximate localization of these observations without whitening the data and including the whitening. Fig. 6.6 illustrate the same approach on the second day of deployment.

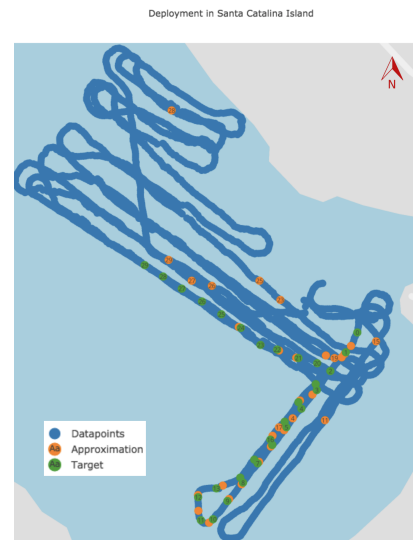

(a)

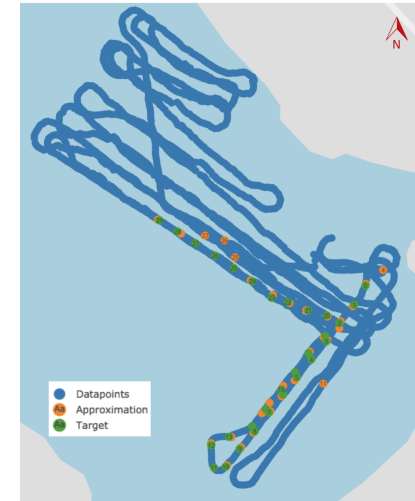

(b)

Figure 6.4: Blue points represent the data-points during the first day of deployment; the green points represent the list of observations where GPS signal was not available; and the orange points represent the approximate localization of each one of these observations a) without the whitening process and $b$ ) using the whitening process. 


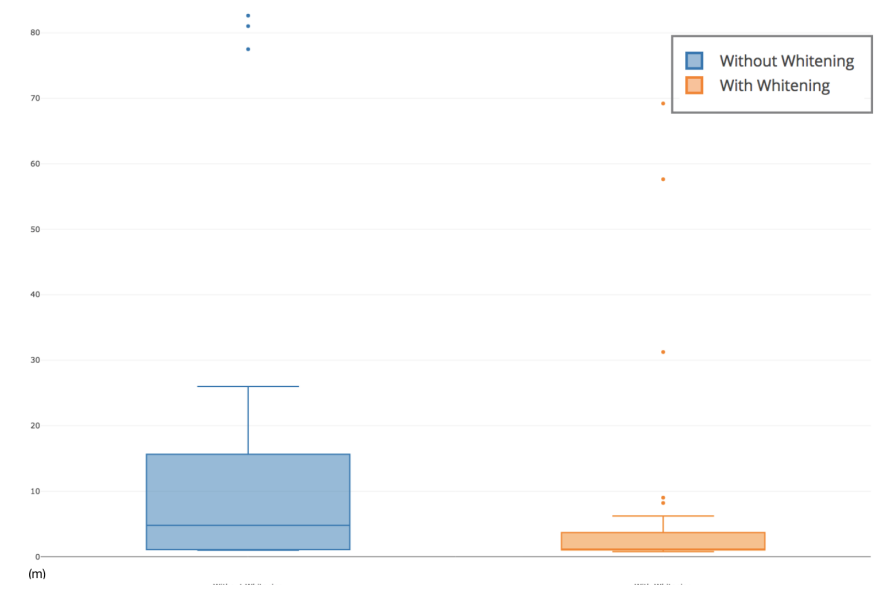

Figure 6.5: Comparison of the errors in GPS information using without whitening and with whitening methods for the first day of deployment.

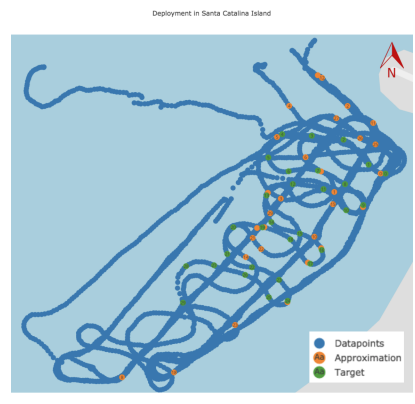

(a)

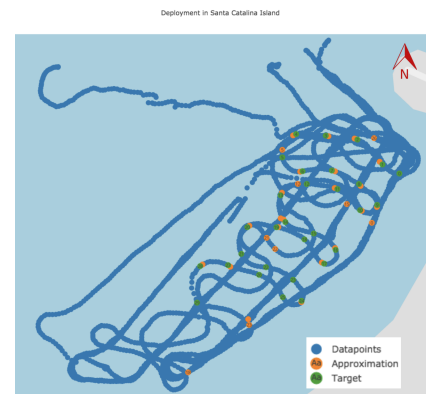

(b)

Figure 6.6: Blue points represent the data-points during the second day of deployment; the green points represent the list of observations where GPS signal was not available; and the orange points represent the approximate localization of each one of these observations a) without the whitening process and $b$ ) using the whitening process. 


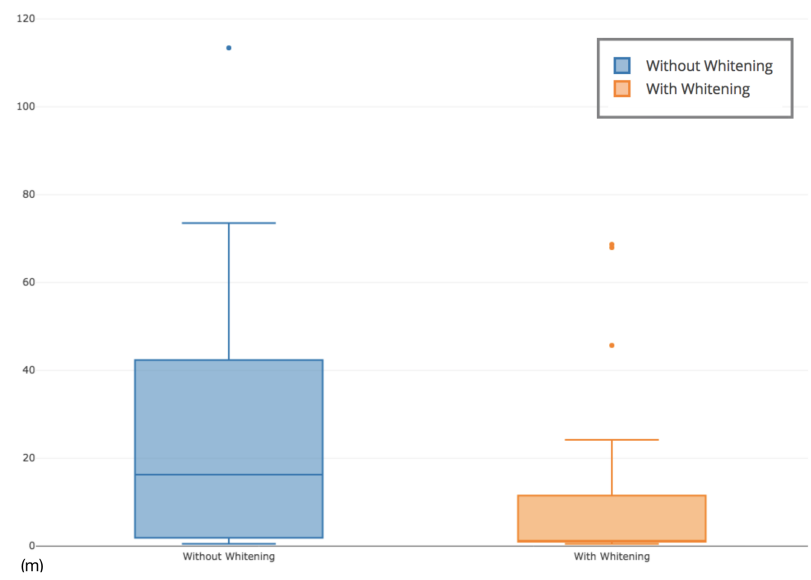

Figure 6.7: Comparison of the errors in GPS information using without whitening and with whitening methods for the second day of deployment.

On the first day of deployment, for the case where whitening was not used in the localization, median $M=4.78503$, first quartile $q_{1}=1.091315$, third quartile $q_{3}=15.6415$, minimum $\min =0.994319$, upperfence $=25.98387$, and maximum $\max =82.63074$, in meters $(\mathrm{m})$. By using the whitening process, $M=1.16423, q_{1}=1.038154, q_{3}=$ 3.67947, $\min =0.762205$, upperfence $=6.223385$, and $\max =69.21059$, in meters (m). The comparison of the errors in approximation using GPS information as ground truth can be seen in Fig. 6.8. On the second day of deployment, median $M=16.26253$, $q_{1}=1.887719, q_{3}=42.37872, \min =0.5108102$, upperfence $=73.53935$, and $\max =$ 113.417, in meters (m), when localization was attempted for the correlated data. By decorrelating the data, median $M=1.214548, q_{1}=0.977641, q_{3}=11.53782, \min =$ 0.5108102 , upperfence $=24.21372$, and $\max =68.68564$, in meters $(\mathrm{m})$. Differences between the days can be explained by the difference in the size of the dataset and also the period of the day when data was collected. 


\subsubsection{Results at the Lake Nighthorse, CO}

We collected fourteen datasets during eight missions executed during April 25, April 6, April 27, April 30, May 1, May 2, May 3, May 4, 2018. For most of them, we collected data in the morning and in the afternoon. One of the most interesting aspects of these missions is the occurrence of a plume in the morning of April 30, which changed some of the water parameters and allowed a deeper visualization of how the algorithm works. Furthermore, it snowed on May 2 at night, which caused a difference in some of the water parameters collected on May 3 in the morning.

Results showed that the temperature $\left({ }^{\circ} \mathrm{C}\right)$ of the water is the parameter that varies the most, together with turbidity (NTU) and dissolved oxygen $(\mathrm{mg} / \mathrm{L})$ are the most significant parameters, i.e. the principal components, used to enhance the depth (total water column) (m). Here, the localization algorithm begins once the GPS signal becomes unavailable and we wish to continue tracking the vehicle's state given the collected observations from the sensors. We do not use kinematics or sensors other than the water data sensors, the compass and the speed of the vehicle in order to track it.

Different missions were executed and are illustrated through boxplots and maps shown in Figs. 6.9-6.26. We compare the results in localization with the application of the whitening process and without it to motivate the novelty of this application. Missions varied according to the bathymetric relief (little to significant bathymetric relief), to the period of the day (morning and afternoon), same day or different days (1 day apart and 1 week apart)) and were also executed using the concept of epsilon ball and without the use of epsilon ball motivating the use of the speed of the vehicle as a filter for better localization results. 


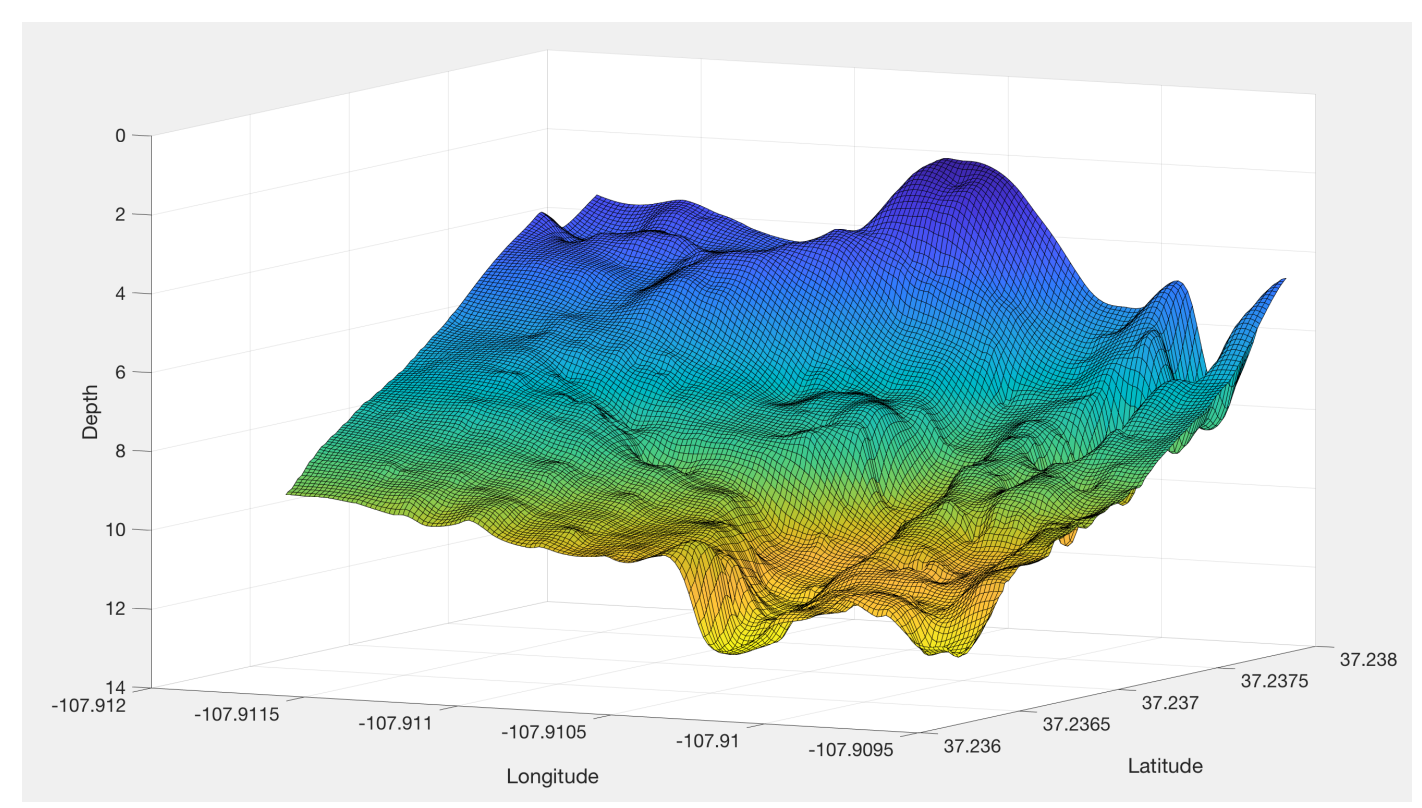

Figure 6.8: The bathymetric map of the region of interest at the Lake Nighthorse, CO.

\section{MISSION 1 - same day with significant relief}

This test was done by using a trajectory extracted from a mission on April 25th 2018 and localizing this trajectory on the reference map created on the same day. The trajectory was chosen due to its significant relief. Algorithm NB was applied to be later compared with Algorithm EB. 


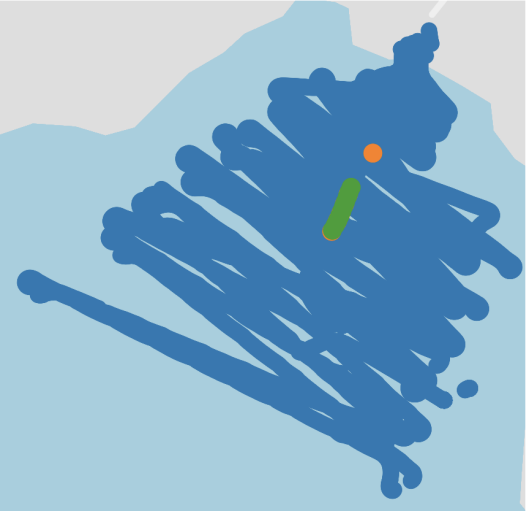

(a)
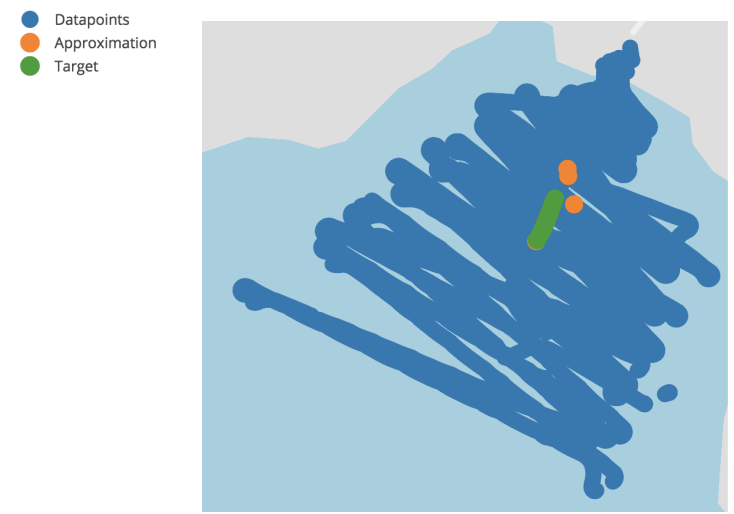

(b)

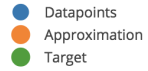

Figure 6.9: The localized observations are shown in orange, while the original trajectory is shown in green. This trajectory was selected for test due to significant bathymetric relief. Blue points represent surveyed data for the first mission executed on April 25th 2018. In (a) the observations were localized on without the whitening process and in (b) the whitening process was applied

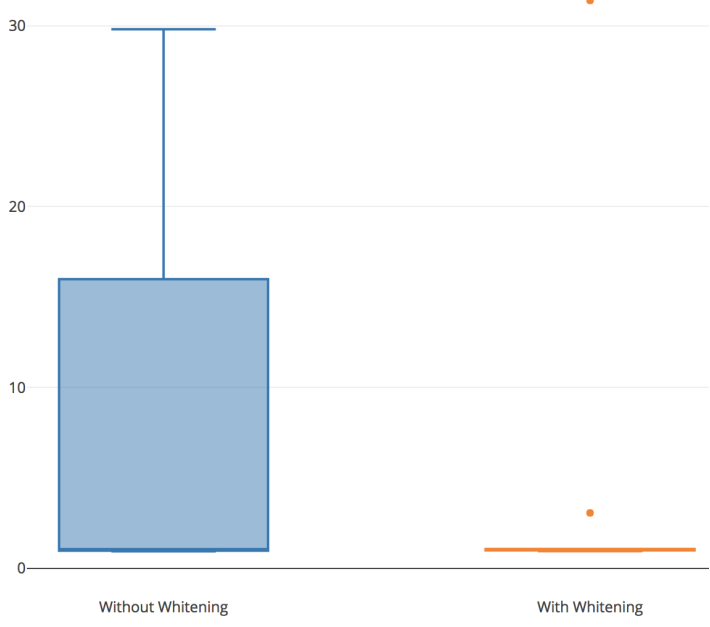

Figure 6.10: Comparison of the errors in GPS information without the whitening process (in blue) and with the whitening process (in orange) for the first mission executed on April 252018

\section{MISSION 2 - same day with little relief}

This test was done by using a trajectory extracted from a mission on April 252018 and localizing this trajectory on the reference map created on the same day. The trajectory 
was chosen due to its little relief. Algorithm NB was applied to be later compared with Algorithm EB.

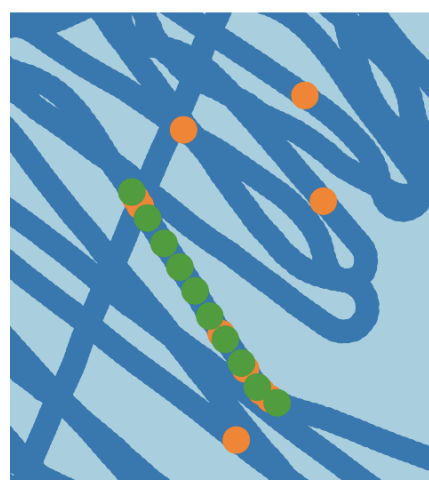

(a)

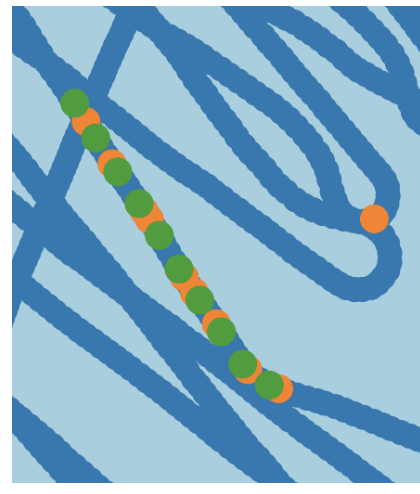

(b)

Figure 6.11: The localized observations are shown in orange, while the original trajectory is shown in green. This trajectory was selected for test due to significant bathymetric relief. Blue points represent surveyed data for the first mission executed on April 25th 2018. In (a) the observations were localized on without the whitening process and in (b) the whitening process was applied.

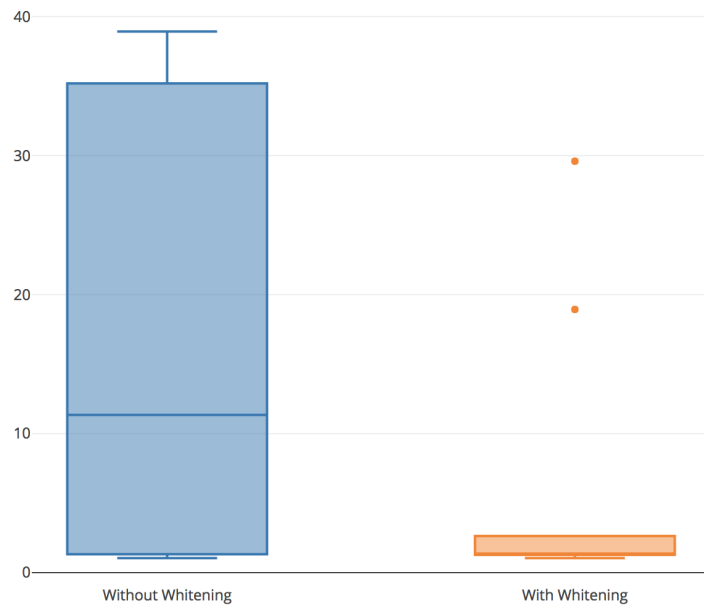

Figure 6.12: Comparison of the errors in GPS information without the whitening process (in blue) and with the whitening process (in orange) for the first mission executed on April 252018.

Results for missions executed on the same day when the reference map was created showed improvement in the average error in localization of up to $10 \mathrm{~m}$. It is important to 
highlight that the bathymetric relief played a minor role since the other parameters of the water provided great variance and contributed more to the improvement in accuracy.

\section{MISSION 3 - same day with little relief and epsilon ball}

This test was done by using a trajectory extracted from a mission on April 252018 and localizing this trajectory on the reference map created on the same day. The trajectory was chosen due to its little relief. Algorithm EB was applied due to little information on the depth parameter.

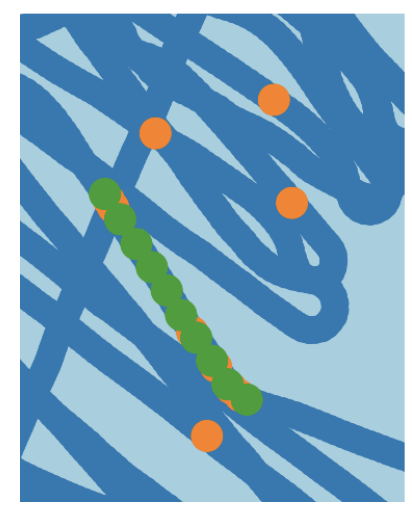

(a)
Datapoints Approximation Target

Figure 6.13: The localized observations are shown in orange, while the original trajectory is shown in green. This trajectory was selected for test due to significant bathymetric relief. Blue points represent surveyed data for the first mission executed on April 25th 2018. In (a) the observations were localized on without the whitening process and in (b) the whitening process was applied with epsilon ball.

Results here showed that the epsilon ball was able to handle outliers and dramatically decreased the maximum value of the average errors by capturing points that were not correctly localized by the previous algorithm.

\section{MISSION 4 - 1 week apart with significant relief and epsilon ball}

This test was done by using a trajectory extracted from a mission on May 2nd 2018 and localizing this trajectory on the reference map created on April 25th 2018. The trajectory 


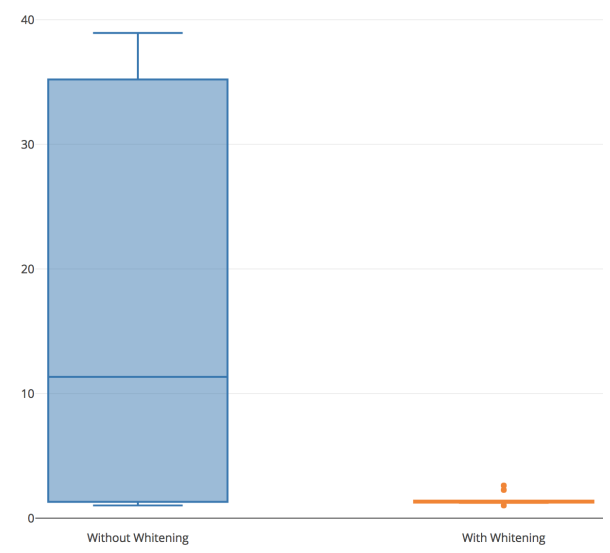

Figure 6.14: Comparison of the errors in GPS information without the whitening process (in blue) and with the whitening process (in orange) for the first mission executed on April 25th 2018.

was chosen due to its significant relief. Algorithm EB was applied because even though there was significant information on the depth parameter, the other variables presented changes within a week.

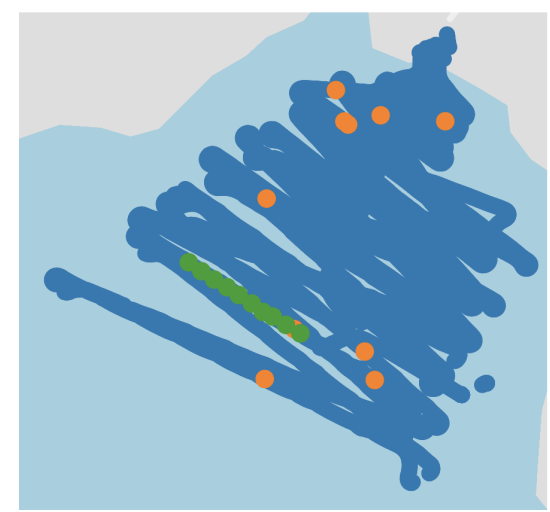

(a)
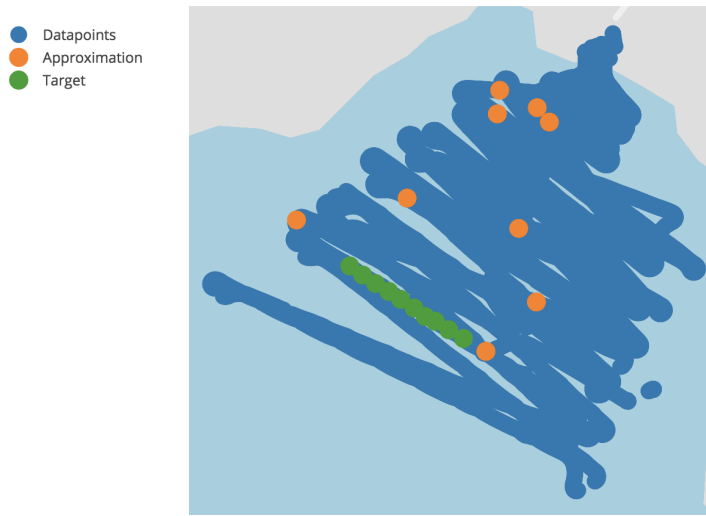

(b)

Figure 6.15: The localized observations are shown in orange, while the original trajectory (extracted from a mission on May 2nd) is shown in green. This trajectory was selected for test due to significant bathymetric relief. Blue points represent surveyed data for the first mission executed on April 25th 2018. In (a) the observations were localized on without the whitening process and in (b) the whitening process was applied with epsilon ball. 

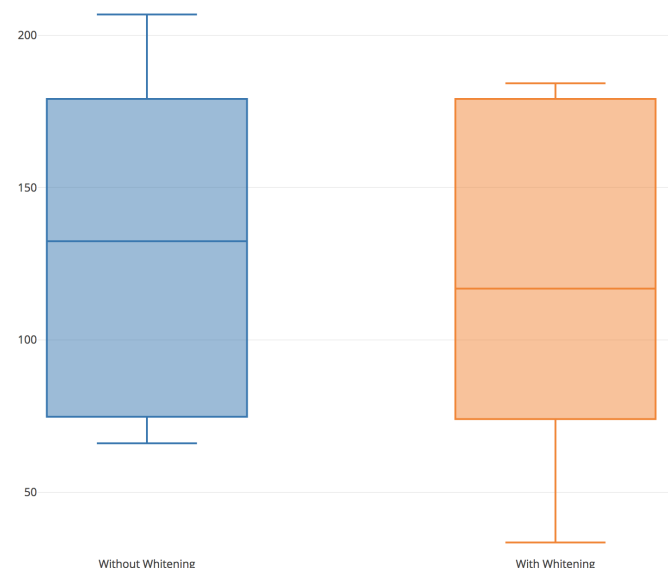

Figure 6.16: Comparison of the errors in GPS information without the whitening process (in blue) and with the whitening process (in orange).

Results for missions executed in one week apart from when the reference map was created showed improvement in the average error in localization of up to $16 \mathrm{~m}$. It is important to highlight that the even though there was a improvement, the average error in localization was $11.83 \mathrm{~m}$.

\section{MISSION 5 - 1 week apart with little relief and close to the shore}

This test was done by using a trajectory extracted from a mission on May 2nd 2018 and localizing this trajectory on the reference map created on April 25th 2018. The trajectory was chosen not only due to its little relief but also for being close to the shore. We used algorithm NB. 


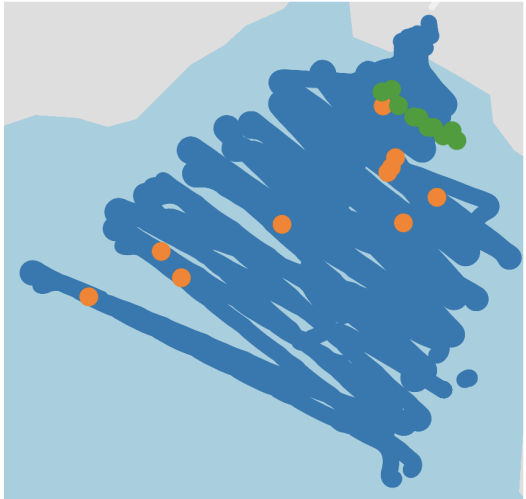

(a)

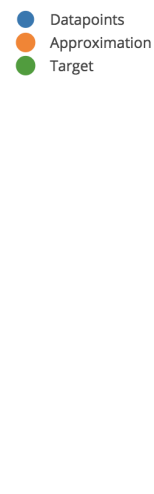

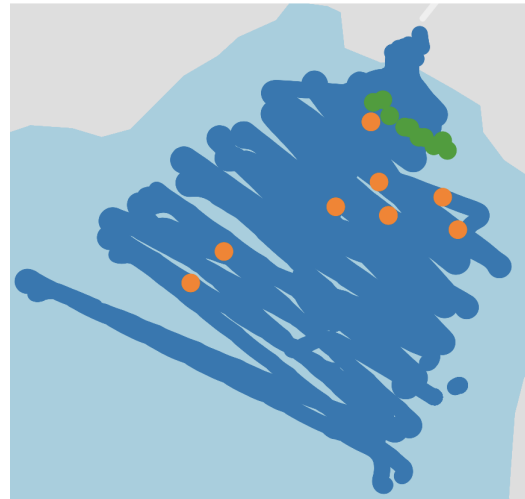

(b)

Figure 6.17: The localized observations are shown in orange, while the original trajectory (extracted from a mission on May 2nd) is shown in green. This trajectory was selected for being close to the shore. Blue points represent surveyed data for the first mission executed on April 25th 2018. In (a) the observations were localized on without the whitening process and in (b) the whitening process was applied.

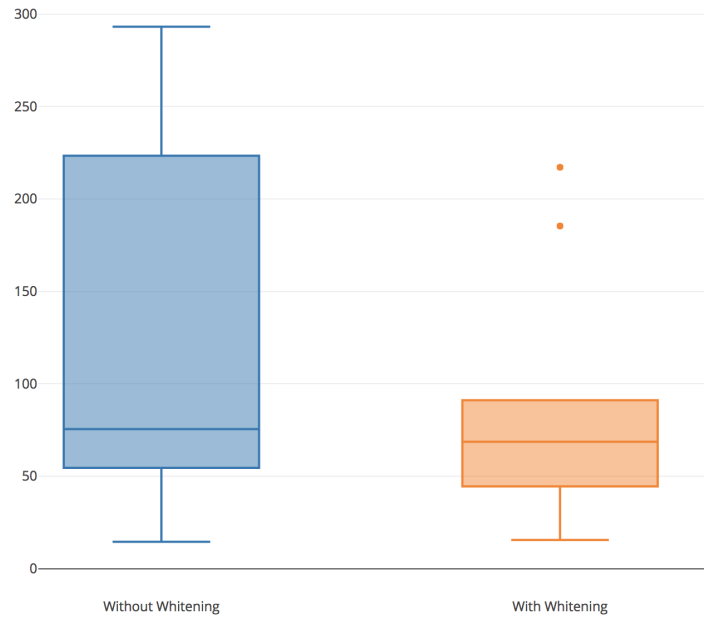

Figure 6.18: Comparison of the errors in GPS information without the whitening process (in blue) and with the whitening process (in orange).

Results for this missions was important to motivate the use of the epsilon ball (using the speed of the vehicle) to capture outliers and points that were localized far from the location of the deployment. 


\section{MISSION 6 - same day with significant relief}

This test was done by using a trajectory extracted from a mission on May 2nd 2018 and localizing this trajectory on the reference map created on the same day. The trajectory was chosen due to its little relief and proximity to the shore. Algorithm NB was applied to be later compared with Algorithm EB in mission 7.

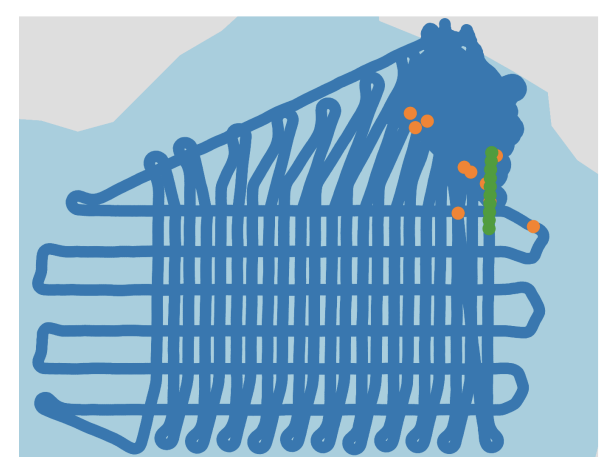

(a)
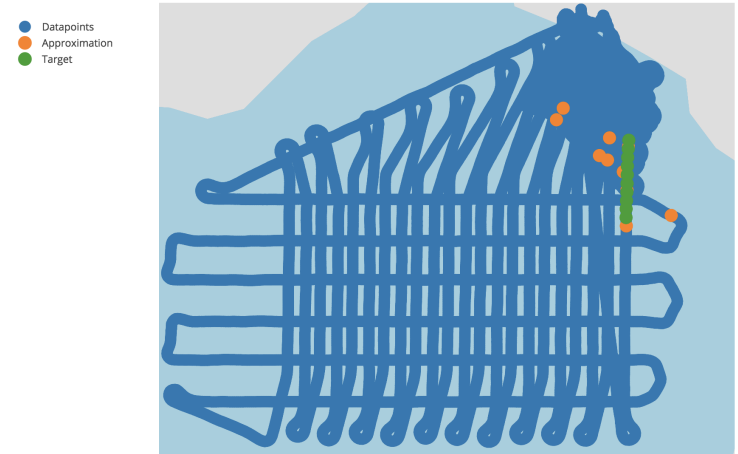

(b)

Figure 6.19: The localized observations are shown in orange, while the original trajectory (extracted on the same day) is shown in green. This trajectory was selected for being close to the shore. Blue points represent surveyed data for the mission executed on May 2nd 2018. In (a) the observations were localized on without the whitening process and in (b) the whitening process was applied.

Even though this mission was executed on the same day when the reference map was created (morning), the importance lies in the fact that during the night before the deployment was executed. Here we should analyze two aspects: (i) the application of whitening to the data generated an improvement in the average error in localization of up to $7 \mathrm{~m}$ compared to not using the whitening technique; and (ii) the fact that it rained during the night before the deployment increased the average error in localization, $11 \mathrm{~m}$ greater the average error calculated for the mission when there was no rain the night before. 


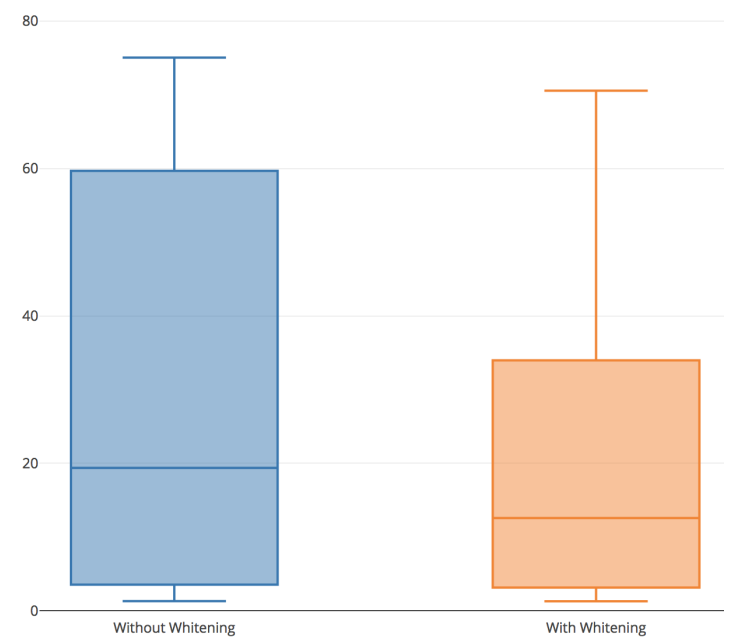

Figure 6.20: Comparison of the errors in GPS information without the whitening process (in blue) and with the whitening process (in orange).

\section{MISSION 7 - same day with significant relief and epsilon ball}

This test was done by using a trajectory extracted from a mission on May 2nd 2018 and localizing this trajectory on the reference map created on the same day. The trajectory was chosen due to its little relief and proximity to the shore. Algorithm EB was applied to be later compared with Algorithm NB in mission 6.

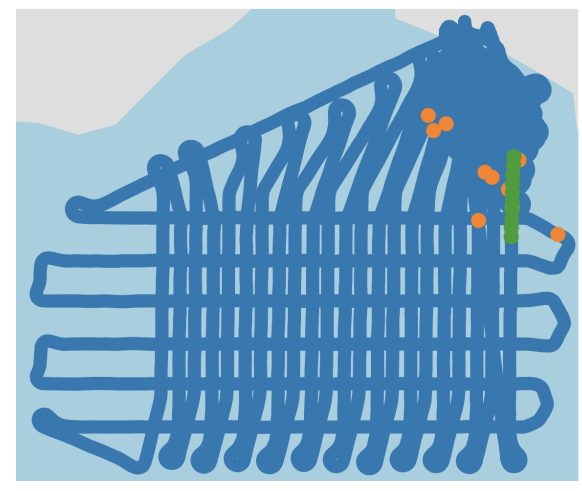

(a)
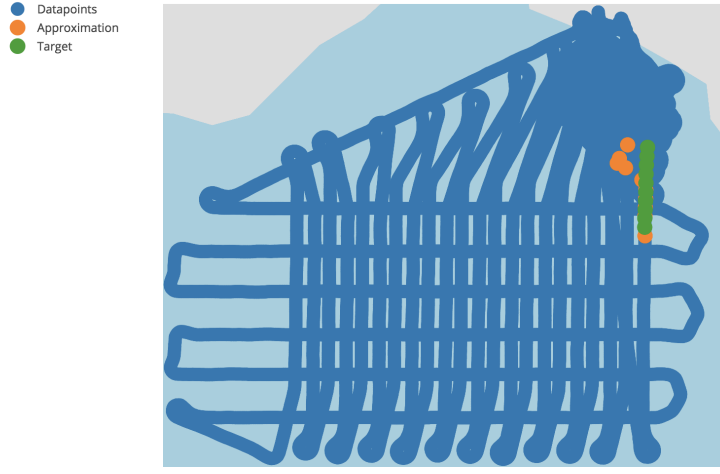

(b)

Figure 6.21: The localized observations are shown in orange, while the original trajectory (extracted on the same day) is shown in green. This trajectory was selected for being close to the shore. Blue points represent surveyed data for the mission executed on May 2nd 2018. In (a) the observations were localized on without the whitening process and in (b) the whitening process was applied with epsilon ball. 


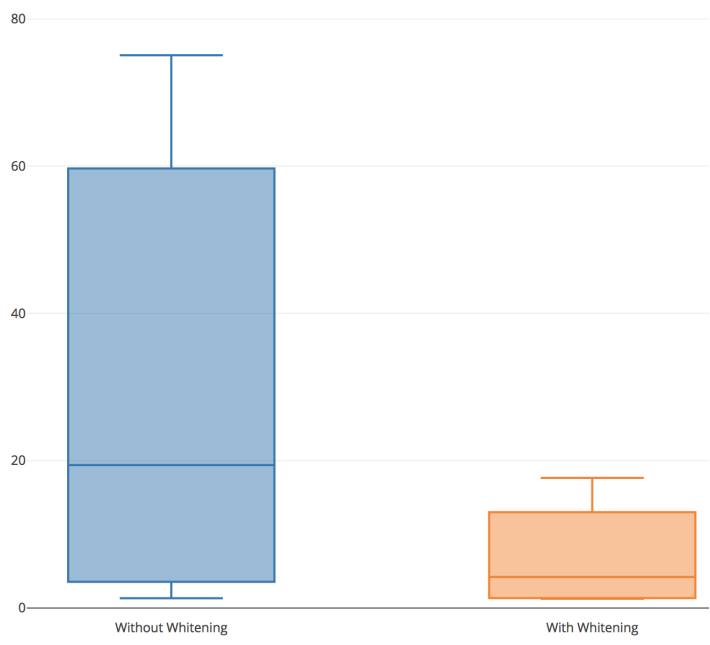

Figure 6.22: Comparison of the errors in GPS information without the whitening process (in blue) and with the whitening process (in orange).

This mission shows the improvement in the average error in localization when we use the epsilon ball algorithm. The average error in localization when the epsilon ball algorithm was used is $4.19 \mathrm{~m}$. When compared to the mission above (Mission 6), average error of localized was decreased by $8 \mathrm{~m}$.

\section{MISSION 8 - same day (morning and afternoon) with significant relief}

This test was done by using a trajectory extracted from a mission on May 2nd 2018 at late afternoon and localizing this trajectory on the reference map created on the same day, but early morning. The trajectory was chosen due to its significance relief and proximity to the shore. Algorithm NB was applied to be later compared with Algorithm EB in mission 9. 


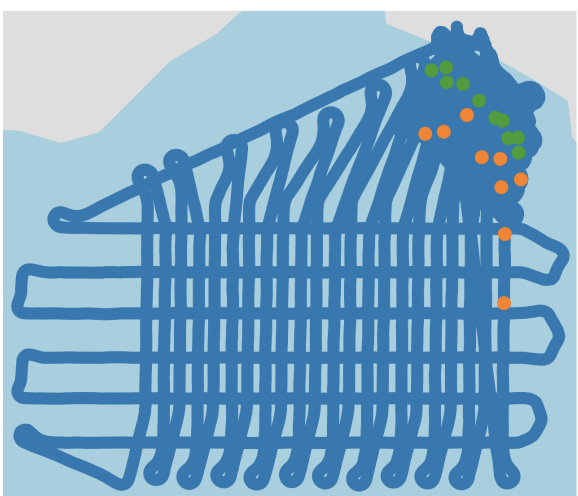

(a)
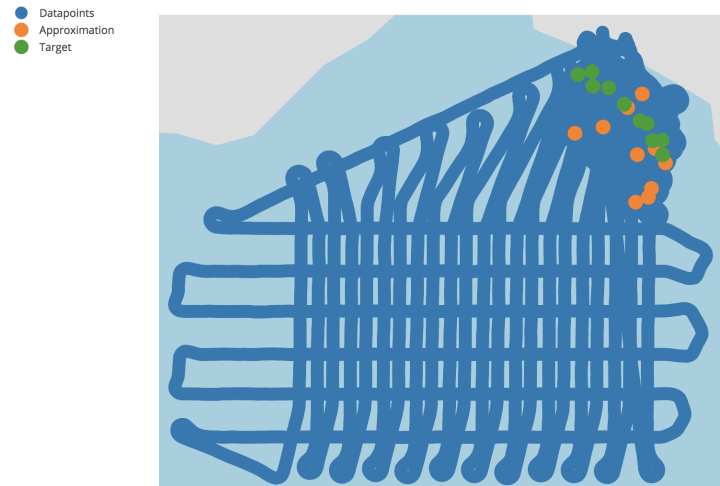

(b)

Figure 6.23: The localized observations are shown in orange, while the original trajectory (extracted on the same day, but in the afternoon) is shown in green. This trajectory was selected for being close to the shore and with significant relief. Blue points represent surveyed data for the mission executed on May 2nd 2018 in the morning. In (a) the observations were localized on without the whitening process and in (b) the whitening process was applied with epsilon ball.

The results for this case showed an increase in the average error in localization due to the fact that another mission was executed on the same day of missions 6 and 7, but in the later afternoon. The reference map was generated in the morning of the same, after the rain. Here, we can analyze the due to the rain, a reference map created in the morning and a mission executed in the afternoon had an average error in localization of $38.51 \mathrm{~m}$. This is still more accurate than the same test but without the application of the whitening process, whose average error in localization was $48.71 \mathrm{~m}$. 

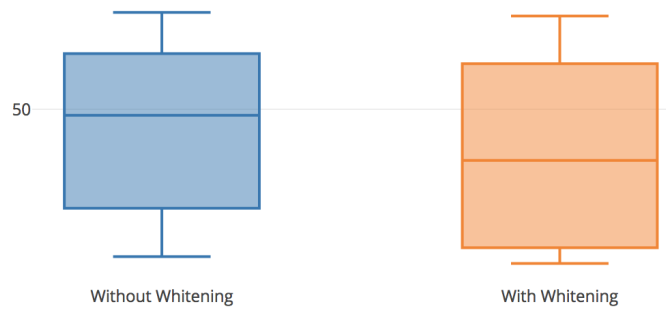

Figure 6.24: Comparison of the errors in GPS information without the whitening process (in blue) and with the whitening process (in orange).

MISSION 9 - same day (morning and afternoon) with significant relief and epsilon ball

This test was done by using a trajectory extracted from a mission on May 22018 at late afternoon and localizing this trajectory on the reference map created on the same day, but early morning. The trajectory was chosen due to its significance relief and proximity to the shore. Algorithm EB was applied to be later compared with Algorithm NB in mission 8. 


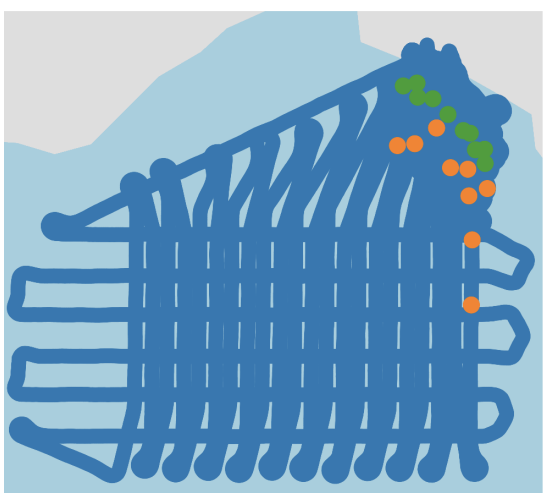

(a)
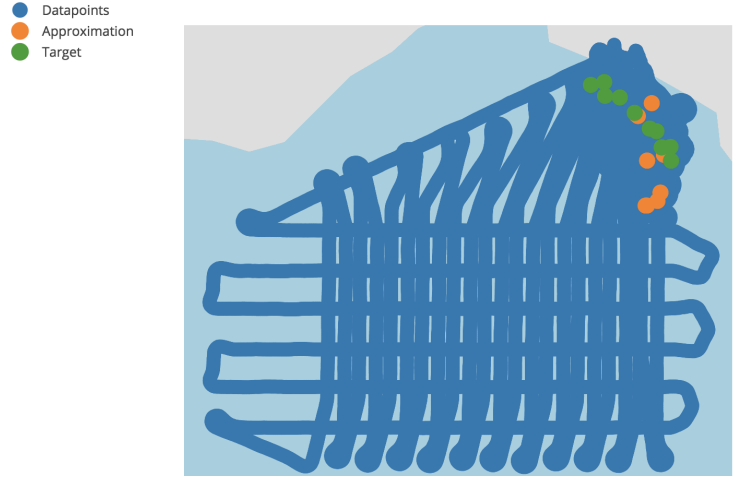

(b)

Figure 6.25: The localized observations are shown in orange, while the original trajectory (extracted on the same day, but in the afternoon) is shown in green. This trajectory was selected for being close to the shore and with significant relief. Blue points represent surveyed data for the mission executed on May 22018 in the morning. In (a) the observations were localized on without the whitening process and in (b) the whitening process was applied with epsilon ball.

The result of this mission showed an improvement of $4 \mathrm{~m}$ in the average error in localization when the epsilon ball was used to capture outliers and points localized far from the deployment location.

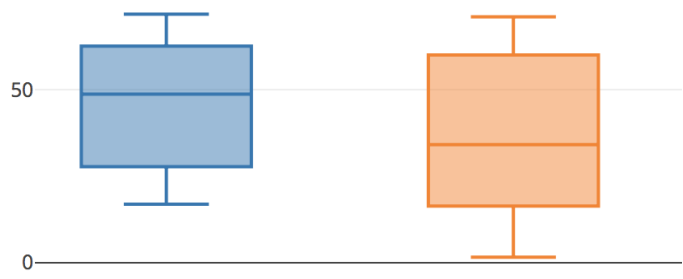

Without Whitening

With Whitening

Figure 6.26: Comparison of the errors in GPS information without the whitening process (in blue) and with the whitening process (in orange).

The numerical results for the previous boxplots and the information on the first quartile (Q1), minimum, maximum and the third quartile (Q3) values can be seen in Table 6.2. 
The values are in meters (m) and compare the results from different missions using the proposed whitening approach and without using this approach.

\subsubsection{Conclusion and Future Work}

Whitening is a promising technique for localization of underwater vehicles in GPS-denied environments using water science parameters. Assuming the data map is recent with respect to the newly observed data points, this method provides a reliable way of tracking and localizing the vehicle with acceptable precision (when compared to GPS). One limitation of this method is that the time of the day when the observations to be localized are taken must be similar to the time when the sampled observations were obtained. Moreover, an optimal path for data collection and further analysis of the temporal aspects of the properties of the water parameters are necessary for the applicability of the methodology in a larger area of interest. We tested different scenarios, where there is little or significant bathymetric relief, compared trajectories from one day and the map created with data from the same day, one week apart or different parts of the day. Also, comparison using different algorithms developed in this chapter were presented. These results show us that these additional water parameters can only improve the previous terrain maps obtained by only using water depth columns and how the developed algorithms can aid in navigation and tracking of underwater vehicles. 
Table 6.2: Interpretation of the boxplots for multiple missions

\begin{tabular}{|c|c|c|c|c|c|c|}
\hline & $\begin{array}{l}\text { Whitening } \\
\text { Method }\end{array}$ & $\operatorname{Max}$ & Q3 & Median & Q1 & Min \\
\hline \multirow{2}{*}{$\begin{array}{l}\text { Mission } 1 \text { - Significant } \\
\text { bathymetric relief and } \\
\text { same day }\end{array}$} & Without & 29.80 & 15.98 & 1.06 & 0.97 & 0.94 \\
\hline & With & 31.39 & 1.07 & 1.04 & 0.99 & 0.96 \\
\hline \multirow{2}{*}{$\begin{array}{l}\text { Mission } 2 \text { - Little } \\
\text { bathymetric relief and } \\
\text { same day }\end{array}$} & Without & 38.94 & 35.21 & 11.35 & 1.32 & 1.03 \\
\hline & With & 29.60 & 2.63 & 1.35 & 1.27 & 1.03 \\
\hline \multirow{2}{*}{$\begin{array}{l}\text { Mission } 3 \text {-Little } \\
\text { bathymetric relief, } \\
\text { same day and } \\
\text { epsilon ball }\end{array}$} & Without & 38.94 & 35.21 & 11.35 & 1.32 & 1.03 \\
\hline & With & 2.63 & 1.41 & 1.32 & 1.26 & 1.03 \\
\hline \multirow{2}{*}{$\begin{array}{l}\text { Mission } 4 \text { - Significant } \\
\text { bathymetric relief with } \\
\text { epsilon ball and one } \\
\text { week apart }\end{array}$} & Without & 206.80 & 179.10 & 132.39 & 74.78 & 66.06 \\
\hline & With & 191.33 & 181.24 & 116.83 & 74.01 & 33.53 \\
\hline \multirow{2}{*}{$\begin{array}{l}\text { Mission } 5 \text { - Little } \\
\text { bathymetric relief and } \\
\text { one week apart }\end{array}$} & Without & 293.19 & 223.40 & 75.48 & 54.49 & 14.54 \\
\hline & With & 217.13 & 91.10 & 68.64 & 44.50 & 15.49 \\
\hline \multirow{2}{*}{$\begin{array}{l}\text { Mission } 6 \text { - Significant } \\
\text { bathymetric relief and } \\
\text { same day }\end{array}$} & Without & 75.06 & 59.69 & 19.38 & 3.53 & 1.31 \\
\hline & With & 70.57 & 33.98 & 12.57 & 3.15 & 1.28 \\
\hline \multirow{2}{*}{$\begin{array}{l}\text { Mission } 7 \text { - Significant } \\
\text { bathymetric relief with } \\
\text { epsilon ball and same } \\
\text { day }\end{array}$} & Without & 75.06 & 59.69 & 19.38 & 3.53 & 1.31 \\
\hline & With & 17.65 & 12.99 & 4.19 & 1.32 & 1.24 \\
\hline \multirow{2}{*}{$\begin{array}{l}\text { Mission } 8 \text { - Significant } \\
\text { bathymetric relief and } \\
\text { morning/afternoon }\end{array}$} & Without & 131.28 & 62.62 & 48.71 & 27.71 & 16.83 \\
\hline & With & 71.08 & 60.32 & 38.51 & 18.81 & 15.29 \\
\hline \multirow{2}{*}{$\begin{array}{l}\text { Mission } 9 \text { - Significant } \\
\text { bathymetric relief with } \\
\text { ball and morning/afternoon }\end{array}$} & Without & 131.28 & 62.62 & 48.71 & 27.71 & 16.83 \\
\hline & With & 71.08 & 60.07 & 34.12 & 16.29 & 1.53 \\
\hline
\end{tabular}




\section{CHAPTER 7}

\section{CONCLUSION}

Effective observation and quantification of spatio-temporally dynamic processes occurring in aquatic environments, e.g., the ocean, requires simultaneous measurement of diverse water properties, which must be made rapidly to capture the both the spatial and temporal variability of multiple simultaneous interactions. Traditional oceanographic methods which involves infrequent and sparse measurements from ships, buoys and drifters are not always able to capture these dynamics. Moreover, underwater vehicles commonly perform underwater navigation using dead-reckoning approaches using accelerometers, magnetometers and depth sensors. Nonetheless, these instruments are subject to large drift which, in turn, leads to unbounded uncertainty in location. Coupled with the dynamic environment of the ocean, the state estimate of location can vary significantly from the actual location. In order to overcome these location estimate problems, the AUVs are sent to the surface for a GPS fix, which can cause risks to the vehicles due to obstacles e.g., ships, boats and rocks or researchers integrate more accurate sensors which not only increases the cost of the experiment but also requires more energy. Both solutions also decrease the time that the AUV could use for sampling, reducing the deployment duration.

Even with higher resolution bathymetric maps, traditional terrain-based navigation solutions can result in significant navigational error, especially in regions of little to no vertical relief. To improve the capability of the AUV to navigate and localize, we we examined an interpolation methodology, a kriging-based solution, for the problem of finding the value of the unknown locations in the environment. By computing the semi-variance of each water parameter and bathymetry and by measuring the spatial dependence between any two observations as a function of the distance between them, maps were created for each one of the water parameter and bathymetry. Based on the results from this pre- 
processing of the data, a data-driven approach for informative sampling of autonomous underwater vehicles was examined and presented. Using Markov Decision Process, an optimal navigation policy was presented in the 2-D marine environment that generates the best possible action in the simulated policy from any location of the environment to the goal location. This policy can be used to decrease the error in prediction in regions where a second mission is necessary. Thus, we were able to optimize time spent collecting data and reducing the errors in prediction for the sensor data.

In order to enhance the bathymetric maps used in regular terrain-based navigation approaches, an augmented TBN that incorporates physical science data, i.e., water parameters such as temperature, salinity, $\mathrm{pH}$, etc., to enrich the uniqueness of the underlying terrain map. We described in the details the process of creating such a combination map, starting from investigating the spatial autocorrelation of each water parameter and bathymetry, exploring different techniques for assigning weights to each of the parameters and finally combining the parameters in one unique reference map. It is also important to highlight is that such methodology of creating maps for localization and navigation can be extended from underwater environments to ground and aerial vehicles given the spatio-temporal dynamics of environment properties. One of the most exciting features of these reference maps is that they can be developed as the vehicles move and they are independent of the kinematics of the vehicles and they do not require a larger infrastructure and high intensive sensor like acoustic solutions.

Following the creation of the maps, a novel localization algorithm and two novel tracking algorithms were developed and tested with real data from multiple deployments in Big Fisherman's Cove in Santa Catalina Island, CA, USA and Lake Nighthorse, CO, USA between 2016 and 2018. Different missions were executed and localization was tested on the same day, different days and different weathers with and without snow. Moreover, a novel application for whitening methodologies was analyzed and explored in 
the tracking algorithms, where better precisions in localization were achieved when water data and bathymetry were whitened before being used for tracking.

These map generated with the combination of science parameters and bathymetry information can facilitate localization and navigation algorithms for underwater and surface vehicles with a vanilla application of traditional TBN methods. For most ocean science applications, there is a need for underwater vehicles to navigate within a spatio-temporally dynamic environments and to gather data of high scientific value. Here, instead of thinking of locations existing in geographic space, we consider them to be drawn from or existing in an environmental space. Coupled with physical models (predictive ocean models), this relaxes the dependence on geographic coordinates for navigation, and enables the deign of methods for improving navigation and sampling within a dynamic feature. The inclusion of depth as a parameter does serve to ground-truth this methodology as we continue to develop the supporting architecture for spatio-temporal dynamics.

Therefore, we developed approaches underpinning intelligent localization and navigation within oceanic features and coherent structures by AUVs. We based our strategies using current state-of-the-art ocean modeling, forecasting of biological phenomena, autonomous vehicle control localization and tracking, adaptive sampling techniques, scalar field reconstruction and environmental sensing. The scientific advances in this project will enable more effective navigation in the ocean monitoring, coral reefs preservation and the characterization of the biology and biochemical processes associated with spatiotemporally dynamic features. 


\section{BIBLIOGRAPHY}

[ABS18] Tauhidul Alam, Leonardo Bobadilla, and Dylan A Shell. Space-efficient filters for mobile robot localization from discrete limit cycles. IEEE Robotics and Automation Letters, 3(1):257-264, 2018.

[ACO04] Alberto Alvarez, Andrea Caiti, and Reiner Onken. Evolutionary path planning for autonomous underwater vehicles in a variable ocean. IEEE Journal of Oceanic Engineering, 29(2):418-429, 2004.

[AH06] Kjetil Bergh Anonsen and Oddvar Hallingstad. Terrain aided underwater navigation using point mass and particle filters. In Proceedings of the IEEE/ION Position Location and Navigation Symposium, 2006.

[AH07] Kjetil Bergh Anonsen and Oddvar Hallingstad. Sigma point kalman filter for underwater terrain-based navigation. In Proceedings of the IFAC conference on control applications in marine systems, pages 106-110, 2007.

[Bec15] Aaron T. Becker. Markov decision process grid world. matlab central file exchange. measurements, 2015.

[BFD ${ }^{+}$17] Fabian Blöchliger, Marius Fehr, Marcin Dymczyk, Thomas Schneider, and Roland Siegwart. Topomap: Topological mapping and navigation based on visual slam maps. arXiv preprint arXiv:1709.05533, 2017.

[BFH97] Wolfram Burgard, Dieter Fox, and Daniel Hennig. Fast grid-based position tracking for mobile robots. In Proceedings of the Annual Conference on Artificial Intelligence, pages 289-300. Springer, 1997.

[BHF98] R Bachmayer, S Humphris, and D Fornari. Oceanographic research using remotely operated underwater robotic vehicles: Exploration. Marine Technology Society Journal, Jan 1998.

[BKS10] Jonathan Binney, Andreas Krause, and Gaurav S Sukhatme. Informative path planning for an autonomous underwater vehicle. In Proceedings of the IEEE International Conference on Robotics and Automation (ICRA), pages 4791-4796, 2010.

[BKS13] Jonathan Binney, Andreas Krause, and Gaurav S Sukhatme. Optimizing waypoints for monitoring spatiotemporal phenomena. International Journal of Robotics Research, 32(8):873-888, 2013. 
[BL94] J.G. Bellingham and J.J. Leonard. Task configuration with layered control. In IARP 2nd Workshop on Mobile Robots for Subsea Environments, May 1994.

[BL02] P Bhatta and N E Leonard. Stabilization and coordination of underwater gliders. In Proc. 41st IEEE Conference on Decision and Control, volume 2, pages 2081-2086, 2002.

[BLF09] Alexander Bahr, John J Leonard, and Maurice F Fallon. Cooperative localization for autonomous underwater vehicles. The International Journal of Robotics Research, 28(6):714-728, 2009.

[BOAS15] Michael Burri, Helen Oleynikova, Markus W Achtelik, and Roland Siegwart. Real-time visual-inertial mapping, re-localization and planning onboard mavs in unknown environments. In Intelligent Robots and Systems (IROS), 2015 IEEE/RSJ International Conference on, pages 1872-1878. IEEE, 2015.

[BSF11] Matthew Brown, Sabine Süsstrunk, and Pascal Fua. Spatio-chromatic decorrelation by shift-invariant filtering. In Proceedings of the IEEE Computer Society Conference on Computer Vision and Pattern Recognition Workshops, pages 27-34, 2011.

[BSR07] L. Bird, A. Sherman, and J. P. Ryan. Development of an active, large volume, discrete seawater sampler for autonomous underwater vehicles. In Proc Oceans MTS/IEEE Conference, Vancouver, Canada, 2007.

[BW16] Laughlin DL Barker and Louis L Whitcomb. A preliminary survey of underwater robotic vehicle design and navigation for under-ice operations. In Intelligent Robots and Systems (IROS), 2016 IEEE/RSJ International Conference on, pages 2028-2035. IEEE, 2016.

[BZ05] J. G. Bellingham and Y. Zhang. Observing processes that vary in time and space with heterogeneous mobile networks. In International Workshop on Underwater Robotics, pages 9-16, Genoa, Italy, 2005.

[Cal10] E. Callaway. Chlorophyll. in school of chemistry., 2010.

[CD14] Tianfeng Chai and Roland R Draxler. Root mean square error (rmse) or mean absolute error (mae)?-arguments against avoiding rmse in the literature. Geoscientific model development, 7(3):1247-1250, 2014. 
[CKMB04] Elizabeth L. Creed, John Kerfoot, Chhaya Mudgal, and Herve' Barrier. Transition of slocum electric gliders to a sustained operational system. In OCEANS '04. MTTS/IEEE TECHNO-OCEAN '04, volume 2, pages 828833, November 2004.

$\left[\mathrm{CMG}^{+}\right.$02a] Elizabeth L Creed, Chhaya Mudgal, Scott Glenn, Oscar Schofield, Clayton Jones, and Douglas C Webb. Using a Fleet of Slocum Battery Gliders in a Regional Scale Coastal Ocean Observatory. In Oceans 'O2 MTS/IEEE, 2002.

$\left[\mathrm{CMG}^{+}\right.$02b] Elizabeth L. Creed, Chhaya Mudgal, Scott Glenn, Oscar Schofield, Clayton Jones, and Douglas C. Webb. Using a fleet of slocum battery gliders in a regional scale coastal ocean observatory. In Oceans 'O2 MTS/IEEE, 2002.

$\left[\mathrm{CMN}^{+}\right.$92] Kevin P Carroll, Stephen R McClaran, Eric L Nelson, David M Barnett, Donald K Friesen, and Glen N William. Auv path planning: an $\mathrm{A}^{*}$ approach to path planning with consideration of variable vehicle speeds and multiple, overlapping, time-dependent exclusion zones. In Proceedings of the Symposium on Autonomous Underwater Vehicle Technology (AUV), pages 79-84, 1992.

[CMR10] A. Comport, E. Malis, and P. Rives. Real-time Quadrifocal Visual Odometry. International Journal of Robotics Research, Special issue on Robot Vision, 29(2 - 3):245 - 266, 2010.

[CO81] Andrew David Cliff and J Keith Ord. Spatial processes: models \& applications. Taylor \& Francis, 1981.

[CZ08] Guangqing Chi and Jun Zhu. Spatial regression models for demographic analysis. Population Research and Policy Review, 27(1):17-42, 2008.

[CZMF16] Yiming Chen, Dongfang Zheng, Paul A Miller, and Jay A Farrell. Underwater inertial navigation with long baseline transceivers: a near-real-time approach. IEEE Transactions on Control Systems Technology, 24(1):240$251,2016$.

[DDCW98] Kris Demuynck, Jacques Duchateau, Dirk Van Compernolle, and Patrick Wambacq. Improved feature decorrelation for HMM-based speech recognition. In Proceedings of the Fifth International Conference on Spoken Language Processing, 1998. 
[Die03] F. Dieter. Adapting the sample size in particle filters through kld-sampling. The Int. J. of Robotics Research, 22(12):985-1003, 2003.

[DMM $\left.{ }^{+} 11\right]$ J Das, T Maughan, M McCann, M Godin, T O’Reilly, M Messie, F Bahr, K Gomes, F Py, J Bellingham, G Sukhatme, and K Rajan. Towards mixedinitiative, multi-robot field experiments: Design, deployment, and lessons learned. In Proceedings of the Intelligent Robots and Systems (IROS) Conference, San Francisco, 2011.

[Don12] Glenn T Donovan. Position error correction for an autonomous underwater vehicle inertial navigation system (ins) using a particle filter. IEEE Journal of Oceanic Engineering, 37(3):431-445, 2012.

[DOR ${ }^{+}$08] R. E. Davis, M.D. Ohman, D.L. Rudnick, J.T. Sherman, and B. Hodges. Glider surveillance of physics and biology in the southern california current system. Limnol. Oceanogr., 2008.

[DRA93] Murray D Dailey, Donald J Reish, and Jack W Anderson. Ecology of the Southern California Bight: a synthesis and interpretation. Univ of California Press, 1993.

[Dub98] Robin A Dubin. Spatial autocorrelation: a primer. Journal of housing economics, 7(4):304-327, 1998.

[Dyn18a] Boston Dynamics. Boston dynamics's atlas, 2018.

[Dyn18b] Boston Dynamics. Boston dynamics’s spotmini, 2018.

[ECR ${ }^{+}$06] GM Espindola, Gilberto Câmara, IA Reis, LS Bins, and AM Monteiro. Parameter selection for region-growing image segmentation algorithms using spatial autocorrelation. International Journal of Remote Sensing, 27(14):3035-3040, 2006.

[EKOL08a] L. H. Erickson, J. Knuth, J. M. O’Kane, and S. M. LaValle. Probabilistic localization with a blind robot. In Proc. of IEEE ICRA, pages 1821-1827, 2008.

[EKOL08b] Lawrence H Erickson, Joseph Knuth, Jason M O'Kane, and Steven M LaValle. Probabilistic localization with a blind robot. In Proceedings of the IEEE International Conference on Robotics and Automation, pages 1821-1827, 2008. 
[EL09] Jane Elith and JR Leathwick. Species distribution models: ecological explanation and prediction across space and time. Annual Review of Ecology, Evolution, and Systematics, 40:677-697, 2009.

[EL13] L. H. Erickson and S. M. LaValle. Toward the design and analysis of blind, bouncing robots. In Proc. of IEEE ICRA, pages 3233-3238, 2013.

$\left[\mathrm{EOL}^{+} 01\right]$ Charles C. Eriksen, T. James Osse, Russell D. Light, Timothy Wen, Thomas W. Lehman, Peter L. Sabin, John W. Ballard, and Andrew M. Chiodi. Seaglider: A long-range autonomous underwater vehicle for oceanographic research. IEEE Journal of Oceanic Engineering, 26(4):424-436, October 2001.

[EPH $\left.{ }^{+} 11\right] \quad J$ Elith, S J Phillips, T Hastie, M Dud'ik, Y E Chee, and C J Yates. A statistical explanation of maxent for ecologists. Diversity and Distributions, 17(1):43-57, 2011.

[ESRI18a] Inc. Environmental Systems Research Institute. How kriging works, 2018.

[ESRI18b] Inc. Environmental Systems Research Institute. How spline works, 2018.

[FBLS03] Edward Fiorelli, Pradeep Bhatta, Naomi Ehrich Leonard, and Igor Shulman. Adaptive sampling using feedback control of an autonomous underwater glider fleet. In Proceedings of the 13th International Symposium on Unmanned Untethered Submersible Technology (UUST), pages 1-16, 2003.

[FBT99] Dieter Fox, Wolfram Burgard, and Sebastian Thrun. Markov localization for mobile robots in dynamic environments. J. of Artificial Intelligence Research, 11:391-427, 1999.

[FDF02] E. Frazzoli, M.A. Daleh, and E. Feron. Real-time motion planning for agile autonomous vehicles. AIAA Journal of Guidance, Control and Dynamics, 25(1):116-129, 2002.

[FE14] Inc Fondriest Environmental. Algae, phytoplankton and chlorophyll. fundamentals of environmental measurements, 2014.

[FLCS12] C. Fookes, F. Lin, V. Chandran, and S. Sridharan. Evaluation of image resolution and super-resolution on face recognition performance. Journal of Visual Communication and Image Representation, 23(1):75 - 93, 2012. 
[Fox03] Dieter Fox. Adapting the sample size in particle filters through KLDsampling. International Journal of Robotics Research, 22(12):985-1003, 2003.

[FPL05] Jay A Farrell, Shuo Pang, and Wei Li. Chemical plume tracing via an autonomous underwater vehicle. IEEE Journal of Oceanic Engineering, 30(2):428-442, 2005.

[Fra82] Richard Franke. Smooth interpolation of scattered data by local thin plate splines. Computers \& Mathematics with Applications, 8(4):273-281, 1982.

[FZ03] C. Fruh and A. Zakhor. Constructing 3D city models by merging aerial and ground views. IEEE Computer Graphics and Applications, 23(6):52 $-61,2003$.

[GA10] Arthur Getis and Jared Aldstadt. Constructing the spatial weights matrix using a local statistic. In Perspectives on spatial data analysis, pages 147163. Springer, 2010.

[GAO05] Bartolome Garau, Alberto Alvarez, and Gabriel Oliver. Path planning of autonomous underwater vehicles in current fields with complex spatial variability: an A* approach. In Proceedings of the IEEE International Conference on Robotics and Automation (ICRA), pages 194-198, 2005.

[GB78] Arthur Getis and Barry N Boots. Models of spatial processes: an approach to the study of point, line and area patterns, volume 198. Cambridge University Press Cambridge, 1978.

[GBDR96] Anthony C Gatrell, Trevor C Bailey, Peter J Diggle, and Barry S Rowlingson. Spatial point pattern analysis and its application in geographical epidemiology. Transactions of the Institute of British geographers, pages 256-274, 1996.

[GCE $\left.{ }^{+} 13\right]$ K Gomes, D Cline, D Edgington, M Godin, T Maughan, M McCann, T O'Reilly, F Bahr, F Chavez, M Messi, J Das, and K Rajan. \{ODSS\}: A Decision Support System for Ocean Exploration. In Workshop on DataDriven Decision Guidance and Support Systems (DGSS) at the 29th IEEE International Conference on Data Engineering, Brisbane, Australia, 2013.

$\left[\mathrm{GGB}^{+}\right.$02] Fredrik Gustafsson, Fredrik Gunnarsson, Niclas Bergman, Urban Forssell, Jonas Jansson, Rickard Karlsson, and P-J Nordlund. Particle filters for po- 
sitioning, navigation, and tracking. Signal Processing, IEEE Transactions on, 50(2):425-437, 2002.

$\left[\mathrm{GGM}^{+}\right.$12] Jeremy Gottlieb, Rishi Graham, Thom Maughan, Fr??d??ric Py, Gabriel Elkaim, and Kanna Rajan. An experimental momentum-based front detection method for autonomous underwater vehicles. In Proceedings - IEEE International Conference on Robotics and Automation, pages 5322-5327, 2012.

[GJFB07] Gwynn Griffiths, Clayton Jones, James Ferguson, and Neil Bose. Undersea gliders. Feeding and Healing Humans, 2(2):64-75, 2007.

[Gol80a] Joe P Golden. Terrain contour matching (tercom): a cruise missile guidance aid. In 24th Annual Technical Symposium, pages 10-18. International Society for Optics and Photonics, 1980.

[Gol80b] Joe P Golden. Terrain contour matching (TERCOM): a cruise missile guidance aid. In Proceedings of the Image Processing for Missile Guidance, volume 238, pages 10-19, 1980 .

[Gri13] Daniel A Griffith. Spatial autocorrelation and spatial filtering: gaining understanding through theory and scientific visualization. Springer Science \& Business Media, 2013.

[Gro13] Paul D Groves. Principles of GNSS, inertial, and multisensor integrated navigation systems. Artech house, 2013.

[Haw18] Precision Hawk. Precision hawk’s dji matrice 210, 2018.

[HBH08] Oyvind Hegrenas, Einar Berglund, and Oddvar Hallingstad. Model-aided inertial navigation for underwater vehicles. In Robotics and Automation, 2008. ICRA 2008. IEEE International Conference on, pages 1069-1076. IEEE, 2008.

$\left[\mathrm{HHL}^{+}{ }^{14}\right.$ Lionel Heng, Dominik Honegger, Gim Hee Lee, Lorenz Meier, Petri Tanskanen, Friedrich Fraundorfer, and Marc Pollefeys. Autonomous visual mapping and exploration with a micro aerial vehicle. Journal of Field Robotics, 31(4):654-675, 2014.

[HMH07] Per Espen Hagen, Oivind Midtgaard, and Oistein Hasvold. Making auvs truly autonomous. In OCEANS 2007, pages 1-4. IEEE, 2007. 
[HMR12] Bharath Hariharan, Jitendra Malik, and Deva Ramanan. Discriminative decorrelation for clustering and classification. In Proceedings of the European Conference on Computer Vision, pages 459-472. Springer, 2012.

[HOBR14] Hoehun Ha, James R. Olson, Ling Bian, and Peter A. Rogerson. Analysis of heavy metal sources in soil using kriging interpolation on principal components. Environmental Science \& Technology, 48(9):4999-5007, 2014. PMID: 24693925.

[HTH15] Hadi Hajieghrary, Alex Fabregat Tomás, and M Ani Hsieh. An information theoretic source seeking strategy for plume tracking in $3 \mathrm{~d}$ turbulent fields. In Proceedings of the IEEE International Symposium on Safety, Security, and Rescue Robotics (SSRR), pages 1-8, 2015.

[HW16] Zachary J Harris and Louis L Whitcomb. Preliminary study of cooperative navigation of underwater vehicles without a dvl utilizing range and range-rate observations. In Robotics and Automation (ICRA), 2016 IEEE International Conference on, pages 2618-2624. IEEE, 2016.

[Ima18] Malin Space Science Systems (MSSS) Exploration Through Imaging. Curiosity rover takes selfie on mars, 2018.

[Inca] YSI Inc. / Xylem Inc. Dissolved oxygen measurement in water.

[Incb] YSI Inc. / Xylem Inc. Ecomapper auv - generate high-resolution maps of water quality, water currents, bathymetry, and sonar imagery.

[Incc] YSI Inc. / Xylem Inc. Temperature.

[Incd] YSI Inc. / Xylem Inc. Turbidity units, tss, water clarity, suspended particles measurement, turbidity in water.

[Inc14a] Patrick Higgins YSI Inc. / Xylem Inc. The ultimate ph primer - are you familiar with hydrogen?, 2014.

[Inc14b] Patrick Higgins (YSI Incorporated.). The basics of chlorophyll measurement. in ysi environmental tech note., 2014.

[Inc18] Alphabet Inc. Waymo: Google's self-driving car, 2018. 
[JCG ${ }^{+}$05a] Clayton Jones, Elizabeth L Creed, Scott Glenn, John Kerfoot, Josh Kohut, Chhaya Mudgal, and Oscar Schofield. Slocum Gliders - A Component of Operational Oceanography. In Autonomous Undersea Systems Institute Symposium Proceedings, 2005.

[JCG ${ }^{+}$05b] Clayton Jones, Elizabeth L. Creed, Scott Glenn, John Kerfoot, Josh Kohut, Chhaya Mudgal, and Oscar Schofield. Slocum gliders - a component of operational oceanography. In Autonomous Undersea Systems Institute Symposium Proceedings, 2005.

[JLV99a] L. Jetto, S. Longhi, and G. Venturini. Development and experimental validation of an adaptive extended kalman filter for the localization of mobile robots. IEEE Trans. on Robotics and Automation, 15(2):219-229, 1999.

[JLV99b] Leopoldo Jetto, Sauro Longhi, and Giuseppe Venturini. Development and experimental validation of an adaptive extended Kalman filter for the localization of mobile robots. IEEE Transactions on Robotics and Automation, 15(2):219-229, 1999.

[JN08] K. S. Johnson and J. A. Needoba. Mapping the spatial variability of plankton metabolism using nitrate and oxygen sensors on an autonomous underwater vehicle. Limnology and Oceanography, 53:22372250, 2008.

[JND02] B H Jones, M A Noble, and T D Dickey. Hydrographic and particle distributions over the Palos Verdes Continental Shelf: spatial, seasonal and daily variability. Continental Shelf Research, 22(6-7):945-965, 2002.

[JSPG09] Antonio R Jimenez, Fernando Seco, Carlos Prieto, and Jorge Guevara. A comparison of pedestrian dead-reckoning algorithms using a low-cost mems imu. In Intelligent Signal Processing, 2009. WISP 2009. IEEE International Symposium on, pages 37-42. IEEE, 2009.

[KEW06] James C Kinsey, Ryan M Eustice, and Louis L Whitcomb. A survey of underwater vehicle navigation: Recent advances and new challenges. In IFAC Conference of Manoeuvering and Control of Marine Craft, 2006.

[KG07] Andreas Krause and Carlos Guestrin. Near-optimal observation selection using submodular functions. In Proc. of 22nd Conference on Artificial Intelligence, 2007.

[KGGK06] A. Krause, C. Guestrin, A. Gupta, and J. Kleinberg. Near-optimal sensor placements: maximizing information while minimizing communication 
cost. In Information Processing in Sensor Networks, 2006. IPSN 2006. The Fifth International Conference on, pages 2-10, 2006.

[KHS15] Dhanushka Kularatne, M Ani Hsieh, and Ryan N. Smith. Zig-Zag Wanderer: Towards Adaptive Tracking of Time-Varying Coherent Structures in the Ocean. In IEEE International Conference on Robotics and Automation, Seattle, WA, May 2015.

[KIJT17] Shwesin Koko, Kim Irvine, Ranjna Jindal, and Romanee Thongdara. Spatial and temporal variations of dissolved oxygen in cha-am municipality wastewater treatment ponds using gis kriging interpolation. Journal of Water Management Modeling, 2017.

[KLS18] Agnan Kessy, Alex Lewin, and Korbinian Strimmer. Optimal whitening and decorrelation. The American Statistician, pages 1-6, 2018.

[KMGG08] Andreas Krause, H. Brendan McMahan, Carlos Guestrin, and Anupam Gupta. Robust submodular observation selection. Journal of Machine Learning Research, 9:2761-2801, December 2008.

[KP09] Michael Kearney and Warren Porter. Mechanistic niche modelling: Combining physiological and spatial data to predict species' ranges. Ecology Letters, 12(4):334-350, 2009.

[KRW09] Choo Leng Koh, Soydan Redif, and Stephan Weiss. Broadband GSC beamformer with spatial and temporal decorrelation. In Proceedings of the 17th European Conference on Signal Processing, pages 889-893, 2009.

[KSBB07] Dov Kruger, Rustam Stolkin, Aaron Blum, and Joseph Briganti. Optimal auv path planning for extended missions in complex, fast-flowing estuarine environments. In Proceedings of the IEEE International Conference on Robotics and Automation (ICRA), pages 4265-4270, 2007.

[KSG08] Andreas Krause, Ajit Singh, and Carlos Guestrin. Near-Optimal sensor placements in gaussian processes: Theory, efficient algorithms and empirical studies. J. Mach. Learn. Res., 9:235-284, 2008.

[KTK ${ }^{+}$02] Victor S Kennedy, Robert R Twilley, Joan A Kleypas, James H Cowan Jr, and Steven R Hare. Coastal and marine ecosystems \& global climate change. Technical report, Pew Center on Global Climate Change, 2002. 
[L $\mathrm{L}^{+}$12] Steven M LaValle et al. Sensing and filtering: A fresh perspective based on preimages and information spaces. Foundations and Trends in Robotics, 1(4):253-372, 2012.

[Lag10] Julien Lagadec. Terrain Based Navigation using a Particle Filter for Long range glider missions - Feasibility study and simulations. Master's thesis, 2010.

[LaV06] Steven M LaValle. Planning Algorithms. Cambridge University Press, Cambridge, U.K., 2006. Available at http://planning.cs.uiuc.edu.

[LB16] John J Leonard and Alexander Bahr. Autonomous underwater vehicle navigation. In Springer Handbook of Ocean Engineering, pages 341-358. Springer, 2016.

[LDK09] Kian Hsiang Low, John Dolan, and Pradeep Khosla. Information-theoretic approach to efficient adaptive path planning for mobile robotic environmental sensing. In Proceedings of the 19th International Conference on Automated Planning and Scheduling (ICAPS-09), September 2009.

[LDW91a] J. J. Leonard and H. F. Durrant-Whyte. Mobile robot localization by tracking geometric beacons. IEEE Trans. on Robotics and Automation, 7(3):376-382, 1991.

[LDW91b] John J Leonard and Hugh F Durrant-Whyte. Mobile robot localization by tracking geometric beacons. IEEE Transactions on Robotics and Automation, 7(3):376-382, 1991.

[Leg93] Pierre Legendre. Spatial autocorrelation: trouble or new paradigm? Ecology, 74(6):1659-1673, 1993.

[LF89] Pierre Legendre and Marie Josée Fortin. Spatial pattern and ecological analysis. Vegetatio, 80(2):107-138, 1989.

[LG01] Naomi Ehrich Leonard and Joshua G. Graver. Model-based feedback control of autonomous underwater gliders. IEEE Journal of Oceanic Engineering, Special Issue on Autonomous Ocean-Sampling Networks, 26(4):633-645, October 2001.

[LLL $\left.{ }^{+} 15\right] \quad$ Qiang Lu, Qi Shi Luo, Hui Li, Yong Di Liu, Ji Dong Gu, and Kuang Fei Lin. Characterization of chlorinated aliphatic hydrocarbons and en- 
vironmental variables in a shallow groundwater in shanghai using kriging interpolation and multifactorial analysis. PLOS ONE, 10(11):1-13, 11 2015.

[LLRX06] Andrew Lim, Jing Lin, Brian Rodrigues, and Fei Xiao. Ant colony optimization with hill climbing for the bandwidth minimization problem. Applied Soft Computing, 6(2):180-188, 2006.

$\left[\mathrm{LPD}^{+} 10\right] \quad$ Naomi E Leonard, Derek A Paley, Russ E Davis, David M Fratantoni, Francois Lekien, and Fumin Zhang. Coordinated control of an underwater glider fleet in an adaptive ocean sampling field experiment in monterey bay. Journal of Field Robotics, 27(6):718-740, 2010.

[LPL ${ }^{+}$07] N E Leonard, D A Paley, F Lekien, R Sepulchre, D M Fratantoni, and R E Davis. Collective motion, sensor networks, and ocean sampling. Proceedings of the IEEE, 95(1):48-74, 2007.

[LR06] Naomi E Leonard and Steve Ramp. Adaptive sampling and prediction, 2006.

[LSF07] C. Liu, H.-Y. Shum, and W. Freeman. Face Hallucination: Theory and Practice. Internaltional Journal of Computer Vision, 75(1):115 - 134, oct 2007.

[MCSed] Yurly Mileyko, Monique Chyba, and Ryan N. Smith. Energy-efficient control strategies for updating an augmented terrain-based navigation map for autonomous underwater navigation. In Proceedings of the IEEE Conference on Control Technology and Applications, Hawaii, August 2017, submitted.

$\left[\mathrm{MDM}^{+}{ }^{12}\right]$ Thom Maughan, Jnaneshwar Das, Mike McCann, Mike Godin, Fred Bahr, Kevin Gomes, Tom OReilly, Frederic Py, Monique Messie, John Ryan, Francisco Chavez, Jim Bellingham, Maria Fox, and Kanna Rajan. An oceanographic decision support system for scientific field experiments. In MTS/IEEE Oceans, 2012.

[MFC77] Sylvie Rimbert Marie France Cicri, Bernard Marchand. Introduction l'analyse de l'espace. Number 978-2225470363. Masson, 1977.

[MKE15] L. Medagoda, J. C. Kinsey, and M. Eilders. Autonomous underwater vehicle localization in a spatiotemporally varying water current field. In 2015 
IEEE International Conference on Robotics and Automation (ICRA), pages 565-572, May 2015.

[MLS16] Kai-Chieh Ma, Lantao Liu, and Gaurav S Sukhatme. An informationdriven and disturbance-aware planning method for long-term ocean monitoring. In Proceedings of IEEE/RSJ International Conference on Intelligent Robots and Systems (IROS), pages 2102-2108, 2016.

[MLS17] Kai-Chieh Ma, Lantao Liu, and Gaurav S Sukhatme. Informative planning and online learning with sparse gaussian processes. In Proceedings of the IEEE International Conference on Robotics and Automation (ICRA), pages 4292-4298, 2017.

[MLW ${ }^{+}$16] Travis Miles, Sang Hoon Lee, Anna Wåhlin, Ho Kyung Ha, Tae Wan Kim, Karen M Assmann, and Oscar Schofield. Glider observations of the dotson ice shelf outflow. Deep Sea Research Part II: Topical Studies in Oceanography, 123:16-29, 2016.

[MM88] L Mitáš and H Mitášová. General variational approach to the interpolation problem. Computers \& Mathematics with Applications, 16(12):983-992, 1988.

[MM15] Andrew Mccall and John K Mckay. Experimental verification of ecological niche modeling in a heterogeneous environment EXPERIMENTAL VERIFICATION OF ECOLOGICAL NICHE MODELING IN. 9658(August):2433-2439, 2015.

[MM17] José Melo and Aníbal Matos. Survey on advances on terrain based navigation for autonomous underwater vehicles. Ocean Engineering, 139:250264, 2017.

[MPR08a] C McGann, F Py, and K Rajan. On efficient deliberation and execution. In Submitted to ICAPS, Intnl. Conf. on Automated Planning and Scheduling, 2008.

[MPR ${ }^{+}$08b] C McGann, F Py, K Rajan, J P Ryan, and R Henthorn. Adaptive control for autonomous underwater vehicles. In $A A A I, 2008$.

[MPR ${ }^{+}$08c] C McGann, F Py, K Rajan, J P Ryan, H Thomas, R Henthorn, and $\mathrm{R}$ McEwen. Preliminary results for model-based adaptive control of an autonomous underwater vehicle. In Intnl. Symp. on Experimental Robotics (ISER), 2008. 
[MRR ${ }^{+}$15] Ross Marchant, Dean Reading, James Ridd, Sean Campbell, and Peter Ridd. A drifter for measuring water turbidity in rivers and coastal oceans. Marine Pollution Bulletin, 91(1):102 - 106, 2015.

$\left[\mathrm{MSM}^{+} 12\right]$ Nathan Michael, Shaojie Shen, Kartik Mohta, Yash Mulgaonkar, Vijay Kumar, Keiji Nagatani, Yoshito Okada, Seiga Kiribayashi, Kazuki Otake, Kazuya Yoshida, et al. Collaborative mapping of an earthquakedamaged building via ground and aerial robots. Journal of Field Robotics, 29(5):832-841, 2012.

[MTMT09] Xavier Morin, Wilfried Thuiller, Xavier Morin1', and Wilfried Thuiller3. Comparing Niche-and Process-Based Models to Reduce Prediction Uncertainty in Species Range Shifts under Climate Change. Source: Ecology Ecology, 90(905):1301-1313, 2009.

[NAS18] NASA. The mars science laboratory's curiosity rover, 2018.

[NDH14] Woonhyun Nam, Piotr Dollár, and Joon Hee Han. Local decorrelation for improved pedestrian detection. In Proceedings of Advances in Neural Information Processing Systems, pages 424-432, 2014.

[OAM16] LLC. OMRON Adept MobileRobots. Aqua2 autonomous underwater vehicle, 2016.

[oEB 18] The Editors of Encyclopaedia Britannica. Temperature - physics, 2018.

[OL05] J. M. O'Kane and S. M. LaValle. Almost-sensorless localization. In Proc. of ICRA, pages 3764-3769, 2005.

[OL07a] J. M. O'Kane and S. M. LaValle. Localization with limited sensing. IEEE Trans. on Robotics, 23(4):704-716, 2007.

[OL07b] Jason M O'Kane and Steven M LaValle. Localization with limited sensing. IEEE Transactions on Robotics, 23(4):704, 2007.

[OLCJ14] Ruofei Ouyang, Kian Hsiang Low, Jie Chen, and Patrick Jaillet. Multirobot active sensing of non-stationary gaussian process-based environmental phenomena. In Proceedings of International Conference on Autonomous Agents and Multi-Agent Systems (AAMAS), pages 573-580, 2014. 
[oTTJPL18a] NASA California Institute of Technology's The Jet Propulsion Laboratory. Curiosity rover takes selfie on mars, 2018.

[oTTJPL18b] NASA California Institute of Technology's The Jet Propulsion Laboratory. Nasa's mars helicopter, 2018.

[Pai] John Paisley. A tutorial on the dirichlet process for engineers technical report.

[PBHS13] Arvind A. Pereira, Jonathan Binney, Geoffrey A. Hollinger, and Gaurav S. Sukhatme. Risk-aware Path Planning for Autonomous Underwater Vehicles using Predictive Ocean Models. Journal of Field Robotics, 30(5):741762, September 2013.

[Pet06] AT Peterson. Uses and requirements of ecological niche models and related distributional models. Biodiversity Informatics, 3:59-72, 2006.

[PEWF08] S. Prince, J. Elder, J. Warrell, and F. Felisberti. Tied factor analysis for face recognition across large pose differences. IEEE Transactions on Pattern Analysis and Machine Intelligence, 30(6):970 - 984, 2008.

[Pie77] Evelyn Chris Pielou. Mathematical ecology. Number 574.50151. Wiley, 1977.

[PPP ${ }^{+}$07] Clment Petres, Yan Pailhas, Pedro Patron, Yvan Petillot, Jonathan Evans, and David Lane. Path planning for autonomous underwater vehicles. IEEE Transactions on Robotics, 23(2):331-341, 2007.

[PT02] Andrada I Pacheco and Timothy J Tyrrell. Testing spatial patterns and growth spillover effects in clusters of cities. Journal of Geographical Systems, 4(3):275-285, 2002.

[PZL08a] D. Paley, F. Zhang, and N.E. Leonard. Cooperative control for ocean sampling: The glider coordinated control system. IEEE Transactions on Control Systems Technology, 16(4):735-744, 2008.

[PZL08b] D A Paley, F Zhang, and N E Leonard. Cooperative Control for Ocean Sampling: The Glider Coordinated Control System. IEEE Transactions on Control Systems Technology, 16(4):735-744, July 2008. 
[PZL08c] Derek A Paley, Fumin Zhang, and Naomi Ehrich Leonard. Cooperative control for ocean sampling: The glider coordinated control system. IEEE Transactions on Control Systems Technology, 16(4):735-744, 2008.

[Raj] Kanna Rajan. The role of deliberation ( and ai ) in marine robots.

[RDE $\left.{ }^{+}\right] \quad$ Daniel L Rudnick, Russ E Davis, Charles C Eriksen, David M Fratantoni, and Mary Jane Perry. Underwater Gliders for Ocean Research. Marine Technology Society Journal, 38(2):73-84.

[REDF08] Naomi E. Leonard Russ E. Davis and David M. Fratantoni. Routing strategies for underwater gliders. Deep Sea Research Part II: Topical Studies in Oceanography, September 2008. Article in press, doi:10.1016/j.dsr2.2008.08.005.

$\left[\mathrm{REH}^{+} 11\right] \quad$ L. M. Robinson, J. Elith, A. J. Hobday, R. G. Pearson, B. E. Kendall, H. P. Possingham, and A. J. Richardson. Pushing the limits in marine species distribution modelling: Lessons from the land present challenges and opportunities. Global Ecology and Biogeography, 20(6):789-802, 2011.

[RFA ${ }^{+}$17a] Gregory Murad Reis, Michael Fitzpatrick, Jacob Anderson, Leonardo Bobadilla, and Ryan N Smith. Augmented terrain-based navigation to enable persistent autonomy for underwater vehicles. In Robotic Computing (IRC), IEEE International Conference on, pages 292-298. IEEE, 2017.

$\left[\mathrm{RFA}^{+}\right.$17b] Gregory Murad Reis, Michael Fitzpatrick, Jacob Anderson, Leonardo Bobadilla, and Ryan N Smith. Increasing persistent navigation capabilities for underwater vehicles with augmented terrain-based navigation. In MTS/IEEE Oceans, 2017.

$\left[\mathrm{RFB}^{+}\right.$17a] Gregory Murad Reis, Michael Fitzpatrick, Leonardo Bobadilla, Jacob Anderson, and Ryan N. Smith. Augmented Terrain-Based Navigation to Enable Persistent Autonomy for Underwater Vehicles. In IEEE International Conference on Robotic Computing, pages 1 - 7, Taichung, Taiwan, April 2017.

$\left[\mathrm{RFB}^{+}\right.$17b] Gregory Murad Reis, Michael Fitzpatrick, Leonardo Bobadilla, Jacob Anderson, and Ryan N. Smith. Augmented terrain-based navigation to enable persistent autonomy for underwater vehicles. In Proceedings of the IEEE International Conference on Robotic Computing, pages 292-298, 2017. 
[RH13] B L Reed and F S Hover. Tracking ocean fronts with multiple vehicles and mixed communication losses. In Intelligent Robots and Systems (IROS), 2013 IEEE/RSJ International Conference on, pages 3374-3381, 112013.

[RH14] Brooks Reed and Franz Hover. Oceanographic pursuit: Networked control of multiple vehicles tracking dynamic ocean features. Methods in Oceanography, pages 1-23, 52014.

[Rip81] Braian D Ripley. Spatial statistics. 1981. Hayward Wiley, New York, 1981.

[Rip87] BD Ripley. Spatial point pattern analysis in ecology. In Develoments in Numerical Ecology, pages 407-429. Springer, 1987.

[RM15] Robert A. Rohde and Richard A. Muller. Air pollution in china: Mapping of concentrations and sources. PLOS ONE, 10(8):1-14, 082015.

[Rob18] Clearpath Robotics. Heron unmanned surface vessel, 2018.

[RPB12] K Rajan, F Py, and J Barreiro. Towards deliberative control in marine robotics. In M Seto, editor, Autonomy in Marine Robots. 2012.

[RRTN08] David Ribas, Pere Ridao, Juan Domingo Tardós, and José Neira. Underwater slam in man-made structured environments. Journal of Field Robotics, 25(11-12):898-921, 2008.

$\left[\mathrm{SBT}^{+}\right.$17] Martin Saska, Tomas Baca, Justin Thomas, Jan Chudoba, Libor Preucil, Tomas Krajnik, Jan Faigl, Giuseppe Loianno, and Vijay Kumar. System for deployment of groups of unmanned micro aerial vehicles in gps-denied environments using onboard visual relative localization. Autonomous Robots, 41(4):919-944, Apr 2017.

[SCL ${ }^{+}$10a] Ryan N Smith, Yi Chao, Peggy P Li, David A Caron, Burton H Jones, and Gaurav S Sukhatme. Planning and implementing trajectories for autonomous underwater vehicles to track evolving ocean processes based on predictions from a regional ocean model. International Journal of Robotics Research, 29(12):1475-1497, 2010.

[SCL $\left.{ }^{+} 10 b\right]$ Ryan N Smith, Yi Chao, Peggy P Li, David A Caron, Burton H Jones, and Gaurav S Sukhatme. Planning and Implementing Trajectories for Autonomous Underwater Vehicles to Track Evolving Ocean Processes based 
on Predictions from a Regional Ocean Model. International Journal of Robotics Research, 29(12):1475-1497, October 2010.

[SDH $\left.{ }^{+} 10\right] \quad$ Ryan N Smith, Jnaneshwar Das, Hordur Heidarsson, Arvind Pereira, Ivona Cetinić, Lindsay Darjany, Marie-Ève Garneau, Meredith D Howard, Carl Oberg, Matthew Ragan, Astrid Schnetzer, Erica Seubert, Ellen C Smith, Beth A Stauffer, Gerardo Toro-Farmer, David A Caron, Burton H Jones, and Gaurav S Sukhatme. \{USC $\}$ CCINAPS $\}$ Builds Bridges: Observing and Monitoring the $\{\mathrm{S}\}$ outhern $\{\mathrm{C}\}$ alifornia $\{\mathrm{B}\}$ ight. IEEE Robotics and Automation Magazine, Special Issue on Marine Robotics Systems, 17(1):20-30, March 2010.

[SDOV01] Jeff Sherman, Russ E. Davis, W. B. Owens, and J. Valdes. The autonomous underwater glider "Spray". IEEE Journal of Oceanic Engineering, 26(4):437-446, October 2001.

[SDY ${ }^{+}$10] Ryan N. Smith, Jnaneshwar Das, Chao Yi, David A. Caron, Burton H. Jones, and Gaurav S. Sukhatme. Cooperative multi-AUV tracking of phytoplankton blooms based on ocean model predictions. In MTS/IEEE OCEANS 2010, pages 1 -10, Sydney, Australia, May 2010.

[SE12] T. Senlet and A. Elgammal. Satellite image based precise robot localization on sidewalks. In IEEE International Conference on Robotics and Automation (ICRA), pages 2647 - 2653, may 2012.

[SH14] Ryan N Smith and Van T Huynh. Controlling buoyancy-driven profiling floats for applications in ocean observation. IEEE Journal of Oceanic Engineering, 39(3):571-586, 2014.

[SHLC16] Kevin Smith, Shih-Chieh Hsiung, Christopher G. Lowe, and Christopher M. Clark. Stochastic Modeling and Control for Tracking the Periodic Movement of Marine Animals via AUVs. In IEEE/RSJ International Conference on Intelligent Robots and Systems., Daejeon, Korea, oct 2016.

$\left[\mathrm{SKA}^{+}\right.$07] O. Schofield, J. Kohut, D. Aragon, E.L. Creed, J.G. Graver, C. Haldman, J. Kerfoot, H. Roarty, C. Jones, D.C. Webb, and S. Glenn. Slocum gliders: Robust and ready. Journal of Field Robotics, 24(6):473-485, 2007.

[SKC $\left.{ }^{+} 10\right] \quad$ Ryan N Smith, Jonathan Kelly, Yi Chao, Burton H Jones, and Gaurav S Sukhatme. Towards improvement of autonomous glider navigation accuracy through the use of regional ocean models. In Proceedings of In- 
ternational Conference on Offshore Mechanics and Arctic Engineering (OMAE), pages 597-606, 2010.

[SKGK09] Amarjeet Singh, Andreas Krause, Carlos Guestrin, and William Kaiser. Efficient informative sensing using multiple robots. Journal of Artificial Intelligence Research, 34:707-755, 2009.

[SKS12a] R. N. Smith, J. Kelly, and G. S. Sukhatme. Towards improving mission execution for autonomous gliders with an ocean model and kalman filter. In 2012 IEEE International Conference on Robotics and Automation, pages 4870-4877, May 2012.

[SKS12b] Ryan N. Smith, Jonathan Kelly, and Gaurav S. Sukhatme. Towards improving mission execution for autonomous gliders with an ocean model and Kalman filter. In Proceedings - IEEE International Conference on Robotics and Automation, pages 4870-4877, 2012.

[SKS16] Andrew Stuntz, Jonathan Scott Kelly, and Ryan N. Smith. Enabling Persistent Autonomy for Underwater Gliders with Ocean Model Predictions and Terrain-Based Navigation. Frontiers in Robotics and AI, 3:23, apr 2016.

[SLS15] A. Stuntz, D. Liebel, and R. N. Smith. Enabling persistent autonomy for underwater gliders through terrain based navigation. In OCEANS 2015 Genova, pages 1-10, May 2015.

[SM14] Z. Song and K. Mohseni. Towards background flow based auv localization. In 53rd IEEE Conference on Decision and Control, pages 6945-6950, Dec 2014.

[SMS ${ }^{+}$07] A Schnetzer, P E Miller, R A Schaffner, B Stauffer, B H Jones, S B Weisberg, P M DiGiacomo, W M Berelson, and D A Caron. Blooms of Pseudonitzschia and Domoic Acid in the San Pedro Channela and Los Angeles harbor areas of the Southern California Bight, 2003-2004. Harmful Algae, 6(3):372-387, 2007.

[SN04] Roland Siegwart and Illah R. Nourbakhsh. Introduction to Autonomous Mobile Robots. Bradford Company, Scituate, MA, USA, 2004.

[Sob10] Jorge M. Soberón. Niche and area of distribution modeling: A population ecology perspective. Ecography, 33(1):159-167, 2010. 
[Spe97] B. R. Speer. Photosynthetic pigments. in glossary., 1997.

[ŚPMD17] Jan Śliwka, Roberto Petroccia, Andrea Munafò, and Vladimir Djapic. Experimental evaluation of net-lbl: An acoustic network-based navigation system. In OCEANS 2017-Aberdeen, pages 1-9. IEEE, 2017.

[SSS ${ }^{+}$11a] Ryan N Smith, Mac Schwager, Stephen L Smith, Burton H Jones, Daniela Rus, and Gaurav S Sukhatme. Persistent Ocean Monitoring with Underwater Gliders: Adapting Sampling Resolution. Journal of Field Robotics, 28(5):714-741, 2011.

[SSS ${ }^{+}$11b] Ryan N Smith, Mac Schwager, Stephen L Smith, Burton H Jones, Daniela Rus, and Gaurav S Sukhatme. Persistent ocean monitoring with underwater gliders: Adapting sampling resolution. Journal of Field Robotics, 28(5):714-741, 2011.

[ST04] Noah A Smith and Roy W Tromble. Sampling uniformly from the unit simplex. Johns Hopkins University, Tech. Rep, 29, 2004.

[Sto06] D. R B Stockwell. Improving ecological niche models by data mining large environmental datasets for surrogate models. Ecological Modelling, 192(1-2):188-196, 2006.

[STR ${ }^{+}$13] Korbinian Schmid, Teodor Tomic, Felix Ruess, Heiko Hirschmüller, and Michael Suppa. Stereo vision based indoor/outdoor navigation for flying robots. In Intelligent Robots and Systems (IROS), 2013 IEEE/RSJ International Conference on, pages 3955-3962. IEEE, 2013.

[SYB97a] H Singh, D Yoerger, and A Bradley. Issues in auv design and deployment for oceanographic research. In Proceedings of the 1997 IEEE International Conference on Robotics and Automation, volume 3, pages 18571862, 1997.

[SYB97b] H. Singh, D. Yoerger, and A. Bradley. Issues in auv design and deployment for oceanographic research. In Proceedings of the 1997 IEEE International Conference on Robotics and Automation, volume 3, pages 18571862, 1997. Invited paper.

[TBF05] Sebastian Thrun, Wolfram Burgard, and Dieter Fox. Probabilistic robotics. MIT press, 2005. 
[TBSI10] Pratap Tokekar, Deepak Bhadauria, Andrew Studenski, and Volkan Isler. A robotic system for monitoring carp in minnesota lakes. Journal of Field Robotics, 27(6):779-789, 2010.

[TBVHI13] Pratap Tokekar, Elliot Branson, Joshua Vander Hook, and Volkan Isler. Tracking aquatic invaders: Autonomous robots for monitoring invasive fish. IEEE Robotics \& Automation Magazine, 20(3):33-41, 2013.

[TFBD01] S. Thrun, D. Fox, W. Burgard, and F. Dellaert. Robust monte carlo localization for mobile robots. Artificial Intelligence, 128(1-2):99-141, 2001.

[TIF14] Pratap Tokekar, Volkan Isler, and Antonio Franchi. Multi-target visual tracking with aerial robots. In Proceedings of the IEEE/RSJ International Conference on Intelligent Robots and Systems, pages 3067-3072, 2014.

[TLCP09] Pui-Jen Tsai, Men-Lung Lin, Chien-Min Chu, and Cheng-Hwang Perng. Spatial autocorrelation analysis of health care hotspots in taiwan in 2006. BMC Public Health, 9(1):464, 2009.

[Tun83] Yeou-Koung Tung. Point rainfall estimation for a mountainous region. Journal of Hydraulic Engineering, 109(10):1386-1393, 1983.

$\left[\mathrm{UF}^{+} 85\right]$ Graham Upton, Bernard Fingleton, et al. Spatial data analysis by example. Volume 1: Point pattern and quantitative data. John Wiley \& Sons Ltd., 1985.

[UF89] Graham JG Upton and Bernard Fingleton. Categorical and directional data. John Wiley and Sons, 1989.

[URO ${ }^{+}$08] Ben Upcroft, M.F. Ridley, L. Ong, B. Douillard, T. Kaupp, S. Kumar, T.A. Bailey, Fabio Ramos, A. Makarenko, A. Brooks, S. Sukkarieh, and H F Durrant-Whyte. Multi-level state estimation in an outdoor decentralised sensor network. Springer Tracts in Advanced Robotics - Experimental Robotics, 39:355 - 365, 2008.

[WAM04] Thorsten Wiegand and Kirk A Moloney. Rings, circles, and null-models for point pattern analysis in ecology. Oikos, 104(2):209-229, 2004.

[Wat85] David F Watson. A refinement of inverse distance weighted interpolation. Geoprocessing, 2:315-327, 1985. 
[WBZB01] J. S. Willcox, J. G. Bellingham, Y. Zhang, and A. B. Baggeroer. Performance metrics of oceanographic surveys with autonomous underwater vehicles. IEEE Journal of Oceanic Engineering, 26(4):711-725, 2001.

[WD08] Jonas Witt and Matthew Dunbabin. Go with the flow: Optimal auv path planning in coastal environments. In Proceedings of the Australasian Conference on Robotics and Automation (ACRA), 2008.

[Wet01] Robert G Wetzel. Limnology: lake and river ecosystems. gulf professional publishing, 2001.

[WF04] Jonathan A Warrick and Derek A Fong. Dispersal scaling from the world's rivers. Geophysical Research Letters, 31(4), 2004.

[WM13] Thorsten Wiegand and Kirk A Moloney. Handbook of spatial point-pattern analysis in ecology. Chapman and Hall/CRC, 2013.

[WYSH99a] L.L. Whitcomb, D.R. Yoerger, H. Singh, and J. Howland. Advances in underwater robot vehicles for deep ocean exploration: Navigation, control, and survey operations. In Proceedings of the Ninth International Symposium of Robotics Research, Snowbird, UT, 1999.

[WYSH99b] Louis L Whitcomb, Dana R Yoerger, Hanumant Singh, and Jonathan Howland. Combined doppler/lbl based navigation of underwater vehicles. In Proceedings of the the 11th international symposium on unmanned untethered submersible technology, volume 9818. AUSI, 1999.

[WYSH09] L. L. Whitcomb, D. R. Yoerger, H. Singh, and J. Howland. Advances in underwater robot vehicles for deep ocean exploration: Navigation, control, and survey operations. In Robotics Research - The Ninth International Symposium, London, 2009. Springer-Verlag Publications.

[WYSM98] L.L. Whitcomb, D.R. Yoerger, H. Singh, and D. Mindell. Towards precision robotic maneuvering, survey, and manipulation in unstructured undersea environments. In Robotics Research - The Eighth International Symposium, pages 45-54, London, 1998. Springer-Verlag Publications.

[YS85] D.N. Yoerger and J.E. Slotine. Robust trajectory control of underwater vehicles. IEEE Journal of Oceanic Engineering, 10(4):462-470, 1985.

[ysi17] Ysi EcoMapper, 2017. https://www.ysi.com/ecomapper. 
[Yuh00] J Yuh. Design and control of autonomous underwater robots: A survey. Autonomous Robots, 8:7-24, 2000.

[ZB08] Y. Zhang and J. G. Bellingham. An efficient method of selecting ocean observing locations for capturing the leading modes and reconstructing the full field. Journal of Geophysical Research (Oceans), 113(C04005), 2008. doi: 10.1029/2007JC004327.

[ZBB01] Y. Zhang, A. B. Baggeroer, and J. G. Bellingham. Spectral-feature classification of oceanographic processes using an autonomous underwater vehicle. IEEE Journal of Oceanic Engineering, 26(4):726-741, 2001.

[ZBB05] Y. Zhang, A. B. Baggeroer, and J. G. Bellingham. The total variance of a periodogram-based spectral estimate of a stochastic process with spectral uncertainty and its application to classifier design. IEEE Transactions on Signal Processing, 53(12):4556-4567, 2005.

[ZBC09] Y. Zhang, J. G. Bellingham, and Yi Chao. Error analysis and sampling strategy design for using fixed or mobile platforms to estimate ocean flux. Journal of Atmospheric and Oceanic Technology, 2009. In review.

$\left[Z_{B R}^{+}\right.$13] Yanwu Zhang, J G Bellingham, J P Ryan, B Kieft, and M J Stanway. Twodimensional mapping and tracking of a coastal upwelling front by an autonomous underwater vehicle. In MTS/IEEE Oceans - San Diego, pages 1-4, September 2013.

[ZMRB09a] Y. Zhang, R. S. McEwen, J. P. Ryan, and J. G. Bellingham. An adaptive triggering method for capturing peak samples in a thin phytoplankton layer by an autonomous underwater vehicle. In MTS/IEEE Oceans 2009, pages 1-5, Biloxi, Mississippi, U.S.A., 2009.

[ZMRB09b] Y Zhang, R S McEwen, J P Ryan, and J G Bellingham. An adaptive triggering method for capturing peak samples in a thin phytoplankton layer by an autonomous underwater vehicle. In MTS/IEEE Oceans 2009, pages $1-5,2009$.

[ZRB ${ }^{+}$12] Y Zhang, J P Ryan, J G Bellingham, J Harvey, and R S McEwen. Autonomous Detection and Sampling of Water Types and Fronts in Coastal Upwelling System by an Autonomous Underwater Vehicle. Limnology and Oceanography Methods, 10:934-951, 2012. 
[ZS08] Bin Zhang and Gaurav S Sukhatme. Adaptive sampling with multiple mobile robots. In IEEE International Conference on Robotics and Automation (submitted), 2008.

[ZSC $\left.{ }^{+} 16\right]$ Tao Zhang, Hongfei Shi, Liping Chen, Yao Li, and Jinwu Tong. Auv positioning method based on tightly coupled sins/lbl for underwater acoustic multipath propagation. Sensors, 16(3):357, 2016.

[ZXX15] Tao Zhang, Xiaosu Xu, and Shengbao Xu. Method of establishing an underwater digital elevation terrain based on kriging interpolation. Measurement, 63(Supplement C):287 - 298, 2015. 
VITA

\section{GREGORY MURAD REIS}

February 12, 1990

2008-2012

2012-2014

2014

2016-2018

2014-2018
Born, Lavras, Minas Gerais, Brazil

B.S., Computer Science

Federal University of Lavras

Lavras, Minas Gerais, Brazil

M.S., Systems Engineering

Federal University of Lavras

Lavras, Minas Gerais, Brazil

Professor, Mathematics

Federal University of Lavras

Lavras, Minas Gerais, Brazil

Instructor, Robotics Workshop

Ultimate Software Academy

Miami, FL

Ph.D., Computer Science

Florida International University

Miami, FL

\section{PUBLICATIONS}

Reis, G. M.; Leon, H.; Alam, T.; Anderson, J.; Bobadilla, L.; Smith, R. N. "A Whiteningbased Tracking Algorithm for Autonomous Underwater Vehicles", Accepted in Marine Technology Society/IEEE Oceans, Kobe, Japan, 2018.

Alam, T.; Reis, G. M.; Bobadilla, L.; Smith, R. N. "A Data-Driven Planning with the Persistent Behavior Analysis of a Marine Environment", Accepted in Marine Technology Society/IEEE Oceans, Kobe, Japan, 2018 (TOP 20).

Alam, T.; Reis, G. M.; Bobadilla, L.; Smith, R. N. "A Data-Driven Deployment Approach for Persistent Monitoring in Aquatic Environments", Accepted in IEEE International Conference on Robotic Computing, Laguna Hills, CA, USA, pp. 147 - 154, 2018.

Reis, G. M.; Fitzpatrick, M.; Bobadilla, L.; Anderson, J.; Smith, R.N., 'Increasing Persistent Navigation Capabilities for Underwater Vehicles with Augmented Terrain-Based Navigation", in Marine Technology Society/IEEE Oceans, Aberdeen, Scotland, 2017 
(TOP 20).

Reis, G. M.; Fitzpatrick, M.; Bobadilla, L.; Anderson, J.; Smith, R.N., "Augmented Terrain-Based Navigation to Enable Persistent Autonomy for Underwater Vehicle", in IEEE International Conference on Robotic Computing, Taichung, Taiwan, pp. 1-7, 2017.

Mileyko, Y.; Reis, G. M.; Chyba, M.; Smith, R.N., "Energy-Efficient Control Strategies for Updating an Augmented Terrain-Based Navigation Map for Autonomous Underwater Navigation", in IEEE Conference on Control Technology and Applications, Kohala Coast, Hawaií, 2017.

Ponciano, P. F.; Yanagi Junior, T.; Alvarenga, T. A. C.; Reis, G. M.; Campos, A. T. "Behavior of Chicks Subjected to Heat Stress", in XLII Brazilian Conference of Agricultural Engineering, Fortaleza, 2013.

Schiassi, L; Junior, T. Y.; Reis, G. M.; Abreu, L. H. P.; Campos, A. T.; Castro, J. O. "Fuzzy model- ing applied in the evaluation of broiler performance", in Revista Brasileira de Engenharia Agricola e Ambiental, Campina Grande, PB, BR, February 2015.

Ferraz, P. F. P.; Junior, T. Y.; Alvarenga, T. A. C.; Reis, G. M., Campos, A. T.; "Behavior of Chicks Subjected to Thermal Challenge", in Engenharia Agricola, Jaboticabal, SP, BR, December 2014.

Schiassi, L.; Junior, T. Y.; Abreu, L. H. P.; Reis, G. M., Damasceno, F. A.; Silva, G. C. A.; Campos, A. T. "Laboratory Proposal for Studies on Poultry Environment", in International Journal of Engineering Research and Applications, September 2014.

Ferraz, P. F. P.; Junior, T. Y.; Julio, Y. F. H.; Castro, J. O.; Gates, R. S.; Reis, G. M.; Campos, A. T.,'Predicting Chick Body Mass by Artificial Intelligence-Based Models",in Brazilian Journal of Agricultural Research, v. 49. n. 7, p. 559 568, July 2014. 